\title{
Investigation of early assembly of OXPHOS complexes during mitochondrial translation
}

\author{
Dissertation \\ for the award of the degree "Doctor rerum naturalium" at the \\ Georg-August-University Göttingen \\ within the doctoral program "Molecular Biology of Cells" of the \\ Georg-August University School of Science (GAUSS)
}

Submitted by

Cong Wang

born in Henan, China

Göttingen, 2018 


\section{Members of the Thesis Committee}

Prof. Dr. Peter Rehling

(Supervisor and first referee)

Prof. Dr. Markus Bohnsack

(Second referee)

Prof. Dr. Henning Urlaub
Institute for Cellular Biochemistry

University Medical Center

Göttingen, Germany

Institute for Molecular Biology

University Medical Center

Göttingen, Germany

Bioanalytical Mass Spectrometry

Max Plack Institute for Biophysical Chemistry

Göttingen, Germany

\section{Additional Members of the Examination Board}

Prof. Dr. Holger Stark

Prof. Dr. Stefan Jakobs

Dr. Alexander Stein

Date of oral examination: 14.09.2018
Structural Dynamics

Max Plack Institute for Biophysical Chemistry

Göttingen, Germany

Mitochondrial Structure and Dynamics

Max Plack Institute for Biophysical Chemistry

Göttingen, Germany

Membrane Protein Biochemistry

Max Plack Institute for Biophysical Chemistry

Göttingen, Germany 


\section{Affidavit}

I hereby declare that my dissertation, entitled "Assessing early assembly intermediates of OXPHOS complexes during mitochondrial translation", has been written independently and with no other aids or sources than quoted.

Cong Wang

Göttingen, July 2018 


\section{Table of Contents}

Affidavit............................................................................ iii

Table of Contents ........................................................... iv

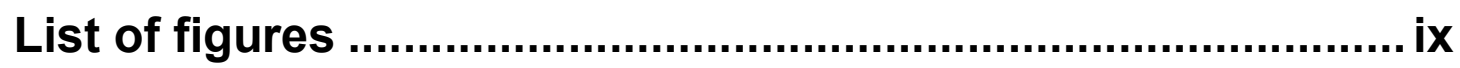

List of Tables ........................................................................ xii

List of abbreviations ........................................................... xiii

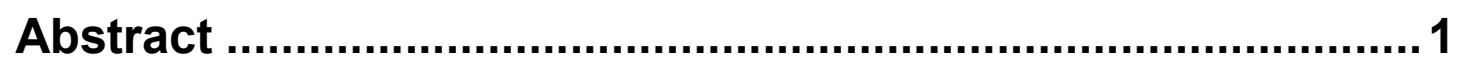

1 Introduction ....................................................................... 2

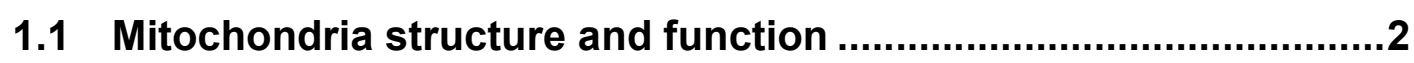

1.1.1 The Oxidative phosphorylation system......................................

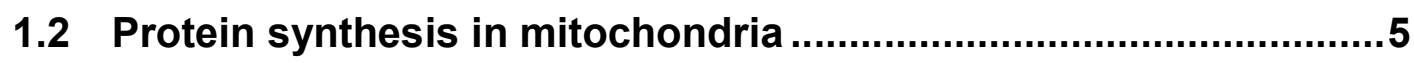

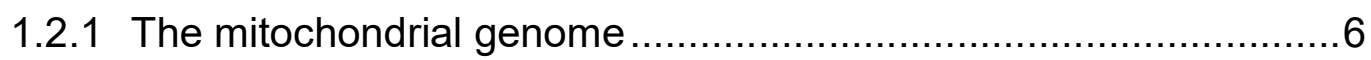

1.2.2 Transcription and maturation of mitochondrial RNA …...................

1.2.2.1 Stability of mitochondrial mRNA ............................................12

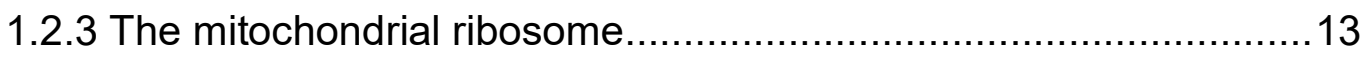

1.2.4 Mitochondrial translation and translation regulators......................18

1.2.4.1 COX1 translation in human mitochondria ..............................21

1.3 Biogenesis of the oxidative phosphorylation system ...................22

1.3.1 Biogenesis of the NADH Coenzyme Q oxidoreductase ................26

1.3.2 Biogenesis of the cytochrome $c$ oxidase ......................................34

1.3.2.1 Processing and assembly of COX1

1.3.2.1.a COX1 translation and early assembly factors ....................35

1.3.2.1.b The TIM21 shuttle and late MITRAC …............................36

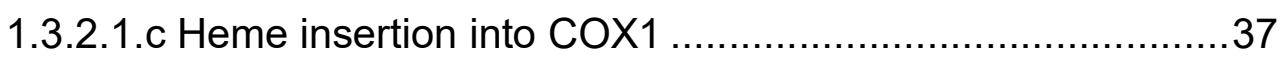

1.3.2.1.d Copper insertion into COX1 ............................................. 38

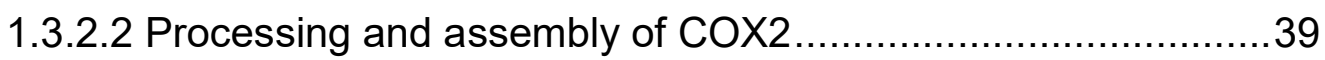

1.3.2.2.a COX2 membrane insertion and early assembly factors....39

1.3.2.2.b Copper insertion into COX2 .......................................... 40

1.3.2.3 Late stage assembly of the cytochrome $c$ oxidase ..................41 


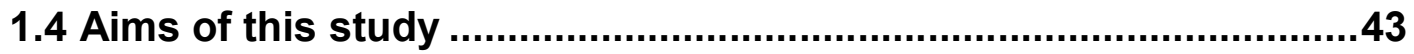

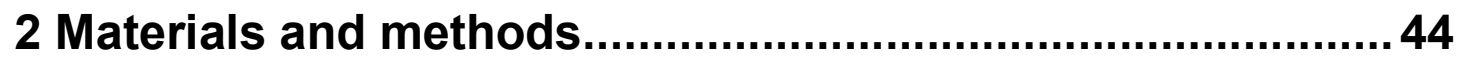

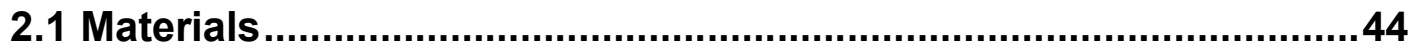

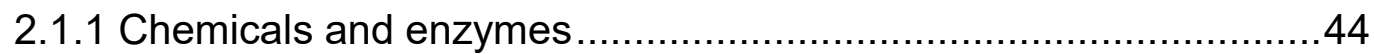

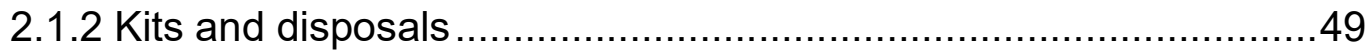

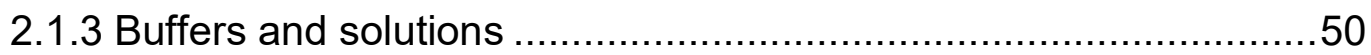

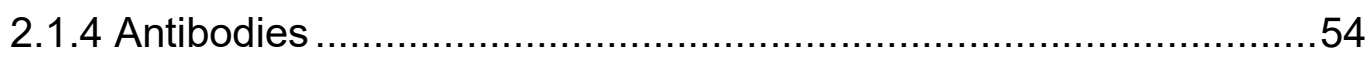

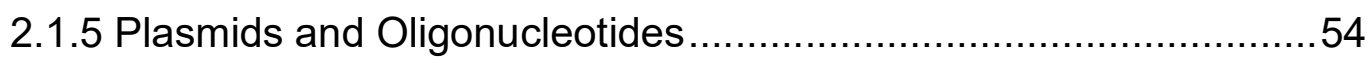

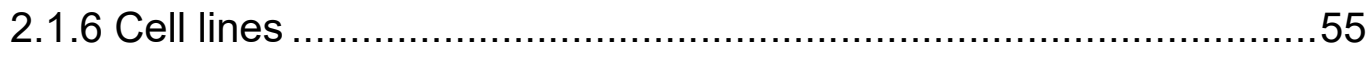

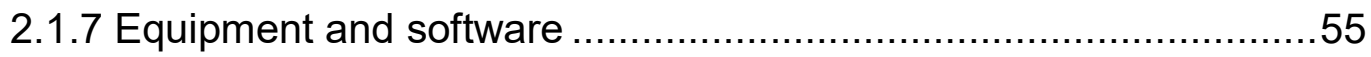

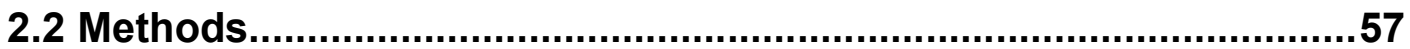

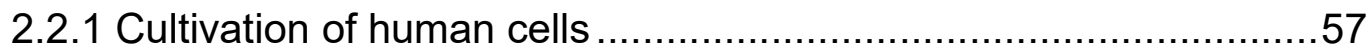

2.2.2 Generation of knockout cell lines using the CRISPR-Cas9 system57

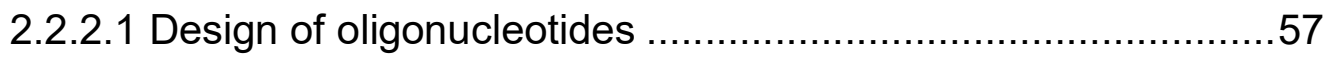

2.2.2.2 Cloning of oligonucleotides into the pX330 vector..................58

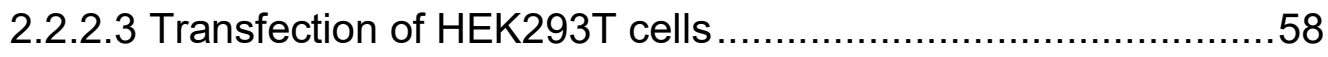

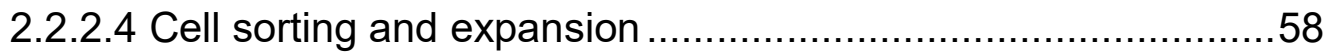

2.2.3 Labelling of newly synthesized mitochondrial proteins using $\left[{ }^{35} \mathrm{~S}\right]$

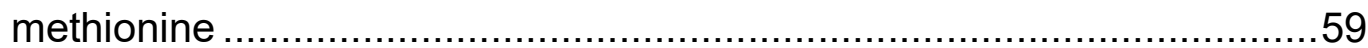

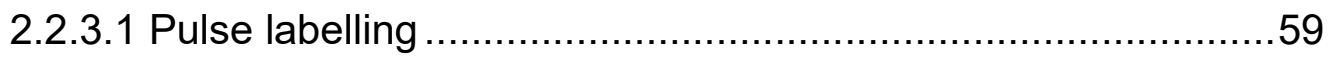

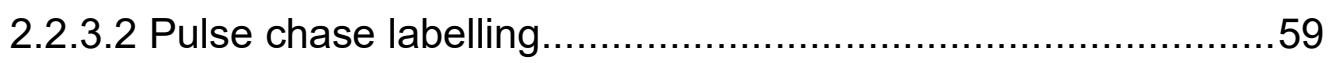

2.2.3.3 Pulse labelling with puromycin treatment ..............................59

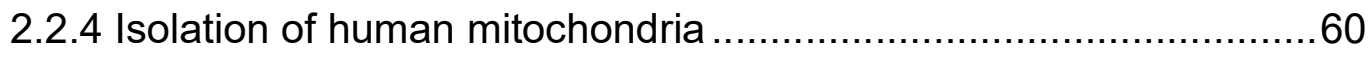

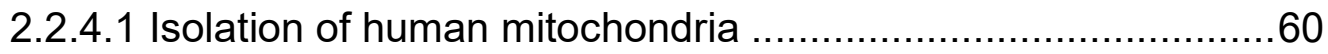

2.2.4.2 Large scale isolation of human mitochondria ..........................60

2.2.4.3 Isolation of human mitochondria for import ...........................61

2.2.5 Isolation of the crude mitochondrial ribosome fraction...................61

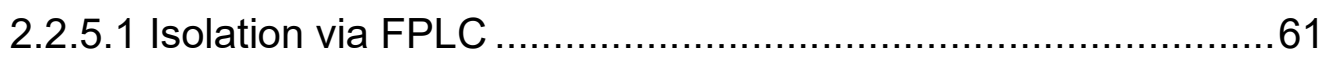

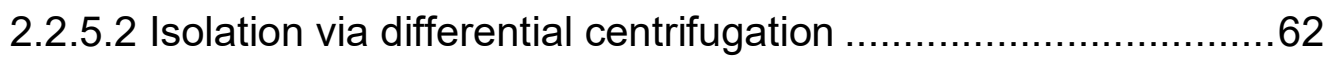

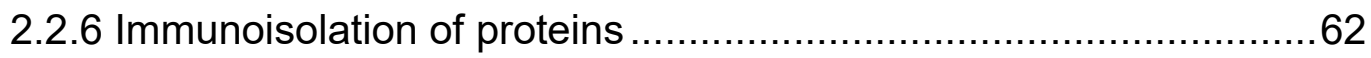

2.2.6.1 Immunoisolation with endogenous antibodies ........................62

2.2.6.2 Immunoisolation with Anti-FLAG M2 agarose beads...............63 
2.2.6.3 Immunoisolation with Anti-FLAG M2 agarose beads from the crude ribosome pellet for cryoEM

2.2.6.4 Immunoisolation with Anti-FLAG M2 agarose beads from the crude ribosome pellet for mass spectrometry...............................64

2.2.7 Import of mitochondrial proteins...........................................64

2.2.7.1 In vitro synthesis of radiolabeled precursor mitochondria proteins from plasmids

2.2.7.2 In vitro synthesis of radiolabeled precursor mitochondrial proteins from mRNA

2.2.7.3 Import and assembly of precursor mitochondrial proteins .65

2.2.8 Mass spectrometry analysis of the mitochondrial ribosome associated MITRAC complex.

2.2.9 Electron microscopy analysis of the mitochondrial ribosome associated MITRAC complex 66

2.2.10 Polymerase chain reaction (PCR) .66

2.2.11DNA isolation from $E$. coli. .66

2.2.12Quick isolation of human genomic DNA (gDNA) .....................66

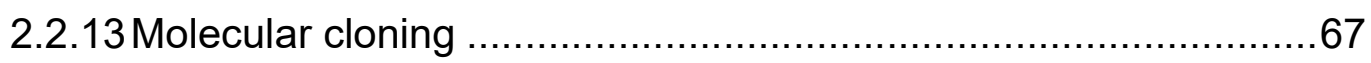

2.2.14 DNA electrophoresis and sequencing ...............................67

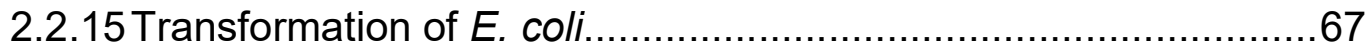

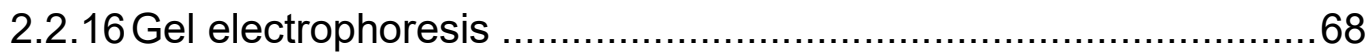

2.2.16.1 SDS-PAGE (glycin gels) ......................................... 68

2.2.16.2 SDS-PAGE (tris tricine gels) ...................................68

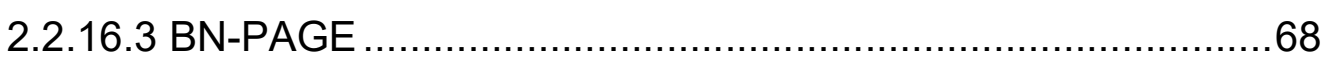

2.2.16.4 Western Blot........................................................... 69

2.2.16.5 Coomassie staining ............................................69

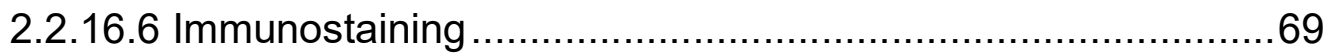

2.2.17 Determination of protein concentration.................................. 70

2.2.18 Fast lysate of HEK293 cells.............................................. 70

2.2.19 Determination of complex I and IV activity........................... 70

3 Results ............................................................................. 71

3.1 Structural analysis of the MITRAC complex associated with the translating mitochondrial ribosome 
3.1.1 C12ORF62 ${ }^{\text {FLAG }}$ co-purifies with the COX1 translating mitochondrial ribosome

3.1.2 Defining solubilisation conditions for the ribosome-associated

MITRAC complex.................................................................. 75

3.1.3 Separation of different forms of the MITRAC complex..................80

3.1.3.1 Separation of different forms of the MITRAC complex by size exclusion chromatography

3.1.3.2 Separation of different forms of the MITRAC complex via differential centrifugation .86

3.1.4 Mass spectrometric analysis of MITRAC ribo .90

3.1.5 Structure of MITRAC ribo .92

3.2 MITRAC15 is an assembly factor that links the biogenesis of mitochondrial complex I and complex IV .96

3.2.1 MITRAC15 is present in different complexes .97

3.2.2 MITRAC15 copurifies with newly synthesized mitochondrial proteins 100

3.2.3 MITRAC15 coisolates with fragments of newly synthesized mitochondrial proteins 101

3.2.4 Knockdown of TIM21 causes accumulation of MITRAC15 at the MITRAC complex 103

3.2.5 ACAD9 is needed for further assembly of the $\mathrm{P}_{\mathrm{P}}$-module. 105

3.2.6 Generation and analysis of a MITRAC $15^{-/-}$cell line 108

3.2.6.1 MITRAC15 affects ND2 synthesis but not complex I and complex IV activity 108

3.2.6.2 MITRAC15 ${ }^{-/-}$causes increase an in complex IV assembly....111

4.Discussion 114

4.1 Assessing the composition of the mitochondrial ribosomeassociated MITRAC.

4.1.1 Early assembly of complex IV ..........................................114

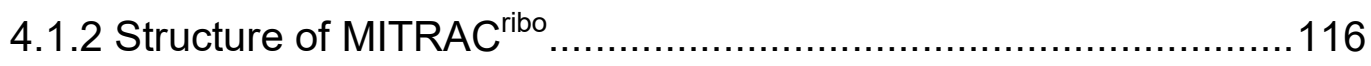

4.2 MITRAC15, a coordinator of complex I and IV assembly..............119

Summary and conclusion................................................... 126 
References.................................................................... 127

Acknowledgements............................................................ 151 


\section{List of figures}

Figure 1 General structure and different compartments of mitochondria.........3

Figure 2 The Oxidative phosphorylation system (OXPHOS). ........................ 4

Figure 3 Different forms of supercomplexes in human mitochondria............... 5

Figure 4 Organization of the mitochondrial genome ................................ 8

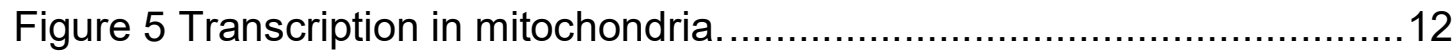

Figure 6 Structure of the human mitochondrial ribosome in two different orientations.

Figure 7 Structure of the central protuberance of the human mitochondrial ribosome.

Figure 8 The mitochondrial ribosomal exit tunnel. ......................................17

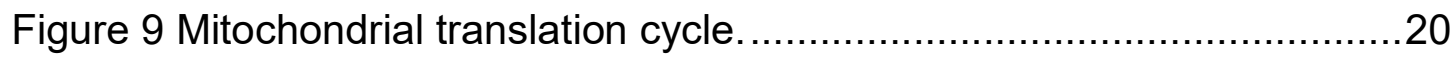

Figure 10 The mitochondrial complex IV contain subunits of dual origin. ......23

Figure 11 Structure of the mouse mitochondrial complex I. ........................27

Figure 12 Modular assembly of the mitochondrial complex I. ........................33

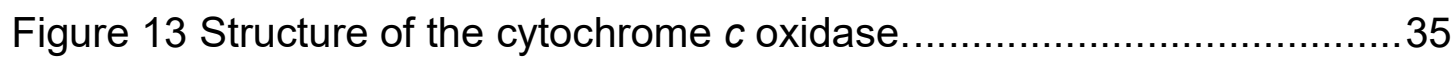

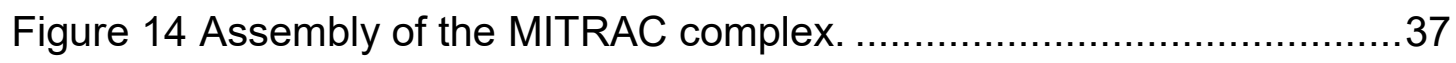

Figure 15 Maturation of COX2 ..........................................................

Figure 16 Putative model for the cytochrome $c$ oxidase assembly. .............42

Figure 17 C12ORF62 ${ }^{\text {FLAG }}$ copurifies with the large and the small subunit of

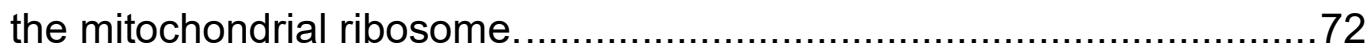

Figure $18 \mathrm{C} 12 \mathrm{ORF} 62^{\mathrm{FLAG}}$ copurifies with fragments of nascent COX1...........73

Figure 19 C12ORF62 ${ }^{\text {FLAG }}$ coisolated with a high molecular complex containing newly synthesized mitochondrial proteins.

Figure 20 PCC provides optimal solubilisation conditions for the isolation of C12ORF62 ${ }^{\text {FLAG }}$ associated complexes. ............................................77

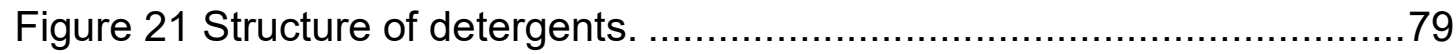

Figure 22 PCC provides strong solublization properties. .............................79

Figure 23 Isolation strategy for mitochondrial ribosome-associated MITRAC complex via C12ORF62 $2^{\text {FLAG }}$ 
Figure 24 Mitochondrial ribosome-associated C12ORF62 ${ }^{\text {FLAG }}$ can be purified by size exclusion chromatrgraphy (SEC)/immunoisolation tandem experiments.

Figure 25 Mass spectrometric analysis of MITRAC ribo isolation via C12ORF62 ${ }^{\text {FLAG }}$ reveals an accumulation of mitochondrial ribosomal proteins.

Figure 26 Purified MITRAC ribo shows aggregation under electron microscopy. .85

Figure 27 Isolation of the crude mitochondrial ribosome fraction via differential centrifugation.

Figure 28 Successful isolation of MITRAC ${ }^{\text {ribo }}$ with C12ORF62 $2^{\text {FLAG }}$ via sucrose cushion.

Figure 29 Electron microscopy picture of purified mitochondrial ribosomeassociated MITRAC complex shows mostly single particles. .90

Figure 30 Heat map of mass spectrometry analysis of isolated MITRAC ribo .91 Figure 31 Procedure for the selection of particles for cryoEM analysis of the isolated MITRAC ribo .93

Figure 32 Structure of MITRAC ribo .94

Figure 33 Structure comparison of the subspecies of the isolated large mitochondrial ribosome subunit with MITRAC. .95

Figure 34 MITRAC15 associated MITRAC12 and ACAD9 do not share the same complex...... .98

Figure 35 MITRAC15 exist in different independent complexes. .99

Figure 36 MITRAC15 copurifies with newly synthesized mitochondrial proteins.

Figure 37 MITRAC15 and not ACAD9 coisolates nascent chains of newly synthesized mitochondrial proteins. 102

Figure 38 Knockdown of TIM21 causes a higher association of MITRAC15 with the MITRAC complex. 104

Figure 39 ACAD9 depletion impairs assembly of the $\mathrm{P}_{\mathrm{P}}$-module, but does not affect ND2 binding of MITRAC15. 106

Figure 40 Nucleotide alignment of sequenced MITRAC15 $15^{--}$clone and WT exon 2 sequence. 108 
Figure 41 ND2 steady state levels and synthesis is decreased in MITRAC15 ${ }^{-/-}$ cells. 109

Figure 42 MITRAC15 $^{-/-}$cells display similar complex IV and complex I activities to WT cells.

Figure 43 MITRAC15 $15^{-/}$has no effect in the assembly of complex I but leads to increase in complex IV assembly. 112

Figure 44 Putative early assembly of mitochondrial complex IV. 116

Figure 45 TIM21 promotes the disassembly of MITRAC15 from MITRAC...121

Figure 46 Migration of MITRAC15 between complexes. 122

Figure 47 Early assembly of the $\mathrm{P}_{\mathrm{P}}-\mathrm{b}$ module. 124 


\section{List of Tables}

Table 1 List of chemicals used in this study........................................4 44

Table 2 List of Kits and disposals used in this study.............................49

Table 3 List of buffers and solutions used in this study...........................50

Table 4 List of plasmids used in this study.........................................54

Table 5 Oligonnucleoties used in this study....................................... 54

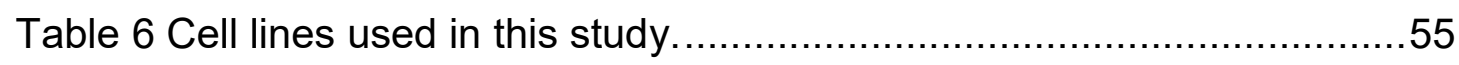

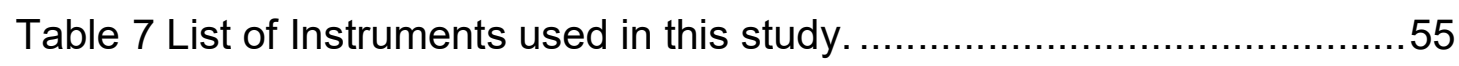

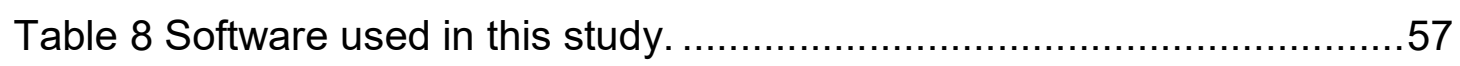




\section{List of abbreviations}

A

$\AA$

APS

ATP

AVO

BN-PAGE

$\mathrm{BNC}$

bp

BSA

C

cox

$\mathrm{CP}$

cryoEM

DMEM

DMSO

DNA

DDM

DIG

DMNG

dNTP

EDTA

ER

ERMES

FACS

$\mathrm{h}$

HEPES

HMG

HRP

HSP

G
Adenine

Ångström

Ammonium persulfate

Adenosine-5'-triphosphate

Antimycin A, valinomycin, and oligomycin

Blue native polyacrylamide gel electrophoresis

Binuclear center

Base pair

Bovine serum albumin

Cytosine

Cytochrome $c$ oxidase

C-entral protuberance

Cryo-electron microscopy

Dulbecco's Modified Eagle Medium

Dimethyl sulfoxide

Deoxyribonucleic acid

n-Dodecyl beta-D-maltoside

Digitonin

Decyl Maltose Neopentyl Glycol

2'-deoxynucleoside-5'-triphosphate

Ethylenediaminetetraacetic acid

Endoplasmic Reticulum

Endoplasmic reticulum - mitochondria encounter

structure

Fluorescence0activated cell sorting

Hour

4-(2-hydroxyethyl)-1-piperazineethanesulfonic acid

High mobility group

Horseradish peroxidase

Heavy-strand promoter

Guanine 


\begin{tabular}{|c|c|}
\hline GDP & Guanosine-5'-diphosphate \\
\hline GDN & Glyco-diosgenin \\
\hline GTP & Guanosine-5'-triphosphate \\
\hline IF & Initiation factors \\
\hline $\lg G$ & Immunoglobulin G \\
\hline IM & Inner mitochondrial membrane \\
\hline IMS & Intermembrane space \\
\hline IP & Immunopurification \\
\hline $\mathrm{kDa}$ & kilo Dalton \\
\hline LMNG & Lauryl Maltose Neopentyl Glycol \\
\hline LSP & Light-strand promoter \\
\hline LSU & Large subunit \\
\hline $\mathrm{MDa}$ & Mega Dalton \\
\hline MIA & $\begin{array}{l}\text { Mitochondrial intermembrane space assembly } \\
\text { machinery }\end{array}$ \\
\hline $\min$ & Minute \\
\hline MINOS & Mitochondrial inner membrane organizing system \\
\hline MITRAC & $\begin{array}{l}\text { Mitochondrial translation regulation assembly } \\
\text { intermediate of cytochrome } c \text { oxidase }\end{array}$ \\
\hline MOPS & morpholinopropanesulfonic acid \\
\hline MRG & Mitochondrial RNA granules \\
\hline MTERF & Mitochondrial Transcription Termination Factors \\
\hline mRNA & Messenger RNA \\
\hline $\mathrm{mt}$ & Mitochondrial \\
\hline $\mathrm{NADH}$ & Nicotinamide adenine dinucleotide \\
\hline $\mathrm{nm}$ & Nanometer \\
\hline nt & Nucleotide \\
\hline OBG & N-Octyl beta-D-Glucopyranoside \\
\hline OM & Outer mitochondrial membrane \\
\hline ОтС & Ornithine transcarbamylase \\
\hline OXPHOS & Oxidative phosphorylation \\
\hline $\mathrm{NADH}$ & Reduced nicotinamide adenine dinucleotide \\
\hline PAGE & Polyacrylamide gel electrophoresis \\
\hline PAM & Presequence translocase-associated motor \\
\hline
\end{tabular}




\begin{tabular}{|c|c|}
\hline PBS & Phosphate buffered saline \\
\hline PCC & $\begin{array}{l}\text { 4-trans-(4-trans-Propylcyclohexyl)-cyclohexyl } \alpha- \\
\text { maltoside }\end{array}$ \\
\hline PCR & Polymerase chain reaction \\
\hline $\mathrm{Pi}$ & Phosphate \\
\hline PK & Proteinase $\mathrm{K}$ \\
\hline PMSF & Phenylmethylsulfonyl fluoride \\
\hline POLRMT & Mitochondrial RNA polymerase \\
\hline PPR & Pentatricopeptide repeat \\
\hline PVDF & Polyvinylidene fluoride \\
\hline pur & Puromycin \\
\hline Q & Ubiquinone \\
\hline RNA & Ribonucleic acid \\
\hline ROS & Reactive oxygen species \\
\hline rpm & Rotations per minute \\
\hline RRM & RNA recognition motif \\
\hline rRNA & ribosomal RNA \\
\hline s & Second \\
\hline S. cerevisiae & Saccharomyces cerevisiae \\
\hline SAM & Sorting and assembly machinery of the outer membrane \\
\hline SILAC & Stable isotope labelling by amino acids in cell culture \\
\hline SDS & Sodium dodecyl sulfate \\
\hline SDS-PAGE & $\begin{array}{l}\text { Sodium dodecyl sulfate-polyacrylamide gel } \\
\text { electrophoresis }\end{array}$ \\
\hline SEC & Size exclusion chromatrgraphy \\
\hline SEM & Sucrose, EDTA, MOPS \\
\hline shRNA & Short hairpin RNA \\
\hline SSU & Small subunit \\
\hline $\mathrm{T}$ & Thymine \\
\hline TEMED & Tetramethylethylenediamine \\
\hline TH & Trehalose, Hepes \\
\hline TIM & Translocase of the inner mitochondrial membrane \\
\hline TM & Transmembrane \\
\hline TOM & Translocase of the outer mitochondrial membrane \\
\hline
\end{tabular}


tRNA

$\mathrm{U}$

UTR

$\mu \mathrm{m}$

VDAC

WT

$\Delta \Psi$
Transfer-RNA

Uracil

Untranslated regions

Micrometer

Voltage-dependent anion-selective channel

Wild type

Membrane potential 


\section{Abstract}

After a long time of coevolution between mitochondria and their eukaryotic host cells, the mitochondrial genome has diverged severely from it's $\alpha$ proteobacterial ancestors. Mitochondrial genes have mainly been transferred to the cytosolic genome and only a small number of highly hydrophobic core subunits of the oxidative phosphorylation system (OXPHOS) have been retained within the mitochondrial genome.

Since the OXPHOS consist of subunits of both mitochondrial and cytosolic origins, the translation of mitochondrial proteins has to be coordinated with the translation and import of proteins translated on cytosolic ribosomes to provide efficient assembly of the OXPHOS subunits.

One mechanism to regulate mitochondrial translation by cytosolic events is via regulators for mitochondrial translation. Such proteins are present during mitochondrial translation and can either interact with mitochondrial mRNAs, or associate with the mitochondrial ribosome. Even though many mitochondrial translation regulators are well characterized in yeast, little is known about them in human mitochondria.

In this thesis, I was able to isolate the ribosome-associated early assembly intermediate of the cytochrome $c$ oxidase, MITRAC, during the translation of COX1. Western blot and mass spectrometry analysis showed the presence of the insertase OXA1L within this complex and cryoEM analysis could localize the complex near the exit tunnel of the mitochondrial ribosome.

In a second part, MITRAC15, which was characterized as a MITRAC subunit and a subunit of the $P_{\mathrm{P}}-\mathrm{b}$ early assembly intermediate, was functionally characterized. MITRAC15 could be shown to be a positive translation regulator of ND2 and is the first identified protein to possibly copurify with nascent chains of mitochondrial complex I proteins after puromycin treatment. Furthermore, it was shown that MITRAC15 is able to stall the assembly of the cytochrome c oxidase and therefore, links complex I and IV biogenesis. 


\section{Introduction}

\subsection{Mitochondria structure and function}

Mitochondria are cell organellescommonly known as the "powerhouses of the cell". Although existing within eukaryote cells for over 2 billion years (Hedges et al., 2004), the earliest records that described mitochondrial like structures dates back to the 1840s. These structures were first recognized first by Richard Altmann in 1890 and were titled "bioblasts". The present name, formed by the Greek words "mitos" (thread) and "chondros" (granule), was introduced by Carl Benda in 1897 to describe the appearance of those organelles during spermatogenesis. The most established theory explaining the origin of mitochondria is the endosymbiosis theory. It states that mitochondria originate from an $\alpha$-proteobacterium (symbiont), which was ingested and thus annexed by an unidentified primitive eukaryotic host cell (Sagan, 1967; Yang et al., 1985).

The structure and physiology of mitochondria is strongly influenced by the origin of the organelle. Unlike other compartments in the eukaryotic cell, mitochondria possess two different membranes. The mitochondrial outer membrane (OM), whichseparates the organelle from the cytosol and the mitochondrial inner membrane (IM), which surrounds a compartment called the mitochondrial matrix. A second compartment exists between the $\mathrm{OM}$ and IM of mitochondria: the intermembrane space (IMS). While IM and matrix are compartments derived from the a-proteobacterialsymbiont, OMand IMS originate from the eukaryotic host cell (Frey and Mannella, 2000). Additionally there are foldings of the IM, creating secluded areas within the IMS called "cristae". Cristae are the localization of theoxidative phosphorylation system (OXPHOS), which is responsible for the most well known task of mitochondria: ATP synthesis(Stoldt et al., 2018) (Figure 1). 


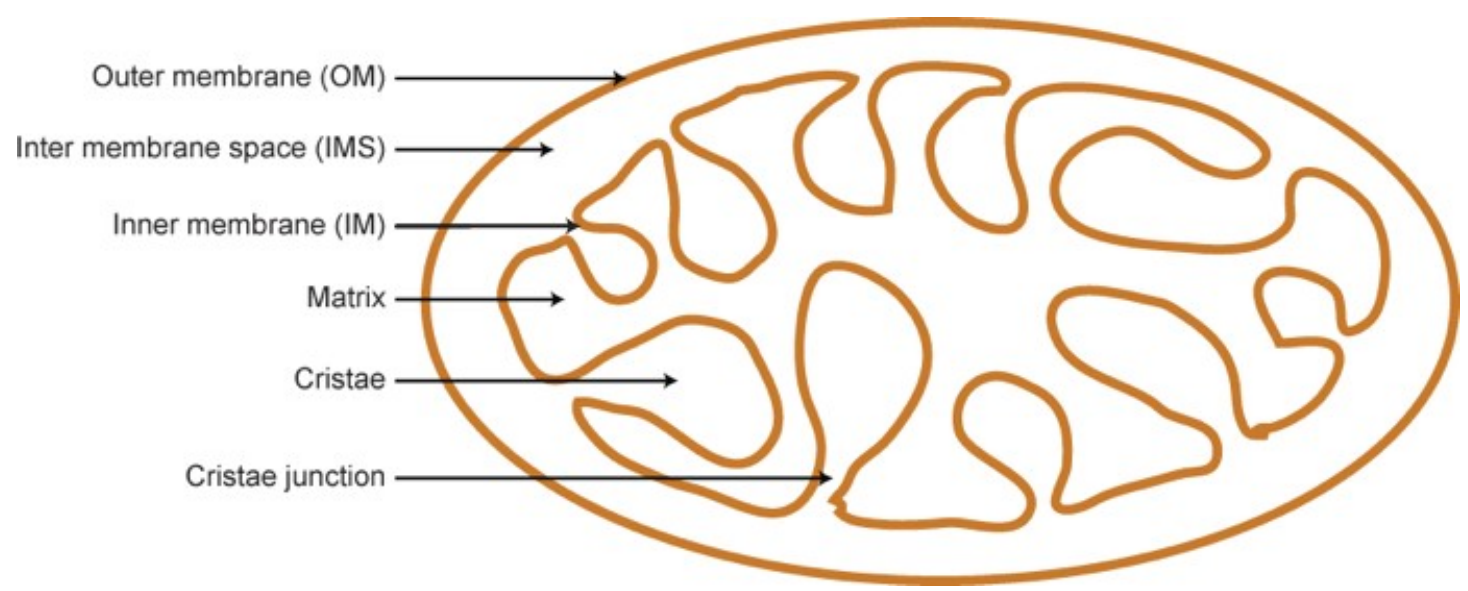

Figure 1 General structure and different compartments of mitochondria.

Besides providing the cell with energy in the form of ATP, mitochondria are also involved in a number of other important functions. They take part in catabolic metabolism pathways like $\beta$-oxidation of fatty acids(Eaton et al., 1996), the urea cycle(Mori et al., 1982), or the citric cycle(van der Bliek et al., 2017). Mitochondria are also involved in the biogenesis of iron sulfur clusters (Cardenas-Rodriguez et al., 2018),or different signalling pathways like calcium signalling and apoptosis(Tait and Green, 2012;Rizzuto et al., 2012). Due to the diversity of functions associated with mitochondria, they have been implicated in a variety of diseases.Diseases result from mutations in the mitochondrial genome and/or mutations in nuclear encoded mitochondrial proteins like Parkinson's disease (examples of affected proteins: Pink1, parkin)(Winklhofer and Haass, 2010) or Leigh syndrome (examples of affected proteins: LRPPRC, ND1 - 6) (Gerards et al., 2016).

\subsubsection{The Oxidative phosphorylation system}

One of the crucial traits all eukaryotes share is the ability to perform aerobic respiration. The reaction behind aerobic respiration is called oxidative phosphorylation and is carried out by the oxidative phosphorylation system (OXPHOS) in mitochondria, which consists of four complexes (I - IV), coupled to the ATP generating system (complex V).

The net chemical reaction of the OXPHOS is a rather simple one. Electrons provided by the citric cycle are used to reduce oxygen to water. 
Nevertheless, the process of electron transport is highly complex.The chemical energy stored in the electrons is used to create a proton gradient between the IMS and the mitochondrial matrix. The proton gradient can then be used to generate one of the basic energy providing compounds in eukaryotic cells: ATP.

Electrons are firstdelivered from the citric cycle, either via NADH to the NADH dehydrogenase (complex I), or via succinate to the Succinate-Q oxidoreductase (complex II)(Fiedorczuk et al., 2016;Cecchini, 2003). The oxidation of $\mathrm{NADH}$ by complex I causes a concomitant release of protons across the inner membrane and into the inner membrane space. In contrast, complex II has the same target for electron transfer but as the reaction releases less energy, it does not transfer protons. Both complexes transfer electrons to ubiquinone (Q). Reduced ubiquinone isoxidized by $Q$-cytochrome c oxidoreductase (complex III) and the electrons are transferred to cytochrome $c$ while protons are transferred to the IMS(Berry et al., 2000). The last complex participating in the chemical reaction is the cytochorome $c$ oxidase (complex IV). Here the electrons of the cytochrome $c$ are finally transferred to oxygen, reducing it to water and again transferring protons across the inner membrane(Castresana et al., 1994). The accumulation of protons into the inner membrane space, resulting from complex I, III and IV, generates a proton gradient which is used by the F1F0ATP synthase (complex V) to synthesize ATP (Mitchell and Moyle, 1968) (Figure 2).

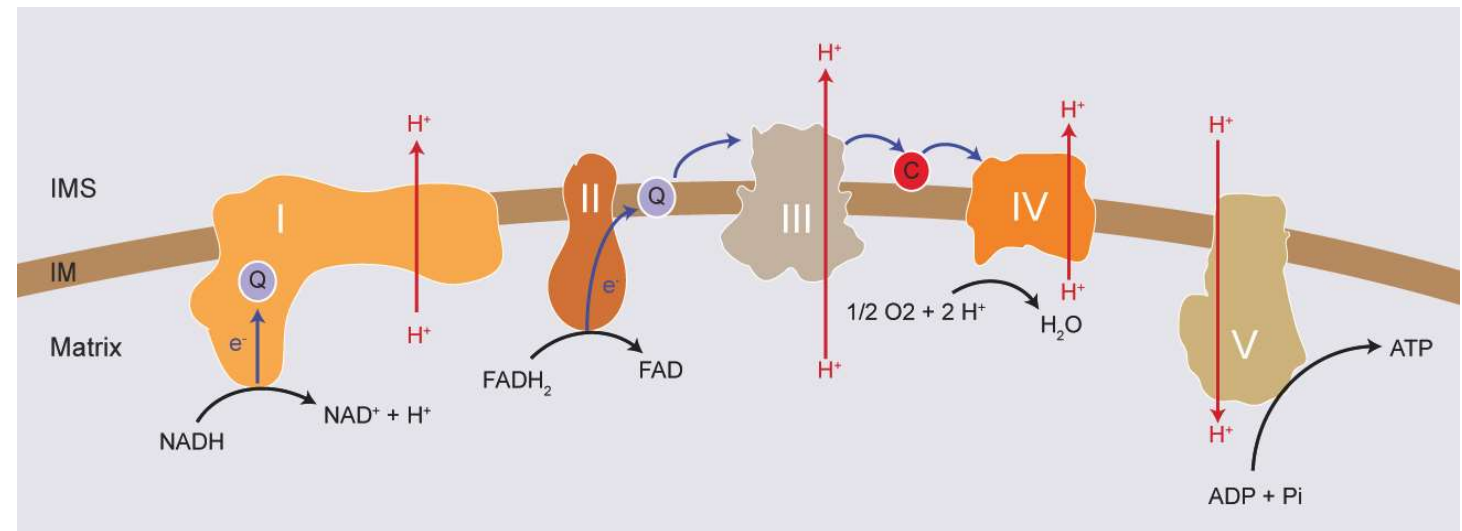

Figure 2 The Oxidative phosphorylation system (OXPHOS). Electrons are delivered to complex I and II by NADH and $\mathrm{FADH}_{2}$ respectively. The electrons are transported to complex IV to be used for the reduction of oxygen to water. Electron transport processes in the complexes I, III and IV generate a proton gradient, which is used by complex $\mathrm{V}$ to generate ATP 
OXPHOS complexes tend to associate with each other to form so called "supercomplexes". This was first reported by Schägger et al, whereby OXPHOS complexes and supercomplexes were resolved using BN-PAGE (Schägger and Pfeiffer, 2000). Heterogenic respiratory supercomplexes containing different OXPHOS complexes can be divided into 3 different species: I $+\mathrm{III}_{2}, \mathrm{III}_{2}+\mathrm{IV}_{1-2}, \mathrm{I}+\mathrm{III}_{2}+\mathrm{IV}_{1-4}$. Additionally, complex III and IV are able to form homodimers (Letts and Sazanov, 2017). Supercomplexes possibly enable a higher efficiency in electron transfer (Bianchi et al., 2004) and reduce the production of ROS in mitochondria (Lopez-Fabuel et al., 2016). In the case of human and mouse mitochondria, the stability of complex $I$ is dependant on supercomplex formation (Acin-Perez et al., 2004;Diaz et al., 2006). Also complex $V$ can form homodimers which have membrane shaping capabilities and are crucial for the formation of cristae (Hahn et al., 2016) (Figure 3).

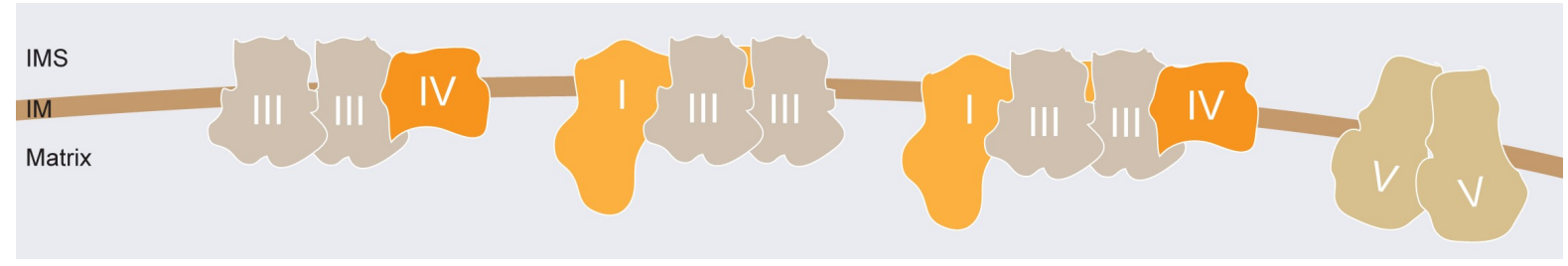

Figure 3 Different forms of supercomplexes in human mitochondria.

\subsection{Protein synthesis in mitochondria}

Due to their a-proteobacterial origin, mitochondria posses a functional system for transcription, translation and replication within the matrix. However, unlike the a-proteobacteria, mitochondria are not able to express the enzymes of the translation system and have to import nuclear encoded factors from the cytosol (Hällberg and Larsson, 2014). Most parts of the mitochondrial transcription and translation system have undergone a number of mitochondrial-specific adaptions, since their a-proteobacterial origin,which has led to the system they have today. 


\subsubsection{The mitochondrial genome}

With over 1000 identified mitochondrial proteins in mammals, and over 800 in yeast, mitochondria require a large variety of different proteins to maintain their functionality (Meisinger et al., 2008). While the a-proteobacterium was able to survive on its own and expressed a full set of essential elements with over 3600 genes (Lerma et al., 2015), the mitochondrial genome only encodes for a small subset of proteins, as well as functional RNA. Mitochondrial DNA was remodelled with their eukaryotic host cells in coevolutional events. Since those evolutional events occurred independently within lineages, mitochondrial genomes are highly diverse between species. Therefore, the number of mitochondrial genes can vary from over 100 genes in jakobids to only a few in parasitic protists (Burger et al., 2003).

Despite occurring independently between species, the evolution of the mitochondrial genome displays some common principles. Due to a mutation rate which is much higher than autosomes (45 times higher for human), mitochondrial genomic evolution is a relatively rapid process (Fu et al., 2014). The mutational events have lead to a general decrease of the mitochondrial genome, while the genes deleted in mitochondria were moved to the nucleus (Burger et al., 2003). Additionally, mitochondrial DNA has a high adenine, thymine content.This composition is suggested to be beneficial for the oxidative environment, because oxidative damage on $A$ and $T$ will lead to more tolerable mismatched base pairings(Martin, 1995). Two mitochondrial genes, which are present in all eukaryotic species, are COX1 and CYTb. Von Heijne et al. speculate that this is due to their strong hydrophobic nature which would restrict their import into mitochondria (Heijne, 1986).

Since mitochondrial DNA is exclusively maternally inherited (Kaneda et al., 1995) and hence, cannot undergo sexual recombination, it is highly susceptible to the accumulation of harmful mutations over time (Muller's ratchet, (Muller, 1964)). In mammals, several poorly understood mechanisms exist to counteract this development and ensure mitochondrial fitness: (a) Only a subset of the mtDNA pool is transferred to the next generation, because of a bottleneck phenomenon (Hauswirth and Laipis, 1982): (b) A purifying selection mechanism exist in the maternal germ line,which 
removesmtDNA mutations that lead to amino acid substitutions (Stewart et al., 2008): (c) A mechanism exist in developing embryos, which selects against mutations within mitochondrial tRNA (Stewart et al., 2008): (d) Females with high levels of mutations in mtDNA have decreased fertility.(Freyer et al., 2012).

The human mitochondrial DNA is double stranded, with a size of $16.6 \mathrm{~kb}$. It encodes 9 monocistronic and 2 bicistronic mRNAs, which translates into 13 core subunits of the OXPHOS complexes I, III, IV and V. Additionally, the mitochondrial DNA encodes for 2 ribosomal RNAs (12S and16S rRNA) and 22 t-RNAs (Hällberg and Larsson, 2014). The two strands of mitochondrial DNA differ in their base composition. One is rich in guanines and therefore possesses a higher molecular mass. Thus the two strands can be separated by density centrifugation in alkaline $\mathrm{CsCl}_{2}$ gradients and are labelled as the light (L) and heavy (H) strand historically (Berk and Clayton, 1974). Even though both the $\mathrm{L}$ and the $\mathrm{H}$ strand encode functional elements, most of the genetic information is stored in the $\mathrm{H}$ strand. The $\mathrm{L}$ strand only codes for 7 tRNAs, the replication primer for the $\mathrm{H}$ strand, and the complex I subunit ND6. All other proteins, tRNAs, as well as the 12S and 16S rRNA, are encoded by the $\mathrm{H}$ strand (Hällberg and Larsson, 2014). The heavy-strand promoter (HSP) and the light-strand promoter (LSP) are both localized in a non-coding area of the mitochondrial DNA that also contains the origin of replication for the $\mathrm{H}$ strand (Gustafsson et al., 2016). 


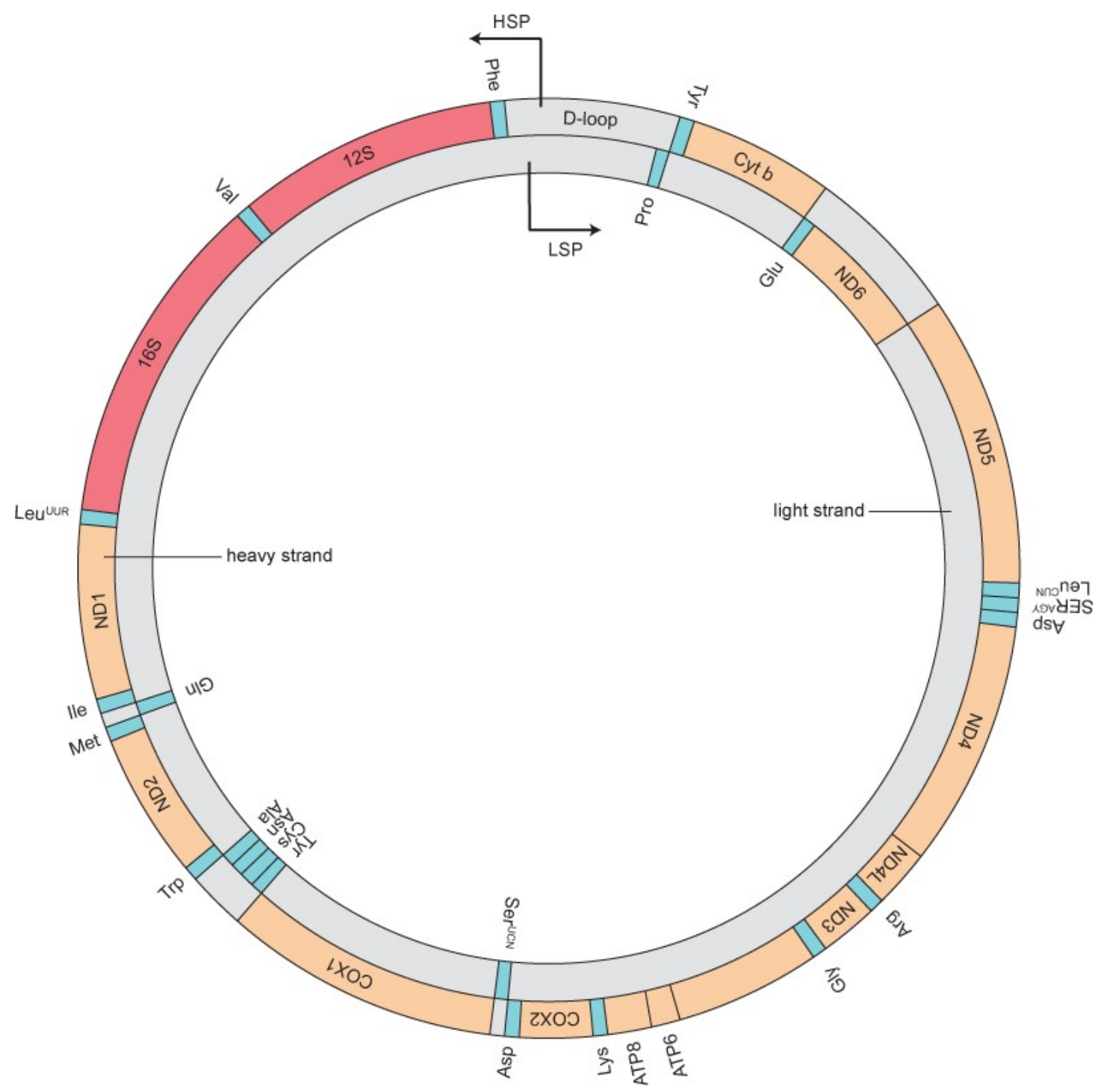

Figure 4 Organization of the mitochondrial genome.The non-coding regions are coloured in grey. The regions coding for the mitochondrial rRNA are coloured in red. The regions coding for tRNAs are coloured in blue. The regions coding for mitochondrial proteins are coloured in orange.

Similar to bacterial DNA (Dame et al., 2012), mtDNA are densely packed into small structures called nucleoids. In mammals, mtDNA molecules with a length of approximately $5 \mu \mathrm{m}$ (Nass, 1966) are packed into nucleoids with a diameter of around $100 \mathrm{~nm}$ (Kukat and Larsson, 2013). These nucleoids contain an average number of 1.4 copies of mtDNA molecules (Kukat et al., 2011). The major player in the maintaining the mtDNA nucleoid structure is a protein named mitochondrial transcription factor A (TFAM). TFAM is a protein that belongs to the high mobility group (HMG)-box family, which enables it to bind DNA (Malarkey and Churchill, 2012). It is able to pack mtDNA into compact nucleoids alone via a cooperative binding mode, where one molecule of TFAM binds to 16 - $17 \mathrm{bp}$ (Bogenhagen, 2012) and the 
condensation of the DNA molecule increase the more TFAM interacts with it. Beside its function in mtDNA structure, TFAM is also required for the initiation of mitochondrial transcription, described below (Yakubovskaya et al., 2014).

\subsubsection{Transcription and maturation of mitochondrial RNA}

Mitochondrial transcription is carried out by the mitochondrial RNA polymerase (POLRMT) and bears little similarity to the process in mammalian or bacterial transcription, but rather resembles transcription in the bacteriophage T7. The mammalian POLRMT is related to the RNA polymerase encoded by the bacteriophage T7 (RNAP) and possesses an Nterminal domain (amino acids 368 - 647) with similarity to the AT-rich recognition loop in T7 RNAP, which is needed for promoter binding (Ringel et al., 2011). POLRMT possesses two other characteristic domains: the Cterminal catalytic domain (amino acids 647 - 1230) (Ringel et al., 2011) and the N-terminal extension (amino acids NTE, $42-368$ ), a unique feature within the POLRMT that has an unknown function (Schwinghammer et al., 2013). Unlike the T7 RNAP, the N-terminal domain (amino acids 368 - 647) in POLRMT does not participate in promoter binding, but rather functions during transcription elongation by separating RNA from DNA (Schwinghammer et al., 2013).

The initiation of mitochondrial transcription takes place in the promoter regions containing LSP and HSP by the formation of an initiation complex on one of the promoters. The initiation complex consists of 3 components; TFAM, which is responsible for creating a characteristic U-turn in the DNA that facilitates POLRMT binding (Yakubovskaya et al., 2014); the mitochondrial transcription factor B2 (TFB2M), which forms part of the catalytic site together with POLRMT and is required for structural changes in the initiation complex (Posse et al., 2014); and finally, POLRMT itself. Once elongation starts, the mitochondrial transcription elongation factor (TEFM) interacts with the catalytic C-terminal domain of POLRMT (Minczuk et al., 2011). TEFM stimulates the interaction of POLRMT with the DNA:RNA template, assists in bypassing highly structured RNA, and stimulates transcription past oxidative 
lesions, which can cause premature termination of transcription (Posse et al., 2015;Agaronyan et al., 2015). The mechanism of mitochondrial transcription termination is still enigmatic. A family of potential termination factors that facilitate DNA unwinding were identified and are known as the Mitochondrial Transcription Termination Factors (MTERFs). However, only MTERF1 can be loosely related to translation termination (Yakubovskaya et al., 2010). The other 3 known members of this family (MTERF $2-4$ ) are associated with other functions, like negative transcription regulation (MTERF3) (Park et al., 2007), or mitochondrial monosome assembly (MTERF4-NSUN4 heterodimer) (Metodiev et al., 2014). MTERF2 shows association with the mitochondrial nucleoid but it's function still has to be determined (Pellegrini et al., 2009).

Both the $\mathrm{L}$ and the $\mathrm{H}$ strand are each transcribed into a large polycistronic and intron-free RNA molecule, which is then further processed to release the functional elements. The mitochondrial mRNA and rRNA are mainly flanked by tRNAs, which are also the initiation points for processing. The mRNA and rRNA are released from the flanking tRNAs according to the widely accepted tRNA punctuation model (Ojala et al., 1981). Processing of the polycistronic transcript happens in small distinct locations referred to as mitochondrial RNA granules (MRG) (Jourdain et al., 2013). These granules contain the 5'processing machinery known as RNase $P$, which consist of 3 subunits (MRPP1 - 3). While MRPP1 and MRPP2 form a stable complex (Jourdain et al., 2013), MRPP3 only interacts transiently with this complex (Rossmanith, 2008). The MRPP1-MRPP2 complex performs the N1-methylation of the 9th position in the tRNA (m1R9), which is critical for obtaining the tRNA specific cloverleaf fold (Vilardo et al., 2012). During this modification, MRPP3 will join the RNase P complex and trim the 5' end of the tRNA (Rossmanith, 2008). The 3' processing subsequently occurs outside of the MRGs and is carried out by the RNase Z, ELAC2 (Jourdain et al., 2013;Brzezniak et al., 2014). Once processed, the CCA acceptor stem is added onto the tRNA, by the CCA-adding enzyme (Reinhard et al., 2017). During the whole maturation procedure, the tRNA is associated with the MRPP1-MRPP2 complex, which can enhance ELAC2 enzyme activity (Reinhard et al., 2017).

Besides the N1-methylation at position 9, mitochondrial tRNA also harbours further post transcriptional modifications. Those modifications either enhance 
the overall structure of the tRNA, or are crucial for codon-anticodon interaction. A common modification that facilitates codon-anticodon interaction during decoding is a methyl-taurine modification located on a Uracil base at the C5 wobble position (Kurata et al., 2003). A further example for a structurally relevant modification is the pseudouridination of position $\mathrm{U} 27$ and U28 in several tRNAs (tRNA ${ }^{\mathrm{K}}, \operatorname{tRNA}^{\mathrm{M}}$, tRNA ${ }^{\mathrm{L}}$, tRNA ${ }^{\prime}$, and $\operatorname{tRNA}^{\mathrm{D}}$ ), which serve to stabilize and order of the anticodon stem loop(Agris, 2008). Still, one of the most crucial modifications happens at the C34 position of $\operatorname{tRNA}{ }^{\mathrm{M}}$. Besides decoding for the universal start codon AUG and AUA(Anderson et al., 1981), which encodes for isoleucine outside mitochondria, in both translation initiation and elongation, the $\operatorname{tRNA}^{\mathrm{M}}$ has also to be able to decode the mitochondrial specific start codons AUU and AUC(Fearnley and Walker, 2002). Formylation at the position $C 34$ in $\operatorname{tRNA}^{\mathrm{M}}$ was shown to be crucial for the recognition of the AUA codon(Takemoto et al., 2009) and is also considered assist with the AUU and AUC codon(Boos et al., 2016).

In most cases, the mitochondrial mRNA is released as a consequence of tRNA processing. However, exceptions include, ND5/CYTB and ATP6+ATP8/COX3, which are not flanked by tRNA. The mechanism underlying their processing remains enigmatic (Hällberg and Larsson, 2014). Once released, mRNAs undergo posttranscriptional modifications to stabilize them and initiate translation. Unlike cytosolic mRNA, mammalian mitochondrial mRNA often lacks, or has an extremely short 5' and 3' UTR (Hällberg and Larsson, 2014). Furthermore, the typical 7-methylguanosine cap at the 5 ' end of the mRNA is not present, which is essential for translation initiation in lower eukaryotes (Cowling, 2009). The only known modification of mitochondrial mRNA are short polyA tails (45 - $50 \mathrm{nt}$ ) at the 3' end (Ojala et al., 1981). Those polyA tails are present in all mitochondrial mRNAs, with the exception of ND6, which is immediately competent for translation after liberation from the polycistronic primary transcript of the $L$ strand (Temperley et al., 2010). Yeast mitochondrial mRNA differs from mammalian in the context of UTR's, since mitochondrial mRNA in yeast do contain 5' and 3' UTR, which interacts with a number of translation factors (Dennerlein et al., 2017). 


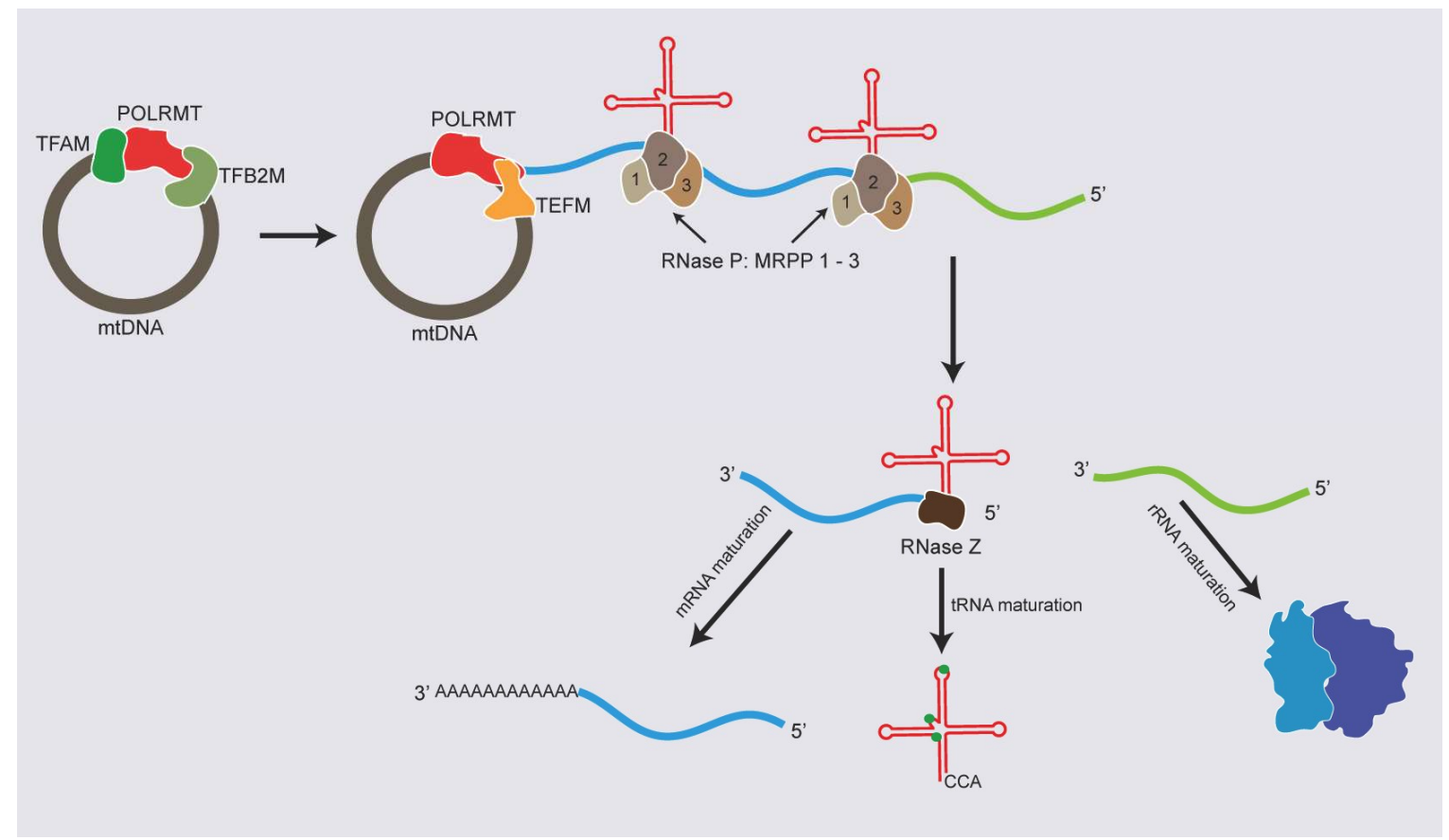

Figure 5 Transcription in mitochondria. Mitochondrial DNA is transcribed by the only RNA polymerase present in mitochondria: POLRMT. After transcription, The RNase P complex and the RNase $Z$ process the polycistronic RNA at the positions of the tRNA into monocistronic mRNA or rRNA. mRNA, tRNA and rRNA undergo different maturation processes before they can fulfil their function.

\subsubsection{Stability of mitochondrial mRNA}

Polyadenylation within mitochondrial mRNA is tightly coupled with the stability of the transcript and is regulated by an interplay between the mitochondrial polyadenylation polymerase (mtPAP) (Nagaike et al., 2005) and two enzymes characterized as part of the mitochondrial degradosome: the human mitochondrial helicase, SUV3, and the polynucleotide phosphorylase (PNPase), a 3' to 5' phosphate-dependent exoribonuclease (Borowski et al., 2012). While mtPAP is known to carry out the polyadenylation of mRNAs and the SUV3/PNPase the degradation of mitochondrial mRNA, SUV3 is also able to form a complex with mtPAP to enhance its efficiency (Wang et al., 2014). Additionally, SUV3 is able to act as a bridge and form a complex with both mtPAP and PNPase. This complex with antagonistic enzymes was shown to be able to modulate the length of the polyA tail of mitochondrial mRNA in vitro, depending on the Pi/ATP ratio within the system (Wang et al., 2014). 
Another more indirect player in mitochondrial mRNA stability is the protein pair, LRPPRC/SLIRP. These two proteins form a heterodimer via an interaction between the 3 pentatricopeptide repeat (PPR) motifs of LRPPRC and the RNA recognition motif (RRM) of SLIRP (Spåhr et al., 2016). The heterodimeric complex enhances mRNA stability by impairing the degradation via SUV3/PNPase and promoting the activity of mtPAP (Chujo et al., 2012). While the LRPPRC harbours mRNA binding ability and prevents the accumulation of secondary structures, SLIRP does not bind mRNA despite the RRM domain and is rather necessary to stabilize LRPPRC (Spåhr et al., 2016), (Siira et al., 2017).

\subsubsection{The mitochondrial ribosome}

Mitochondrial ribosomes are largely different from their bacterial ancestors. Since the evolutional events took place individually in different lineages, mitochondrial ribosomes display specific differences. Despite this, general trends are also noticeable for most of the lineages. (a) Until the divergence of the metazoan, there was a trend in the expansion of mitochondrial rRNA.Nevertheless, the evolution of the metazoan was accompanied by a reduction of mitochondrial rRNA (van der Sluis et al., 2015). This feature was most dominant within mammals, who posses a mt-rRNA length of only 2532 $\mathrm{bp}$, which is about half the length of bacterial rRNA (4458 bp) or yeast (4941) mt-rRNA (Greber and Ban, 2016). (b) In contrast to the declining mitochondrial rRNA, there was an expansion in the number of mitochondrial ribosomal proteins, which increased from 54 in the bacterial ribosome to 82 in the mitochondrial ribosomes of mammals and yeast (Greber and Ban, 2016). Research into mitochondrial ribosomes made crucial progress upon the generation of structural data by cryoEM. The first structure of the $55 \mathrm{~S}$ monosome was provided by the lab of Rajendra K. Agrawal (Sharma et al., 2003), with a resolution of $12-14 \AA$. With the improvement of cryoEM technology over the years, mitochondrial ribosomal structures were obtained with a much higher resolution (Bai et al., 2015), including a $4.9 \AA$ resolution model of the structure of the mammalian 395 mitochondrial ribosomal large 
subunit (Greber et al., 2014) and a $3.2 \AA$ structure of the yeast $54 S$ mitochondrial ribosomal large subunit (Amunts et al., 2014). Today, atomic structures also exist for monosomes of mitochondria in human (3.5 $\AA$ (Amunts et al., 2015)) (Figure 6), pig (3.8 $\AA$ (Greber et al., 2015a)) and yeast (3.3 $\AA$ (Desai et al., 2017)).

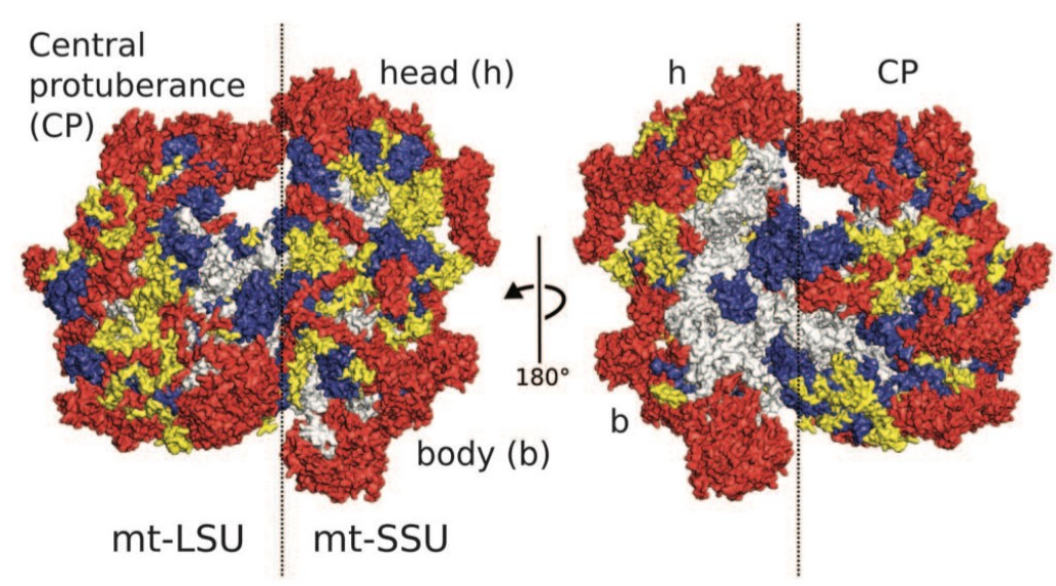

Figure 6 Structure of the human mitochondrial ribosome in two different orientations. Taken from (Amunts et al., 2015).

One of the most striking differences in the mitochondrial ribosome compared to its bacterial ancestor is the loss of the $5 \mathrm{~S}$ rRNA (Nierlich, 1982). The $5 \mathrm{~S}$ rRNA is a conserved architectural feature of both the bacterial, archaeal and eukaryotic ribosome and acts as a structural scaffold for the formation of the central protuberance (CP) with ribosomal proteins (Voorhees et al., 2009). The CP is characterized as an area of the large ribosomal subunit that carries out important functions, like the mediation of intersubunit contacts to the small subunit for movement coordination during translation and for interaction with tRNAs in the intersubunit space (Dinman, 2005). Despite the missing 5S rRNA, the CP still exist within the mitochondrial ribosome, which raises the question about its organization (Amunts et al., 2015). The answer to this phenomenon was provided by cryoEM generated structural data, which showed a structural tRNA in the mammalian CP at a position similar to one $5 \mathrm{~S}$ rRNA domain in bacteria (Greber et al., 2014;Brown et al., 2014). This tRNA was identified to be tRNA ${ }^{\text {Phe }}$ in porcine mitochondria (Greber et al., 2014) and tRNA $^{\mathrm{Val}}$ in human mitochondria (Brown et al., 2014).Both tRNAs flank the $12 \mathrm{~S}$ rRNA, and their processing is involved in the release of both $16 \mathrm{~S}$ and $12 \mathrm{~S}$ 
rRNA (Figure 4). Similar to the 5S rRNA, the structural tRNA also interacts with mitochondrial ribosomal proteins in the $\mathrm{CP}$, especially with a mitochondrial ribosomal protein in the large subunit, uL18m (Figure 7). Notably, there is a preserved interaction of the bacterial homolog uL18 with the 5S rRNA (Greber and Ban, 2016). Interestingly, the 5S rRNA is not replaced by a structural tRNA in yeast, but with an expansion of already existing rRNA(Amunts et al., 2014).

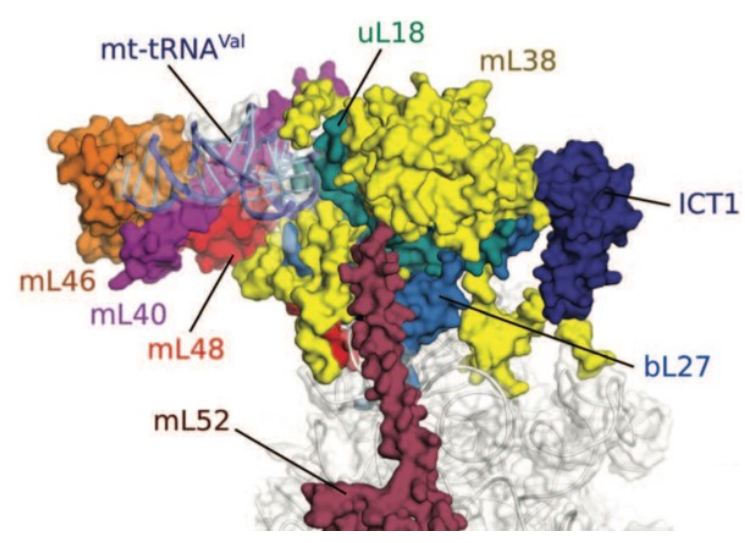

Figure 7 Structure of the central protuberance of the human mitochondrial ribosome. Taken from (Brown et al., 2014).

A second distinct structural adaption of the mitochondrial ribosome is the polypeptide exit tunnel (Figure 8). This tunnel is the path along which nascent chains pass in order to be released from the ribosome. Folding of the polypeptide chain may already take place during this process. Unlike eukaryotic or bacterial ribosomes, which have to adapt to a wide range of different proteins, the mitochondrial ribosome evolved to adapt for the synthesis of membrane proteins, to which all mitochondrial-translated proteins belong. The evolution of the exit tunnel was facilitated because the former rRNA structures in bacteria were exchanged for mitochondrial ribosomal proteins, which have the added ability to establish new properties for the surface of the exit tunnel. Indeed, the protein residues facing the surface of the mitochondrial ribosomal exit tunnel are of hydrophobic nature and show specific interactions with the nascent chain, thereby mimicking the native, hydrophobic environment of membranes(Brown et al., 2014). While the exit tunnel of the human mitochondrial ribosome still shows similarity to its bacterial successor (Brown et al., 2014), the exit tunnel in yeast mitochondrial 
ribosomes has completely changed position and is localized $3.5 \mathrm{~nm}$ away from its position in bacterial ribosomes. This relocalization is attributed to 2 structural changes in the yeast mitochondrial ribosome; loss of helix 24 of the $18 S$ rRNA, which creates a new passage for the nascent chain into the solvent; and extension of the mitochondrial ribosomal protein uL23, which blocks the conserved path in the ribosome (Amunts et al., 2014). A further characteristic feature of the mitochondrial ribosome is located at the end of the exit tunnel. A mitochondrial ribosomal protein present in mammals, $\mathrm{mL} 45$, possesses $\mathrm{C}$-terminal helices that extend into the inner boundary membrane (Greber et al., 2014). By mediating the membrane association of the mitochondrial ribosome, $\mathrm{mL} 45$ plays a major role in facilitating the membrane insertion of mitochondrial ribosomal transcripts (Greber et al., 2014Englmeier; et al., 2017). Membrane association is a general crucial feature of mitochondrial ribosomes, which is emphasized by the existence of a yeast homolog of $\mathrm{mL} 45$, known as Mba1. Even though it is not permanently associated with the mitochondrial ribosome, Mba1 mediates the membrane association of translating mitochondrial ribosomes and plays a role as a mediator between the nascent chain and the insertion machinery of the inner mitochondrial membrane (Ott et al., 2006; Amunts et al., 2014). 
A

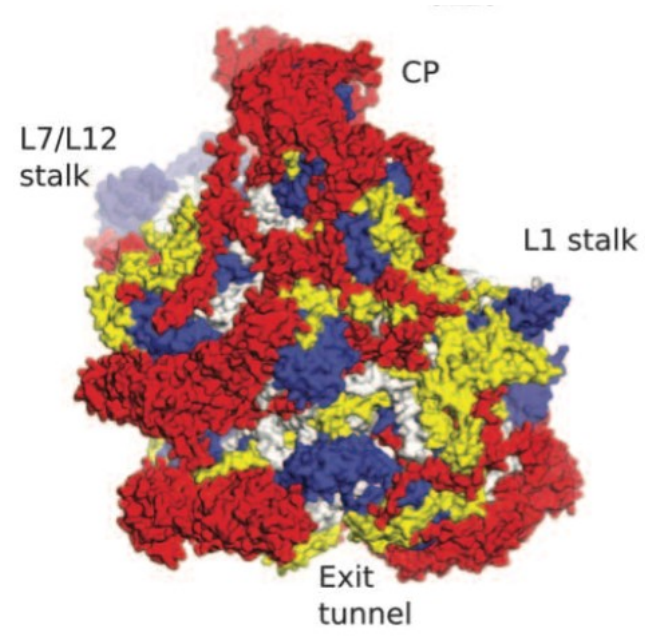

B

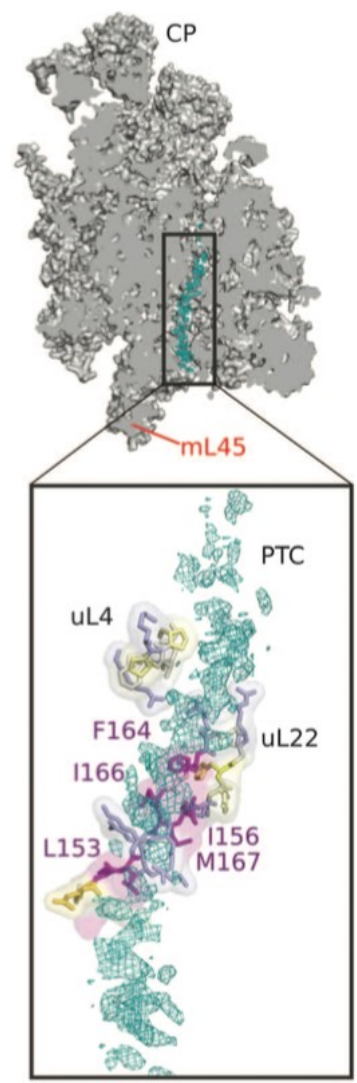

Figure 8 The mitochondrial ribosomal exit tunnel. A) Localization of the exit tunnel. B) Structure of the exit tunnel. A) and B) taken from (Brown et al., 2014).

Despite the broad spectrum of adaptations during the evolution of the mitochondrial ribosome, the catalytic core still consists of conserved rRNA, with only few mitochondrial-specific elements. Still, there are small variations within the $A, P$ and $E$ sites, which affect mitochondrial translation. Due to the sequence-length variations of the $\mathrm{D}$ - and T- stems of mitochondrial tRNA, mammalian mitochondrial ribosomes lack the elements to interact with those regions (Helm et al., 2000). The missing elements are uL25m and the rRNA helix H38 (A-site finger) in the A-site, and uL5m and H84 in the P-site (Brown et al., 2014). In the P-site, mitochondrial ribosomes posses a distinct structural element called the $\mathrm{P}$-site finger, which originates from the $\mathrm{CP}$ and may serve to compensate for the loss of ribosome-tRNA interaction caused by the missing A-site finger (Greber et al., 2015a). The E-site of mitochondrial ribosomes is rather unmodified in comparison with its bacterial predecessor and displays a CCA binding pocket, which is conserved in both sequence and structure. Another strongly conserved element is the decoding site, localized 
in the small mitochondrial $28 \mathrm{~S}$ subunit (Koch et al., 2015). The key structural elements of the decoding site show the same interactions within the codonanticodon helix, suggesting a highly conserved mechanism between mitochondrial ribosomes and their bacterial ancestors (Greber et al., 2015a).

\subsubsection{Mitochondrial translation and translationregulators}

Translation is a universally conserved mechanism that occurs in all forms of life. The translational process can be summarized into 3 different steps; (a) initiation, where the ribosome locks onto the start codon of the mRNA; (b) elongation, where the peptide is formed by the addition of amino acids and (c) termination, where the polypeptide chain is released from the ribosome and the ribosome is subsequently recycled. Each of these steps is facilitated by specific translation factors and even though the translation factors in mitochondria often have homologues in bacteria, adaptation was necessary because of the changes in mitochondrial mRNA, ribosome and the genetic code.

In bacteria, translation initiation is aided by 3 initiation factors (IF1, IF2, IF3) and the bacterial rRNA of the SSU interacts with the highly conserved ShineDalgarno sequence on mRNA to recognize the start of translation (Gualerzi and Pon, 2015). Mammalian mitochondrial mRNAs do not posses this feature, or a 5' cap structure, but the start codon is identified closest to the 5' end (Zhang et al., 2015). A lacking secondary structure at the 5' end of mitochondrial mRNA is believed to facilitate codon recognition (Jones et al., 2008). Furthermore, only IF2 and IF3 have a known mitochondrial homologue, while IF1 is missing (Liao and Spremulli, 1990;Koc and Spremulli, 2002; Cummings and Hershey, 1994). IF3 promotes dissociation of the mitochondrial ribosome and prevents association of the mitochondrial monosome. For translation initiation, GTP-bound IF2 assists in tRNA ${ }^{\text {Met }}$ placement in the $\mathrm{P}$ side of the SSU, displacing IF3 and allowing the LSU to bind. The formation of the active monosome causes the hydrolysis of GTP and the release of IF2 (Christian and Spremulli, 2012). 
The process of elongation is the most conserved of the mitochondrial translation steps. Elongation is accompanied by 3 elongation factors, all with bacterial homologues; EF-Tu, in complex with GTP, provides the mitochondrial ribosome with aminoacyl-tRNA; EF-Ts exchange the GDP bound with EF-Tu to GTP, after it is released by the ribosome; EF-G1 functions as a catalyst in the coordinated movement of mRNA and tRNA during translocation (Schwartzbach and Spremulli, 1989;Bhargava et al., 2004). Despite the conservation of the elongation process, bacterial elongation factors are not able to function with the mammalian mitochondrial ribosome (Eberly et al., 1985).

In contrast to the elongation process, mitochondrial termination is not as well conserved from bacteria. While bacteria have three stop codons (UGA, UAA, UAG) (Korkmaz et al., 2014), mitochondria only utilize two (UAA, UAG), with UGA coding for tryptophan. These two stop codons are supplemented with two additional non canonical stop codons, AGA/AGG (Lind et al., 2013). The bacterial stop codons are recognized by two release factors (RF1, RF2) (RaySoni et al., 2016), of which only RF1 has a mitochondrial homologue (mtRF1a) (Lee et al., 1987). However, mitochondria posses an additional release factor, ICT1, which is able to recognize the stop codons (Akabane et al., 2014). Interestingly, ICT1 functions as both as a subunit of the mitochondrial ribosome and, in its non bound form, as a release factor for mitochondrial translation (Richter et al., 2010). Furthermore, there are two stop codon-independent release factors (mtRF1, C12ORF65), which are suggested to assist in the recycling of stalled ribosomes (Huynen et al., 2012), (Antonicka et al., 2010). After the release of the translated protein, the ribosome recycling factor (RRF1) and a specific homologue of the EF-G: EFG2 associates with the monosome and cause its disassembly in the free small and large subunit(Tsuboi et al., 2009). The free mitochondrial ribosomal subunits are thus recycled and IF3 can associate with the small subunit again (Christian and Spremulli, 2009). The process of mitochondrial translation is summarized below (Figure 9). 


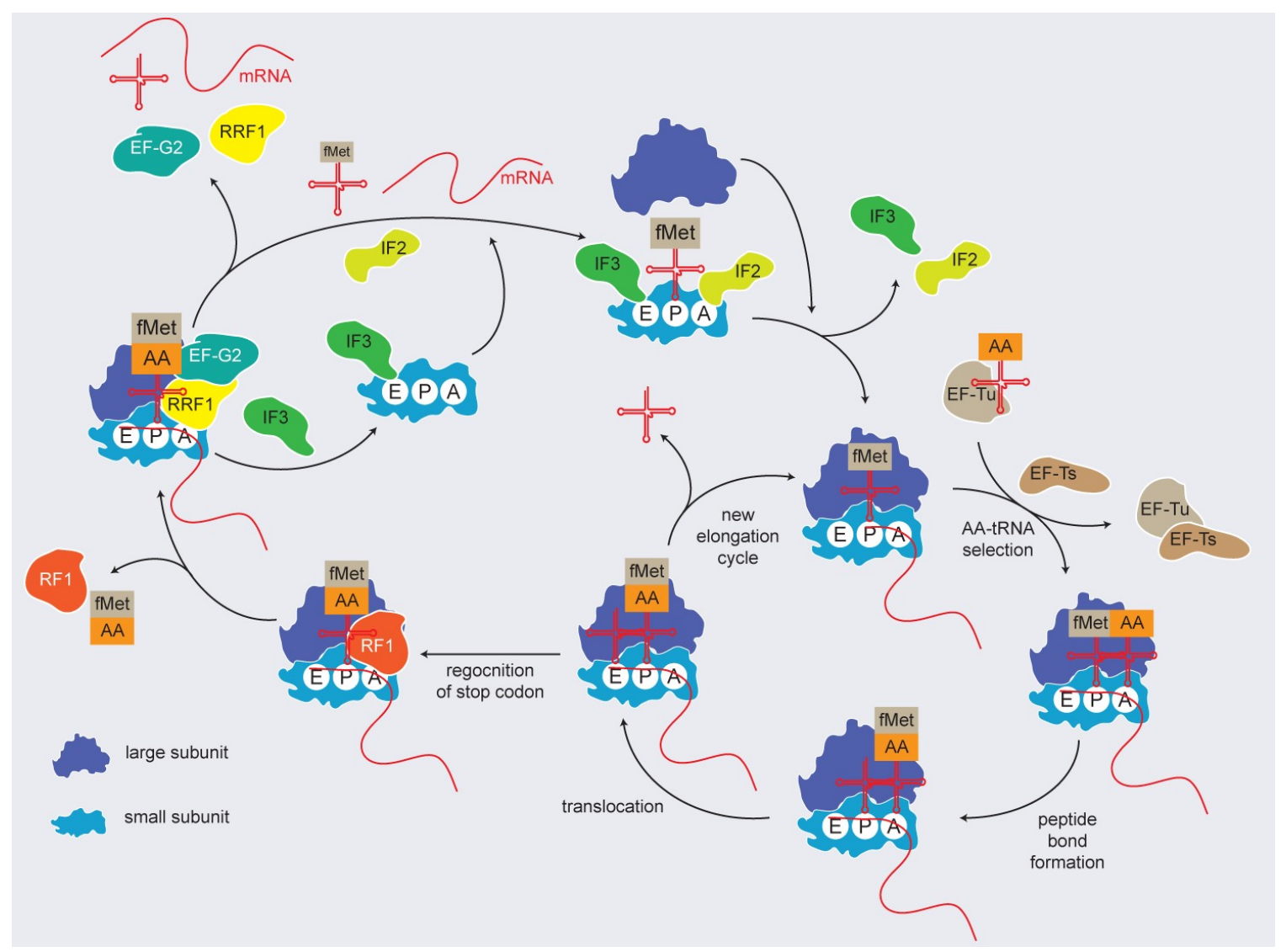

Figure 9 Mitochondrial translation cycle. Simplified depiction of mitochondrial translation.E, P, A: tRNA binding sites. Modified after (Ott et al., 2015).

Besides the factors that facilitate the different steps of mitochondrial translation, another set of translation regulators exist, which coordinates the translation of specific proteins in mitochondria. Most of these proteins interact with the mitochondrial ribosome and with the inner membrane. In yeast, these translation regulators promote mitochondrial translation of their target mRNA via interaction with mostly the 5'UTR of mRNA. By promoting translation, these proteins play a key role in the regulation of mitochondrial translation(Dennerlein et al., 2017). Such control mechanisms are very important for the assembly of the OXPHOS complexes, because of the dual genetic origins of their subunits, which must be coordinated to form mature complexes. Evidence of crosstalk in both directions between mitochondria and nucleus was provided recently by ribosome profiling analysis (Couvillion et al., 2016) and the uncovering of the mitochondrial compromised protein import response (Weidberg and Amon, 2018). Despite being investigated thoroughly in yeast, there is a lack of information regarding human translation 
regulators and since human mitochondrial mRNA do not have 5' UTR regions, the translation regulatory mechanisms are expected to differ from that of yeast. Studies on the translation of COX1 have provided some insight into the regulation of mitochondrial translation in human.

\subsubsection{COX1 translation in human mitochondria}

The first translational regulator discovered in human mitochondria was TACO1, which is involved in the synthesis of COX1. It was shown by Weraarpachai et al. that depletion of TACO1 leads to the specific decrease of COX1 translation (Weraarpachai et al., 2009). Furthermore, a recent study verified that TACO1 binds the mRNA of COX1 specifically and interacts with the mitochondrial ribosome. The mRNA binding domain of TACO1 belongs to one of the three domains of the DUF28 family, a group of putative proteins with unknown function (Richman et al., 2016). Until now, TACO1 remains the only known translation regulator with mRNA interaction in human mitochondria.

Nevertheless, other proteins were characterized that play a role in COX1 translation; MITRAC12 and C12ORF62, which bear similarities to the yeast assembly factors Coa3 and Cox14 respectively. First characterized in Drosophila as an essential protein for the functionality of complex IV (Peralta et al., 2012), MITRAC12 was shown to play a role in human mitochondria for COX1 synthesis and stability, in addition to assembly of complex IV (Mick et al., 2012). MITRAC12 and newly synthesized COX1 were shown to appear in an early assembly intermediate of complex IV assembly, termed MITRAC (Mitochondrial Translation Regulation Assembly intermediate of Cytochrome $c$ oxidase). The study by Mick et al. also characterized C12ORF62, another MITRAC subunit, as a factor necessary for COX1 translation, which was first described by Weraarpachai et al. in the same year (Weraarpachai et al., 2012). In a recent study, Richter-Dennerlein et al. it was shown that the MITRAC complex is a dynamic complex with changing composition, which interacts with the translating mitochondrial ribosome during COX1 synthesis. C12ORF62 was characterized as the first known MITRAC subunit to interact 
with the COX1 translating ribosome and was shown to copurify with nascent chains of COX1. Those nascent chains could accumulate with C12ORF62 if COX1 translation is disturbed by puromycin(Richter-Dennerlein et al., 2016). Furthermore, depletion of C12ORF62 reduces COX1 synthesis, suggesting its involvement in a regulatory feedback loop for COX1 translation (Mick et al., 2012; Richter-Dennerlein et al., 2016). Therefore, C12ORF62 has a specific role in the regulation of COX1 translation and its assembly into complex IV.

\subsection{Biogenesis of the oxidative phosphorylation system}

Mitochondria possess a highly diverse proteome. To date, 1837 mitochondrial and mitochondrial associated proteins have been identified in human (Palmfeldt and Bross, 2017) and over 3300 (Morgenstern et al., 2017) in yeast. Many of these proteins do not act alone and are organized into large complexes to fulfil their function within the organelle (MICOS, pyruvate dehydrogenase, translocases). The biogenesis of the complexes of the mitochondrial oxidative phosphorylation system (OXPHOS) is a unique process. Unlike other protein complexes, which consist solely of cytosolic translated proteins, OXPHOS complexes consist of subunits translated within both the cytosol and the mitochondria. Therefore, the assembly of OXPHOS is a complex procedure, where protein synthesis and protein import/export to the inner mitochondrial membrane have to be coordinated (Figure 10). Protein transport into the different compartments of mitochondria is realized by several distinct machineries. Most of them serve the purpose of protein import from the cytosol. Mitochondrial synthesized proteins are exported from the matrix to the IM via a conserved pathway. Despite being studied in detail within yeast, not all elements of the translocation machineries are conserved in human, therefore, protein import systems are only partially understood for human mitochondria (Kang et al., 2017;Wiedemann and Pfanner, 2017). 


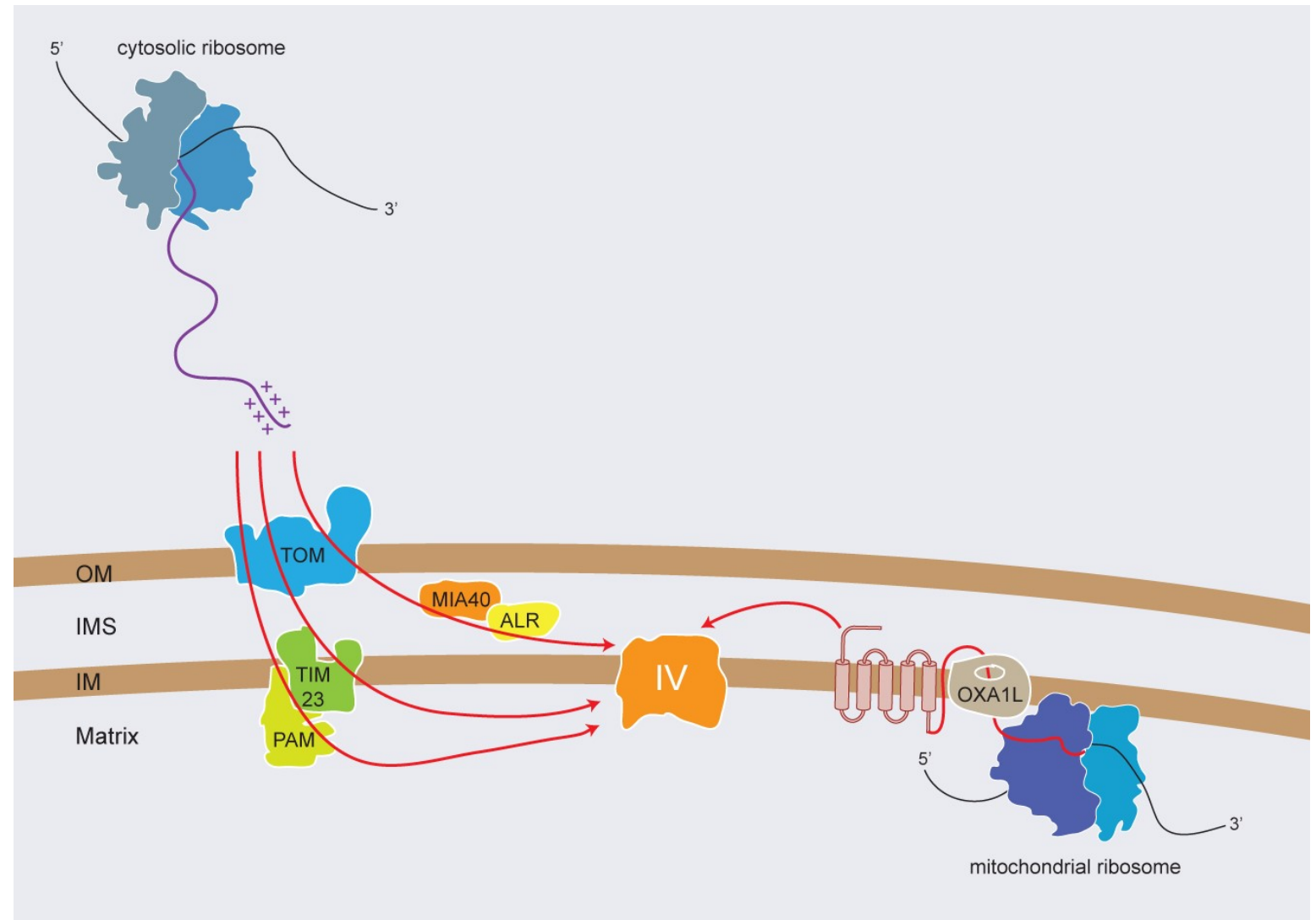

Figure 10 The mitochondrial complex IV contain subunits of dual origin. Complex IV like other OXHPOS components (I, III, V) have subunits of both cytosolic and mitochondrial translational origin. The insertion of mitochondrial-translated subunits depends on the OXA machinery, while the cytosolic synthesized subunits have to be imported by different pathways into mitochondria.

Cytosolic proteins destined for mitochondrial import first arrive at the translocase of the outer membrane (TOM). This translocase is responsible for the import of proteins into the IMS, where they are distributed further by a number of different transport systems, depending on their destination (Wiedemann and Pfanner, 2017). In both yeast and human, the TOM complex consists of the pore-forming protein TOM40 (Shiota et al., 2015), the receptor proteins: TOM20 (Abe et al., 2000), TOM22 (Yamano et al., 2008) and TOM70 (Brix et al., 2000), and three small TOM proteins: TOM5, TOM6, TOM7 (Kato and Mihara, 2008), which play a role in TOM assembly and stability (Shiota et al., 2015). In human mitochondria, hydrophobic precursor proteins interact with a number of chaperones including; HSP9O (Altieri, 2012), HSC70 (Zara et al., 2009), AIP (Shiota et al., 2015) and TOM34 (Chewawiwat et al., 1999), prior to their import through the TOM complex. 
The most well investigated import mechanism, which is also used by most of the OXPHOS subunits, is the TIM23-mediated presequence pathway to the inner mitochondrial membrane and the matrix. The presequence pathway covers the import of approximately $60 \%$ of proteins destined for the mitochondria (Vögtle et al., 2009). Presequence pathway substrates are characterized by a positively charged presequence, usually around 15 to 50 amino acids in length, which forms an amphipathic $\alpha$-helix with a positively charged and hydrophobic face (Roise et al., 1986). The presequence helix is recognised during protein import by receptors and components of the TOM, TIM23 and PAM machinery andthe membrane potential $(\Delta \Psi)$ facilitate the import of proteins with these positively charged presequences(Schulz et al., 2015).

The dynamic complex responsible for protein import into the mitochondrial inner membrane and the mitochondrial matrix is the Translocase of the Inner Membrane 23 (TIM23) complex. The yeast core complex consists of the presequence receptor, Tim50, which interacts with the protein precursors coming from the TOM complex (Schulz et al., 2011); the channel forming protein Tim23 (Denkert et al., 2017); the regulatory subunit Tim21 (Chacinska et al., 2005; van der Laan et al., 2006); and Tim17, which stabilizes the structure of the TIM23 complex (Matta et al., 2017). In human, the core components of TIM23 are largely conserved, although there are some differences. TIM17 exist in 2 isoforms: TIM17A and TIM17B. Despite both being expressed ubiquitously, TIM17A is rapidly degraded during stress conditions, whereas TIM17B has a more stable role in protein import (Rainbolt et al., 2013;Sinha et al., 2014).

Depending on the target of the substrate, the core TIM23 complex can associate with other proteins or complexes. Precursors of IM proteins contain a hydrophobic sorting signal (Botelho et al., 2011), which is supposed to be recognized by the small membrane protein assisting with their sorting, Mgr2 (leva et al., 2014). The release of those precursor proteins into the IM is driven by $\Delta \Psi$ (van der Laan et al., 2007), which is enhanced by the recruitment of complex III-IV supercomplexes by Tim21 in yeast (Wiedemann et al., 2007)(van der Laan et al., 2006). In human mitochondria, the mechanism of the Mrg2 homologue, ROMO1 (Žárský and Doležal, 2016), is 
enigmatic and TIM21 instead fulfils a shuttle function and is involved in transporting imported proteins to their assembly intermediates (Mick et al., 2012).

The $\Delta \Psi$ is not enough to drive protein import into the mitochondrial matrix alone. For this process, the core TIM23 complex associates with the presequence translocase-associated motor (PAM), whose function is evolutionally conserved between yeast and human mitochondria (Kang et al., 2017). The PAM complex consists of an ATP consuming chaperone as its core (mtHsp70 in yeast (Horst et al., 1997), mortalin in human (Yaguchi et al., 2007)) and catalyses the import of precursor proteins into the mitochondrial matrix.

Besides the presequence pathway, other import systems exist that suit the specific needs of substrates and target them to the appropriate mitochondrial compartment. The sorting and assembly machinery (SAM) serves to insert $\beta$ barrel proteins into the mitochondrial outer membrane (Klein et al., 2012) after import by the TOM complex (Jores et al., 2016). The mitochondrial inter membrane space assembly machinery (MIA), with its core enzymes, Mia40 (human: hMIA40) and Erv1 (human: ALR), specifically traps proteins with characteristic cysteine motifs, like $\mathrm{CX} 3 \mathrm{C}$ and $\mathrm{CX} 9 \mathrm{C}$,destined for the inner membrane space. Mia40interacts with them by the formation of disulfide bonds and catalyses the intramolecular disulfide bond formation of the target protein (Stojanovski et al., 2012). The carrier pathway, comprising of the TIM22 complex, imports hydrophobic, multitransmembrane domain proteins into the inner membrane. Substrates include components of different TIM machineries (Tim23, Tim22, Tim17) and mitochondrial carrier proteins(Rehling et al., 2004;Kang et al., 2016;Callegari et al., 2016).

Another crucial system, which is responsible for the membrane insertion of mitochondrial translated proteins, is the oxidase assembly (OXA) translocase. The main component of this system is Oxa1, a protein highly conserved from its bacterial ancestor, the YidC protein insertase, and was shown to export the mitochondrial translated proteins into the inner membrane in yeast (Hell et al., 2001). Furthermore, Oxa1 is involved in "conservative sorting" processes, where proteins imported by the presequence pathway into the mitochondrial matrix are re-exported into the inner mitochondrial membrane (Park et al., 
2013). The human homologueto Oxa1 is OXA1L and was shown to be able to substitute for Oxa1 in yeast (Bonnefoy et al., 1994). Nevertheless, it was reported byStiburek et al., that a knockdown of OXA1L only affect complex I and V, while complex III and IV are not affected (Stiburek et al., 2007). Hence the in detail function of OXA1L in human mitochondria needs to be further investigated.

\subsubsection{Biogenesis of the NADH Coenzyme $Q$ oxidoreductase}

The NADH Coenzyme Q oxidoreductase, also called mitochondrial complex I, is the largest of all OXPHOS complexes, with a size over $1 \mathrm{MDa}$. Complex I is the entry site of electrons provided by the citric cycle in the form of NADH (Wikström, 1984). The complex catalyzes the transfer of 2 electrons from $\mathrm{NADH}$ to ubiquinone and uses the chemical energy from that process to relocate 4 protons from the IMS to the mitochondrial matrix (Galkin et al., 1999). The structure of complex I (Figure 11)has an L-shaped architecture, which can be divided into a membrane arm and a peripheral arm (Agip et al., 2018;Fiedorczuk et al., 2016). The iron sulphur clusters are localized in the peripheral arm, which consists of the $N$ and $Q$ module (Agip et al., 2018; Fiedorczuk et al., 2016). While the $\mathrm{N}$ module is responsible for oxidising $\mathrm{NADH}$, the $\mathrm{Q}$ module handles the transfer of electrons to ubiquinone (Wirth et al., 2016). The reaction in the matrix arm induces conformational changes in the membrane arm, causing the translocation of protons via a still enigmatic mechanism. It has been proposed that the lateral a-helix of ND5, which lies planar to the membrane and reaches close to the ubiquinone binding site, plays a role in coupling the reduction of ubiquinone with the conformational change (Angerer et al., 2011). The membrane arm, consisting of the $P_{P}$ and the $\mathrm{P}_{\mathrm{D}}$ module, incorporates 4 proton channels, which consist of mitochondrial-translated subunits (Fiedorczuk et al., 2016). Mammalian complex I is composed of 45 subunits, 14 of which are core subunits, which are essential for the catalytic function and highly conserved from their bacterial ancestors. The accessory subunits of complex I bind peripherally to the conserved core in a cage-like structure (Sazanov et al., 2000). All the 
mitochondrial translated components of complex I belong to the group of core subunits (Vinothkumar et al., 2014;FiedorczukWirth et al., 2016).

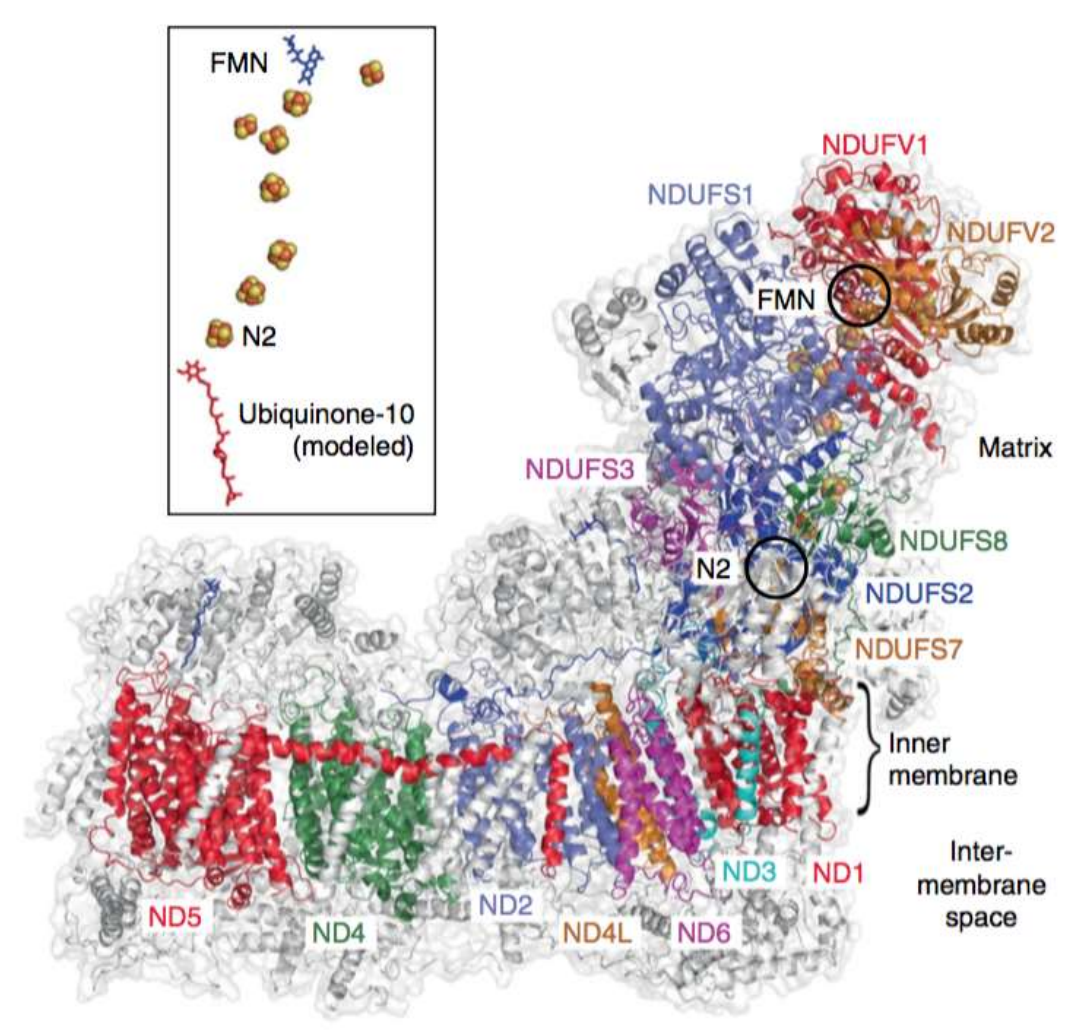

Figure 11Structure of the mouse mitochondrial complex I. Taken from (Agip et al., 2018). The positions of the Fe-S clusters are highlighted separately.

The assembly of the mitochondrial complex I was characterized as a modular process by several groups (Stroud et al., 2016; Perales-Clemente et al., 2010; Guerrero-Castillo et al., 2017). Different modules of complex I assemble first into intermediate complexes, which are then further assembled into the mature complex. While the $\mathrm{N}$ and $\mathrm{Q}$ module assemble as distinct intermediates, the $P_{P}$ module is divided into two submodules; $P_{P}-a$ (ND1module) and $\mathrm{P}_{\mathrm{P}}-\mathrm{b}$ (ND2-module) and the $\mathrm{P}_{\mathrm{D}}$ module is divided into $\mathrm{P}_{\mathrm{D}}-\mathrm{a}$ (ND4-module) and $\mathrm{P}_{\mathrm{D}-\mathrm{b}}$ (ND5-module). By using mass spectrometry analytics, in combination with blue native PAGE, recent studies have provided an insight into the hierarchical order of human complex I assembly (Stroud et al., 2016; Guerrero-Castillo et al., 2017). The current model states that the modules: $N, Q, P_{P}-b, P_{D}-a$ and $P_{D}-b$ assemble independently of each other, while the $P_{P}$-a module assembles onto the mature $Q$ module. 
The $\mathrm{N}$ module assembles around the three core subunits; NDUFS1, NDUFV1 and NDUFV2 (Berrisford and Sazanov, 2009). Even though there is no experimental evidence of a stable assembly intermediate containing all $\mathrm{N}$ module subunits, an intermediate complex with the three core subunits of the $\mathrm{N}$ module was characterized in a knockdown of TIMMDC1, an assembly factor of the $P_{P}-a$ module (Guarani et al., 2014). The assembly of the $N$ module is possibly assisted by NDUFAF2 and NUBPL. NDUFAF2 occurred from a duplication event of the accessory $\mathrm{N}$ module subunit NDUFA12 (Ogilvie et al., 2005), and was shown to be able to interact with the $\mathrm{N}$ module at the position of NDUFA12, even though it cannot replace NDUFA12 to form a functional complex (Ostergaard et al., 2011). NDUFAF2 is not present in mature complex I, but it can interact with a stable assembly intermediate of complex I and with all modules except for the $\mathrm{N}$ module (Lazarou et al., 2007; Vogel et al., 2007d). NUBPL is a putative assembly factor of the $\mathrm{N}$ module, which is believed to be involved in the incorporation of Fe-S clusters (Sheftel et al., 2009) and acts in the early assembly of the $\mathrm{N}$ module (Kevelam et al., 2013). NUBPL harbours a conserved cysteine motif (CXXC), enabling the binding of Fe-S clusters (Bych et al., 2008). Patients with mutations in the NUBPL gene were shown to have complex I deficiency (Sheftel et al., 2009). The $Q$ module is formed around four core subunits; NDUFS2, NDUFS3, NDUFS7 and NDUFS8 (Brandt, 2006). In a first assembly step, NDUFS2 and NDUFS3 form a complex with the accessory subunit NDUFA5, followed by the addition of the Fe-S cluster containing proteins, NDUFS7 and NDUFS8 (Guerrero-Castillo et al., 2017). The assembly factors NDUFAF3 and NDUFAF4 then join the still soluble assembly intermediate (Saada et al., 2009; Guerrero-Castillo et al., 2017). Two other assembly factors acting in the assembly of the $Q$ module are NDUFAF5 and NDUFAF7. NDUFAF5 mutations were discovered in patients with complex I deficiency (Sugiana et al., 2008) and the assembly factor was characterized as an arginine hydroxylase, which adds a hydroxyl group at Arg-73 of the accessory $Q$ module subunit, NDUFS7 (Rhein et al., 2016). NDUFAF7 interacts with the core subunit of the Q module, NDUFS2 (Carilla-Latorre et al., 2010), and is a class II methlytransferase, which is required for dimethylation at Arg-85 of 
NDUFS2 (Rhein et al., 2013). Furthermore, it has been proposed that NUBPL plays a role in the incorporation of Fe-S clusters.

The assembly of the $\mathrm{P}_{\mathrm{P}}$ module is divided into the assembly of $\mathrm{P}_{\mathrm{P}}-\mathrm{a}$ and $\mathrm{P}_{\mathrm{P}}-\mathrm{b}$. Interestingly, these two subassembly intermediates do not join directly after their assembly but associate with other modules(Guerrero-Castillo et al., 2017). $P_{P}-a$, together with the core subunit ND1, assembles onto the mature $Q$ module and serves as an anchor to recruit it to the inner membrane(Sánchez-Caballero et al., 2016). This process is assisted by the $Q$ module assembly factors, NDUFAF3 and NDUFAF4 (Andrews et al., 2013), and by the $P_{P}-a$ module assembly factor of the TIM17/22/23 family, TMMCD1(Guarani et al., 2014), which are believed to prevent degradation of the complex by proteases(Zurita Rendon and Shoubridge, 2012). Furthermore, NDUFAF5, NDUFAF6 and NDUFAF7 were reported to have an effect on the translation of ND1.It was observed in cells with NDUFAF5 and NDUFAF6 mutations, or NDUFAF7 depletion, that the translation of ND1 is reduced(Sugiana et al., 2008;Zurita Rendón et al., 2014). Nevertheless, a direct interaction between NDUFAF5 and NDUFAF6 with newly synthesized ND1 or ND1 mRNA was never detected. This brings into question their role as translation regulators of ND1.

Unlike the $\mathrm{P}_{\mathrm{P}-\mathrm{a}}$ module, the $\mathrm{P}_{\mathrm{P}-\mathrm{b}}$ module, with its core subunit ND2, assembles independently of other subcomplexes (Guerrero-Castillo et al., 2017). It was shown by Guerrero Castillo et al. that the earliest $P_{P}-b$ module assembly intermediate consist of the core subunits ND2, the accessory subunits, NDUFC1 and NDUFC2, and the assembly factors ACAD9, MITRAC15 (COA1), ECSIT and NDUFAF1(Guerrero-Castillo et al., 2017)Later, the core subunit ND3 joins the complex, with the assembly factors TMEM126B and TMEM186, followed by two additional core subunits, ND4L and ND6. It has also been reported that the $P_{\mathrm{P}}-\mathrm{b}$ module assembly factors, ACAD9, ECSIT, NDUFAF1, TMEM126B and the $P_{\mathrm{P}}-\mathrm{a}$ module assembly factor, TMMCD1, form a complex before interacting with complex I subunits (Giachin et al., 2016). The complex is called the mitochondrial complex I assembly (MCIA) complex.

NDUFAF1 was shown to interact with complex I subunits of both mitochondrial and cellular translational origin (Dunning et al., 2007) and a 
depletion of NDUFAF1 led to a decrease in complex I activity (Vogel et al., 2005). Therefore, NDUFAF1 is believed to carry out chaperone functions, while interacting transiently with proteins of the $P_{P}-b$ module, however, the mechanism is still enigmatic (Vogel et al., 2007c). ECSIT (Evolutionarily conserved signalling intermediate in Toll pathway) was first identified as a cytosolic adaptor protein, participating in the Toll pathway (Kopp et al., 1999). It was later shown to posses an N-terminal MTS of 48 amino acids that can direct the protein to mitochondria (Vogel et al., 2007b). Furthermore, cells with a knockout of ECSIT have reduced NDUFAF1 levels, which lead to a decrease in complex I activity (Vogel et al., 2007b). Interestingly, even though both NDUFAF1 and ECSIT posses the putative RNA binding motifs, RNA recognition motif $(R R M)$ and pentatricopeptide repeat motif (PPR) respectively, no RNA interaction was ever detected for those proteins (Marchler-Bauer et al., 2014;Giachin et al., 2016).

Similar to ECSIT, ACAD9 (Acyl-CoA dehydrogenase 9) was also first believed to carry out a function unrelated to OXPHOS assembly, because it belongs to the ACAD family, a group of flavoenzymes that catalyze the rate-limiting step of fatty acid $\beta$-oxidation (He et al., 2007). Later, it was shown that a knockdown of ACAD9 impairs the assembly of complex I and also causes reduced levels of NDUFAF1 and ECSIT (Nouws et al., 2010). Interestingly, it was shown that a catalytically inactive mutant of ACAD9 was able to perform its function as an assembly factor (Nouws et al., 2013;Schiff et al., 2015). This information suggests that ACAD9 might fulfil two distinct roles as complex I assembly factor and in fatty acid $\beta$-oxidation.

TMEM126B belongs to the TIM17/22/23 family, like the $Q$ module assembly factor, TMMCD1 (Andrews et al., 2013). Being a membrane protein, it was suggested that TMEM126B coordinates the membrane association of the soluble proteins in the MCIA complex, which is supported by the observation that NDUFAF1, ECSIT, and ACAD9 are not recruited to the mitochondria IM if TMEM126B is depleted (Vartak et al., 2014). The connection between MITRAC15 and complex I was first reported by Mick et al., where MITRAC15 was described as an assembly factor acting in the assembly pathways of both complex I and IV (Mick et al., 2012). In a more recent publication by Guerrero Castillo et al., MITRAC15 was shown to be one of the first subunits of the $\mathrm{P}_{\mathrm{P}}$ 
b module(Guerrero-Castillo et al., 2017). However, its function still needs to be investigated.

The $P_{D}$ module also assembles in two distinct intermediates $\left(P_{D}-a, P_{D}-b\right)$ (Guerrero-Castillo et al., 2017). In the case of the $P_{D}-a$ module, assembly starts with the nuclear encoded accessory subunits NDUFB5, NDUFB10 andNDUFB11, which form the first assembly intermediate. NDUFB6 then joins in a following step (Guarani et al., 2014). NDUFB1 and the mitochondrialsynthesized core subunit, ND4, are then added in a final step (GuerreroCastillo et al., 2017). The $P_{D}$-a assembly intermediate coassociates with three assembly factors,FOXRED1, ATP5SL and DMAC1,all of which have uncharacterized functions(Guerrero-Castillo et al., 2017). While it is suggested that FOXRED1 acts in the late assembly of complex I (Formosa et al., 2015), ATP5SL and DMAC1 were reported to interact with newly synthesized, unassembled, ND5, as well as OXA1L, suggesting a role as translation regulators(Stroud et al., 2016). For the $P_{D}-b$ module, it's assembly still remains enigmatic and no subassembly intermediates have been detected.

The first assembly steps of complex I lead to the formation of 5 distinct intermediates ( $\mathrm{N}$ module, $\mathrm{Q}-\mathrm{P}_{\mathrm{P}}-\mathrm{a}$ module, $\mathrm{P}_{\mathrm{P}-\mathrm{b}}$ module, $\mathrm{P}_{\mathrm{D}}-\mathrm{a}$ and $\mathrm{P}_{\mathrm{D}}-\mathrm{b}$ module), which all have to be joined together to form the mature complex I. The traditional model proposes that the first step is the assembly of the $P_{P}-b$

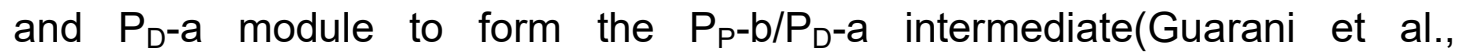
2014; Perales-Clemente et al., 2010). During this assembly step, NDUFS5, NDUFA10 and NDUFB4 are incorporated into the $\mathrm{P}_{\mathrm{P}}-\mathrm{b} / \mathrm{P}_{\mathrm{D}}-\mathrm{a}$ intermediate(Guerrero-Castillo et al., 2017). In the following step, both the Q$\mathrm{P}_{\mathrm{P}-\mathrm{a}}$ module and the $\mathrm{P}_{\mathrm{D}}-\mathrm{b}$ module assembles onto the $\mathrm{P}_{\mathrm{P}}-\mathrm{b} / \mathrm{P}_{\mathrm{D}}-\mathrm{a}$ intermediate, forming the Q/P assembly intermediate(Vogel et al., 2007a), which is possibly accompanied by the incorporation of NDUFA9and the assembly factor NDUFAF2(Sánchez-Caballero et al., 2016). Nevertheless, it was shown in the mass spectrometry analysis of complex I assembly intermediates on BN gels by Guerrero Castillo et al., that the $\mathrm{P}_{\mathrm{P}}-\mathrm{b}$ module can assembly with the $\mathrm{Q}-\mathrm{P}_{\mathrm{P}}$-a module directly, while the $\mathrm{P}_{\mathrm{D}}$-a module assembles onto that intermediate (Guerrero-Castillo et al., 2017). This observation showed that the assembly of complex $\mathrm{I}$ is more dynamic than assumed.The 
final steps include the addition of the $\mathrm{N}$ module, which is assisted by NDUFAF2 and the removal of the assembly factors(Schlehe et al., 2013). During the final assembly steps, NDUFS6 and the NDUFAF2 homologue, NDUFA12 are added to the complex, forming a mature complex I(Kmita et al., 2015).Figure 12 summarizes the modular assembly of the mitochondrial complex I. 


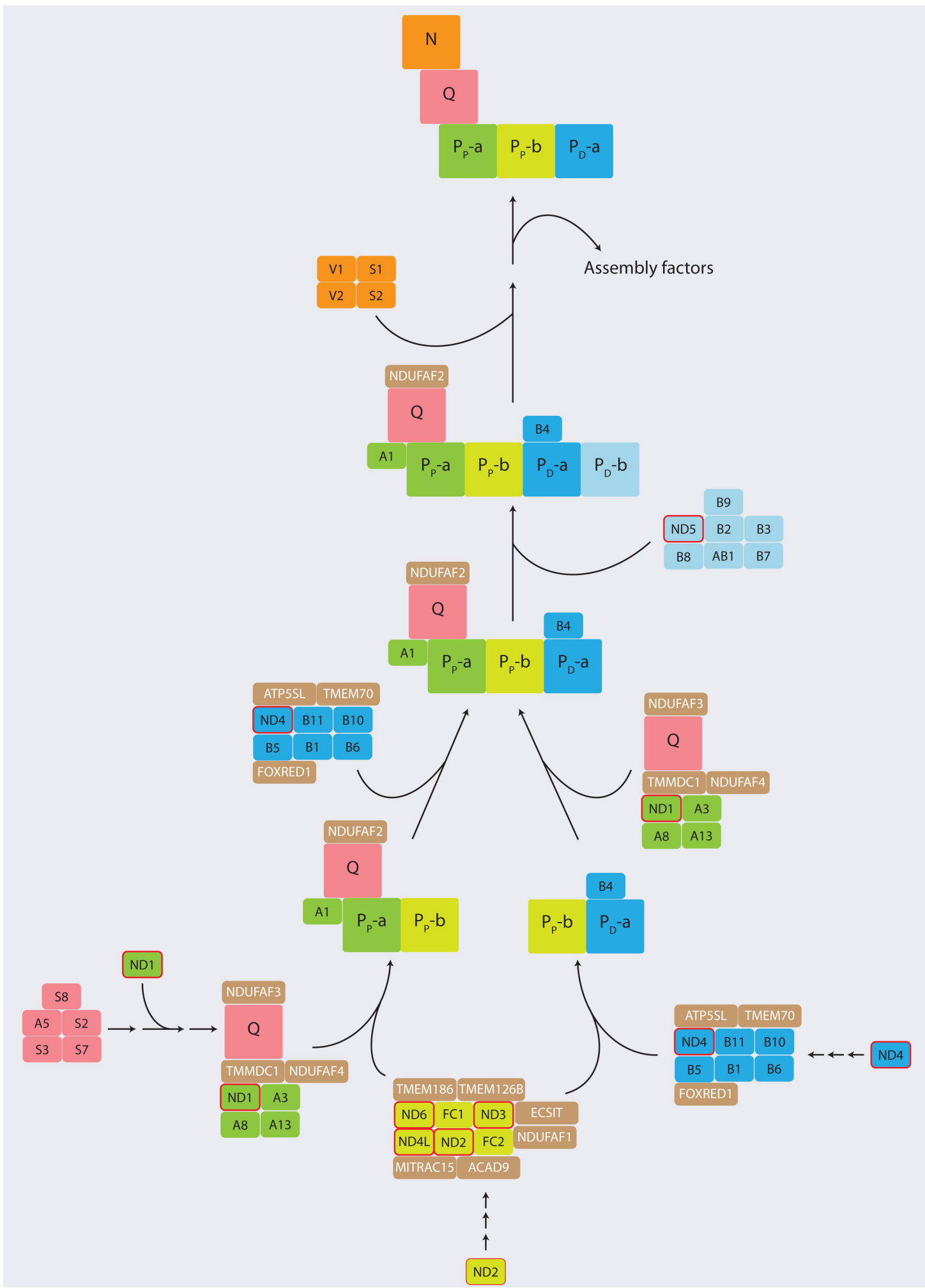

Figure 12 Modular assembly of the mitochondrial complex I. Mitochondrial translated subunits are marked with a red border. "NDUF" was left out of the name of complex I subunits. The assembly factors of complex I are shown in brown and the names were not shortened. Modified after (Guerrero-Castillo et al., 2017). 


\subsubsection{Biogenesis of the cytochrome $c$ oxidase}

The cytochrome $c$ oxidase, or mitochondrial complex IV, is the last enzymatic complex within the electron transport chain. After cytochrome $c$ is reduced by complex III, complex IV oxidize cytochrome $c$ and transfers the electrons to oxygen, reducing it to water(Wikström and Sharma, 2018). For the reduction of each $\mathrm{O}_{2}$ molecule, four electrons has to be provided by two molecules of reduced cytochrome $\mathrm{c}$, while four protons are consumed for the reaction of $\mathrm{O}_{2}$ to twomolecules of $\mathrm{H}_{2} \mathrm{O}$. During this process, four additional protons are transferred from the mitochondrial matrix into the IMS (Wikström et al., 2017). The cytochrome $c$ oxidase belongs to the Type A1 heme-copper oxidases, which are characterized by an active site (binuclear center / BNC)(Figure 13B), harbouring a heme $a_{3}$. The catalytic core of complex IV consists of three highly hydrophobic mitochondrial translated subunits, COX1, COX2 and COX3(Michel et al., 1998) (Figure 13A). Electrons provided by reduced cytochrome $c$ arrive first in the $\mathrm{Cu}_{\mathrm{A}}$ centre, localized in COX2(Brändén et al., 2002). The $\mathrm{Cu}_{A}$ centre then transfers the electrons to the heme a molecule in COX1, which then delivers it to the BNC(Kaila et al., 2010). The BNC consists of heme $\mathrm{a}_{3}$ and $\mathrm{a} \mathrm{Cu}_{\mathrm{B}}$ centre The $\mathrm{Cu}_{\mathrm{B}}$ centre is comprised of a copper ion with 3 histidine ligands and a tyrosine residue linked to one of the histidines(Wikström et al., 2017). The BNC transfers the electrons to $\mathrm{O}_{2}$, which is bound by heme $a_{3}$, and finishes the chemical reaction(Gorbikova et al., 2008). During this electron transfer, protons are transferred to the IMS by a proton pump with a proton-loading site, whose exact position could not be determined until now(Wikström and Sharma, 2018). Unlike COX1 or COX2, COX3 does not actively participate in the catalytic process but stabilizes the other two core subunits(Haltia et al., 1991). 
A

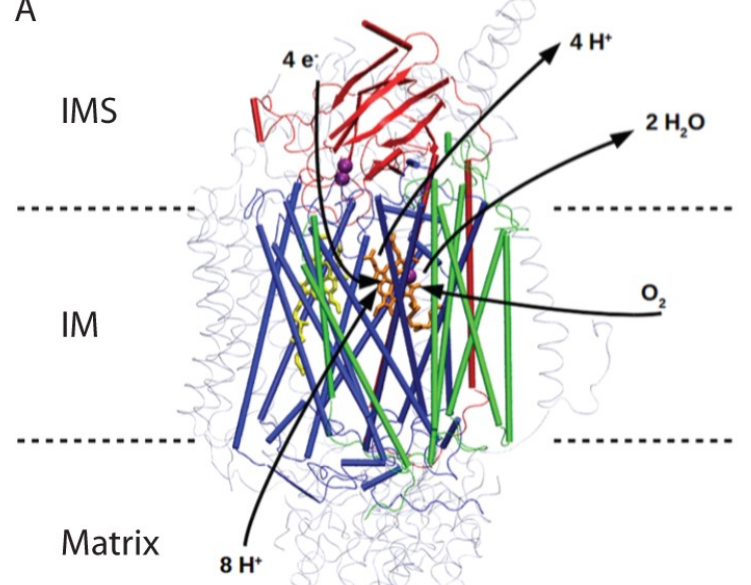

B

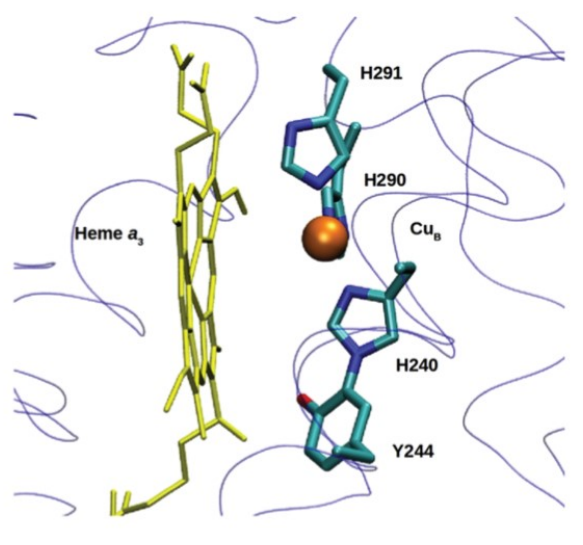

Figure 13 Structure of the cytochrome $c$ oxidase. A) Structure of the cytochrome $c$ oxidase. The mitochondrial translated core subunits $\operatorname{COX} 1, \operatorname{COX} 2$ and $\operatorname{COX} 3$ are highlightened and shown in blue, red and green respectively, while the cytosolic translated accessory subunits are shown in white. The chemical reaction catalysed by the complex is simplified. Taken from (Wikström et al., 2017). B) Binuclear center of complex IV. Taken from (Wikström and Sharma, 2018).

The assembly of the mitochondrial complex IV is a highly complex process, partially due to the specific cofactors needed in this complex. Three Core subunits and 11 accessory subunits are assembled by more than 30 known assembly factors(Timón-Gómez et al., 2018). Even though the assembly of the cytochrome $c$ oxidase was initially perceived to be linear (Wielburski and Nelson, 1983), recent studies in yeast and human mitochondrianow favour a modular process(Timón-Gómez et al., 2018;Vidoni et al., 2017;Aich et al., 2018). COX1 and COX2 where shown to be processed individually, before forming subassembly intermediates and joining in the formation of the mature complex IV(Vidoni et al., 2017). Nevertheless, processing of COX3 and the late stage assembly of complex IV still remains enigmatic.

\subsubsection{Processing and assembly of COX1}

\subsubsection{1.a COX1 translation and early assembly factors}

With 12 transmembrane domains and a size of $57 \mathrm{kDa}$, COX1 is the largest subunit of complex IV and contain three of its four metal centres (Michel et al., 1998). The synthesis of COX1 is regulated by three known factors; TACO1, which interacts with the mRNA of COX1 (Richman et al., 2016); C12ORF62, 
which is crucial for COX1 translation and association with the COX1 translating ribosome; and, to a lesser extent, MITRAC12 (Richter-Dennerlein et al., 2016), which also associates with the COX1 translating ribosome, but plays more of a role in stabilizing synthesized COX1 (Mick et al., 2012). The first assembly intermediate with which COX1 associates, was termed MITRAC (Mick et al., 2012). The early stages of MITRAC already begin to assemble during the synthesis of COX1, with C12ORF62 being the earliest identified MITRAC subunit, followed by MITRAC12 (Richter-Dennerlein et al., 2016). After the termination of COX1 translation and disassociation from the mitochondrial ribosome, MITRAC is visible in a range between 140 and 200 $\mathrm{kDa}$ on BN-PAGE and contains COX4I-1, one of the first cytosolic synthesized complex IV subunits to join the complex (Mick et al., 2012). Bourens et al. characterized the protein $\mathrm{CMC1}$, a twin $\mathrm{CX} 9 \mathrm{C}$ motif containing protein that acts in complex IV assembly and comigrates with MITRAC on BNPAGE. However, CMC1 does not influence the synthesis of COX1 (Bourens and Barrientos, 2017a). CMC1 interacts with COX1, MITRAC12 and C12ORF62, but not with COX4I-1 (Bourens and Barrientos, 2017a). This argues that $\mathrm{CMC} 1$ only associates with MITRAC for a short period before COX4I-1 joins the complex. Furthermore, Vidoni et al. (Vidoni et al., 2017) detected a low molecular weight complex consisting of COX4I-1, COX5A and HIG1A by SILAC analysis from a BN-PAGE of DDM solubilized mitoplasts. HIG1A was characterized as an assembly factor for complex IV and it was suggested that COX4I-1 and COX5A assemble from this complex into MITRAC, while losing HIG1A (Vidoni et al., 2017).

\subsubsection{1.b The TIM21 shuttle and late MITRAC}

The MITRAC complex also interacts with the TIM23 translocase subunit, TIM21. In yeast, TIM21 promotes the interaction of the TIM23 complex with the TOM machinery and counteracts its interaction with the PAM complex, However, TIM21 fulfils a different function in human mitochondria. Mick et al. showed that the association of TIM21 can shift either towards MITRAC, or to the TIM23 complex, depending on whether cellular or mitochondrial translation is inhibited respectively. Furthermore, TIM21 is needed for the assembly of cytosolic synthesized complex IV subunits into MITRAC (Mick et 
al., 2012).Based on these findings, a shuttle function was assigned to TIM21, implicating it in the transport of imported proteins from the translocase to MITRAC for further assembly. Additionally MITRAC15 was characterized as an assembly factor, which acts in both complex I and complex IV assembly and interacts with subunits of both complexes (Mick et al., 2012). In a more recent publication, MITRAC7 was characterized by Dennerlein at al. The interaction between MITRAC7 and COX1 was reduced in cells lacking complex IV (specifically the nuclear-encoded subunits COX4I-1 or COX6C), or TIM21, while the interaction between MITRAC12 and COX1 remained stable in those cells. Therefore, MITRAC7 was expected to act in the late stages of MITRAC (Dennerlein et al., 2015).Figure 14 summarized the present model of COX1 assembly in the MITRAC complex.

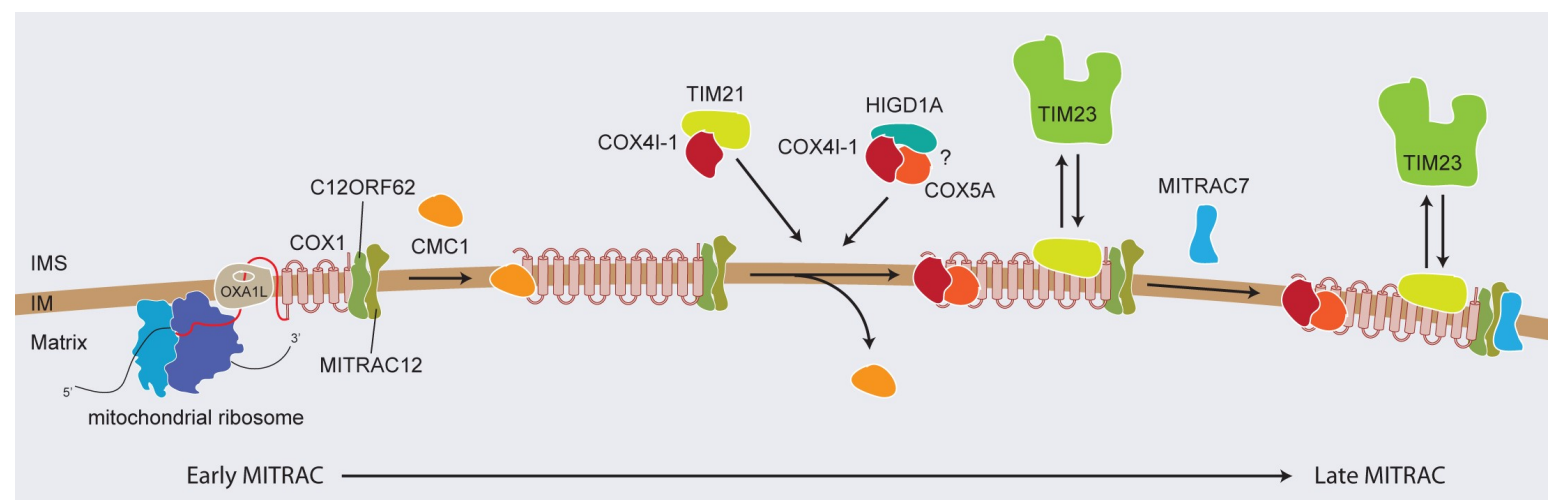

Figure 14 Assembly of the MITRAC complex. The MITRAC complex assembles during COX1 translation and coordinates the assembly of the COX1 module. TIM21 shuttles between TIM23 and MITRAC.

\subsubsection{1.c Heme insertion into COX1}

A crucial step in providing two heme A molecules for the heme $a$ and heme $a_{3}$ centres of COX1 is the biosynthesis of heme A from heme B. The conversion is performed by two enzymes, which are integrated in the inner mitochondrial membrane, COX10 and COX15 (Soto et al., 2012;Antonicka, 2003;Antonicka et al., 2003). While this process remains enigmatic for mitochondria in human, some information exists for the mechanism of heme $A$ insertion into Cox 1 in yeast. Cox10 (Khalimonchuk et al., 2012) and Cox15 (Swenson et al., 2016) were shown to oligomerize in yeast mitochondria and the oligomerization of Cox15 was proven to be crucial for both heme $A$ biogenesis and insertion into Cox1 (Swenson et al., 2016). Cox15 oligomerization in yeast is promoted by 
its interaction with Pet117 (Taylor et al., 2017). PET117 in human was characterized as a late assembly factor for complex IV (Vidoni et al., 2017), but its role in heme $A$ insertion has yet to be investigated. In human mitochondria, heme $A$ insertion is suggested to take place in the later steps of complex IV assembly, since the formation of MITRAC was shown to be independent of COX10 (Bourens and Barrientos, 2017a).

\subsubsection{1.d Copper insertion into COX1}

Unlike the maturation of the heme centers in COX1, more information is available on copper insertion into the $\mathrm{Cu}_{B}$ site. For both the $\mathrm{Cu}_{\mathrm{B}}$ site in COX1, as well as the $\mathrm{Cu}_{\mathrm{A}}$ site in $\mathrm{COX} 2$, copper ions are provided by COX17(Oswald et al., 2009). COX17 is a conserved protein localized in the mitochondrial IMS and contains two hydrophobic CX9C motifs(Glerum et al., 1996). Copper is bound by $\mathrm{COX} 17$ with a $\mathrm{CCxC} \mathrm{Cu(I)} \mathrm{binding} \mathrm{motif} \mathrm{and} \mathrm{even}$ though COX17 is able to bind multipleCu(I) ions in its fully reduced state(Beers et al., 1997), it is believed to only transport one $\mathrm{Cu}(\mathrm{I})$ ion at a time(Banci et al., 2008). For copper delivery to the $\mathrm{Cu}_{\mathrm{B}}$ site of $\mathrm{COX} 1$, the $\mathrm{Cu}(\mathrm{I})$ ion provided by COX17 is received by the copper chaperone COX11(Hiser et al., 2000). COX11 consists of a C-terminal soluble domain located in the IMS, which is coupled to an a-helical membrane anchor by a flexible linker of 15 amino acids(Banci et al., 2004). The soluble domain of COX11 harbours a conserved CFCF motif and it was shown in yeast that both cysteines are important for copper binding and that COX11 forms a dimer with its soluble domain, in order to bind one $\mathrm{Cu}(\mathrm{I})$ ion with each monomer(Hüttemann et al., 2001; Carr et al., 2002). The current model in yeast states that the soluble domains of the COX11 dimer are positioned above COX1 and the $\mathrm{Cu}(\mathrm{I})$ ion is transferred to the histidines of the $\mathrm{Cu}_{B}$ site, with the help of a reduced Cys111 in COX1(van Dijk et al., 2007; Thompson et al., 2010). This model is supported by the essentiality of COX19 for complex IV assembly, which is a twin CX9C protein that keeps Cys-111 in a reduced state(Bode et al., 2015). In human, COX11 and its ability to bind copper are well conserved(Banci et al., 2004). However, in COX19, the highly conserved dipeptide Tyr-Leu, which is crucial for its structure in yeast, is exchanged by Phe-Met in human mitochondria(Bode et al., 2015). Still, Phe-Met provides the hydrophobicity 
and steric hindrance, which was a crucial feature in Tyr-Leu. Therefore, it is likely that $\mathrm{Cu}_{\mathrm{B}}$ maturation is similar in yeast and human.

\subsubsection{Processing and assembly of COX2}

\subsubsection{2.a COX2 membrane insertion and early assembly factors}

COX2 consists of a C-terminal globular domain localized in the IMS, which harbours the $\mathrm{Cu}_{\mathrm{A}}$ centre, with two copper ions, and two $\mathrm{N}$-terminal transmembrane helices anchored in the IM (Soto et al., 2012). In Yeast, Cox2 translation is assisted by the translation factor Pet111 (Green-Willms et al., 2001), which promotes the synthesis of the precursor protein, pCox2 (Lorenzi et al., 2016). The first $\mathrm{N}$-terminal transmembrane helix is inserted into the inner mitochondrial membrane by Oxa1 (Bonnefoy et al., 2009), while Mba1 (the yeast homologue of $\mathrm{mL} 45$ ) mediates the membrane association of the mitochondrial ribosome during translation (Lorenzi et al., 2016). Mba1 then shuttles pCox2 to Cox 20 , a Cox 2 chaperone whose interaction with pCox2 promotes the cleavage of the $\mathrm{N}$-terminal extension of pCox2 by the inner membrane peptidase complex that comprises of Imp1, Imp2 and Som1 (Jan et al., 2000). After N-terminal cleavage, the membrane proteins Cox18, Mss2 and Pnt1 promote the translocation of the C-terminal globular domain and the insertion of the second transmembrane helix into the IM (Bonnefoy et al., 2009;Saracco and Fox, 2002). Interestingly, Cox18 resembles the Oxa1 family of insertases, but its functionality is specific for Cox2 (Bonnefoy et al., 2009). In human, the there is no N-terminal processing of COX2, also the human Oxa1 homologue, OXA1L, is not mandatory for the assembly of complexes III and IV (Stiburek et al., 2007), suggesting that other proteins can functionally substitute this translocase. Nevertheless, COX20 and COX18 are conserved from yeast to human and were shown to be crucial for the stability of COX2 and therefore further assembly of complex IV (Bourens et al., 2014;Bourens and Barrientos, 2017b). Additionally, human COX18 is responsible for the translocation of the C-terminal soluble domain of COX2 to the IMS, like its yeast homologue (Bourens and Barrientos, 2017b), suggesting some similarities for COX2 biogenesis in general. Nevertheless, 
Lorenzi et al. characterized the human COX2 associated assembly factor TMEM177, which is not present in yeast. TMEM177 interact with COX2, SCO1, SCO2 and COX2, but not with COX18 and therefore associate with COX2 in its late stage maturation (Lorenzi et al., 2018). These data indict, that the maturation and assembly of COX2 into the mature mitochondrial complex IV has changed from yeast to human.

\subsubsection{2.b Copper insertion into COX2}

The $\mathrm{Cu}_{\mathrm{A}}$ centre of $\mathrm{COX} 2$ consists of two copper ions, bridged by the thiol side chains of two cysteine residues (Wikström et al., 2017). The reduced states of the thiol groups are crucial, since copper binding can be prevented by their oxidation (Timón-Gómez et al., 2018). Copper insertion into COX2 is coordinated by two assembly factors, SCO1 and SCO2 (Abriata et al., 2008). These copper chaperones posses a globular domain located in the mitochondrial IMS, which is tethered to the IM with a single $\alpha$-helix (Banci et al., 2007a). They can bind one copper ion in both $\mathrm{Cu}(\mathrm{I})$ and $\mathrm{Cu}(\mathrm{II})$ oxidative state, with a CXXXC thioredoxin like motif (Banci et al., 2007a), which also allows them to reduce the cysteine residues in the $\mathrm{Cu}_{\mathrm{A}}$ centre(Banci et al., 2007b). Even though the two SCO proteins are closely related, they are both essential for $\mathrm{Cu}_{\mathrm{A}}$ maturation and they cannot compensate for each other's functions(Leary et al., 2009). The current model states that the SCO1 and SCO2 transfer the copper ions to the $\mathrm{Cu}_{\mathrm{A}}$ centre, while reducing the cysteine residues(Timón-Gómez et al., 2018). After the copper transfer SCO1 is present in its reduced state and SCO2 in its oxidized state. SCO2 would then be reduced by SCO1 and both chaperones will be loaded with copper from COX17, preparing them for a new round of $\mathrm{Cu}_{\mathrm{A}}$ maturation(Banci et al., 2008). In addition to copper transfer, COX17 also performs the reduction of oxidized SCO1 (Leary et al., 2009). Another assembly factor that assists in COX2 maturation in human is COA6, a soluble protein in the IMS. Pacheu et al. showed that COA6 affects the stability of COX2 and specifically interacts with the copper chaperone, $\mathrm{SCO} 2$, suggesting a role in $\mathrm{Cu}_{\mathrm{A}}$ maturation(PacheuGrau et al., 2015).Figure 15 summarizes the events during COX2 maturation. 


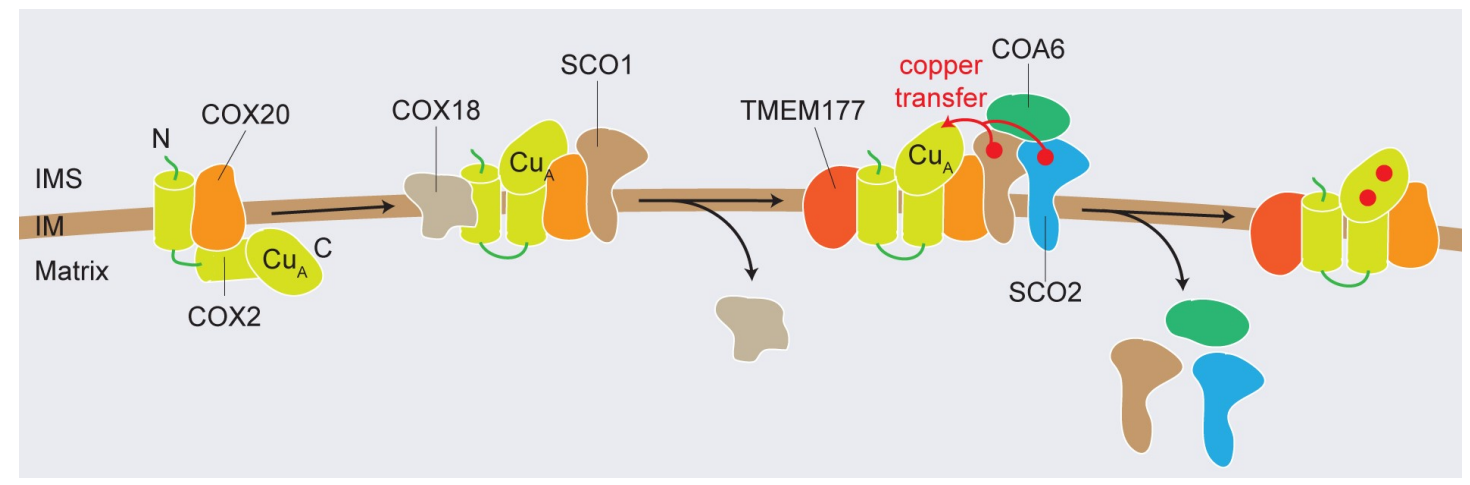

Figure 15 Maturation of COX2.

\subsubsection{Late stage assembly of the cytochrome $c$ oxidase}

Very little is currently known about the later stages of complex IV assembly. A differentiation between late and early stage complex IV subunits in human was first attempted by Lazarou et al. (Lazarou et al., 2009), who characterized COX4I-1 as an early subunit and COX6A and COX7A as late subunits of the cytochrome $c$ oxidase. Recently, Vidoni et al. (Vidoni et al., 2017) characterized complex IV assembly intermediates in WT mitochondria and mitochondria with a frameshift mutation in COX3 by BN PAGE and SILAC analysis. Their findings led to a preliminary assembly model for cytochrome $c$ oxidase and they characterized the proteins MR1-S, PET117 and PET100 as late stage assembly factors. PET100 was found to mediate the interaction between PET117 and MR1-S and with complex IV subunits. Furthermore, Aich et al. (Aich et al., 2018) characterized COX16 and found it to mediate the interaction of the copper chaperone SCO1 with COX2. Interestingly, COX16 also promotes assembly of COX2 with the COX1-containing late stage MITRAC complex, where MITRAC12 is still present, but C12ORF62 has already dissociated. Figure 16 shows a putative complex IV assembly model. 


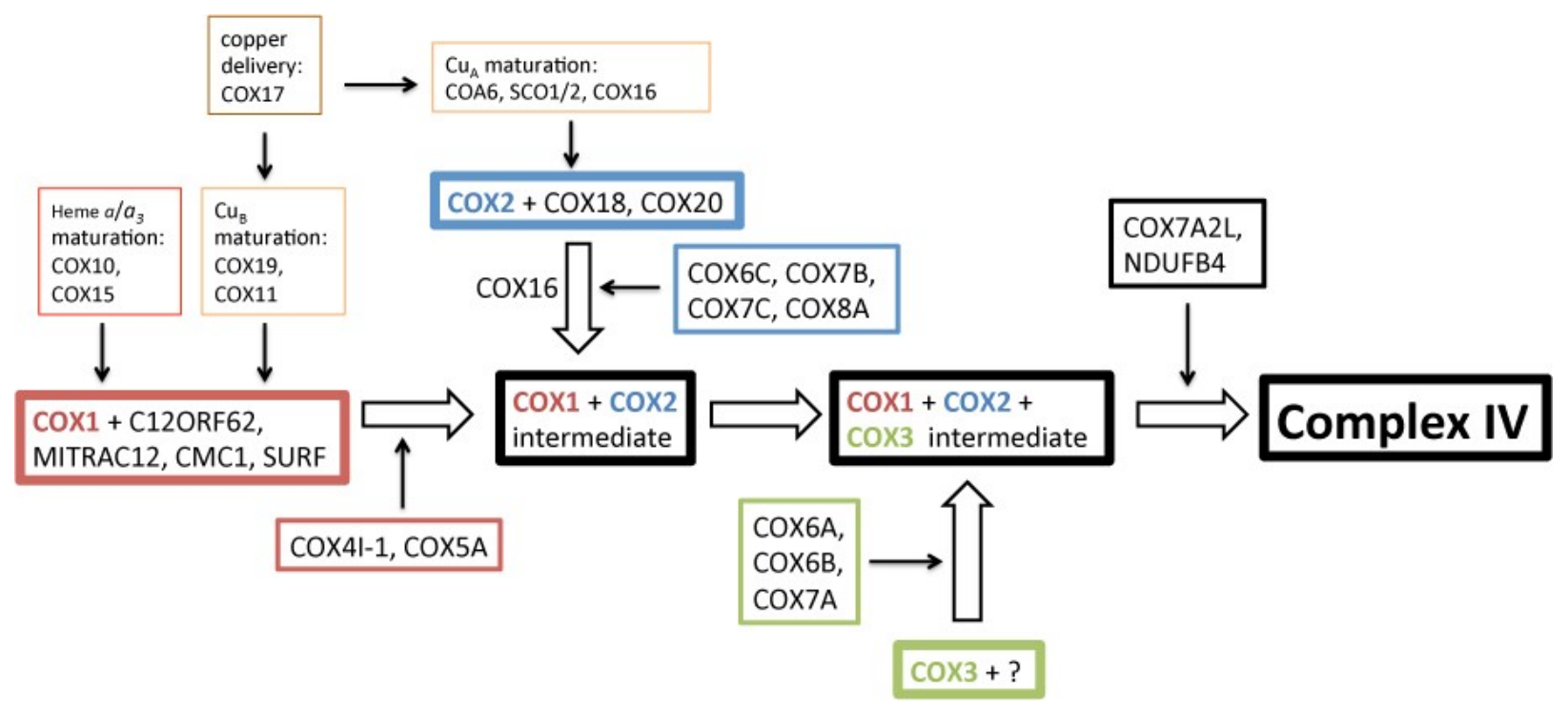

Figure 16 Putative model for the cytochrome $c$ oxidase assembly. Complex IV assembly works with the COX1 module as a core. After COX1 translation assisted by MITRAC12 and C12ORF62, COX1 transits into the early MITRAC complex with CMC1, MITRAC12, C12ORF62, SURF1, where the metal centres in COX1 mature with the assistance of COX10 and COX15 for heme $A$ and COX11 and COX19 for copper. The COX4I-1, COX5A and HIGD1A complex then provided the subunits COX4I-1 and COX5A, which assembles into MITRAC after the disassociation of CMC1. After the maturation of COX2 assisted by COX18, COX20, COA6, SCO1, SCO2 and COX16, COX16 promotes the assembly of the COX2 module with COX6C, COX7B, COX7C and COX8A onto late stage MITRAC. This is followed by the assembly of the COX3 module to the complex with COX6A, COX6B and COX7A2. The last step of complex IV assembly is the addition of NDUFA4 and COX7A2L. Modified after (Timón-Gómez et al., 2018). 


\subsection{Aims of this study}

The mitochondrial ribosome is unique machinery, which differs both from its bacterial ancestor and the cellular ribosome. Coevolved together with the mitochondrial and cellular genome, the mitochondrial ribosome is specialized in the synthesis of a small number of highly hydrophobic proteins still encoded by the mitochondrial genome. The translation in mitochondria and in the cytosol have to be coordinated to optimize the assembly of the OXPHOS complexes, which mostly consist of subunits of both mitochondrial and cytosolic translational origin. In yeast, specific cytosolic translated translations regulators are often needed by the mitochondrial ribosome to translate the mitochondrial-encoded proteins. Some of the translation regulators also participate in the assembly of OXPHOS complexes and therefore regulates mitochondrial translation via a feedback loop mechanism.

In human, little is known of translation regulators. One of the few characterized mitochondrial ribosome associated translations regulator is C12ORF62. This protein is essential for the translation of COX1 in human mitochondria and is also present in the MITRAC complex, which is an early assembly intermediate of the cytochrome $c$ oxidase. In this thesis, a C12ORF62 $2^{\text {FLAG }}$ cell line should be used to purify an early form of the MITRAC complex, while it is still associated with the mitochondrial ribosome. To accomplish this, the crude mitochondrial ribosome fraction has to be isolated and the MITRAC associated mitochondrial ribosome should be obtained by FLAG immunopurification.

MITRAC15 is a protein, which was detected in the MITRAC complex. It was shown to act in both complex IV and complex I assembly and is also present in an early assembly intermediate of the $P_{\mathrm{P}}-\mathrm{b}$ module. In this thesis, the function of MITRAC15 should be characterized further. Therefore, a MITRAC15 ${ }^{\text {FLAG }}$ cell line was created to investigate the interaction network of MITRAC15 and aMITRAC15 ${ }^{-/}$cell line was created to assess the functions of MITRAC15. 


\section{Materials and methods}

\subsection{Materials}

\subsubsection{Chemicals and enzymes}

Table 1 List of chemicals used in this study.

\begin{tabular}{|l|l|}
\hline Chemical & Supplier \\
\hline${ }^{35}$ S]-L-methionine & Hartmann Analytic \\
\hline 2-mercaptoethanol (B-mercaptoethanol) & Sigma-Aldrich \\
\hline 6-aminocaproic acid & Sigma-Aldrich \\
\hline Acetic acid & Roth \\
\hline Acetone & AppliChem or Merk \\
\hline Acrylamide/bisacrylamide (37.5:1) solution & Roth \\
(Rotiphorese ${ }^{8}$ Gel 30) & \\
\hline Acrylamide, $4 \times$ crystallized & Roth \\
\hline Adenine hemisulfate salt & Sigma-Aldrich \\
\hline ADP (adenosine-5'-diphosphate) & Sigma-Aldrich \\
\hline Agarose NEEO ultra-quality & Roth \\
\hline Ammonium acetate (NH${ }_{4}$ Ac) & Merck \\
\hline Ampicillin & AppliChem \\
\hline Anisomycin & Sigma-Aldrich \\
\hline Anti-FLAG M2 agarose beads & Sigma-Aldrich \\
\hline Antimycin A & Sigma-Aldrich \\
\hline ATP (adenosine-5'-triphosphate) & Roche \\
\hline Bacto ${ }^{\text {TM }}$ Agar & BD \\
\hline Bacto ${ }^{\text {TM }}$ Peptone & BD \\
\hline Bacto ${ }^{\text {TM }}$ Tryptone & BD \\
\hline Bacto Yeast Extract & BD \\
\hline N,N'-Methylene bisacrylamide & Roth \\
\hline Bis-Tris & AppliChem \\
\hline
\end{tabular}




\begin{tabular}{|l|l|}
\hline Bovine serum albumin (BSA) fatty acid free & Sigma-Aldrich \\
\hline Bromophenol Blue & Merck \\
\hline Calcium chloride(CaCl ) dihydrate & Roth \\
\hline Chloramphenicol & Roth \\
\hline cOmplete, EDTA-free protease inhibitor tablet & Roche \\
\hline Coomassie Brilliant Blue G-250 & Serva \\
\hline Coomassie Brilliant Blue R-250 & Serva \\
\hline Creatine kinase & Roche \\
\hline Creatine phosphate & Roche \\
\hline Diaminobenzidine (DAB) & Sigma-Aldrich \\
\hline DDM (n-Dodecyl-b-D-maltoside) & Sigma-Aldrich \\
\hline DMNG (Decyl Maltose Neopentyl Glycol) & Antrace \\
\hline Digitonin & Calbiochem \\
\hline DMEM (Dulbecco's Modified Eagle Medium) & Gibco \\
\hline DMP (dimethyl pimelimidate) & Sigma-Aldrich \\
\hline DMSO (dimethylsulfoxide) & AppliChem \\
\hline DNase I & Roche \\
\hline DTT (1,4-dithiothreitol) & Roth \\
\hline ECL Western Blotting detection reagent & GE-Healthcare \\
\hline EDTA (ethylene diamine tetraacetic acid) & Roth \\
\hline Emetine & Sigma \\
\hline Ethanol & Roth \\
\hline Ethidium bromide 0.07\% & AppliChem \\
\hline FastDigest Green Buffer (10X) & Thermo Scientific \\
\hline FLAG Peptide & Sigma-Aldrich \\
\hline Galactose, D(+) & Roth \\
\hline GeneJuice & Novogen \\
\hline GeneRuler DNA Ladder Mix & Fermentas \\
\hline Glucose, D(+) & \\
\hline L-Glutamine 100x & Roth \\
\hline
\end{tabular}




\begin{tabular}{|l|l|}
\hline Glycerol & Sigma-Aldrich \\
\hline Glycine & Roth \\
\hline GDN (Glyco-Diosgenin) & Antrace \\
\hline $\begin{array}{l}\text { HEPES } \\
\text { ethane-sulfonic acid) }\end{array}$ & Roth \\
\hline Herring sperm DNA & Promega \\
\hline KOD Hot Start DNA Polymerase-1-piperazine- & Novagen \\
\hline HMW calibration Kit & GE-Healthcare \\
\hline Hydrochloric acid (HCl) $37 \%$ & Roth \\
\hline Hydrogen peroxide solution & Sigma-Aldrich \\
\hline Hygromycin B & Invitrogen \\
\hline IgG from bovineserum & Bio-Rad \\
\hline IgG from human serum & Sigma-Aldrich \\
\hline Imidazole & Merck \\
\hline IPTG(Isopropyl $\beta$-D-1-thiogalactopyranoside) & Roth \\
\hline LMNG (Lauryl Maltose Neopentyl Glycol) & Antrace \\
\hline Lithium acetate (LiAc) & AppliChem \\
\hline Lipofectamine RNAiMAX & Invitrogen \\
\hline Luminol & Sigmal-Aldrich \\
\hline Lysozyme from chicken egg white & Sigma-Aldrich \\
\hline Magnesium chloride (MgCl ${ }_{2}$ ) heptahydrate & Merck \\
\hline Magnesium sulfate (MgSO ${ }_{4}$ ) heptahydrate & Roth \\
\hline Manganese (II)chloride $\left(\right.$ MnCl $_{2}$ ) tetrahydrate & Roth \\
\hline Methanol & Roth \\
\hline Milk powder & Grano Vita \\
\hline MOPS (morpholinopropanesulfonic acid) & Sigma-Aldrich \\
\hline NADH (nicotinamide adenine dinucleotide) & Roche \\
\hline Ni-NTA agarose & Qiagen \\
\hline NP-40 Substitute ( 4-Nonylphenyl-polyethylene & Sigma-Aldrich \\
\hline
\end{tabular}




\begin{tabular}{|c|c|}
\hline OBG (Octyl $\beta$-D-glucopyranoside) & Sigma-Aldrich \\
\hline Oligomycine & Sigma-Aldrich \\
\hline Oligonucleotides & Metabion \\
\hline Opti-MEM & Gibco \\
\hline Oxaloacetic acid & Sigma-Aldrich \\
\hline Penicillin-Streptomycin $(10,000 \mathrm{U} / \mathrm{mL})$ & Gibco \\
\hline PMSF (phenylmethanesulfonyluoride) & Roth \\
\hline Potassium acetate (KAc) & Merck \\
\hline Potassium chloride $(\mathrm{KCl})$ & Roth \\
\hline Potassium cyanide $(\mathrm{KCN})$ & Sigma-Aldrich \\
\hline Potassium dihydrogen phosphate $\left(\mathrm{KH}_{2} \mathrm{PO}_{4}\right)$ & Merck \\
\hline Di-potassium hydrogen phosphate $\left(\mathrm{K}_{2} \mathrm{HPO}_{4}\right)$ & Roth \\
\hline $\begin{array}{l}\text { PCC (4-trans-(4-trans-Propylcyclohexyl)- } \\
\text { cyclohexyl } \alpha \text {-maltoside) }\end{array}$ & Glycon \\
\hline Potassium hydroxid $(\mathrm{KOH})$ & Roth \\
\hline cOmplete $^{\mathrm{TM}}$ Protease Inhibitor Cocktail Tablets & Roche \\
\hline $\begin{array}{l}\text { Plus Protein }{ }^{\mathrm{TM}} \text { All Blue Prestained Protein } \\
\text { Standard was }\end{array}$ & Bio-Rad \\
\hline Proteinase K & Roche \\
\hline Protein-A Sepharose & GE-Healthcare \\
\hline Puromycin & Invivogen \\
\hline Roti-Quant ${ }^{\circledR}$ reagent & Roth \\
\hline Rotiphorese $®$ Gel $30(37,5: 1)$ & Roth \\
\hline Rubidium chloride $(\mathrm{RbCl})$ & Roth \\
\hline Saccharose & Roth \\
\hline SDS (sodium dodecyl sulfate) & Serva \\
\hline SDS marker broad range & Biorad \\
\hline Sodium azide & Sigma-Aldrich \\
\hline Sodium chloride $(\mathrm{NaCl})$ & Roth \\
\hline Sodium hydroxide $(\mathrm{NaOH})$ & Roth \\
\hline
\end{tabular}




\begin{tabular}{|l|l|}
\hline Sodium bicarbonate $\left(\mathrm{Na}_{2} \mathrm{CO}_{3}\right)$ & Sigma-Aldrich \\
\hline Sodium hydrogen carbonate $\left(\mathrm{NaHCO}_{3}\right)$ & Merck \\
\hline di-Sodium hydrogen phosphate $\left(\mathrm{Na}_{2} \mathrm{HPO}_{4}\right)$ & AppliChem \\
\hline Sodium pyruvate 100x & Sigma-Aldrich \\
\hline Sodium tetraborate & Merck \\
\hline Sorbitol & Roth \\
\hline D(+)-Sucrose, RNase Free and DNase Free & Roth \\
\hline Sulfuric acid $\left(\mathrm{H}_{2} \mathrm{SO}_{4}\right)$ & Merck \\
\hline TCA (trichloroacetic acid) & Merck \\
\hline Tetracycline & Roth \\
\hline TEMED (tetramethylethylenediamine) & Roth \\
\hline Trehalose & Roth \\
\hline Tricine & Roth \\
\hline Tris (tris(hydroxymethyl)aminomethane) & Roth \\
\hline Triton X-100 & Sigma-Aldrich \\
\hline TRlzol & Thermo Fisher \\
\hline Trypsin EDTA & Sigma-Aldrich \\
\hline Tween-20 & Roth \\
\hline Uracil & Sigma-Aldrich \\
\hline Uridine & Sigma-Aldrich \\
\hline USB® Taq DNA Polymerase & Affymetrix \\
\hline Unstained SDS PAGE Protein Marker $(6.5$ & Serva \\
\hline 200 kDa) & \\
\hline Urea & Roth \\
\hline Valinomycine & Sigma-Aldrich \\
\hline & \\
\hline
\end{tabular}




\subsubsection{Kits and disposals}

Table 2 List of Kits and disposals used in this study.

\begin{tabular}{|c|c|}
\hline Product & Supplier \\
\hline $\begin{array}{l}\text { Amicon }{ }^{\circledR} \text { Ultra-4 centrifugal filter unit } \\
10 \mathrm{~K} \text { MWCO }\end{array}$ & Millipore \\
\hline Blotting paper & Heinemann Labortechnik \\
\hline Cell culture flasks $25 \mathrm{~cm}^{2}, 75 \mathrm{~cm}^{2}$ & Thermo Fisher \\
\hline Cell culture plates $6,12,24,96$ well & Greiner \\
\hline CELLSTAR $^{\circledR}$ single cell cultre plates & Greiner \\
\hline CELLSTAR $^{\circledR}$ Centrifuge Tubes & Greiner Bio-One \\
\hline $\begin{array}{l}\text { Complex I Enzyme Activity Microplate } \\
\text { Assay Kit }\end{array}$ & abcam \\
\hline $\begin{array}{l}\text { Complex I Enzyme Activity Microplate } \\
\text { Assay Kit }\end{array}$ & abcam \\
\hline Fast Digest restriction enzymes & Fermentas \\
\hline $\begin{array}{llll}\left.\text { Flexi }^{(}\right) & \text {Rabbit } & \text { Reticulocyte } & \text { Lysate } \\
\text { System } & & & \\
\end{array}$ & Promega \\
\hline GeneRuler DNA Ladder Mix & Fermentas \\
\hline $\begin{array}{l}\text { High Pure PCR Template Preparation } \\
\text { Kit }\end{array}$ & Roche \\
\hline $\begin{array}{l}\text { Immobilon-P } \quad \text { Transfer } \\
\text { (PVDF) }\end{array}$ & Millipore \\
\hline KOD Hot Start DNA Polymerase & Merck \\
\hline MEDIX X-ray films & FOMA BOHEMIA \\
\hline Microtube $1.5 \mathrm{ml}$ and $2.0 \mathrm{ml}$ & Sarstedt \\
\hline Minisart syringe filters & Sartorius AG \\
\hline
\end{tabular}




\begin{tabular}{|l|l|}
\hline Pipette tips $10 \mu \mathrm{l}, 200 \mu \mathrm{l}$, and $1 \mathrm{ml}$ & Sarstedt \\
\hline QuickExtract ${ }^{\mathrm{TM}}$ DNA Extraction Solution & Lucigen \\
\hline Rapid DNA Ligation Kit & Thermo Scientific \\
\hline Resourse S column 5 ml Quick & GE Healthcare \\
\hline $\begin{array}{l}\text { Spin columns Mobicol "classic" } \\
\text { Transcription/Translation System }{ }^{\circledR}\end{array}$ & MoBiTec \\
\hline $\begin{array}{l}\text { Wizard }{ }^{\circledR} \text { Plus SV Gel and PCR DNA } \\
\text { Purification System }\end{array}$ & Promega \\
\hline $\begin{array}{l}\text { Wizard }{ }^{\circledR \quad P l u s ~ S V ~ M i n i p r e p s ~ D N A ~} \\
\text { Purification System }\end{array}$ & Promega \\
\hline
\end{tabular}

\subsubsection{Buffers and solutions}

Table 3 List of buffers and solutions used in this study.

\begin{tabular}{|l|l|}
\hline Buffer/Solution & Composition \\
\hline Acetate buffer & $0.5 \mathrm{M} \mathrm{NH}_{4}$ Ac/acetic acid $\mathrm{pH} 3.5$ \\
\hline Agarose gel solution & $1 \%$ agarose, TAE buffer \\
\hline AVO mix & $\begin{array}{l}1 \mathrm{mM} \text { antimycin, } 0.1 \mathrm{mM} \text { valinomycin } \\
2 \mathrm{mM} \text { oligomycin }\end{array}$ \\
\hline Blocking solution & $5 \%-10 \%$ milk powder in TBST \\
\hline Blotting buffer & $\begin{array}{l}25 \mathrm{mM} \text { Tris, } 192 \mathrm{mM} \text { glycine, } 10 \% \\
\mathrm{methanol}\end{array}$ \\
\hline BN acrylamide & $48 \%$ acrylamide, $1.5 \%$ bis-acrylamide \\
\hline BN anode buffer & $50 \mathrm{mM}$ Bis-Tris/HCl pH 7.0 \\
\hline BN clear cathode buffer & $50 \mathrm{mM}$ tricine, $15 \mathrm{mM}$ Bis-Tris \\
\hline
\end{tabular}




\begin{tabular}{|c|c|}
\hline BN blue cathode buffer & $\begin{array}{l}50 \mathrm{mM} \text { tricine, } 15 \mathrm{mM} \text { Bis-Tris, 0.2\% } \\
\text { Coomassie Brilliant Blue G-250 }\end{array}$ \\
\hline BN gel buffer (3X) & $\begin{array}{l}200 \mathrm{mM} \text { 6-aminocaproic acid, } 150 \mathrm{mM} \\
\text { Bis-Tris/HCl pH } 7.0\end{array}$ \\
\hline BN loading buffer (10X) & $\begin{array}{l}\text { 5\% Coomassie Brilliant Blue G-250, } \\
500 \mathrm{mM} \\
6 \text {-aminocaproic acid, } 100 \text { mM Bis- } \\
\text { Tris/HCl pH } 7.0\end{array}$ \\
\hline $\begin{array}{l}\text { BN solubilization buffer } \\
\text { (EDTA) }\end{array}$ & $\begin{array}{l}20 \mathrm{mM} \text { Tris/HCl, } 60 \mathrm{mM} \mathrm{NaCl}, 10 \% \\
\text { glycerol, } 1 \mathrm{mM} \text { EDTA, } 1 \mathrm{mM} \text { PMSF with } \\
1 \% \text { digitonin or } 0.6 \% \text { DDM }\end{array}$ \\
\hline $\begin{array}{l}\mathrm{BN} \text { solubilization buffer } \\
\left(\mathrm{MgCl}_{2}\right)\end{array}$ & $\begin{array}{l}20 \mathrm{mM} \text { Tris/HCl, } 60 \mathrm{mM} \mathrm{NaCl}, 10 \% \\
\text { glycerol, } 20 \mathrm{mM} \mathrm{MgCl} \text {, } 1 \mathrm{mM} \mathrm{PMSF} \\
\text { with } 1 \% \text { digitonin or } 0.6 \% \text { DDM }\end{array}$ \\
\hline Cell culture medium & $\begin{array}{l}\text { DMEM (Dulbecco's Modified Eagle } \\
\text { Medium) supplemented with } 10 \%(\mathrm{v} / \mathrm{v}) \\
\text { fetal bovine serum, } 1 \mathrm{mM} \text { sodium } \\
\text { pyruvate, } 2 \mathrm{mM} \text { L-Glutamine, } 50 \mu \mathrm{g} / \mathrm{ml} \\
\text { uridine and with or without penicillin } \\
\text { streptomycin (filtered) }\end{array}$ \\
\hline $\begin{array}{l}\text { Coomassie destaining } \\
\text { solution }\end{array}$ & $40 \%$ ethanol, $10 \%$ acetic acid \\
\hline Coomassie staining solution & $\begin{array}{l}0.25 \% \text { Coomassie Brilliant Blue R-250, } \\
40 \% \text { ethanol, } 10 \% \text { acetic acid }\end{array}$ \\
\hline Digitonin stock solution & $5 \%$ digitonin in $\mathrm{H}_{2} \mathrm{O}$ \\
\hline DNA loading dye & $10 \%$ saccharose, $0.25 \%$ OrangeG \\
\hline DTT buffer & $\begin{array}{l}10 \mathrm{mM} \text { DTT, } 100 \mathrm{mM} \text { Tris } / \mathrm{H}_{2} \mathrm{SO}_{4} \mathrm{pH} \\
9.4\end{array}$ \\
\hline Fast lysate buffer & $\begin{array}{l}150 \mathrm{mM} \mathrm{NaCl}, 20 \mathrm{mM} \mathrm{MgCl}_{2}, 1 \mathrm{mM} \\
\mathrm{PMSF}, 50 \mathrm{mM} \text { Tris/HCl pH } 7.4 \text { with } 1 \% \\
\text { NP-40 Substitute, cOmplete } \\
\text { Protease Inhibitor Cocktail Tablets } \\
1 / 50 \mathrm{ml}\end{array}$ \\
\hline Gel filtration buffer & $\begin{array}{l}150 \mathrm{mM} \mathrm{NaCl}, 10 \% \text { glycerol, } 20 \mathrm{mM} \\
\mathrm{MgCl}_{2}, 1 \mathrm{mM} \text { PMSF, } 50 \mathrm{mM} \text { Tris/HCl } \\
\mathrm{pH} 7.4 \text { with } 0.05 \% \text { PCC }\end{array}$ \\
\hline Homogenization buffer & $\begin{array}{l}0.6 \mathrm{M} \text { sorbitol, } 1 \mathrm{mM} \text { EDTA, } 0.2 \% \text { fatty } \\
\text { acid free BSA, } 1 \mathrm{mM} \text { PMSF, } 10 \mathrm{mM} \\
\text { Tris/HCl pH } 7.4\end{array}$ \\
\hline Hypotonic buffer (import) & $\begin{array}{l}100 \mathrm{mM} \text { sucrose, } 1 \mathrm{mM} \text { EGTA, } 10 \mathrm{mM} \\
\text { MOPS, pH } 7.2\end{array}$ \\
\hline
\end{tabular}




\begin{tabular}{|c|c|}
\hline Hypertonic buffer (import) & $1.25 \mathrm{M}$ sucrose, $10 \mathrm{mM}$ MOPS, pH 7.2 \\
\hline Import buffer & $\begin{array}{l}250 \mathrm{mM} \text { sucrose, } 80 \mathrm{mM} \mathrm{KAcO}, 5 \mathrm{mM} \\
\mathrm{Mg}(\mathrm{AcO})_{2}, 5 \mathrm{mM} \text { methionine, } 10 \mathrm{mM} \\
\text { sodium succinate, } 20 \mathrm{mM} \text { HEPES, pH } \\
7.4\end{array}$ \\
\hline Isolation buffer (import) & $\begin{array}{l}225 \text { mM sucrose, } 75 \text { mM mannitol, } \\
1 \text { mM EGTA, } 10 \text { mM MOPS, pH } 7.2\end{array}$ \\
\hline PMSF stock & $0.2 \mathrm{M}$ PMSF in ethanol \\
\hline $\begin{array}{l}\text { Potassium phosphate (KPi) } \\
\text { buffer }\end{array}$ & $80.2 \% \mathrm{~K}_{2} \mathrm{HPO}_{4}, 19.8 \% \mathrm{KH}_{2} \mathrm{PO}_{4}$ \\
\hline Resolving gel (SDS PAGE) & $\begin{array}{l}\text { 10-16\% acrylamide (Rotiphorese }{ }^{\circledR} \text { Gel } \\
\text { 30), } 0.05 \% \text { TEMED, } 0.1 \% \text { APS, } 0.1 \% \\
\text { SDS, } 386 \mathrm{mM} \text { Tris/HCl pH } 8.8\end{array}$ \\
\hline Resolving gel (Urea PAGE) & $\begin{array}{l}17.5 \% \text { acrylamide, } 0.23 \% \text { bis- } \\
\text { acrylamide, } \\
5.4 \mathrm{M} \text { urea, } 8 \mathrm{mM} \mathrm{NaCl}, 0.09 \% \text { SDS, } \\
0.1 \% \mathrm{APS}, 0.05 \% \text { TEMED, } \\
684 \mathrm{mM} \text { Tris/HCl pH } 8.8\end{array}$ \\
\hline RF1 buffer & $\begin{array}{l}100 \mathrm{mM} \mathrm{RbCl}, 50 \mathrm{mM} \mathrm{MnCl}_{2}, 30 \mathrm{mM} \\
\text { sodium acetate, } 15 \mathrm{mM} \mathrm{CaCl}_{2}, 15 \% \\
\text { glycerol, pH } 5.8\end{array}$ \\
\hline RF2 buffer & $\begin{array}{l}10 \mathrm{mM} \mathrm{RbCl}, 50 \mathrm{mM} \mathrm{MnCl}_{2}, 10 \mathrm{mM} \\
\mathrm{MOPS}, 75 \mathrm{mM} \mathrm{CaCl}_{2}, 15 \% \text { glycerol, } \\
\text { pH } 6.8\end{array}$ \\
\hline SDS loading buffer & $\begin{array}{l}10 \% \text { glycerol, } 2 \% \text { SDS, } 0.01 \% \\
\text { bromophenole blue, } 1 \% \beta \text { - } \\
\text { mercaptoethanol, } \\
60 \mathrm{mM} \text { Tris } / \mathrm{HCl} \mathrm{pH} 6.8\end{array}$ \\
\hline SDS running buffer & $\begin{array}{l}25 \mathrm{mM} \text { Tris, } 192 \mathrm{mM} \text { glycine, } 0.1 \% \\
\text { SDS }\end{array}$ \\
\hline SEM buffer & $\begin{array}{l}250 \mathrm{mM} \text { saccharose, } 1 \text { mM EDTA, } \\
10 \mathrm{mM} \text { MOPS/KOH pH } 7.2\end{array}$ \\
\hline Stacking gel (SDS PAGE) & $\begin{array}{l}4 \% \text { arylamide, } 0.1 \% \text { SDS, } 0.1 \% \text { APS, } \\
0.05 \% \text { TEMED, } 80 \mathrm{mM} \text { Tris/HCl pH } 6.8\end{array}$ \\
\hline Stacking gel (Urea PAGE) & $\begin{array}{l}5.4 \% \text { arylamide, } 0.07 \% \text { bis-acrylamide, } \\
0.12 \% \text { SDS, } 3.33 \mathrm{M} \text { urea, } 0.1 \% \mathrm{APS}, \\
0.05 \% \text { TEMED, } 109 \mathrm{mM} \text { Tris/HCl pH } \\
6.8\end{array}$ \\
\hline $\begin{array}{l}\text { Solubilization buffer } \\
\text { (sucrose cushion) }\end{array}$ & $\begin{array}{l}150 \mathrm{mM} \mathrm{NaCl}^{2} 260 \mathrm{mM} \text { sucrose, } \\
20 \mathrm{mM} \mathrm{MgCl}, 1 \mathrm{mM} \text { PMSF, } 50 \mathrm{mM} \\
\text { Tris/HCl pH } 7.4 \text { with } 0.5 \% \text { PCC }\end{array}$ \\
\hline
\end{tabular}




\begin{tabular}{|c|c|}
\hline $\begin{array}{l}\text { Solubilization buffer } \\
\text { (immunoprecipitation) }\end{array}$ & $\begin{array}{l}150 \mathrm{mM} \mathrm{NaCl}, 10 \% \text { glycerol, } 20 \mathrm{mM} \\
\mathrm{MgCl}_{2}, 1 \mathrm{mM} \text { PMSF, } 50 \mathrm{mM} \text { Tris/HCl } \\
\mathrm{pH} 7.4 \text { with } 1 \% \text { digitonin or } 0.5 \% \mathrm{PCC}\end{array}$ \\
\hline Sucrose cushion & $\begin{array}{l}\text { Sucrose } 34 \%, 150 \mathrm{mM} \mathrm{NaCl}, 20 \mathrm{mM} \\
\mathrm{MgCl}_{2}, 50 \mathrm{mM} \text { Tris/ } \mathrm{HCl} \mathrm{pH} \mathrm{7.4,} 0.1 \% \\
\text { PCC, cOmplete } \\
\text { Cocktail Tablets } 1 / 50 \mathrm{ml}\end{array}$ \\
\hline TAE buffer & $\begin{array}{l}2 \mathrm{mM} \text { EDTA, } 40 \mathrm{mM} \text { Tris/acetic acid } \mathrm{pH} \\
8.0\end{array}$ \\
\hline TBS (Tris-Buffered Saline) & $150 \mathrm{mM} \mathrm{NaCl}, 50 \mathrm{mM}$ Tris/HCl pH 7.5 \\
\hline TBST (TBS and Tween-20) & $\begin{array}{l}150 \mathrm{mM} \mathrm{NaCl}, 0.05 \% \text { Tween-20, } \\
50 \mathrm{mM} \mathrm{Tris/HCl} \mathrm{pH} \mathrm{7.5}\end{array}$ \\
\hline TCA solution & $100 \%$ TCA in water \\
\hline TE buffer & $1 \mathrm{mM}$ EDTA, $10 \mathrm{mM}$ Tris/HCl pH 8.0 \\
\hline TfB1 buffer & $\begin{array}{l}30 \mathrm{mM} \mathrm{KAc}, 100 \mathrm{mM} \mathrm{RbCl}, 10 \mathrm{mM} \\
\mathrm{CaCl}_{2} \text {, } \\
50 \mathrm{mM} \mathrm{MnCl}, 15 \% \text { glycerol/acetic acid } \\
\mathrm{pH} 5.8\end{array}$ \\
\hline TfB2 buffer & $\begin{array}{l}10 \mathrm{mM} \mathrm{RbCl}, 75 \mathrm{mM} \mathrm{CaCl}_{2}, 15 \% \\
\text { glycerol, } \\
10 \mathrm{mM} \text { MOPS/KOH pH } 6.5\end{array}$ \\
\hline TH buffer & $\begin{array}{l}300 \mathrm{mM} \text { Trehalose } 10 \mathrm{mM} \mathrm{KCl}, 10 \mathrm{mM} \\
\text { HEPES, pH } 7.4\end{array}$ \\
\hline Urea PAGE acrylamide & $\begin{array}{l}60 \% \text { acrylamide, } 0.8 \% \text { bis-acrylamide } \\
\text { in } \mathrm{H}_{2} \mathrm{O}\end{array}$ \\
\hline Urea PAGE running buffer & $\begin{array}{l}50 \mathrm{mM} \text { Tris, } 192 \mathrm{mM} \text { glycine, } 0.1 \% \\
\text { SDS }\end{array}$ \\
\hline $\begin{array}{l}\text { Washing buffer } \\
\text { (Immunoprecipitation) }\end{array}$ & $\begin{array}{l}150 \mathrm{mM} \mathrm{NaCl}, 10 \% \text { glycerol, } 20 \mathrm{mM} \\
\mathrm{MgCl}_{2}, 1 \mathrm{mM} \mathrm{PMSF}, 50 \mathrm{mM} \text { Tris/HCl } \\
\mathrm{pH} 7.4 \text { with } 0.1 \% \text { digitonin or } 0.05 \% \\
\text { PCC }\end{array}$ \\
\hline Washing buffer (cryoEM) & $\begin{array}{l}150 \mathrm{mM} \mathrm{NaCl}, 0.5 \% \text { glycerol, } 20 \mathrm{mM} \\
\mathrm{MgCl}_{2}, 1 \mathrm{mM} \text { PMSF, } 50 \mathrm{mM} \text { HEPES } \\
\mathrm{pH} 7.4 \text { with } 0.1 \% \text { digitonin or } 0.05 \% \\
\text { PCC }\end{array}$ \\
\hline
\end{tabular}




\subsubsection{Antibodies}

Primary polyclonal antibodies were cultivated in rabbit by the injection of synthetic peptides or purified proteins. Goat anti-rabbit coupled to horseradish-peroxidase (HRP) (Dianova) was used as secondary antibody for detection.

\subsubsection{Plasmids and Oligonucleotides}

Plasmids used in this work were enriched in competent Escherichia coli (E. coli) XL1-Blue cells (Stratagene).

Table 4 List of plasmids used in this study.

\begin{tabular}{|l|l|l|l|}
\hline Plasmid & Backbone & Insert & Source \\
\hline pX330 & - & - & $\begin{array}{l}\text { (Ann Ran et al., } \\
\text { 2013) }\end{array}$ \\
\hline pOTB7-COX6A1 & pOTB7 & COX6A1 & (Mick et al., 2012) \\
\hline pcDNA5-NDUFB8 & pcDNA5 & NDUFB8 & This study \\
\hline pEGFP-N1 & - & - & Clonetech \\
\hline
\end{tabular}

Primer and siRNA used in this study are listed in Table 5. DNA primers were synthesized by Microsynth Seqlab and siRNA were provided by Eurogentec.

Table 5 Oligonnucleoties used in this study.

\begin{tabular}{|l|l|l|l|}
\hline Oligonucleotide & Species & Sequence & Function \\
\hline CW-P1 & DNA & $\begin{array}{l}\text { GGATTTAGGTGACAC } \\
\text { TATAGAATACATGGC } \\
\text { GGTGGCCAGGGCC }\end{array}$ & $\begin{array}{l}\text { Cloning of NDUFB8 for } \\
\text { lysate generation } \\
\text { (forward primer) }\end{array}$ \\
\hline CW-P2 & DNA & $\begin{array}{l}\text { TATAGTCGACTCAG } \\
\text { ATCTCATAGTGAAC } \\
\text { CACCCGCTCTGG }\end{array}$ & $\begin{array}{l}\text { Cloning of NDUFB8 for } \\
\text { lysate generation } \\
\text { (reverse primer) }\end{array}$ \\
\hline
\end{tabular}




\begin{tabular}{|l|l|l|l|}
\hline siTIM21 & RNA & $\begin{array}{l}\text { a) CCCAGGUGGUGA } \\
\text { AUAU } \\
\text { b) AUAUCACCACUUC } \\
\text { CUGGG }\end{array}$ & $\begin{array}{l}\text { siRNA for TIM21 } \\
\text { knockdown }\end{array}$ \\
\hline siACAD9 & RNA & $\begin{array}{l}\text { a) GGUCGUUGAUUC } \\
\text { UGAUGGA } \\
\text { b) UCCAUCAGAAUC } \\
\text { AACGAC }\end{array}$ & $\begin{array}{l}\text { siRNA for ACAD9 } \\
\text { knockdown }\end{array}$ \\
\hline $\begin{array}{l}\text { Non targeted } \\
\text { siRNA control }\end{array}$ & RNA & - & $\begin{array}{l}\text { Non targeted siRNA } \\
\text { control }\end{array}$ \\
\hline
\end{tabular}

\subsubsection{Cell lines}

Table 6 Cell lines used in this study.

\begin{tabular}{|c|c|}
\hline Cellline & Source \\
\hline $\begin{array}{l}\text { HEK293-Flp-In }{ }^{\text {TM }} \text { T-REx }^{\text {TM }} \text { (HEK239T)- } \\
\text { MITRAC15-FLAG }\end{array}$ & (Mick et al., 2012) \\
\hline $\begin{array}{l}\text { HEK293-Flp-In }{ }^{\text {TM }} \text { T-REx }^{\text {TM }} \text { (HEK239T)- } \\
\text { C12ORF62-FLAG }\end{array}$ & $\begin{array}{l}\text { (Richter-Dennerlein et al., } \\
\text { 2016). }\end{array}$ \\
\hline $\begin{array}{l}\text { HEK293-Flp-In }{ }^{\text {TM }} \text { T-REx }^{\text {TM }} \text { (HEK239T)- } \\
\text { MITRAC15 }^{-/-}\end{array}$ & This study \\
\hline
\end{tabular}

\subsubsection{Equipment and software}

Table 7 List of Instruments used in this study.

\begin{tabular}{|l|l|}
\hline Instrument & Manufacturer \\
\hline 5417 R (centrifuge) & Eppendorf \\
\hline 5424 (centrifuge) & Eppendorf \\
\hline 5804 R (centrifuge) & Eppendorf \\
\hline Sorvall RC 6 Plus (centrifuge) & Thermo Scientific \\
\hline JA-20 Rotor & Beckman Coulter \\
\hline ÄKTA purifier (FPLC) & GE Healthcare \\
\hline
\end{tabular}




\begin{tabular}{|l|l|}
\hline $\begin{array}{l}\text { HiLoad 16/600 Superdex 200 pg } \\
\text { (Column) }\end{array}$ & GE Healthcare \\
\hline Superdex 200 10/300 GL (Column) & GE Healthcare \\
\hline Curix 60 (developing machine) & AGFA \\
\hline EPS 601 power supply & GE Healthcare \\
\hline $\begin{array}{l}\text { GeneAmp PCR Systeme 2700 (thermo } \\
\text { cycler) }\end{array}$ & Applied Biosystems \\
\hline Heraeus ${ }^{\circledR}$ Hera cell 150 (incubator) & Thermo Scientific \\
\hline Heraeus $®$ Hera safe (sterile hood) & Thermo Scientific \\
\hline $\begin{array}{l}\text { Homogen-plus semiautomatic } \\
\text { homogeniser }\end{array}$ & Schuett biotech \\
\hline Hoefer SE600 Ruby Blue native system & GE Healthcare \\
\hline Light microscope & Zeiss \\
\hline NanoVueTM Spectrophotometer & GE Healthcare \\
\hline Optima L-90K Ultracentrifuge & Beckman Coulter \\
\hline SW 41 Ti Rotor & Beckman Coulter \\
\hline Potter S (Dounce homogenizer) & Sartorius \\
\hline Semi Dry Blotting Chamber & $\begin{array}{l}\text { PEQLAB } \\
\text { Biotechnologie }\end{array}$ \\
\hline $\begin{array}{l}\text { Amersham Typhoon Gel and Blot } \\
\text { Imaging Systems }\end{array}$ & GE Healthcare \\
\hline Storage Phosphor screen & GE Healthcare \\
\hline Thermomixer comfort & Eppendorf \\
\hline Typhoon FLA 9500 Phosphoimager & GE Healthcare \\
\hline Universal 320 (centrifuge) & Hettich \\
\hline VortexGenie2 & Scientific Industries \\
\hline
\end{tabular}


Table 8 Software used in this study.

\begin{tabular}{|l|l|}
\hline Software & Producer \\
\hline Adobe Illustrator CS5 & Adobe \\
\hline Adobe Photoshop CS5 & Adobe \\
\hline Data Graph 3.1.1. & Visual Data Tools \\
\hline Geneious R11 & Biomatters \\
\hline ImageQuant TL & GE Healthcare \\
\hline ChimeraX & UCSF \\
\hline Papers 3 & Mekentosj \\
\hline
\end{tabular}

\subsection{Methods}

\subsubsection{Cultivation of human cells}

HEK293-FIp-In ${ }^{\text {TM }}$ T-REx ${ }^{\text {TM }}$ (HEK239T) cell lines were cultured in DMEM supplemented as described in Table 3 . Cell lines were cultivated at $37^{\circ} \mathrm{C}$ under a $5 \% \mathrm{CO}^{2}$ humidified atmosphere and passaged upon reaching confluence. Cells were harvested using PBS with $1 \mathrm{mM}$ EDTA and reseeded or harvested using PBS and processed afterwards or stored at $-20^{\circ} \mathrm{C}$.

\subsubsection{Generation of knockout cell lines using the CRISPR-Cas9 system}

\subsubsection{Design of oligonucleotides}

The target within the gene of interest was selected using software provided by the lab of Feng Zhang(Hsu et al., 2013) (http://crispr.mit.edu/). And the top three results were used for the generation of knockouts. The selected guide sequenced were modified the following way for cloning into a pX330 vector ( $N=$ guide sequence, $\underline{N}=$ complement to guide sequence):

5'- CACC (G) NNNNNNNNNNNNNNNNNNNN -3'

3'- (C) NNNNNNNNNNNNNNNNNNNNCAAA -5' 


\subsubsection{Cloning of oligonucleotides into the $p X 330$ vector}

$5 \mu \mathrm{l}$ of each oligo (forward and reverse, $100 \mu \mathrm{M}$ ) were mixed with $30 \mu \mathrm{l}$ of water and $10 \mu \mathrm{l}$ of $5 \mathrm{x}$ ligation buffer from the Rapid DNA Ligation Kit (ThermoScientific). The mixture was heated $5 \mathrm{~min}$ at $95^{\circ} \mathrm{C}$ and the temperature was decreased $5{ }^{\circ} \mathrm{C} / \mathrm{min}$ until $20^{\circ} \mathrm{C} .1 \mu \mathrm{g}$ of the pX330 vector were digested with $1 \mu \mathrm{L} \mathrm{Bbsl}$ (ThermoScientific) for 30 minutes and purified with the Wizard ${ }^{\circledR}$ Plus SV Gel and PCR DNA Purification System. $2 \mu$ of the annealed oligonucleotide duplex was ligated with $50 \mathrm{ng}$ of the cutted pX330 vector and amplified in competent $E$. coliXL1-Blue cells (described in XXX).

\subsubsection{Transfection of HEK293T cells}

Cells were seeded in a 6 well plate $2 \mathrm{~d}$ before transfection. The confluence on the day of the transfection should be around $40 \%$ in the plate. $100 \mu \mathrm{l}$ OptiMEM, $5 \mu \mathrm{l}$ Gene Juice, $2 \mu \mathrm{g}$ of pX330 plasmids with guide and $400 \mathrm{ng}$ enhanced GFP plasmid (pEGFP-N1; Clonetech Laboratories, Inc) were mixed and incubated $20 \mathrm{~min}$ at room temperature. $600 \mu \mathrm{l}$ medium without penicillin and streptomycin was added to the mixture and it was transferred onto the cells after removal of media from the 6 well plate. After $3 \mathrm{~h}$ incubation at $37^{\circ} \mathrm{C}, 4 \mathrm{ml}$ medium without penicillin and streptomycin was added to the cells. After 3 days, cells were harvested with and sorted.

\subsubsection{Cell sorting and expansion}

Three days after transfection, cells were harvested with PBS containing $1 \mathrm{mM}$ EDTA. Cells were kept in PBS/1 mM EDTA at $37^{\circ} \mathrm{C}$ until sorting. Cells were sorted using a FACS Vantage SE by Sabrina Becker (UMG Cell sorting facility). GFP positive cells were sorted into 96 well plates and after the single colonies grew to a sufficient size, they were transferred to a 24 well plate. Cells were analyzed by Western blotting and clones, which does not display the protein of interest were further analyzed by sequencing. Clones which show a homozygous stop codon insertion in the target gene were chosen for further analysis. 


\subsubsection{Labelling of newly synthesized mitochondrial proteins using $\left[{ }^{35} \mathrm{~S}\right]$ methionine}

Protocolls for $\left[{ }^{35} \mathrm{~S}\right]$ methioninelabelling of newly synthesized mitochondrial proteins are modified after (Jeffreys and Craig, 1976).

\subsubsection{Pulse labelling}

Cells were seeded in $25 \mathrm{~cm}^{2}$ flasks $3 \mathrm{~d}$ before labelling. Media was removed from cells and $2 \mathrm{ml} \mathrm{FCS/methionine} \mathrm{free} \mathrm{media} \mathrm{were} \mathrm{added} \mathrm{to} \mathrm{the} \mathrm{cells.} \mathrm{After}$ 10 min incubation at $37^{\circ} \mathrm{C}$, the media was removed from the cells and $2 \mathrm{ml}$ FCS/Met free media were added to the cells again. After another round of 10 min incubationat $37^{\circ} \mathrm{C}$, the media was removed and $1,5 \mathrm{ml} \mathrm{FCS}$ containing methionine free media with $100 \mu \mathrm{g}$ emetin $/ 1 \mathrm{ml}$ media was added to the cells. After 10 min incubation at $37^{\circ} \mathrm{C}, 500$ media was taken out of the flasks and $20 \mu \mathrm{l}\left[{ }^{35} \mathrm{~S}\right]$ methionine (Hartmann Analytic) was added to the media. After $1 \mathrm{~h}$ incubation at $37^{\circ} \mathrm{C}$, the media was removed and cells were harvested and wash twice with PBS.

\subsubsection{Pulse chase labelling}

Cells were seeded in $25 \mathrm{~cm}^{2}$ flasks $3 \mathrm{~d}$ before labelling. Media was removed from cells and $2 \mathrm{ml} \mathrm{FCS/methionine} \mathrm{free} \mathrm{media} \mathrm{were} \mathrm{added} \mathrm{to} \mathrm{the} \mathrm{cells.} \mathrm{After}$ 10 min incubation at $37^{\circ} \mathrm{C}$, the media was removed from the cells and $2 \mathrm{ml}$ FCS/Met free media were added to the cells again. After another round of 10 min incubationat $37^{\circ} \mathrm{C}$, the media was removed and $1,5 \mathrm{ml} \mathrm{FCS} \mathrm{containing}$ methionine free media with $100 \mu \mathrm{g}$ anisomycin $/ 1 \mathrm{ml}$ media was added to the cells. After $10 \mathrm{~min}$ incubation at $37^{\circ} \mathrm{C}, 500$ media was taken out of the flasks and $20 \mu \mathrm{l}\left[{ }^{35} \mathrm{~S}\right]$ methionine (Hartmann Analytic) was added to the media. After $1 \mathrm{~h}$ incubation at $37^{\circ} \mathrm{C}$, the media was removed and $10 \mathrm{ml}$ media with penicillin and streptomycin were added to the cells. After the chasing time, the media was removed and cells were harvested and wash twice with PBS.

\subsubsection{Pulse labelling with puromycin treatment}

Cells were seeded in $25 \mathrm{~cm}^{2}$ flasks $3 \mathrm{~d}$ before labelling. Media was removed from cells and $2 \mathrm{ml} \mathrm{FCS/methionine} \mathrm{free} \mathrm{media} \mathrm{were} \mathrm{added} \mathrm{to} \mathrm{the} \mathrm{cells.} \mathrm{After}$ $10 \mathrm{~min}$ incubation at $37^{\circ} \mathrm{C}$, the media was removed from the cells and $2 \mathrm{ml}$ FCS/Met free media were added to the cells again. After another round of 10 
min incubationat $37^{\circ} \mathrm{C}$, the media was removed and $1,5 \mathrm{ml}$ FCS containing methionine free media with $100 \mu \mathrm{g}$ emetin $/ 1 \mathrm{ml}$ media was added to the cells. After 10 min incubation at $37^{\circ} \mathrm{C}, 500$ media was taken out of the flasks and $20 \mu \mathrm{l}\left[{ }^{35} \mathrm{~S}\right]$ methionine (Hartmann Analytic) were added to the media. After $10 \mathrm{~min}$ incubation at $37^{\circ} \mathrm{C}, 2 \mu \mathrm{g} / \mathrm{ml}$ puromycin was added to the media.After 20 min incubation at $37^{\circ} \mathrm{C}$, the media was removed and cells were harvested and wash twice with PBS.

\subsubsection{Isolation of human mitochondria}

Protocols of isolation of human mitochondria were modified after(Mick et al., 2012) and protocol for isolation of human mitochondria for import was modified afterprotocolls from Alexander Panov (Panov,2013).

\subsubsection{Isolation of human mitochondria}

Cells were harvested in PBS and pelleted $15 \mathrm{~min}$ at $4700 \mathrm{rpm}$ and $4{ }^{\circ} \mathrm{C}$. Cell pellets were resuspended in TH buffer with $0.1 \mathrm{mg} \mathrm{BSA} / \mathrm{ml}$ and pottered $30 \mathrm{x}$ at $800 \mathrm{rpm}$ on ice. Centrifugation steps were carried out in reaction tubes with a $5417 \mathrm{R}$ centrifuge (Eppendorf). Cellular debris was pelleted $10 \mathrm{~min}$ at $400 \mathrm{~g}$ and $4{ }^{\circ} \mathrm{C}$. The supernatant (SN1) containing mitochondria was collected. The cellular debris was resuspended in $\mathrm{TH}$ buffer with $0.1 \mathrm{mg} \mathrm{BSA} / \mathrm{ml}$ and pottered again $30 \mathrm{x}$ at $800 \mathrm{rpm}$ on ice and pelleted $10 \mathrm{~min}$ at $400 \mathrm{~g}$ and $4{ }^{\circ} \mathrm{C}$. The pellet was discarded and the supernatant (SN2) was added to SN1 and pelleted $7 \mathrm{~min}$ at $800 \mathrm{~g}$ and $4{ }^{\circ} \mathrm{C}$. The pellet was discarded and the new supernatant (SN3) was collected and pelleted $10 \mathrm{~min}$ at $11000 \mathrm{~g}$ and $4{ }^{\circ} \mathrm{C}$. The new pellet containing mitochondria was washed with $\mathrm{TH}$ buffer without BSA by resuspeinding it and pelleted $5 \mathrm{~min}$ at $11000 \mathrm{~g}$ and $4{ }^{\circ} \mathrm{C}$. The washed mitochondria were resuspended in $\mathrm{TH}$ buffer without BSA and quantified. They were used immediately or stored at $-80^{\circ} \mathrm{C}$.

\subsubsection{Large scale isolation of human mitochondria}

Cells were harvested in PBS and pelleted $15 \mathrm{~min}$ at $4700 \mathrm{rpm}$ and $4{ }^{\circ} \mathrm{C}$. Cell pellets were resuspended in $\mathrm{TH}$ buffer with $0.1 \mathrm{mg} \mathrm{BSA} / \mathrm{ml}$ and pottered $30 \mathrm{x}$ at $800 \mathrm{rpm}$ on ice. Centrifugation steps were carried out in a JA-20 rotor.Cellular debris was pelleted $20 \mathrm{~min}$ at $800 \mathrm{~g}$ and $4{ }^{\circ} \mathrm{C}$. The supernatant 
(SN1) containing mitochondria was collected. The cellular debris was resuspended in TH buffer with $0.1 \mathrm{mg} \mathrm{BSA} / \mathrm{ml}$ and pottered again $30 \mathrm{x}$ at $800 \mathrm{rpm}$ on ice and pelleted $20 \mathrm{~min}$ at $800 \mathrm{~g}$ and $4{ }^{\circ} \mathrm{C}$. The pellet was discarded and the supernatant (SN2) was added to SN1 and pelleted 20 min at $1200 \mathrm{~g}$ and $4{ }^{\circ} \mathrm{C}$. The pellet was discarded and the new supernatant (SN3) was collected and pelleted $20 \mathrm{~min}$ at $14000 \mathrm{~g}$ and $4{ }^{\circ} \mathrm{C}$. The new pellet containing mitochondria was washed with $\mathrm{TH}$ buffer without BSA by resuspeinding it and pelleted $10 \mathrm{~min}$ at $14000 \mathrm{~g}$ and $4{ }^{\circ} \mathrm{C}$. The washed mitochondria were resuspended in $\mathrm{TH}$ buffer without BSA and quantified. They were used immediately or stored at $-80^{\circ} \mathrm{C}$.

\subsubsection{Isolation of human mitochondria for import}

Cells were harvested in PBS with a rubber policeman and pelleted at $600 \mathrm{~g}$, $3 \mathrm{~min}$ and room temperature. The pellets were transferred onto ice and resuspended in cold isotonic buffer with $2 \mathrm{mM}$ PMSF with a plastic Pasteur pipette. Cells were pelleted again and resuspended in cold hypotonic buffer with $2 \mathrm{mM} \mathrm{PMSF}$ ( $5 \mathrm{ml} / 1 \mathrm{~g}$ cells) with a plastic Pasteur pipette and incubated for $7 \mathrm{~min}$. Cells were pottered 10x on ice in a glass potter by hand. Cold hypertonic buffer $(1,1 \mathrm{ml} / 1 \mathrm{~g})$ and cold isotonic buffer $+2 \mathrm{mg} / \mathrm{ml} \mathrm{BSA}$ and $2 \mathrm{mM}$ PMSF $(1,1 \mathrm{ml} / 1 \mathrm{~g})$ was added to the suspension. The sample was pelleted10 min at $1000 \mathrm{~g}$ and $4{ }^{\circ} \mathrm{C}$ and the supernatant (SN1) was collected. The supernatant (SN1) was pelleted $10 \mathrm{~min}$ at $1000 \mathrm{~g}$ and $4{ }^{\circ} \mathrm{C}$ again and the new supernatant (SN2) was collected. SN2 was pelleted 10 min at $10000 \mathrm{~g}$ and $4{ }^{\circ} \mathrm{C}$ and the supernatant (SN3) was discarded. The pellets containing the mitochondria were washed with isolation buffer without PMSF/BSA and pelleted $10 \mathrm{~min}$ at $10000 \mathrm{~g}$ and $4{ }^{\circ} \mathrm{C}$. The washed mitochondria were resuspended in isolation buffer without PMSF/BSA and quantified. Mitochondria were used for experiments after $2 \mathrm{~h}$ incubation on ice.

\subsubsection{Isolation of the crude mitochondrial ribosome fraction}

\subsubsection{Isolation via FPLC}

Mitochondriawere solubilized in solubilisation buffer (immunoprecipitation) with $1 \%$ PCC $(10 \mathrm{mg} / \mathrm{ml})$ for $30 \mathrm{~min}$ at $1000 \mathrm{rpm}$ and $4{ }^{\circ} \mathrm{C}$. The sample was 
pelleted $15 \mathrm{~min}$ at $14000 \mathrm{rpm}$ and $4{ }^{\circ} \mathrm{C}$ and the supernatant was collected. $500 \mu \mathrm{l}$ or $5 \mathrm{ml}$ supernatant were loaded onto a Superdex 200 10/300 GL or a HiLoad 16/600 Superdex 200 pg colum respectively with an ÄKTA purifier system (GE Healthcare). The fractions were collected and analyzed by Western blotting and the flowthrough was processed further for cryoEM analysis.

\subsubsection{Isolation via differential centrifugation}

Mitochondria were solubilized in solubilisation buffer (sucrose cushion) with $0.5 \% \mathrm{PCC}(5 \mathrm{mg} / \mathrm{ml})$ for $30 \mathrm{~min}$ at $1000 \mathrm{rpm}$ and $4{ }^{\circ} \mathrm{C}$. The sample was pelleted $15 \mathrm{~min}$ at $14000 \mathrm{rpm}$ and $4{ }^{\circ} \mathrm{C}$ and the supernatant was collected. $4 \mathrm{ml}$ of the supernatant were loaded onto $3.5 \mathrm{ml}$ sucrose cushion and pelleted $15 \mathrm{~h}$ at $148000 \mathrm{~g}$ and $4{ }^{\circ} \mathrm{C}$. The supernatant, the sucrose cushion and the pellet containing the crude ribosome fraction were collected and analyzed by Western blotting. For cryoEM analysis, only the ribosome pellet was processed.

\subsubsection{Immunoisolation of proteins}

\subsubsection{Immunoisolation with endogenous antibodies}

Preparation of column

Protein-A sepharose (PAS) beads were transferred to a Mobicoil spin column (MoBiTec) and the supernatant was removed by $30 \mathrm{~s}$ centrifugation at $100 \mathrm{~g}$ and room temperature. The beads were washed twice with $0.1 \mathrm{M} \mathrm{KPi}, \mathrm{pH} 7.4$ and $200 \mu \mathrm{l}$ antibody serum and $200 \mu \mathrm{l} 0.1 \mathrm{M} \mathrm{KPi}, \mathrm{pH} 7.4$ were added to the beads. After $1 \mathrm{~h}$ incubation at room temperature, the supernatant was removed by $30 \mathrm{~s}$ centrifugation at $100 \mathrm{~g}$. The beads were washed twice with $0.1 \mathrm{M}$ sodium tetraborate and incubated in $0.1 \mathrm{M}$ sodium tetraborate with $5 \mathrm{mg} / \mathrm{ml}$ dimethyl pimelimidate (DMP) for $30 \mathrm{~min}$ at room temperature. The supernatant was removed and the beads were washed twice with $1 \mathrm{M}$ tris $\mathrm{pH} 7.4$ and incubated in $1 \mathrm{M}$ tris $\mathrm{pH} 7.4$ for $12 \mathrm{~h}$. The supernatant was removed, the beads were washed with $0.1 \mathrm{M}$ glycine $\mathrm{pH} 2.8$ and incubated for $3 \mathrm{~min}$ at $750 \mathrm{rpm}$ and room temperature in $0.1 \mathrm{M}$ glycine $\mathrm{pH} 2.8$. The 
supernatant was removed and thebeads were washed three times with PBS and stored in PBS with $2 \mathrm{mM}$ sodium azide.

Immunoisolation

Mitochondria were lysed in solubilizaiton buffer (immunoprecipitation) $1 \mu \mathrm{g} / \mu \mathrm{l}$ for $30 \mathrm{~min}$ at $1000 \mathrm{rpm}$ and $4{ }^{\circ} \mathrm{C}$. The sample was pelleted $15 \mathrm{~min}$ at $14000 \mathrm{rpm}$ and $4{ }^{\circ} \mathrm{C}$ and the supernatant was collected. The PAS column with cross-linked antibodies was washed three times with solubilizaiton buffer (immunoprecipitation) and the supernatant was transferred onto the column. After $1 \mathrm{~h}$ incubation at $4{ }^{\circ} \mathrm{C}$, the supernatant was removed and the beads were washed ten times with washing buffer (immunoprecipitation). The bound proteins were eluted by $5 \mathrm{~min}$ incubation in $0.1 \mathrm{M}$ glycine $\mathrm{pH} 2.8$ at $750 \mathrm{rpm}$ and room temperature and the supernatant was collected.

\subsubsection{Immunoisolation with Anti-FLAG M2 agarose beads}

Mitochondria were lysed in solubilizaiton buffer (immunoprecipitation) $1 \mu \mathrm{g} / \mu \mathrm{l}$ for $30 \mathrm{~min}$ at $1000 \mathrm{rpm}$ and $4{ }^{\circ} \mathrm{C}$. The sample was pelleted $15 \mathrm{~min}$ at $14000 \mathrm{rpm}$ and $4{ }^{\circ} \mathrm{C}$ and the supernatant was collected. Alternatively, the flowthrough containing the crude mitochondrial ribosome fraction (2.2.4.1) was used. Anti-FLAG M2 agarose beads were transferred transferred to a Mobicoil spin column (MoBiTec) and washed three times with solubilizaiton buffer (immunoprecipitation) and the supernatant was transferred onto the column. After $1 \mathrm{~h}$ incubation at $4{ }^{\circ} \mathrm{C}$, the supernatant was removed and the beads were washed ten times with washing buffer (immunoprecipitation). The bound proteins were eluted by 30 min washing buffer (immunoprecipitation) with $0.4 \mathrm{mg} / \mathrm{ml}$ FLAG peptide (Sigma)at $1000 \mathrm{rpm}$ and $4{ }^{\circ} \mathrm{C}$ and the supernatant was collected.

\subsubsection{Immunoisolation with Anti-FLAG M2 agarose beads from the crude ribosome pellet for cryoEM}

Isolated crude ribosome pellets (2.2.4.2) were lysed in solubilizaiton buffer (immunoprecipitation) $1 \mathrm{\mu g} / \mu \mathrm{l}$ for $30 \mathrm{~min}$ at $1000 \mathrm{rpm}$ and $4{ }^{\circ} \mathrm{C}$. The sample was pelleted $5 \mathrm{~min}$ at $14000 \mathrm{rpm}$ and $4{ }^{\circ} \mathrm{C}$ and the supernatant was collected. Anti-FLAG M2 agarose beads were transferred transferred to a Mobicoil spin column (MoBiTec) and washed three times with solubilizaiton buffer (immunoprecipitation) and the supernatant was transferred onto the column. 
After $1 \mathrm{~h}$ incubation at $4{ }^{\circ} \mathrm{C}$, the supernatant was removed and the beads were washed ten times with washing buffer (cryoEM). The bound proteins were eluted by 30 min washing buffer (cryoEM) with $0.8 \mathrm{mg} / \mathrm{ml}$ FLAG peptide (Sigma) at $1000 \mathrm{rpm}$ and $4{ }^{\circ} \mathrm{C}$ and the supernatant was collected.

\subsubsection{Immunoisolation with Anti-FLAG M2 agarose beads from the crude ribosome pellet for mass spectrometry}

Isolated crude ribosome pellets (2.2.4.2) were lysed in solubilizaiton buffer (immunoprecipitation) $1 \mathrm{\mu g} / \mu \mathrm{l}$ for $30 \mathrm{~min}$ at $1000 \mathrm{rpm}$ and $4{ }^{\circ} \mathrm{C}$. The sample was pelleted $5 \mathrm{~min}$ at $14000 \mathrm{rpm}$ and $4{ }^{\circ} \mathrm{C}$ and the supernatant was collected. Anti-FLAG M2 agarose beads were transferred transferred to a Mobicoil spin column (MoBiTec) and washed three times with solubilizaiton buffer (immunoprecipitation) and the supernatant was transferred onto the column. After $1 \mathrm{~h}$ incubation at $4{ }^{\circ} \mathrm{C}$, the supernatant was removed and the beads were washed ten times with washing buffer (immunoprecipitation). The bound proteins were eluted by $30 \mathrm{~min}$ washing buffer (immunoprecipitation) with $0.8 \mathrm{mg} / \mathrm{ml}$ FLAG peptide (Sigma) at $1000 \mathrm{rpm}$ and $4{ }^{\circ} \mathrm{C}$ and the supernatant was collected. The supernatant was dialyzed against washing buffer (immunoprecipitation) without FLAG peptide and used for mass spectrometry analysis.

\subsubsection{Import of mitochondrial proteins}

Protocolls for protein import modified after (Mick et al., 2012) and protocolls from Alexander Panov (Panov, 2013).

\subsubsection{In vitro synthesis of radiolabeled precursor mitochondria proteins from plasmids}

In vitro synthesis of $\left[{ }^{35} \mathrm{~S}\right] \mathrm{COX} 6 \mathrm{~A}$ from plasmids was carried out with the $\mathrm{TnT}^{\circledR}$ Quick Coupled Transcription/Translation System (Promega). $3 \mu \mathrm{g}$ pOTB7COX6A1 was added to $135 \mu \mathrm{lnnT}{ }^{\circledR}$ Quick Master Mix and the reaction was started with $15 \mu \mathrm{l} 10 \mu \mathrm{Ci} / \mu \mathrm{l}\left[{ }^{35} \mathrm{~S}\right]$ methionine. After $90 \mathrm{~min}$ incubation at $30^{\circ} \mathrm{C}$ and $450 \mathrm{rpm}, 15 \mu \mathrm{l}$ of $200 \mathrm{mM}$ non-radiolabeled methionine solution were added to the reaction. After $1 \mathrm{~min}$ incubation at $30^{\circ} \mathrm{C}$, the reaction was transferred on ice. The solution was used for import experiments directly. 


\subsubsection{In vitro synthesis of radiolabeled precursor mitochondrial proteins from $m R N A$}

In vitro synthesis of $\left[{ }^{35}\right.$ S] NDUFB8 from mRNA was carried out with the Flexi ${ }^{\circledR}$ Rabbit Reticulocyte Lysate System (Promega). The mRNA obtained by in vitro transcription was incubated $3 \mathrm{~min}$ at $65^{\circ} \mathrm{C}$ and transferred on ice immediately. To $66 \mu \mathrm{l}$ rabbit reticulocyte lysate, $2 \mu \mathrm{l}$ amino acid mixture without methionine, $4 \mu \mathrm{l} 2.5 \mathrm{M} \mathrm{KCl}$ and $2 \mu \mathrm{g}$ mRNA were added. RNAse free water was added to the mixture up to $90 \mu \mathrm{l}$. The reaction was started with $10 \mu \mathrm{l} 10 \mu \mathrm{Ci} / \mu \mathrm{l}\left[{ }^{35} \mathrm{~S}\right]$ methionine. After $90 \mathrm{~min}$ incubation at $30^{\circ} \mathrm{C}$ and $450 \mathrm{rpm}$, $10 \mu \mathrm{l}$ of $200 \mathrm{mM}$ non-radiolabeled methionine solution were added to the reaction. After $1 \mathrm{~min}$ incubation at $30^{\circ} \mathrm{C}$, the reaction was transferred on ice. The solution was used for import experiments directly.

\subsubsection{Import and assembly of precursor mitochondrial proteins}

$5 \mathrm{mM}$ ATP were added to the import buffer before the start of the experiment. Mitochondrial were solubilized in import buffer with $5 \mathrm{mM}$ ATP and incubated $3 \mathrm{~min}$ at $30^{\circ} \mathrm{C}$ and $450 \mathrm{rpm}$. The import reactions were performed at $30^{\circ} \mathrm{C}$ and $450 \mathrm{rpm}$ and started with the addition of $10 \%(\mathrm{v} / \mathrm{v})$ radiolabeled precursor proteins. Import reactions were stopped by the addition of the AVO solution after different times (45 min maximum). For the $-\psi$ control, AVO was added in the beginning of the import reaction and the reaction was performed $45 \mathrm{~min}$. After the reaction, mitochondria were pelleted 10 min at $4{ }^{\circ} \mathrm{C}$ and $\max$ speed. The supernatant was removed and mitochondria were washed with SEM buffer. Samples were analyzed by BN-PAGE.

\subsubsection{Mass spectrometry analysis of the mitochondrial ribosome associated MITRAC complex}

Mass spectrometry analysis was carried out by Andreas Linden from the lab of Prof. Henning Urlaub (Göttingen). The sample was isolated after (2.2.5.4) and stored at $-20{ }^{\circ} \mathrm{C}$ before analysis. After the removal of detergents in the sample, proteins were cleaned up and digested by trypsin. The peptides were reduced and alkylated and separated by liquid chromatography on a Dionex UltiMate 3000 UHPLC+ focused (Thermo Scientific) with an analytical column (75 $\mu \mathrm{m}$ x 300 mm, ReproSil-Pur 120 C18-AQ, $1.93 \mu \mathrm{m}$, Dr. Maisch GmbH, 
packed in-house) and analyzed in a Orbitrap Fusion Tribrid mass spectrometer (thermo Scientifc). Obtained data were analyzed with MaxQuant (v. 1.6.0.1, Cox 2008, Nat Biotechnol) against the SwissProt/UniProt human database (reviewed from 14.12.16).

\subsubsection{Electron microscopy analysis of the mitochondrial ribosome associated MITRAC complex}

Analysis of the sample by electron microscopy was carried out by Prof. Holger Stark (Göttingen). The sample was isolated after (2.2.5.3) and analyzed immediately. Negative steins samples were prepeared with $2 \%$ uranyl formate and images were recorded with a CM200 FEG transmission electron microscope (Phillips). For cryoEM analysis, the sample was immobilized on grids with an Vitrobot ${ }^{\mathrm{TM}}$ Mark IV (Thermo Scientific) and grids were transferred to a Titan Krios electron microscope (Thermo Scientific) operated at $300 \mathrm{kV}$. A Falcon III camera (Thermo Scientific) was used to record the images with a magnification of $59000 x$, which resulted in a pixel size of $1.16 \AA$.

\subsubsection{Polymerase chain reaction (PCR)}

PCR was performed with the KOD hot start polymerase (Novagen). Amplification conditions were adjusted according to the specifications of the manufacturer and the melting temperatures of the primers used. PCR produced were analyzed by agarose gels.

\subsubsection{DNA isolation from $E$. coli}

Plasmids were isolated from bacteria using the Wizard ${ }^{\circledR}$ Plus SV Minipreps DNA purification System (Promega) according to the specifications of the manufacturer and stored at $-20{ }^{\circ} \mathrm{C}$ in water.

\subsubsection{Quick isolation of human genomic DNA (gDNA)}

The genomic DNA of MITRAC15 $5^{-/}$clones were isolated using the QuickExtract $^{\mathrm{TM}}$ DNA Extraction Solution. $25 \%$ cells of a confluent 24 well 
plate were used for the gDNA extraction. Cells were pelleted in a reaction tube and the supernatant was removed. $50 \mu$ l of the extract solution was added to the cells and cells were vortexed $15 \mathrm{~s}$. Mixture was heated for $6 \mathrm{~min}$ at $65{ }^{\circ} \mathrm{C}$ and vortexed again $15 \mathrm{~s}$. Mixture was heated for $2 \mathrm{~min}$ at $98^{\circ} \mathrm{C}$ and stored at $-80{ }^{\circ} \mathrm{C}$ or used directly for PCR.

\subsubsection{Molecular cloning}

Molecular cloning procedures were modified after published protocols (Russell, 2000). Fast Digest restriction enzymes (Fermentas) were used for the digestion of plasmids and PCR products according to the specifications of the manufacturer. Ligations were preformed with the DNA Ligation Kit (Thermo Scientific) for 1 hour at $22^{\circ} \mathrm{C}$. Constructs were analyzed by digestion and agarose gel or sequencing.

\subsubsection{DNA electrophoresis and sequencing}

DNA fragments were visualized by agarose gel electrophoresis. Gels with $1 \%$ agarose and $\mathrm{EtBr}$ in TAE buffer were used. Samples were mixed with $10 \%$ FastDigest Green Buffer (10X) (Thermo Scientific) and run in TAE buffer at $100 \mathrm{~V}$ in a Mini-Sub ${ }^{\circledR}$ Cell GT system.GeneRuler DNA Ladder mix (Fermentas) was used as a marker of molecular weight. DNA was visualized using an UVsolo TS transilluminator.

Concentration measurement of the DNA solutions were carried out with the NanoVue spectrometer (GE Healthcare) at 260/280 nm. Wizard ${ }^{\circledR}$ SV Gel and PCR Clean-Up System kits (Promega) were used for the purification of DNA. DNA-sequencing was performed by Seqlab (Göttingen) and data were analyzed with Geneious Pro R11.

\subsubsection{Transformation of $E$. coli}

Competent $E$. Coli cells were generated after the protocol of Hanahan et al. with $\mathrm{RbCl}($ Hanahan, 1983). E. Coli overnight preculture was diluted 1:100 and grown until $O D_{600}=0.3$. Cells were harvested and kept on ice for 15 min and pelleted at $900 \mathrm{~g}$ afterwards. Cell pellet from $200 \mathrm{ml}$ culture was resuspended 
in $67 \mathrm{ml}$ of cold RF1 buffer and suspension was incubated $30 \mathrm{~min}$ on ice. Cells were pelleted and the supernatant removed. Pellet was resuspended in $16 \mathrm{ml}$ of cold RF2 buffer and suspension was incubated $15 \mathrm{~min}$ on ice. Bacteria suspension was distributed in $100 \mu \mathrm{l}$ aliquots, frozen in liquid nitrogen and stored at $-80{ }^{\circ} \mathrm{C}$.

\subsubsection{Gel electrophoresis}

\subsubsection{SDS-PAGE (glycin gels)}

SDS-PAGE protocols with glycin weremodified after the original protocol from Ulrich Karl Lämmli(Laemmli, 1970). Rotiphorese ${ }^{\circledR} \quad$ Gel $30 \quad(30 \%$ Acrylamide/0.8\% bisacrylamide;37,5:1)was used as stock solution to prepare gels. Stacking gels used in this study contained $4 \%$ acrylamide and resolving gels contained $15-17 \%$ acrylamide. Samples were mixed with SDS-loading buffer before loading. Electrophoresis was performed in custom-made gel chambers or Mini-PROTEAN Tetra cell at $30 \mathrm{~mA}, 230 \mathrm{~V}$ per gel.Precision plusPrecision Plus Protein ${ }^{\mathrm{TM}}$ All Blue Prestained Protein Standard was (BioRad) were used as marker.

\subsubsection{SDS-PAGE (tris tricine gels)}

Tris tricine SDS-PAGE gels were modified after the protocol of Hermann Schägger(Schägger, 2006). The Hoefer gel system was used to pour gradient gels. $10-18 \%$ gradients were used in this study as resolving gels and $4 \%$ gels were used for stacking gels. Samples were mixed with SDS-loading buffer before loading. Electrophoresis was performed in custom-made gel chambers or Mini-PROTEAN Tetra cell at $25 \mathrm{~mA}, 80 \mathrm{~V}$ per gel. Precision plusPrecision Plus Protein ${ }^{\mathrm{TM}}$ All Blue Prestained Protein Standard was (BioRad) were used as marker.

\subsubsection{BN-PAGE}

Native complex separation on BN-PAGE was performed after modified protocols from Schägger and von Jagow(Schägger and Jagow, 1991). Mitochondria were solubilized in $\mathrm{BN}$ solubilization buffer (EDTA) and incubater $20 \mathrm{~min}$ on ice. Mixture was pelleted $15 \mathrm{~min}$ at $14000 \mathrm{rpm}$ and $4{ }^{\circ} \mathrm{C}$ and the supernatant was collected. The supernatant was mixed with $10 \% \mathrm{BN}$ 
loading buffer (10X) and incubated $5 \mathrm{~min}$ on ice prior to loading. The Hoefer gel system was used to pour gradient gels. $4-13 \%$ or $2.5-10 \%$ gradients were used in this study as resolving gels and $4 \%$ gels were used for stacking gels. BN cathode and anode buffers were used for gel electrophoresis. Electrophoresis was performed at $15 \mathrm{~mA}, 600 \mathrm{~V}$ per gel at $4{ }^{\circ} \mathrm{C}$. After $1 \mathrm{~h}$ running with $0.02 \%$ Coomassie Brilliant Blue G-250 containing BN cathode buffer, the cathode buffer was exchanged with Coomassie free BN cathode buffer.

\subsubsection{Western Blot}

After protein/complex separation on gel systems, proteins were transferred on PVDF membranes (Millipore) by semi-dry blotting(Gallagher et al., 2001).PVDF membranes were activated by methanol and gels, PVDF membranes and Whatman papers (Heinemann Labortechnik) were equilibrated in blotting buffer prior to blotting. Proteins were transferred from SDS gels to the PVDF membrane at $25 \mathrm{~V}, 250 \mathrm{~mA}$ for $2.5 \mathrm{~h}$ and from BN gels to the PVDF membrane at $25 \mathrm{~V}, 400 \mathrm{~mA}$ for $3 \mathrm{~h}$.

\subsubsection{Coomassie staining}

PVDF membranes (Millipore) with immobilized proteins were stained with Coomassie staining solution for 2 min. Stained membranes were washed with Coomassie destaining solution and dried before further processing.

\subsubsection{Immunostaining}

PVDF membranes (Millipore) with immobilized proteinswere activated with methanol after Coomassie staining and washed with TBST buffer.The activated membrane was blocked for $1 \mathrm{~h}$ in TBST with $5 \%$ milk powder at room temperature. After the blocking the membrane was washed three times 10 min with TBST and incubated for $1 \mathrm{~h}$ in primary antibody solution (in blocking buffer) at room temperature. The membrane was washed three times 10 min with TBST and incubated for $1 \mathrm{~h}$ in HRP coupled secondary antibody solution (in blocking buffer) at room temperature. After three additional washing steps after the incubation, the antibodies bound on the protein/complexes were detected upon incubation of the membranes with 
ECL Western Blotting detection reagent (GE-Healthcare) and signals were visualized on medical X-ray films (Foma).

\subsubsection{Determination of protein concentration}

Protein concentration was determined with Bradford analysis after the protocol of Marion Mckinley Bradford(Bradford, 1976). Roti ${ }^{\circledR}$-Quant reagent (Roth) was used for the reaction and bovine IgG (Biorad) was used as a standard for calibration. Samples were measured at $595 \mathrm{~nm}$ with the Eppendorf ${ }^{\circledR}$ BioPhotometer.

\subsubsection{Fast lysate of HEK293 cells}

Cells were harvested with PBS and pelleted $5 \mathrm{~min}$ at $5000 \mathrm{rpm}$ and $4{ }^{\circ} \mathrm{C}$. Cell pellet was resuspended in 100-200 $\mu$ fast lysate buffer and vortexed for $30 \mathrm{~s}$ at room temperature. The suspension was pelleted $3 \mathrm{~min}$ at $5000 \mathrm{rpm}$ and $4{ }^{\circ} \mathrm{C}$ and the supernatant was collected. Protein concentration of the supernatant was determined.

\subsubsection{Determination of complex I and IV activity}

The activities of the mitochondrial complexes I and IV were assessed by the Complex I Enzyme Activity Microplate Assay Kit (abcam) and the Complex IV Human Specific Activity Microplate Assay Kit (abcam) respectively. Cells used for the measurements were harvested on the same day. 


\section{Results}

\subsection{Structural analysis of the MITRAC complex associated with the translating mitochondrial ribosome}

The structure of themitochondrial ribosome has beenstudied extensively by cryo electron microscopy (cryoEM)recently. High resolution data exists forthe isolated human(Amunts et al., 2015), yeast(Desai et al., 2017) and porcine(Greber et al., 2015b) mitochondrial monosome.The mitochondrial ribosome is in direct contact with the inner mitochondrial membrane (IM) and the application of cryo electron tomography has even enabled in situ structural information of the membrane bound human mitochondrial ribosome. One important feature is the large mitochondrial ribosome subunit $\mathrm{mL} 45$, which was identified to mediate the contact between the mitochondrial ribosome and the IM(Englmeier et al., 2017).

This study aims to gather structural information on the mitochondrial ribosome stalled in translation, which has not been invesigated so far, but is essential to understand the translation process within mitochondria. C12ORF62 was identified as one of the earliest MITRAC components that associates with the mitochondrial ribosome during the translation of COX1(Richter-Dennerlein et al., 2016). Therefore, C12ORF62 was used as abait, to isolate the translating mitochondrial ribosome, together with the early stage of the MITRAC complex. To this end, a HEK293-FIp-In ${ }^{T M}$ T-REx ${ }^{T M}$ cell line was used with a pcDNA5/FRT/TO-C12ORF62 ${ }^{\text {FLAG }}$ inserted into the Flp-In site.

\subsubsection{C12ORF62 ${ }^{\text {FLAG }}$ co-purifies with the COX1 translating mitochondrial ribosome}

To gain structural data of the mitochondrial ribosome in association with MITRAC, first the interaction of $\mathrm{C} 12 \mathrm{ORF} 62^{\mathrm{FLAG}}$ with the mitochondrial ribosome was confirmed. Therefore, C12ORF62 ${ }^{\mathrm{FLAG}}$ containing mitochondria were isolated and solubilized witha buffer containing the mild detergent 
digitonin(1\%). $\quad$ C12ORF62 ${ }^{\mathrm{FLAG}}$ and its interaction partners were immunoisolated.Eluates were separated by SDS-PAGE and membranes wereprobed with different antibodies (Figure 17).

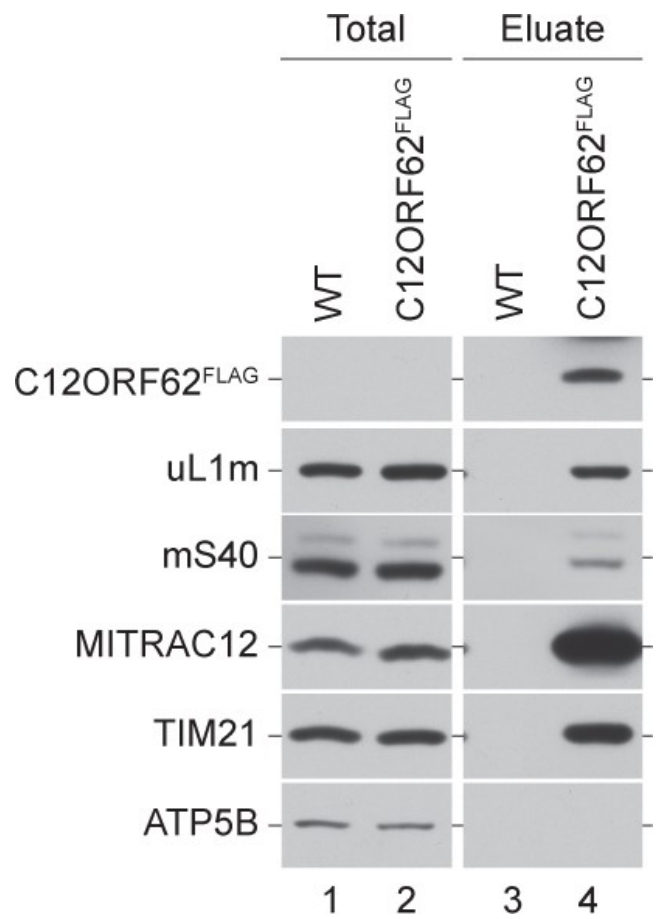

Figure 17C12ORF62 ${ }^{\mathrm{FLAG}}$ copurifies with the large and the small subunit of the mitochondrial ribosome. Mitochondria were isolated from WT andHEK293 Tet-on cells expressingC12ORF62 ${ }^{\mathrm{FLAG}}$ and solubilized with $\mathrm{Mg}^{2+}$ containing buffer with $1 \%$ digitonin as detergent. The immunoisolation was performed via FLAG tag with anti-FLAG agarose beads. $5 \mu \mathrm{g}(0.5 \%)$ of the solubilized mitochondria were loaded as total control and $100 \%$ of the elution were loaded. The sampleswere separated using a $10 \%$ to $18 \%$ tricine gel. The Western blot was probed with antibodies against mitochondrial ribosomal proteins (uL1m: 39S LSU; uS40: 28S SSU) and components from the MITRAC complex (MITRAC12, TIM21).

Besides the expected MITRAC component, MITRAC12, C12ORF62 ${ }^{\text {LAG }}$ also copurified proteins of the small and large mitochondrial ribosomalsubunits, which hinted to an interaction of C12ORF62 with the mitochondrial monosome. A sample of WT mitochondria was used to visualize unspecificallypurified proteins with the anti-FLAG agarose beads. ATP5B, a complex $\vee$ subunit, was probed to show specificity of the experiment.

After confirming the interaction of $\mathrm{C} 12 \mathrm{ORF} 62^{\mathrm{FLAG}}$ with the mitochondrial ribosome, the interaction of $\mathrm{C} 12 \mathrm{ORF} 62^{\mathrm{FLAG}}$ with nascent COX1 was assessed as a characteristic of actively translating ribosomes. To support this interaction, cytosolic translation inHEK293T cells expressing C12ORF62 ${ }^{F L A G}$ was inhibited using emetine and $\left[{ }^{35} \mathrm{~S}\right]$ methionine labelling experiments were performed to visualize mitochondrial translation products. 
Furthermore, puromycin was added to stall translation, resulting in generation of COX1 nascent chain fragments, as described by Richter-Dennerlein et al. (Richter-Dennerlein et al., 2016) (Figure 18).

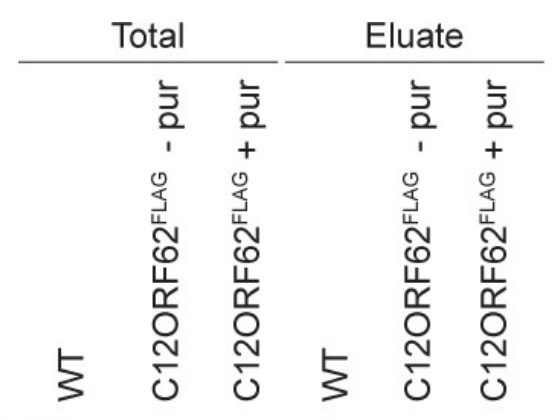

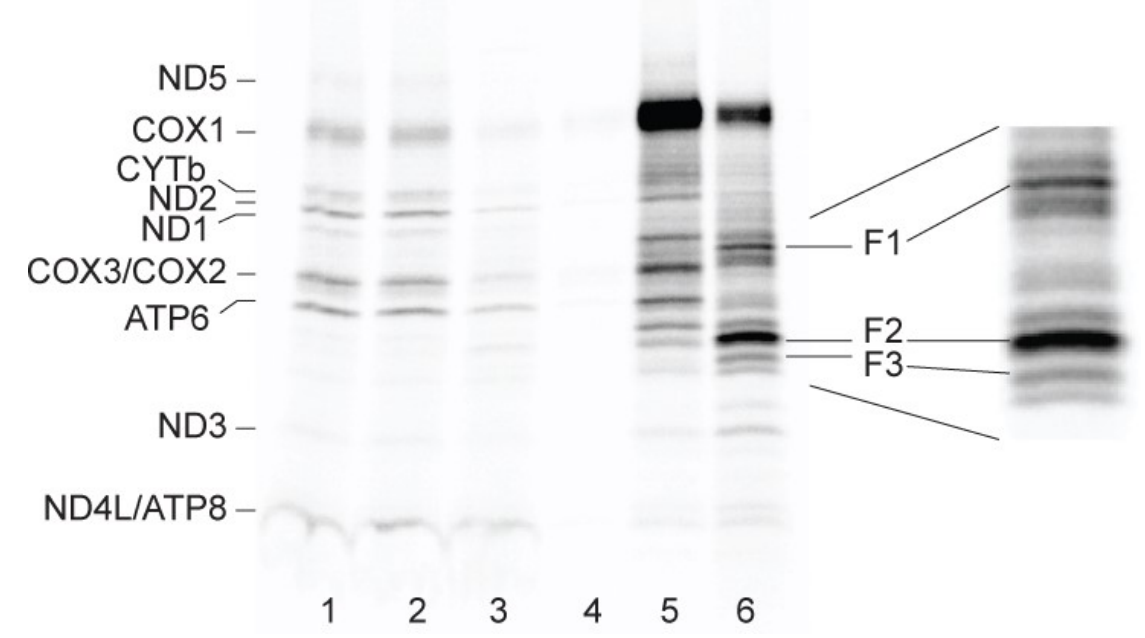

Figure $18 \mathrm{C} 12 \mathrm{ORF} 62^{\mathrm{FLAG}}$ copurifies with fragments of nascentCOX1. $\left[{ }^{35} \mathrm{~S}\right] \mathrm{metabolic}$ labelling was performed inHEK293T-WT and HEK293T-C12ORF62 $2^{\mathrm{FLAG}}$ cells. Puromycin was used to stop the mitochondrial translation and to generate fragments of mitochondrial proteins in translation. Cellswere solubilized in $\mathrm{Mg}^{2+}$ containing buffer with $1 \%$ digitonin as detergent and immunoisolation was performed with anti-FLAG agarose beads. $2.5 \%$ of the solubilized cellswere loaded as total and $100 \%$ of the Elution were loaded. The Fragments F1, F2, F3 are incomplete COX1 fragments released after puromycin treatment. The HEK293T-WT sample serves as a control for unspecific interactions. pur: puromycin

In this experiment, the interaction of C12ORF62 $2^{\mathrm{FLAG}}$ with nascentchains of mitochondrial proteins could be confirmed. The puromycin treated sample displayed an overall weaker mitochondrial-synthesis due to the inhibitory effect of the antibiotic (Figure 18, lane 3). In the eluate, the puromycin treated sample contains less mature COX1, while the signals of the fragments F1, F2 and F3 increased significantly (Figure 18, lane 6). Thosenascentproteinswere described by Richter-Dennerlein et al.as nascent COX1 fragments(RichterDennerlein et al., 2016). 
Based on this, we tried to characterize the mitochondrial ribosome bound complex in association with newly synthesized mitochondrial proteins. Hence, the eluates of $\mathrm{C} 12 \mathrm{ORF} 62^{\mathrm{FLAG}}$ after $\left[{ }^{35} \mathrm{~S}\right]$ metabolic labellingwere further analyzedvia blue native PAGE (BN-PAGE). By using mild and non-denaturing elution conditions and a native gel system(2.5-10\%), large molecular weight complexes could be detected on autoradiograms (Figure 19B).

A

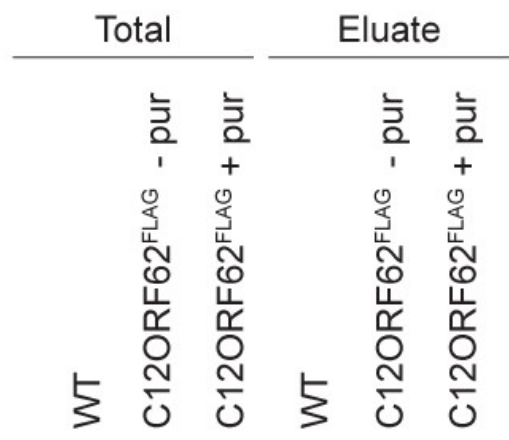

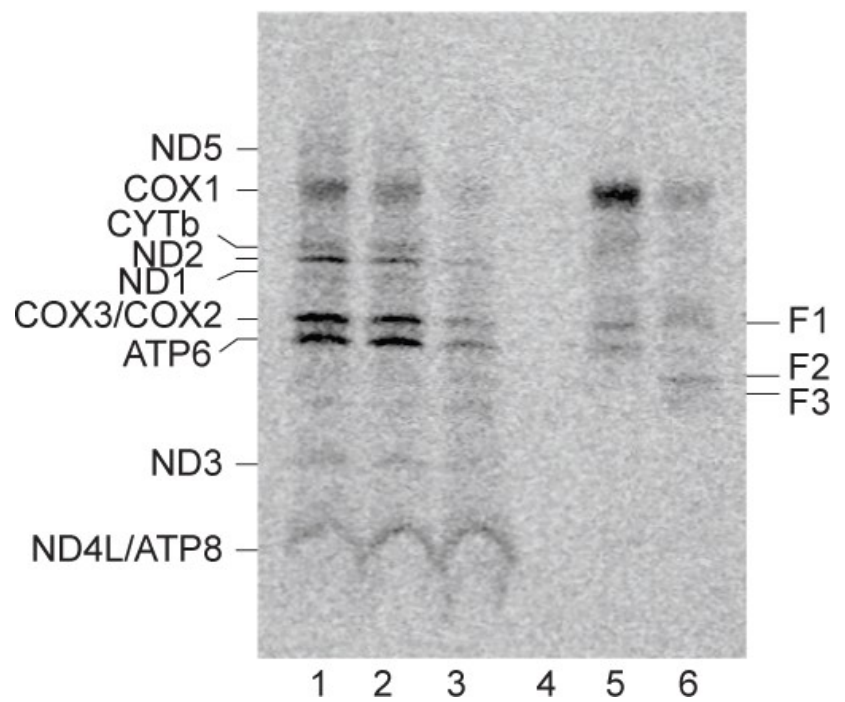

B

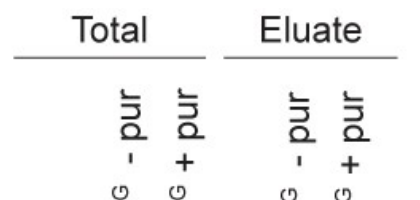

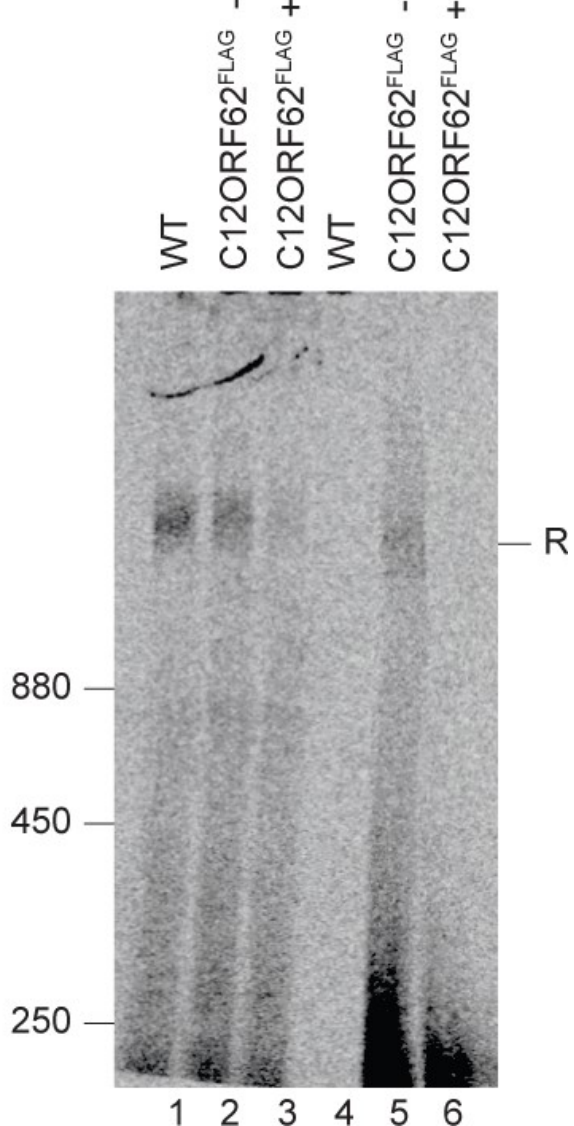

Figure 19C12ORF62 ${ }^{\text {FLAG }}$ coisolated with a high molecular complex containing newly synthesized mitochondrial proteins. $\left[{ }^{35}\right.$ S]Metabolic labelling of newly synthesized mitochondrial proteins was performed similar to thatin Figure 18. Cells were solubilized in $\mathrm{Mg}^{2+}$ containing buffer with $1 \%$ digitonin as detergent and immunoisolation was performed with anti-FLAG agarose beads and bound complexes eluted with FLAG-peptide. A) Denaturing $10 \%$ to $18 \%$ tricine gel as control to verify the effect of puromycin. $10 \%$ elution and $1.875 \%$ solubilized cells were loaded onto the gel. The fragments F1, F2, F3 are nascent COX1 fragments released after puromycin treatment. B) Samples were loaded onto a $2.5 \%$ to $10 \%$ blue native gel. Total: $80 \mu \mathrm{g}$ mitochondrial were solubilized in $\mathrm{Mg}^{2+}$ containing buffer with $1 \%$ digitonin. Eluate: $90 \%$ of the eluate from the isolation was used. A higher complex above $880 \mathrm{kDa}(\mathrm{R})$ containing radioactive signal can be detected. pur: puromycin

Here,a $\left[{ }^{35}\right.$ S]methionine-containing signal in thehigh molecular weightarea of the gelcould be visualized (Figure 19B). Although present in the total control 
of all three samples in this experiment, the signal was of a much weaker intensity inthe puromycintreated sample.Since the size of a full mitochondrial monosome is $2.7 \mathrm{MDa}$ (Amunts et al., 2015), the size of the complex R (Figure $19 \mathrm{~B}$ ) is too small to fit.An explanation would be that the mitochondrial monosome disassociated during the isolation procedure or separation on the $\mathrm{BN}$ gel and the complex $\mathrm{R}$ in fact represents the large ribosomal subunit (1.7 MDa(Brown et al., 2014)), associated with the nascent COX1. Puromycin interferes with mitochondrial translation and causes the release of nascent chains(Azzam and Algranati, 1973), which would lead to a lower amount ofribosomes interacting with nascent COX1, as displayed by thereduced $\left[{ }^{35}\right.$ S]methionine signal (lane 3, Figure 19B). Thus, in the eluates, C12ORF62 ${ }^{F L A G}$ was able to isolate detectable amounts of the higher molecular weight complex without puromycin treatment (lane 5, Figure 19B) and the presence of the antibiotic diminishes the presence of the complex (lane 6, Figure 19B). This supports the conclusion that these complexes werelarge mitochondrial ribosomal subunits with a nascent chain.

Considering the interaction of $\mathrm{C} 12 \mathrm{ORF} 62^{\mathrm{FLAG}}$ with proteins of both the large and small subunits of the mitochondrial ribosome (Figure 17), its copurification with nascent COX1 (Figure 18), and the co-isolation of a puromycin-sensitive higher molecular weight complex (Figure 19), C12ORF62 ${ }^{\text {FLAG }}$ represents an ideal tool for isolations of actively translating mitochondrial ribosomes. Hence, by exploiting the high efficiencies of FLAG immunoisolations, C12ORF62 can be used to purify the translational mitochondrial ribosome-associated stages of the MITRAC complex in significant amounts suitable for structural analysis.

\subsubsection{Defining solubilisation conditionsforthe ribosome-associated MITRAC complex}

In order to generate a sample for structural analysis, two important points have to be considered: 1 . The sample must contain a sufficient amount and concentration of thecomplex to be analyzed. 2. The detergent used to solubilize the complex has to be compatible with the analytical method. While 
detergents are needed to maintain membrane proteins in solution and to counteract their aggregation, they might disturb protein-protein interactions and disassemble complexes. For mitochondrial membrane protein complexes like the mitochondrial translocase(Meisinger et al., 2001) and respiratory chain complexes or supercomplexes(Schägger, 2002;Schägger and Pfeiffer, 2000), digitonin is an established detergent for solubilization.Digitonin is a very mild detergent, which which maintains the integrity of most complexes, but is limited in solubilisation efficiency.

For the isolation of mitochondrial ribosomes, Triton X-100 was used (Schmitt, 1969;Borst and Grivell, 1971).Even thoughTritonX-100 is able to solubilize proteins with high efficiency, it is stringent and therefore not suitable for maintaining the ribosome nascent chain complex. Hence, for the purpose of this aim,it was favourable to use a detergent that has strong solubilizing properties, while maintaining the ribosome-MITRAC interaction. To identify the most suitable detergent,different detergents were testedduring the immunoisolation of $\mathrm{C} 12 \mathrm{ORF} 62^{\mathrm{FLAG}}$. The interaction betweenC12ORF62 ${ }^{F L A G}$ and MITRAC proteins, as well as with mitochondrial ribosomal proteins wasanalyzed(Figure 20). A concentration of $1 \%$ was chosen for the detergents, which are at least $20 x$ the critical micelle concentration (CMC) of the various detergents and therefore provided proper solubilisation properties. A detergent concentration over the $\mathrm{CMC}$ is needed to efficiently solubilize membrane proteins (de Lima Santos H and Ciancaglini, 2000). 


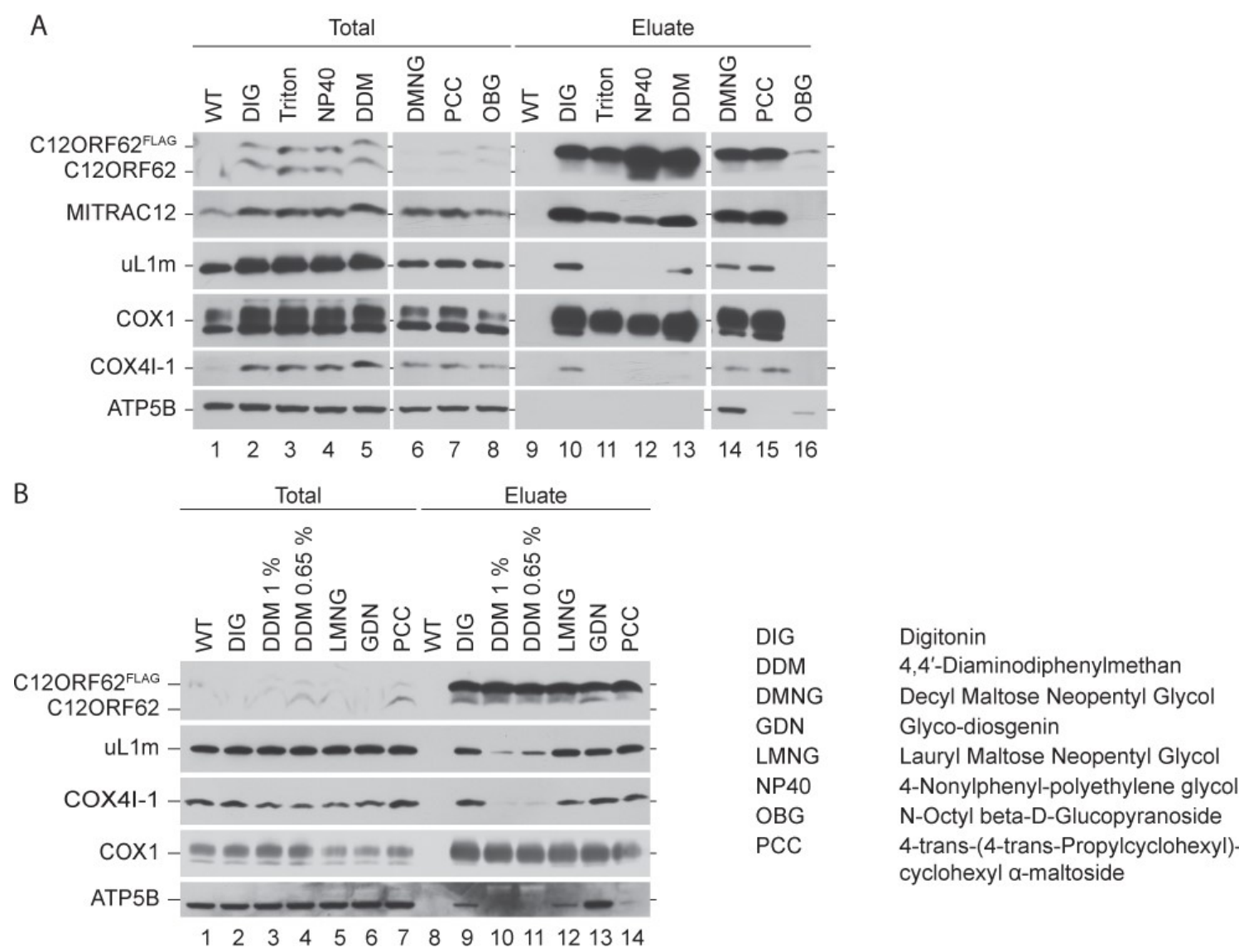

Figure 20PCC provides optimal solubilisation conditions for the isolation of C12ORF62 ${ }^{\text {FLAG }}$ associated complexes. A) and B) HEK293T-WT and HEK293TC12ORF62 $2^{\text {FLA }}$ mitochondria were solubilized in $\mathrm{Mg}^{2+}$ containing buffer with $1 \%$ of different detergents $(0.65 \%$ was tested for DDM). Isolation via agarose anti-FLAG beads were performed to confirm C12ORF62 ${ }^{\mathrm{FLAG}}$ interactions. $1 \%$ total and $100 \%$ of the elution were loaded onto a $10 \%$ to $18 \%$ tricine gel.

Some of the tested detergents, like TritonX-100, DDM (4,4'Diaminodiphenylmethan) or NP40 (4-Nonylphenyl-polyethylene glycol)turned out to be much more stringent than digitonin. In the elution of the immunoisolations performed with these detergents (lane 11, 12, 13, Figure 20A), the signals of MITRAC12, COX1 and COX4I-1were decreased, showing reduced interaction between the bait and the MITRAC complex. The same was true for the interaction between C12ORF62 $2^{\mathrm{FLAG}}$ and the mitochondrial ribosome, since the signal for $\mathrm{uL} 1 \mathrm{~m}$ was also reduced in those lanes. The effect of OBG (N-Octyl beta-D-Glucopyranoside)was even stronger and it disturbed the immunoisolation of the FLAG-tag itself, which means it needed to be tested in a lower concentration (lane 16, Figure 20A). Detergents like DMNG (Decyl Maltose Neopentyl Glycol), (lane 14, Figure 20A) or the digitonin analog GDN (Glyco-diosgenin) (lane 13, Figure 20B), on the other hand, provided rather mild solubilization conditions similar to digitonin, and did 
not disturb the interactions of C12ORF62 ${ }^{\text {FLAG }}$. However,they causedC12ORF62 $2^{\text {FLAG }}$ to co-purify a recognizable amount of complex $V$ subunits, like ATP5B, which is not an interaction partner of the bait and therefore served as a negative control. A possible explanation for this observation is that weaker detergents lack the ability to solubilize the membrane patches of mitochondria sufficiently. Therefore these detergents were not considered for further studies. LMNG (Lauryl Maltose Neopentyl Glycol) seemed to cause some loss of interaction between C12ORF62 ${ }^{\text {FLAG }}$ and the MITRAC complex subunit COX4I-1, but did not affect the interaction of the bait with the mitochondrial ribosomal protein uL1m (Figure 20B, Lane 12). Of the tested detergents, only PCC (4-trans-(4-trans-Propylcyclohexyl)cyclohexyl a-maltoside) showed mild solubilization conditions that are comparable to digitonin, thereby preserving the analyzed interactions. Also, the negative control ATP5B was largely absent in the elution (Figure 20A, Lane 15).

PCC (Figure 21) is a recently developed maltose based compound, designed to provide mild solubilization conditions, similar to digitonin, while having high solubilization efficiency. PCC was tested successfully for the crystallization of cytochrome $b_{6} f$, as well as for Surface Plasmon Resonance studies and for the interaction of the solubilized receptor pair, Patched / Smoothened,of the Sonic Hedgehog pathway (Hovers et al., 2011). LMNG (Figure 21) also belongs to a group of recently developed detergents with a maltose base and low CMC values (Maltose Neopentyl Glycol / MNG). MNGs were designed as detergents that can be synthesized with low effort to serve for large-scale analysis of membrane proteins. LMNG in particular was shown to have superior membrane protein stabilizing properties in comparison to traditional mild detergents like DDM(Chae et al., 2010). Therefore both detergents seemed promising candidates for the isolation of the mitochondrial ribosomeassociated MITRAC complex.

To compare the solubilization efficiencies of PCC and LMNG, alongside the commonly used DDM and digitonin, isolated HEK293T-WT mitochondria were solubilized in detergent-containing buffer with different detergent concentrations. The remaining non-solubilized fraction was pelleted and further solubilized with SDS. Corresponding amounts of supernatant and 
pellet were loaded onto gels to compare the solubilisation efficiencies of membrane proteins (MITRAC12, COX1), in comparison to a soluble protein (TACO1) (Figure 22).

A

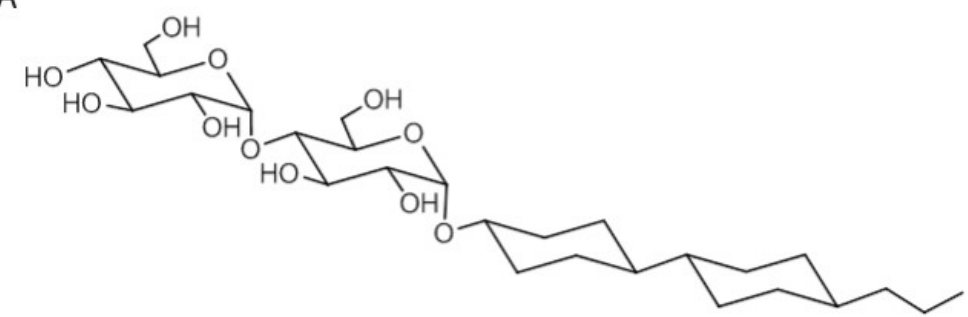

B

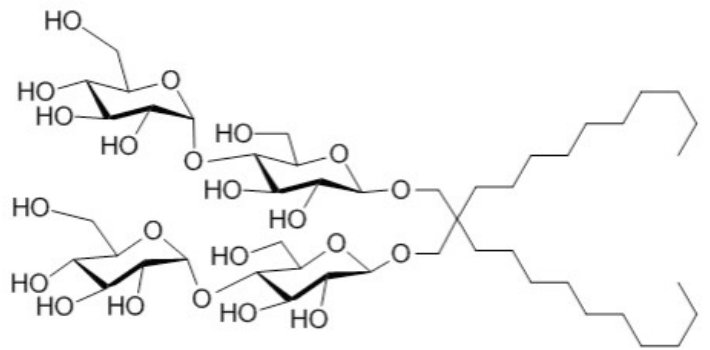

Figure 21 Structure of detergents.A) 4-trans-(4-trans-Propylcyclohexyl)-cyclohexyl amaltoside (PCC- $\alpha$-maltoside) (modified from (Hovers et al., 2011))and B) Lauryl Maltose Neopentyl Glycol (LMNG)(modified from (Chae et al., 2010)).

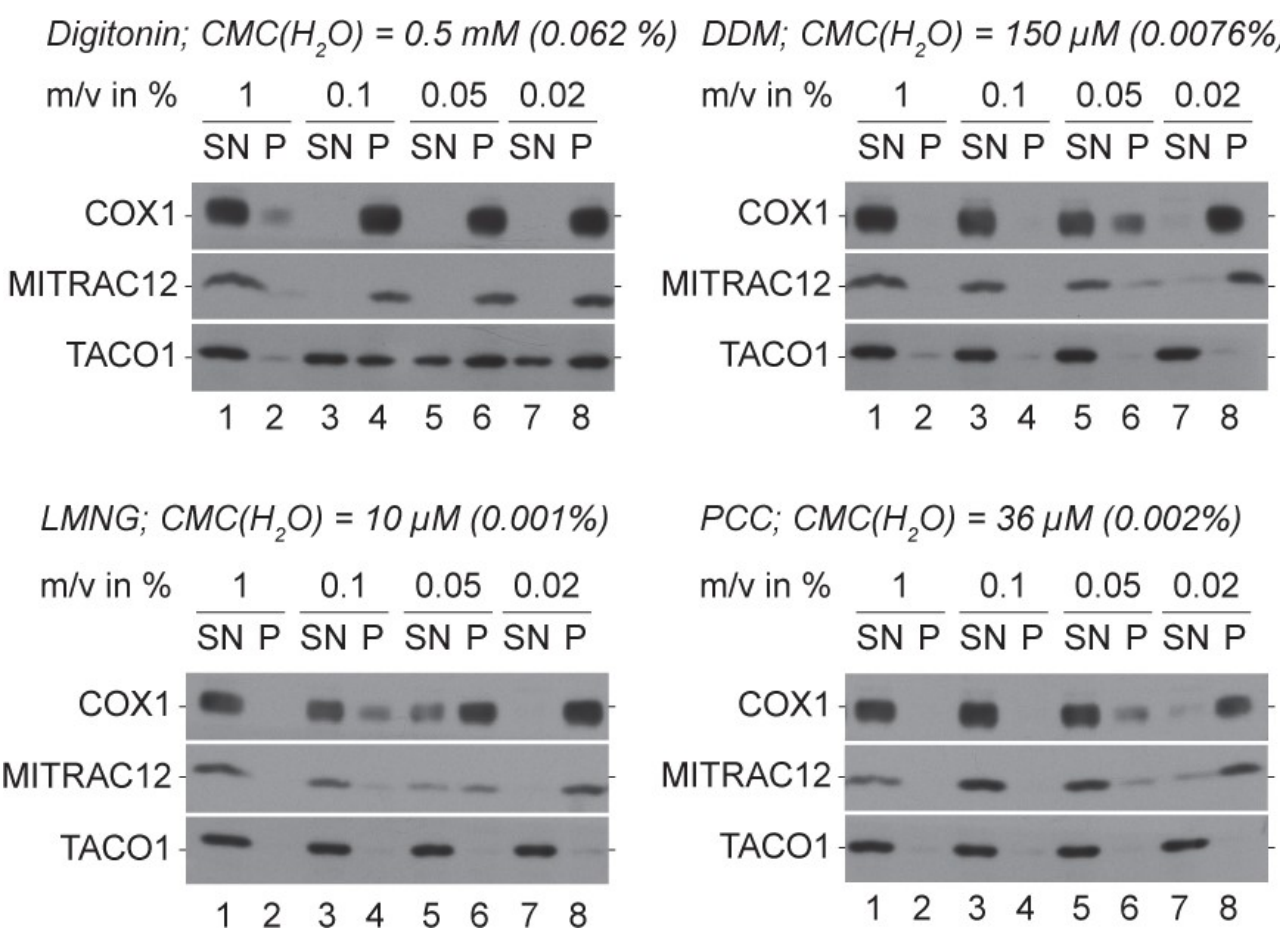

Figure 22PCC provides strong solublizationproperties. HEK293T-WT mitochondria were solubilized in buffer containing different amounts of detergent. Non-solubilized fractions were pelleted for $15 \mathrm{~min}$ at $20817 \mathrm{~g}$ and $4{ }^{\circ} \mathrm{C}$. Pellets were solubilized in a volume of $1 \times$ LD equal to the volume of the supernatant $\left(95^{\circ} \mathrm{C} 5 \mathrm{~min}\right) .20 \mu \mathrm{g}$ supernatant and $20 \mu \mathrm{l}$ solubilized pellet were loaded ontoa10 - $18 \%$ tricine gel. SN: supernatant; P: pellet. 
From the detergent tests it can be concluded that PCC and DDM have the highest solubilisation capacitiesof the tested compounds.0.05\% of PCC or DDM was sufficient to solubilize the membrane proteinsCOX1 and MITRAC12.0.1\% LMNG was requiredfor similar solubilization efficiency. In the case of digitonin, even $1 \%$ was not sufficient to completely solubilize thetested membrane proteins.Considering that $\mathrm{C} 12 \mathrm{ORF} 62^{\mathrm{FLAG}}$ interactions were more stable in PCC in comparison to DDM, PCC was chosen as the detergent to use for the isolation of the mitochondrial ribosome-associated MITRAC complex.

\subsubsection{Separation of different forms of the MITRAC complex}

The MITRAC complex was characterized as an assembly intermediate for the cytochrome coxidase in human mitochondria and is also a dynamic complex with varying composition(Mick et al., 2012;Richter-Dennerlein et al., 2016;Dennerlein et al., 2015).Whileearly MITRAC complex subunits, like C12ORF62, show copurification with proteins of the mitochondrial ribosome, MITRAC subunits which assemble late into the complex, like MITRAC7, do not interact with the mitochondrial ribosome (Richter-Dennerlein et al., 2016), (Mick et al., 2012)(Dennerlein et al., 2015). This leads to the assumption that the interaction between the mitochondrial ribosome and the MITRAC complex only occurs in its earlier stages and that the MITRAC complex disassociates from the mitochondrial ribosome during the progression of complex IV biogenesis. The mitochondrial ribosome-associated MITRAC complex will be referred to as MITRAC ${ }^{\text {ribo }}$ and the free MITRAC complex in its later stages will be referred to as MITRAC ${ }^{\text {free }}$. Using blue native PAGE, the MITRAC complex appears ata size between 140 and $250 \mathrm{kDa}$, which most likely is MITRAC ${ }^{\text {free }}$. The binding of mitochondrial ribosomes to MITRAC increases its size from $230 \mathrm{kDa}$ to over 2.7 MDa(Amunts et al., 2015). This mass shift was utilized to separate MITRAC ${ }^{\text {ribo }}$ from the much smaller MITRAC ${ }^{\text {free }}$. 


\subsubsection{Separation of different forms of the MITRAC complex by size exclusion chromatography}

For the separation of MITRAC ${ }^{\text {ribo }}$ from MITRAC free bysize exclusion chromatography (SEC), thesuperdex 200 size exclusion columns were used. The column material provides a high resolution, in the range between $10 \mathrm{kDa}$ and $600 \mathrm{kDa}$, and has an exclusion size of $1.3 \mathrm{MDa}$. Hence, with a size over 2.7 MDa, MITRAC ribo is expected to be present in the flowthrough. MITRAC ${ }^{\text {free }}$, with a size between 140 and $250 \mathrm{kDa}$, will be retained on the column and therefore separated from MITRAC ribo. By performing FLAG immunoisolation in the flowthrough fraction, MITRAC ${ }^{\text {ribo }}$ can be isolated via C12ORF62 ${ }^{\mathrm{FLAG}}$ (Figure 23).

Isolation of mitochondria

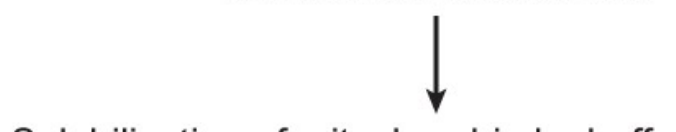

Solubilization of mitochondria by buffer with PCC

Isolation of mitochondrial proteins above $1.3 \mathrm{MDa}$

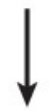

Immunoisolation of MITRAC ribo with C12ORF62 FLAG

Figure 23Isolation strategy for mitochondrial ribosome-associated MITRAC complex via C12ORF62 ${ }^{\mathrm{FLAG}}$. Solubilized mitochondria are subjected to SEC, followed by FLAG immunoisolation. Mitochondrial ribosome containing fractions, present in the flowthrough,are isolated by $\mathrm{C} 12 \mathrm{ORF} 62^{\mathrm{FLAG}}$.

Figure 24shows the chromatogram and Western blot analysis of different fractions of a MITRAC ${ }^{\text {ribo }}$ isolation as described above. 

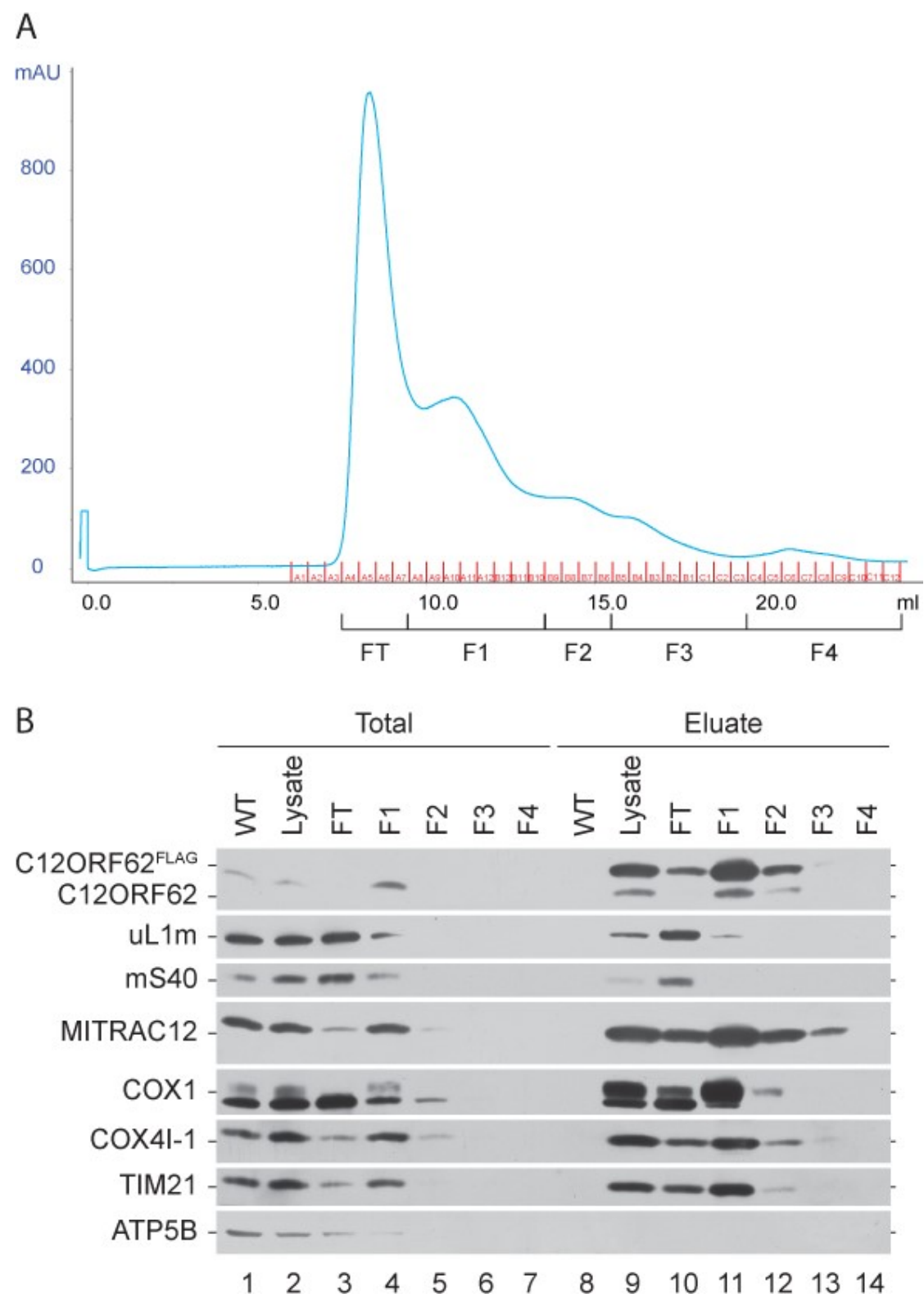

Figure 24 Mitochondrialribosome-associated C12ORF62 ${ }^{\mathrm{FLAG}}$ can be purified bysize exclusion chromatrgraphy (SEC)/immunoisolation tandem experiments. Mitochondria were solubilized in buffer with $1 \%$ PCC. Mitochondrial lysatesweresubjectedto a Superdex 200 10/300 GL column. This was followed by FLAG immunoisolationsfrom collected fractions. FLAG immunoisolations for C12ORF62 $2^{\mathrm{FLAG}}$ was performed from mitochondria lysate as a control (Lysate). $10 \mu \mathrm{g}$ (lysate) or $30 \mu \mathrm{l}$ (FT, F1 - F4) totaland $100 \%$ of the elution were loaded onto a $10 \%$ to $18 \%$ tricine gel. FT: flowthrough; F: fraction.

As presented in Figure 24, usingSEC, large protein complexes like the mitochondrial ribosome could be separated successfully from smaller

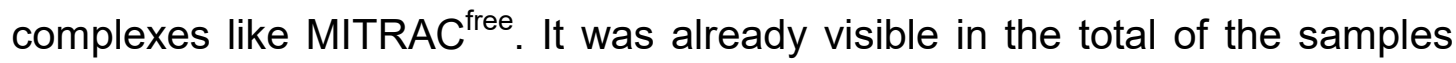
that the ribosomal proteins from both large (uL1m) and small (mS40) subunitswere mainly present in the flowthrough (FT, Figure $24 \mathrm{~B}$, lane 3 ). MITRAC complex subunits (MITRAC12, TIM21, COX1, COX4I-1) were also visible in the $\mathrm{FT}$ sample(Figure $24 \mathrm{~B}$, lane 3 ), but they were predominantly 
represented in the fraction 1 (F1, Figure 24B, lane 4). COX1 and COX4I-1are subunits of complex IV, which has a size of around $400 \mathrm{kDa}$. Therefore, complex IV is able to enter the pores of the gel material and is expected to be present in F1. As already mentioned in 1.1.1, complex IV does not only exist as monomers, but also within respiratory chain supercomplexes with complex I and III. Supercomplexes have a sizeabove 1.3 MDa(Schäfer et al., 2006)and so should be present in the FT. Hence, the signals of COX1 and COX4I-1in the FT could belong to either MITRAC ribo or to respiratory chain supercomplexes.

By comparing the Western blot signals of the eluate from the FT (Figure 24B, lane 10) with the eluate from F1 (Figure 24B, lane 11), it is apparent that theMITRAC complexes isolated by $\mathrm{C} 12 \mathrm{ORF} 62^{\mathrm{FLAG}}$ are different. Compared with the elution from F1, the FT eluate sample (Figure 24B, lane 10) shows a more intense signal for the ribosomal proteins $\mathrm{UL} 1 \mathrm{~m}$ and $\mathrm{mS} 40$ and weaker signals for MITRAC complex proteins (MITRAC12, COX1, COX4I-1, TIM21), as well as the bait C12ORF62 ${ }^{\text {FLAG }}$ (Figure 24B, lane 11). The elution from F1 (Figure 24B, lane 10) shows strong signals for MITRAC ${ }^{\text {free }}$ subunits, while lacking any signals for mitochondrial ribosomal proteins. These resultsconfirmed the hypothesis that SEC, in combination with immunoisolation is a suitable approach to isolate MITRAC ribo independently of MITRAC free.

To determine the amount of MITRAC ribo among the different MITRAC forms, a large scale $\mathrm{C} 12 \mathrm{ORF} 62^{\mathrm{FLAG}}$ was performed from whole mitochondrial lysate in parallel with the purified mitochondrial ribosome fraction. The isolated proteins were separated by SDS-PAGE and analyzed by colloidal coomassie staining and MALDI-TOF mass spectrometry (Figure 25). 


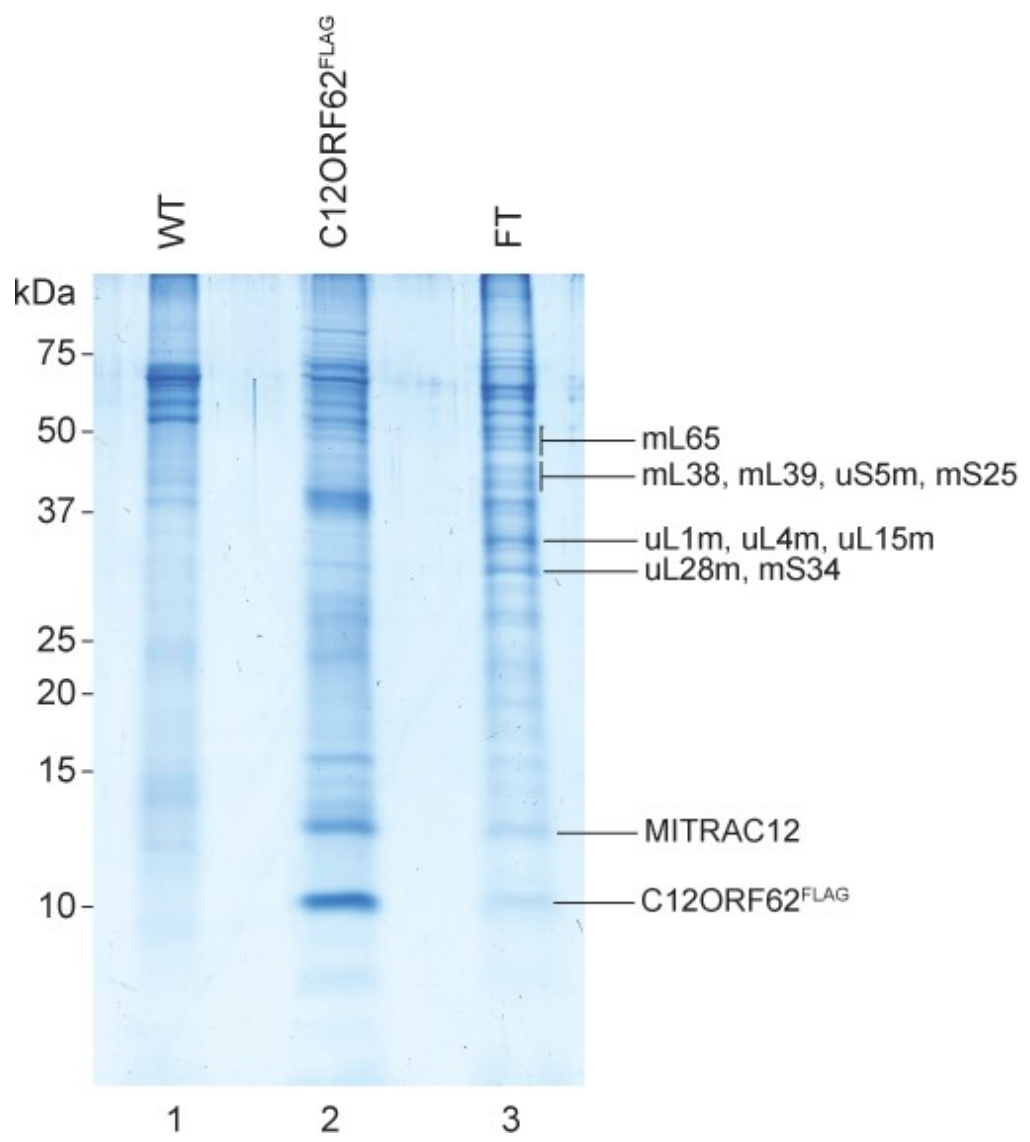

Figure 25Mass spectrometric analysis of MITRAC ${ }^{\text {ribo }}$ isolation via C12ORF62 ${ }^{\mathrm{FLAG}}$ reveals an accumulation of mitochondrial ribosomal proteins.HEK293TC12ORF62 ${ }^{\mathrm{FAG}}$ mitochondria were solubilized with1 \% PCC containing buffer. Mitochondrial ribosome fraction was purified via a superdex 200 high load 16/60 GL column and FLAG immunoisolation was performed in purified fraction(FT). HEK293T-WT (WT) and HEK293TC12ORF62 $2^{\mathrm{FLAG}}$ were solubilized with $1 \%$ PCC containing buffer and FLAG immunopurificationswere performed directly in whole mitochondrial lysate. $100 \%$ of the eluates were loaded onto a $10 \%$ to $18 \%$ tricine gel and analyzed by colloidal coomassie staining and MALDI-TOF mass spectrometry. Bands are labelled with the identified proteins. FT: flowthrough after ÄKTA

Interestingly, the coomassie staining of the elution of the C12ORF62 FLAG isolation from the mitochondrial ribosome fraction (Figure 25, lane 3), showed a different band pattern compared to that isolated from mitochondria lysate (Figure 25, lane 2). According to the staining intensity, the FT sample contained not only much less MITRAC12 and C12ORF62 ${ }^{\text {FLAG }}$, but also severaladditional bands, which were not visible in the $\mathrm{C} 12 \mathrm{ORF} 62^{\mathrm{FLAG}}$ control sample. MALDI-TOF mass spectrometry analysis revealed that most of those bands represented mitochondrial ribosomal proteins. Therefore,MITRAC ${ }^{\text {ribo }}$ was isolated in quantitative amounts by separation from MITRAC ${ }^{\text {free }}$ with SEC.

For gaining structural data, the method of our choicewascryoEM, which is an analytical technique that provides highly precise structural data and is 
established with mitochondrial ribosomes. To determine if a sample is suitable for cryoEM analysis, a large scale isolation was performed. The generated sample was analyzed by negative staining and electron microscopy to assess sample quality. Figure 26 shows a representative picture of the EM analysis.

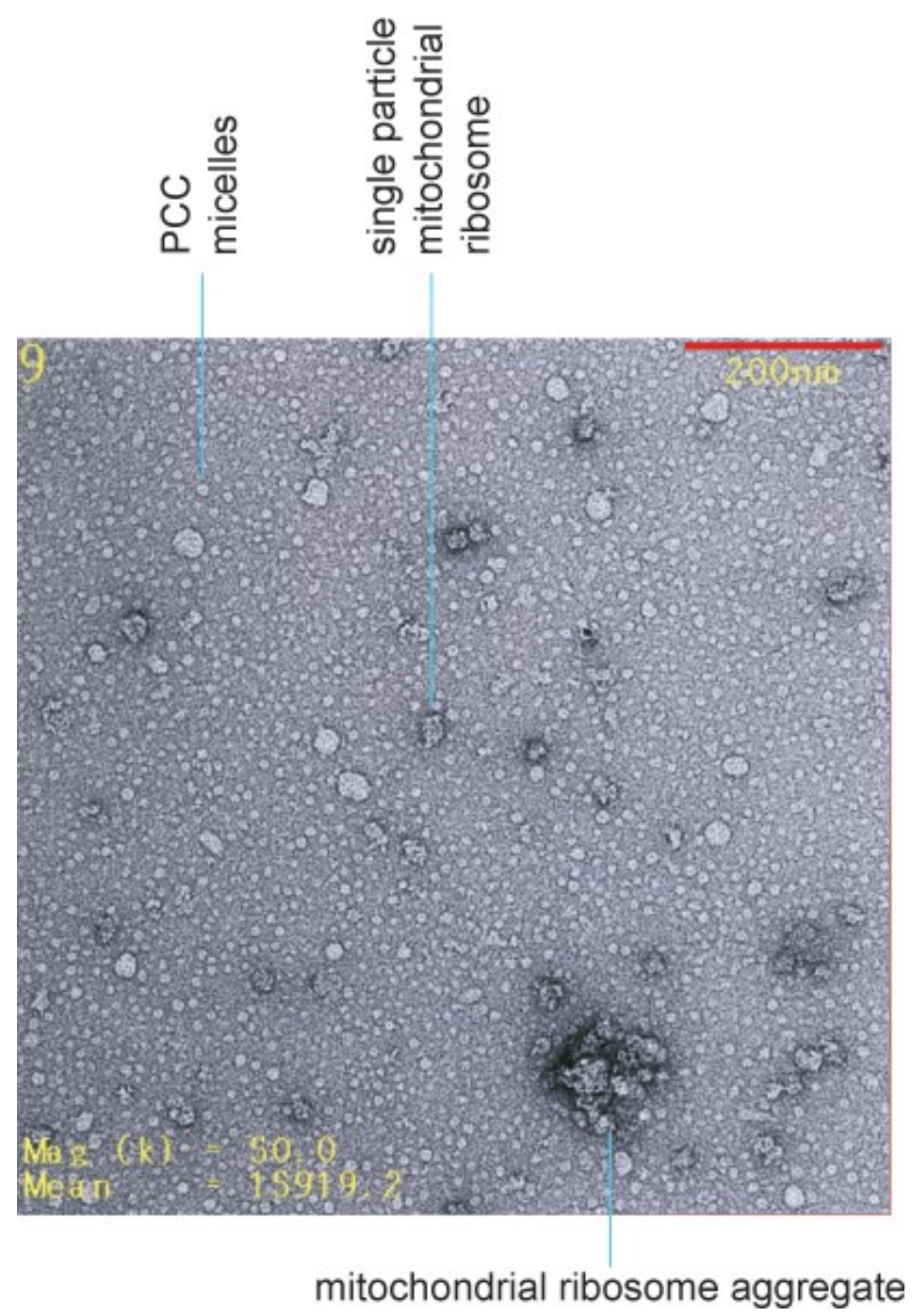

Figure 26Purified MITRAC ${ }^{\text {ribo }}$ shows aggregation under electron microscopy. HEK293TC12ORF62 ${ }^{\text {FLAG }}$ were solubilized with $1 \%$ PCC containing buffer. Mitochondrial ribosome fraction was purified via a superdex 200 high load 16/60 GL column and FLAG immunoisolation was performed in the purified fraction. The sample was treated by negative staining and analyzed by electron microscopy.

The analysis of the negative stained sample revealed that most of the isolated ribosomescould be found in larger mitochondrial ribosome containing complexes, which were most likely mitochondrial ribosomalaggregates. Only a few ribosomes existed as single particles.Single particles are required for cryoEM analysis of complexes. Therefore, the generated sample was not suitable for in-depth structural analysis. The ribosome aggregates were most likely generated during filtration, which was potentially performed under too 
stringent conditions for mitochondrial ribosomes. To overcome this experimental effect, a milder method was required for the purification of the mitochondrial ribosome fraction.

\subsubsection{Separation of different forms of the MITRAC complex via differential centrifugation}

A well established method for the isolation of mitochondrial ribosomes is the use of differential centrifugation. After isolation and solubilisation, the crude ribosome fraction can be obtained as a pellet following a sucrose cushion (34\% sucrose) centrifugation. The pellet contains not only the mitochondrial $55 \mathrm{~S}$ monosome, but also the free $39 \mathrm{~S}$ large and $28 \mathrm{~S}$ small subunits, as well as other larger complexes like the pyruvate dehydrogenase complex (Spremulli, 2007). Different complexes from this sample can be separated further by another centrifugation step on a sucrose gradient (Pfisterer and Buetow, 1981).

For cryoEM analysis a highly concentrated sample was required. Since further sucrose gradient fractionation would lead to a dilution of MITRAC ribo, it was decided to pellet the crude mitochondrial ribosome fraction using the sucrose cushion to keep the sample concentrated. However, because of the small size of MITRAC free (between $140 \mathrm{kDa}$ and $250 \mathrm{kDa}$ ), these complexes will sediment at the surface of the sucrose cushion (Figure 27) and therefore be separated from the mitochondrial ribosome containing pellet. 


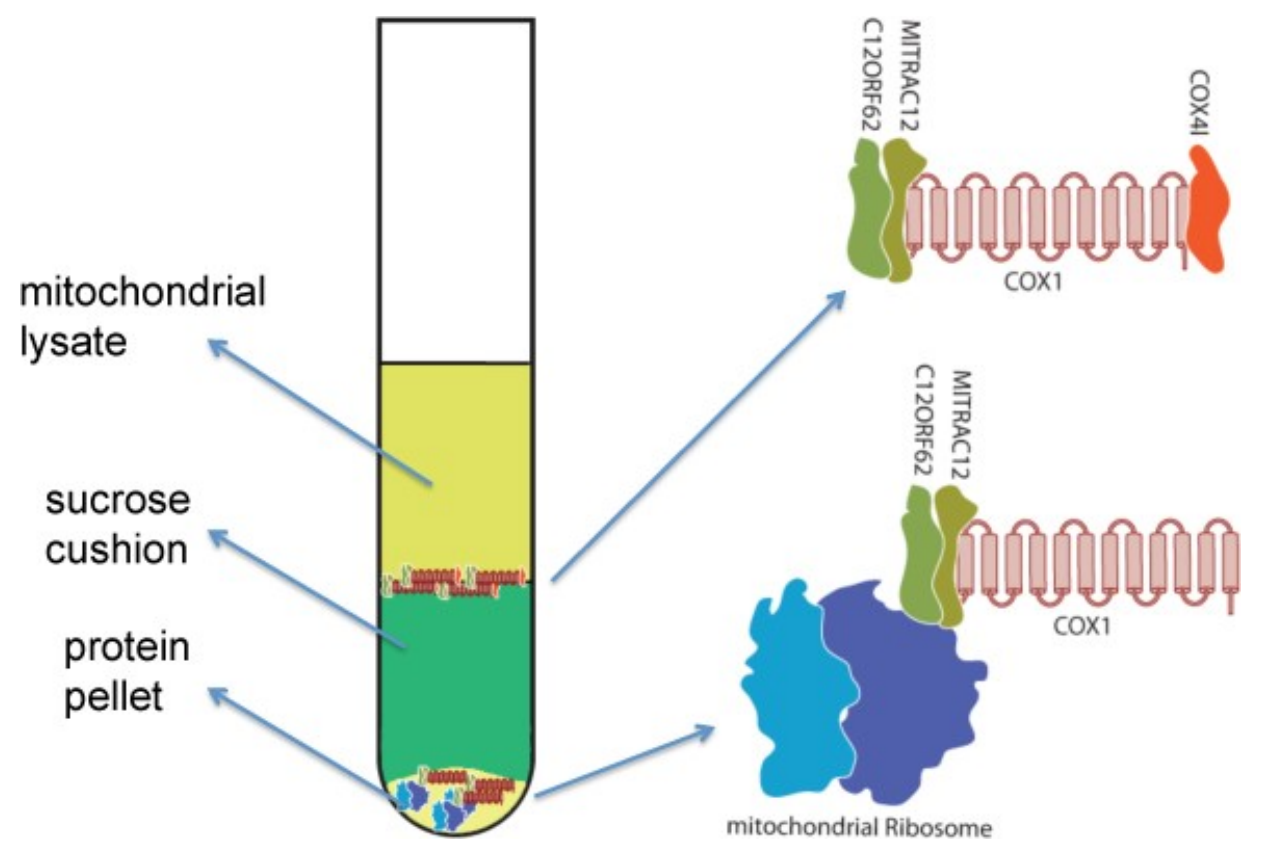

Figure 27 Isolation of the crude mitochondrial ribosome fraction via differential centrifugation.

Since the crude ribosome fraction can be solubilized in a smaller amount of buffer to perform C12ORF62 ${ }^{\text {FLAG }}$ immunopurification, we aimed to increase the concentration of MITRAC ${ }^{\text {ribo }}$ in the elution sample for cryoEM. Therefore, a small scale isolation was performed (Figure 28). C12ORF62 $2^{\mathrm{FLAG}}$ complexes were isolatedfrom each of the three fractions: the remaining supernatant after centrifugation, the sucrose cushion itself and the crude ribosome fraction pellet. 

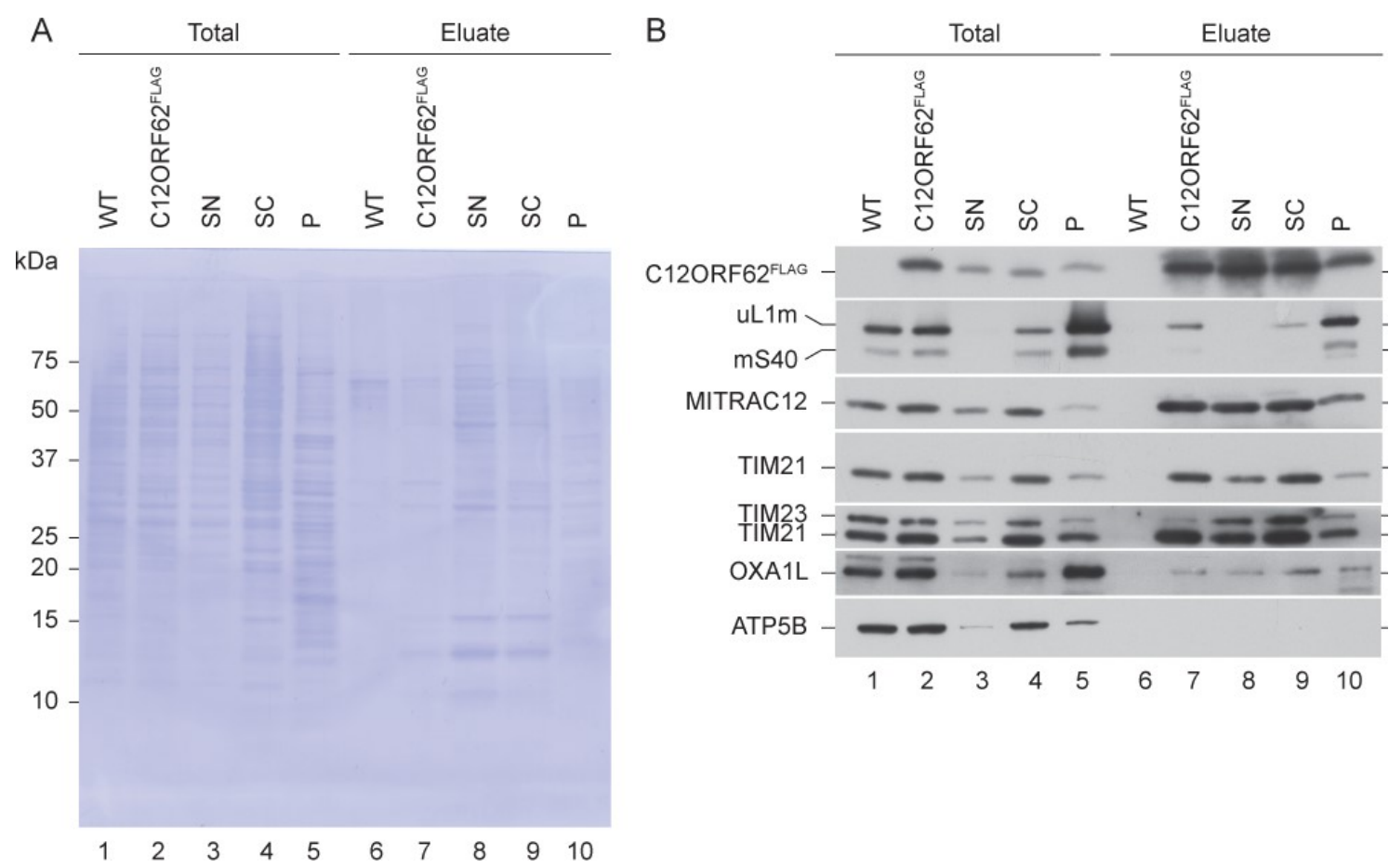

Figure 28Successful isolation of MITRAC ${ }^{\text {ribo }}$ with C12ORF62 $2^{\text {FLAG }}$ via sucrose cushion. HEK293T-C12ORF62 ${ }^{\text {FLAG }}$ mitochondria were solubilized with $0.5 \%$ PCC containing buffer. Mitochondrial lysate was centrifuged for $15 \mathrm{~h}$ at $148000 \mathrm{~g}$. The remaining supernatant (SN) and the sucrose cushion (SC) were isolated. The crude ribosome pellet was solubilized with $0.5 \%$ PCC containing buffer $(\mathrm{P})$. Immunoisolation with anti-FLAG agarose beads was performed for each sample. HEK293T-WT (WT) and HEK293T-C12ORF62 ${ }^{\text {FLAG }}$ were solubilized in $500 \mu \mathrm{l} 1 \%$ PCC containing buffer and FLAG immunoisolation was performed directly in whole mitochondrial lysate. Totals loaded: WT: $10 \mu \mathrm{g} / 1 \%, \mathrm{C} 12 \mathrm{ORF} 62^{\mathrm{FLAG}}: 10 \mu \mathrm{g} /$ $1 \%$, SN: $10 \mu \mathrm{l} / 0,25 \%$, SC: $10 \mu \mathrm{l} /$ 0,29 \% P: $10 \mu \mathrm{l} / 2 \%$. $100 \%$ of the elution was loaded. Samples were loaded onto a $10 \%$ to $18 \%$ tricine gel. A) Coomassie stained membrane. B) Antibodyprobing of membrane.

It was already visible aftercoomassiestaining (Figure 28A), that both the total and eluate of the pellet (Figure 28A lane 5, 10)had a protein composition that differed from the supernatant (Figure 28A lane 3,8), the sucrose cushion (Figure 28A lane 4, 9) and the whole mitochondrial lysate control (Figure 28A lane 2,7). This indicatedthat a separation of proteins or protein complexes by sucrose cushion centrifugation successfully took place.

To verify the separation of MITRAC ${ }^{\text {ribo }}$ and MITRAC ${ }^{\text {free }}$, Western blot analysis was performed and different antibodies were used to probe themembranes (Figure 28B). While the total of the sucrose cushion fraction(Figure 28B lane 4) showed a stronger signal for MITRAC complex subunits like, MITRAC12 or TIM21, in comparison to the pellet fraction (Figure 28B lane 5), the signal for

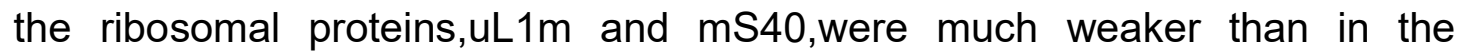
pellet.This indicated a successful accumulation of the crude mitochondrial ribosome in the pellet.The ribosomal proteins present in the sucrose cushion 
fraction might be assembly intermediates of the mitochondrial ribosome, or degradation products, which were too small in size to be pelleted into the crude ribosome pellet. The supernatant fraction showed no signal for ribosomal proteins in both the eluate and total. This indicates that the mitochondrial ribosomal protein subunits mostly enter the sucrose cushion and do not exist as free proteins. The elutionsfrom the pellet sample showeda higher enrichment of ribosomal proteins than the sucrose cushion sample or the whole mitochondria lysate control, but less MITRAC complex subunits. The higher ratio of mitochondrial ribosomal proteinsto MITRAC ${ }^{\text {free }}$ subunits in the pellet samplerepresents a successful isolation of the mitochondrial ribosome-associated MITRAC complex.

Having established the isolation procedure, MITRAC ${ }^{\text {ribo }}$ was purified in large scalefor negative staining and electron microscopy to assess the suitability of the sample for cryoEM. Figure 29 shows a representative electron microscopy picture of the generated sample. 


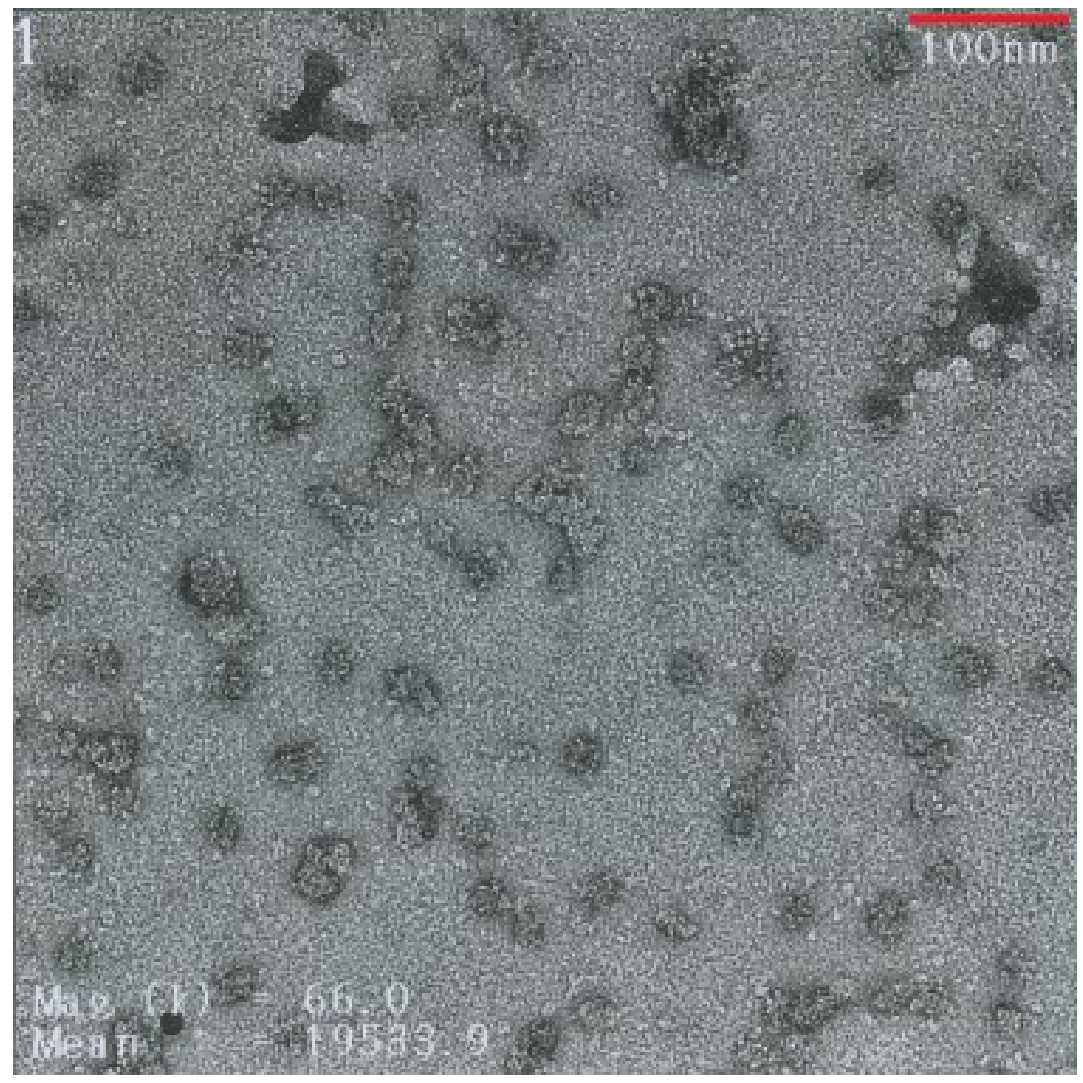

Figure 29Electron microscopy picture of purified mitochondrial ribosome-associated MITRAC complex shows mostly single particles. HEK293T-C12ORF62 ${ }^{\mathrm{FLAG}}$ mitochondria were solubilized with $0.5 \%$ PCC containing buffer. Mitochondrial lysate was centrifuged for $15 \mathrm{~h}$ at $148000 \mathrm{~g}$. The isolated mitochondrial ribosome pellets were solubilized with $0.5 \%$ PCC containing buffer.Immunoisolation with anti-FLAG agarose beads was performed in the resolubilized crude mitochondrial ribosome fraction. Sample was treated by negative staining and analyzed via electron microscopy.

The analyzed sample showed that the isolated complexes mostly exist as single particles, whichwere suitable for cryoEM structural analysis. In conclusion, by using differential centrifugation followed by

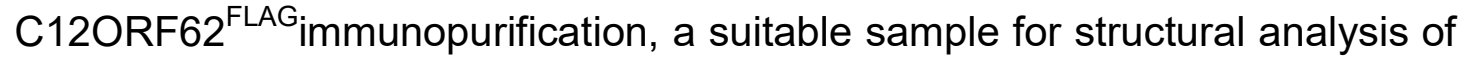
MITRAC ${ }^{\text {ribo }}$ could be generated.

\subsubsection{Mass spectrometric analysis of MITRAC ${ }^{\text {ribo }}$}

To define the composition of MITRAC ${ }^{\text {ribo }}$ in an unbiased manner, theMITRAC ribo complex was isolated from mitochondria and analyzed by mass spectrometry in the lab of Prof. Dr. Henning Urlaub (Göttingen) by Andreas Linden. Peptides were detected for 73 of the 82 known mitochondrial ribosomal proteins. This coverage of $90 \%$ of mitochondrial ribosomal subunits argues in favour of a successful isolation of the mitochondrial 
monosome. A heat map was created for the identified subunits of the mitochondrial ribosome, as well as for subunits and assembly factors of mitochondrial complex IV. The label-free quantification (LFQ) intensity is displayed for the identified proteins or protein groups (Figure 30).

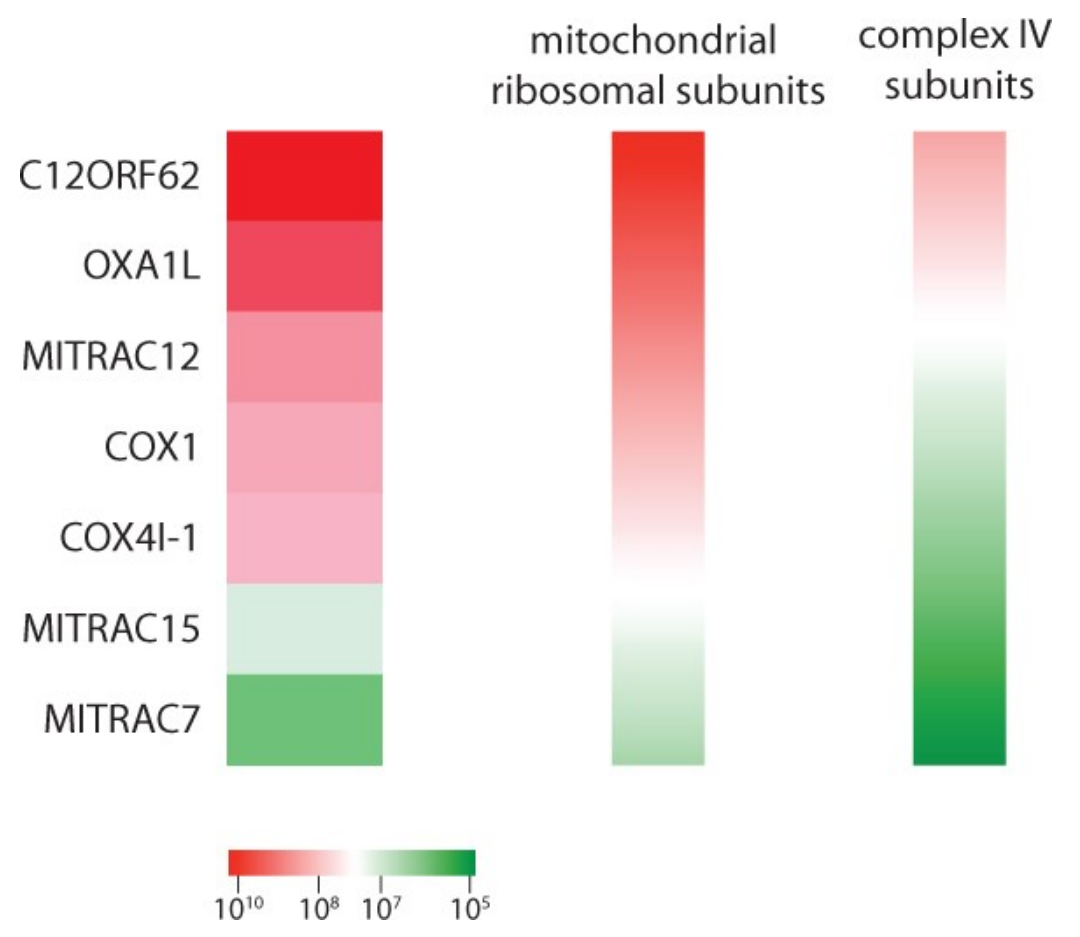

Figure 30 Heat map of mass spectrometry analysis of isolated MITRAC ${ }^{\text {ribo }}$. MITRAC ribo was isolated from $120 \mathrm{mg}$ mitochondria. The isolated complex was analyzed by mass spectrometry. Isolated mitochondrial ribosomal proteins and complex IV subunits and assembly factors are visualized in a heat map, according to their measured LFQ intensities. LFQ intensities correlates to the amount of isolated peptides.

Interestingly, OXA1L, which interacts with MITRAC ribo (Figure 28B) was isolated in high amounts.Considering that OXA1L is the insertase responsible for the transfer of mitochondrial-synthesized proteins into the mitochondrial inner membrane (Wiedemann and Pfanner, 2017), an interaction of OXA1L with MITRAC ${ }^{\text {ribo }}$ was expected. Furthermore, early MITRAC components, like MITRAC12, COX1 and COX4I-1were isolated in moderate amounts, while late MITRAC subunits, like MITRAC15 and MITRAC7, were only purified in very low amounts. In addition, the overall signal intensities of mitochondrial ribosomal subunits were higher than for general complex IV subunits. Hence, the mass spectrometry data verified a successful isolation of MITRAC ${ }^{\text {ribo }}$. 


\subsubsection{Structure of MITRAC ${ }^{\text {ribo }}$}

Although it has been shown to interact with the COX1 nascent chain, the composition and the localization of the mitochondrial ribosome-associated MITRAC is still enigmatic. An assessment of the composition of MITRAC ribo subunits and their interaction to each other, along with their positioning on the mitochondrial ribosome, will provide a structural insight into the mechanisms of COX1 translation and insertion into the inner membrane. To achieve this,we aimed to generate structural data of MITRAC ribo usingcroyEM. Therefore, after the successful purification of MITRAC ${ }^{\text {ribo, }}$, the sample was analysed by cryoEM in the Lab of Prof. Dr. Holger Stark (Göttingen).

The initial step involved the selection of $1,132,000$ particles. After 2D classification, 320,000 particles (28\%) were classified as ribosomal complexes, while the rest were discarded (72\%). The high percentage of unusable particles is rather unusual for the first step of refinement, considering that only $10-15 \%$ of the picked particles are normally discarded after 2D classification. In this case, such a high amount of discarded particles is due to a highly heterogenic sample. The remaining particles underwent 3D classification, which led to the selection of 133,000 particles for the analysis of the large subunit of the mitochondrial ribosome and 83,000 for the analysis of the monosome (Figure 31). These particles were subjected to 3D refinement and post processing, which yielded 3D structures of the large subunit and the monosome with an average resolution of $4.7 \AA$ and $7.4 \AA$ respectively (Figure 32). 
1132000

particles

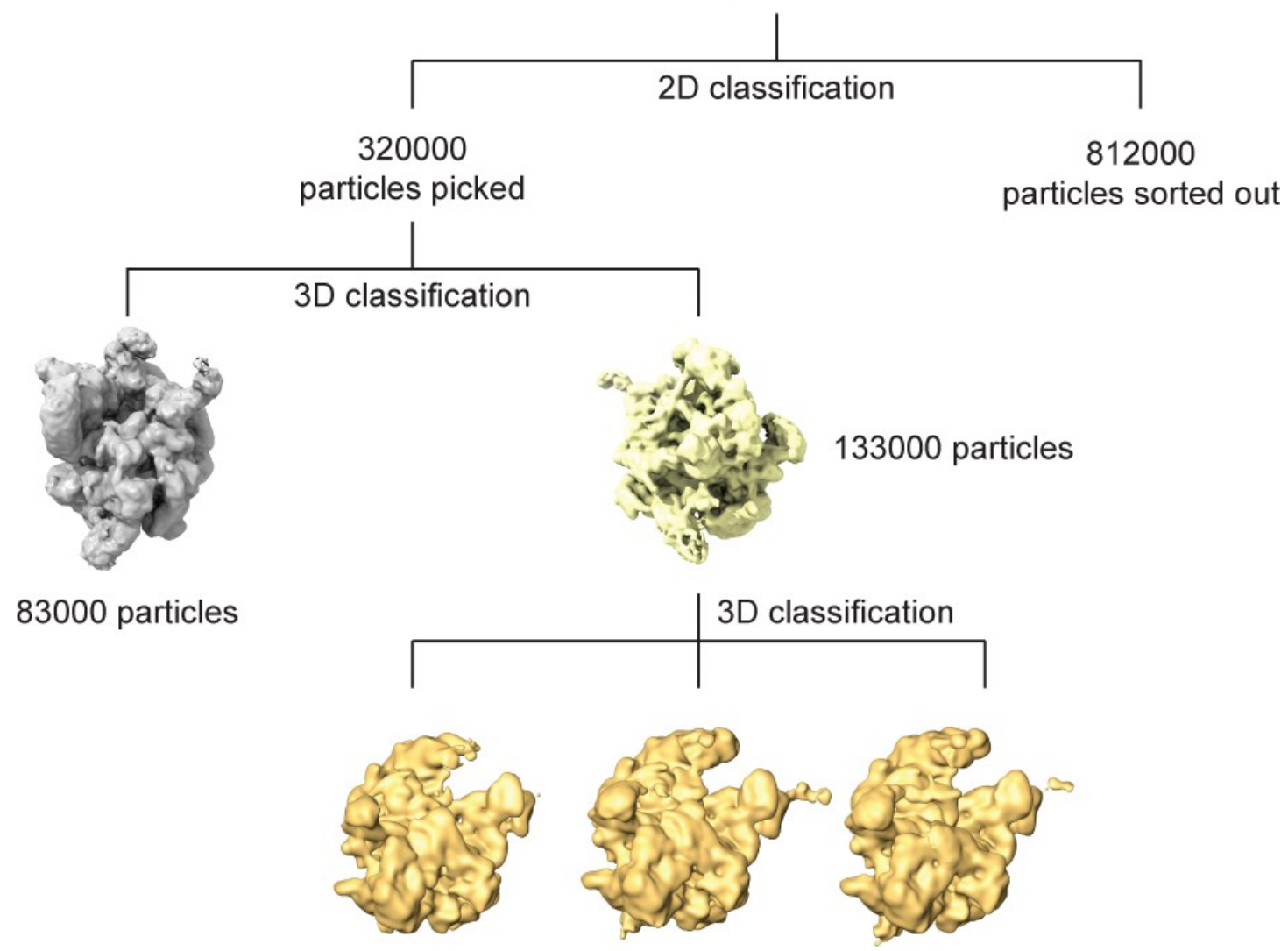

Figure 31 Procedure for the selection of particles for cryoEM analysis of the isolated MITRAC ${ }^{\text {ribo. }}$. 
A

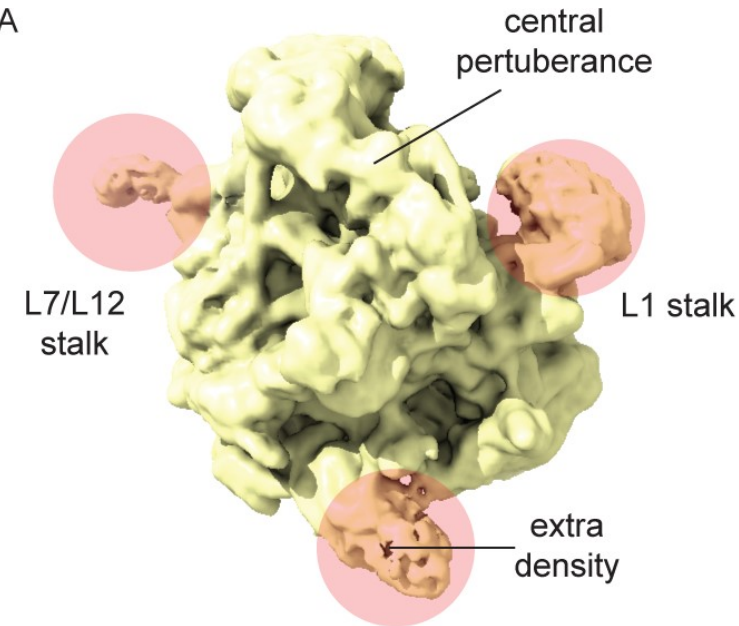

B

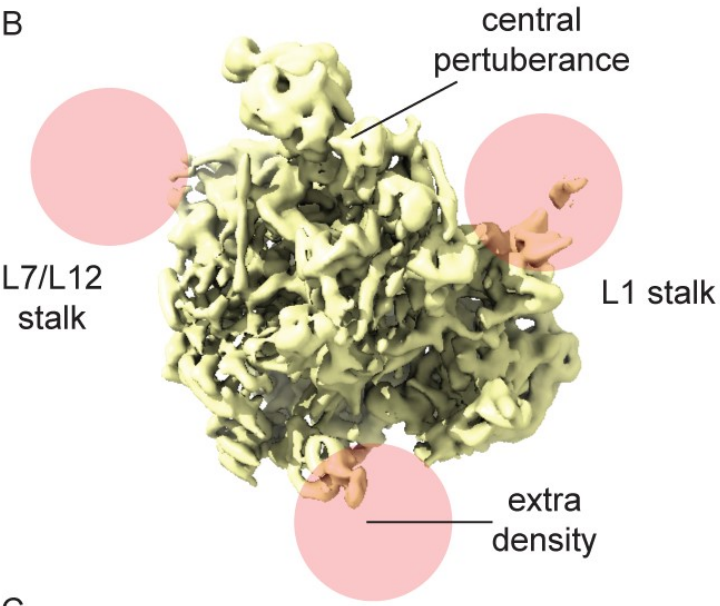

C

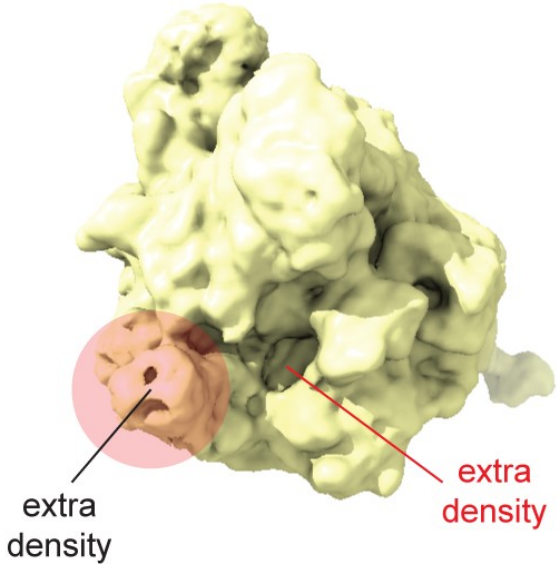

D
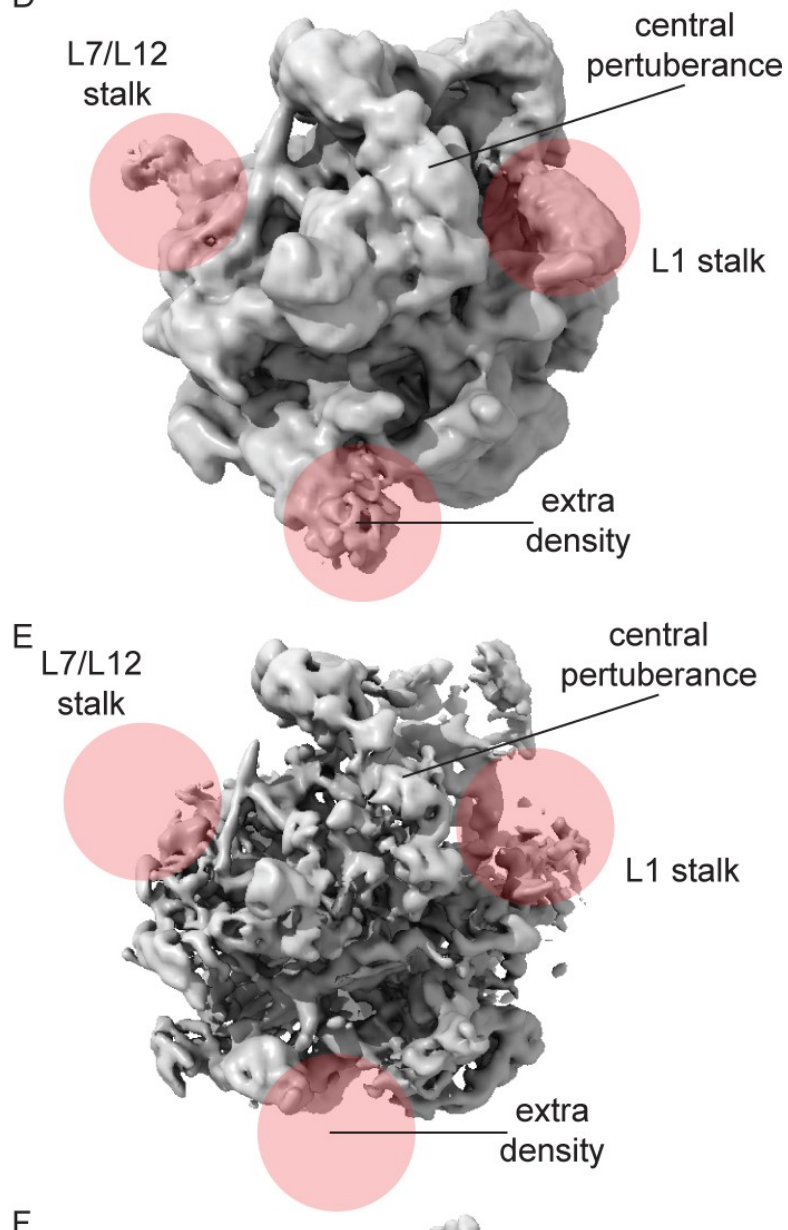

$\mathrm{F}$

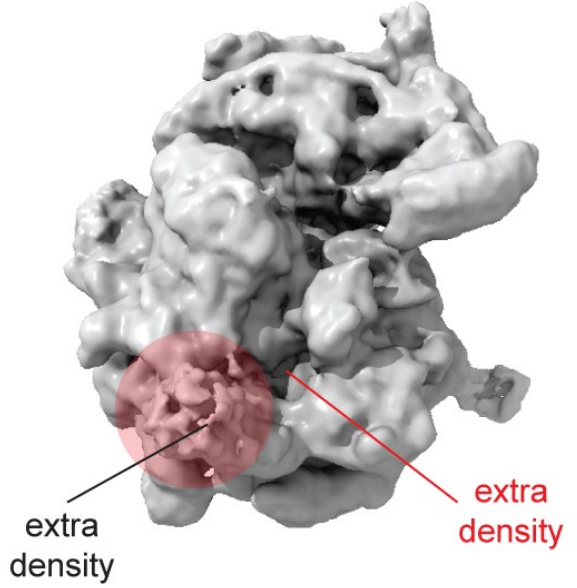

Figure 32 Structure of MITRAC ribo. Structure model of MITRAC ${ }^{\text {ribo }}$ yielded from cryoEM analysis. A), B) and C) show the structure of the free large subunit. The same orientation of the structure is presented in A) low resolution and B) high resolution and the typical ribosomal structural elements are highlighted as well as the extra density. C) shows the position of the extra density relative to the exit tunnel. D), E), andF) show the structure of the monosome. The same orientation of the structure is presented in $\mathrm{D}$ ) low resolution and $\mathrm{E}$ ) high resolution and the typical ribosomal structural elements are highlighted as well as the extra density. C) shows the position of the extra density relative to the exit tunnel.

In both structures, an extra density compared to the known ribosome structurewas visible near the exit tunnel of the ribosome, which most likely represents the MITRAC complex. Even though the averageresolutions for the 
isolated complexes are $4.7 \AA$ and $7.4 \AA$, this mostly because of the high resolution of the mitochondrial ribosome itself. The resolution for the extra density, presumed to be MITRAC, is much lower, suggesting a high flexibility of this moiety. Therefore, an identification of proteins or structural elements within the extra density is impossible with the present dataset. Nevertheless, the133,000 particles selected for the structure of the large subunits were subjected to $3 \mathrm{D}$ classification again, yielding 3 different subsets for the structure of the large subunit, representing different states and conformations of the complex (Figure 33).

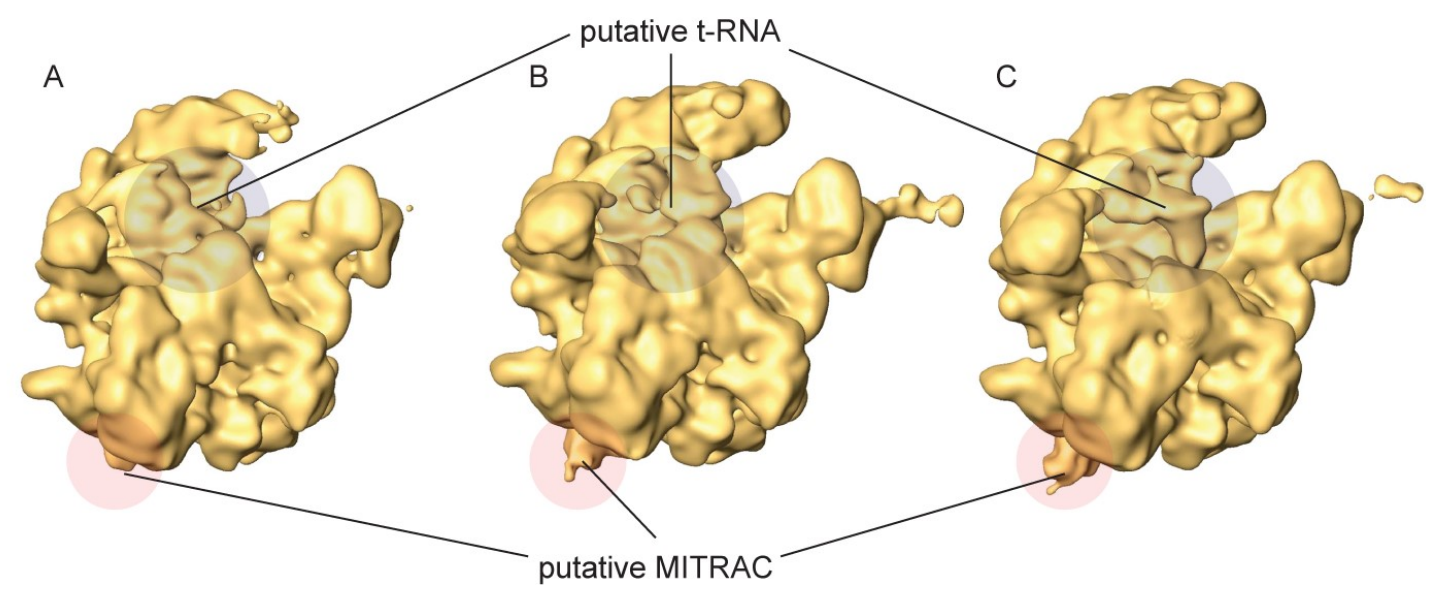

Figure 33 Structure comparison of the subspecies of the isolated large mitochondrial ribosome subunit with MITRAC. The resolution of the putative MITRAC density and the presence and position of the putative t-RNA density vary in the different subspecies.

The 3 different structures have two main differences: 1) the extra density located at the exit tunnel varies in its resolution, which is dependent on its intrinsic flexibility. 2) A structural element located in the active site of the large subunit in the shape of an "L", varies in its orientation in $B$ and $C$, while it is not present in $A$ at all. This " $L$ " shaped structure possibly represents a t-RNA. Thus, the flexibility of the putative MITRAC seems to be dependent on the presence and positioning of the putative t-RNA. 


\subsection{MITRAC15 is an assembly factor that links the biogenesis of mitochondrial complex I and complex IV}

The C7ORF44/MITRAC15 protein has a sequence similarity of $16.2 \%$ (determined with the Needleman-Wunsch algorithm(Needleman and Wunsch, 1970) with the BLOSUM62 matrix (Styczynski et al., 2008) to the yeast assembly factor Coa1, as such, it has been annotated as a homolog of Coa1. In yeast, Coa1 acts downstream of the yeast Cox1 translational activator, Mss51, to regulate Cox1 translation and complex IV assembly. Coa1 has no function in Cox 1 translation, but affects its assembly and therefore stability (Pierrel et al., 2007). Coa1 interacts with Cox14 and Coa3, which are related to C12ORF62 and MITRAC12 respectively. The mechanism of MITRAC15 function has not been investigated until now (Mick et al., 2007;Mick et al., 2010).

MITRAC15 was first described as a MITRAC12 interaction partner by Mick et al. (Mick et al., 2012) and is predicted to have one intermembrane helix. Within this study, MITRAC15 was shown to be a protein integrated into the inner mitochondrial membrane, with its $\mathrm{C}$-terminus facing the transmembrane space. Reduction of MITRAC15 protein levels by siRNA in immortalized fibroblasts was shown to reduce steady state levels of mature complex IV, as well as complex I. Also, MITRAC15 showed interactions with proteins from both complex IV and complex I (Mick et al., 2012).

ACAD9 and ND2 were shown to be interaction partners of MITRAC15. However, the interaction of MITRAC15 with complex IV components, like COX1, or MITRAC subunits, like MITRAC12 or C12ORF62, is rather weak (Richter-Dennerlein et al., 2016; unpublished data, Richarda RichterDennerlein). This result is in agreement with the study published by Guerrero Castillo et al. (Guerrero-Castillo et al., 2017). In this study it was shown that complex I biogenesis involves several different intermediates, which assemble individually and join together for further assembly. One of these assembly intermediates is the ND2 containing $P_{P}$ module. MITRAC15 was shown to be one of the first interaction partners of ND2 in the early assembly intermediate of the $\mathrm{P}_{\mathrm{P}}$ module, which also contains ACAD9. 
These data indicate that MITRAC15 possibly has a dual role in complex IV and complex I assembly.Whether this biochemical process represents two independent processes, or the biogenesis of both complexes coordinated by MITRAC15 at a common nexus, remains enigmatic.

\subsubsection{MITRAC15 is present in different complexes}

A potential involvement in complex I and complex IV biogenesis raises the question of whether MITRAC15 fulfils its role in different complexes, or if the biogenesis of complex I and IV have a common pre-assembly complex with MITRAC15 as subunit. To answer this question, a two-step immunoisolation was performed.MITRAC15 ${ }^{\text {FLAG }}$ was first isolated by FLAGimmunoisolationunder native conditions to isolate all MITRAC $15^{\mathrm{FLAG}}$ containing complexes (Figure 34, lane 4). Afterwards a MITRAC12 immunoisolation was performed, where endogenous MITRAC12 antibodies were immobilized on a protein Asepharose column. The second immunoisolation served toisolate complexes with both MITRAC $15^{\mathrm{FLAG}}$ and MITRAC12as subunits (Figure 34, lane 5). The elutions were analyzed by Western blot. Antibodies were used for proteins of the MITRAC complex (MITRAC12, COX1, COX4I-1) and a protein of the $P_{P}$ module assembly intermediate (ACAD9). 

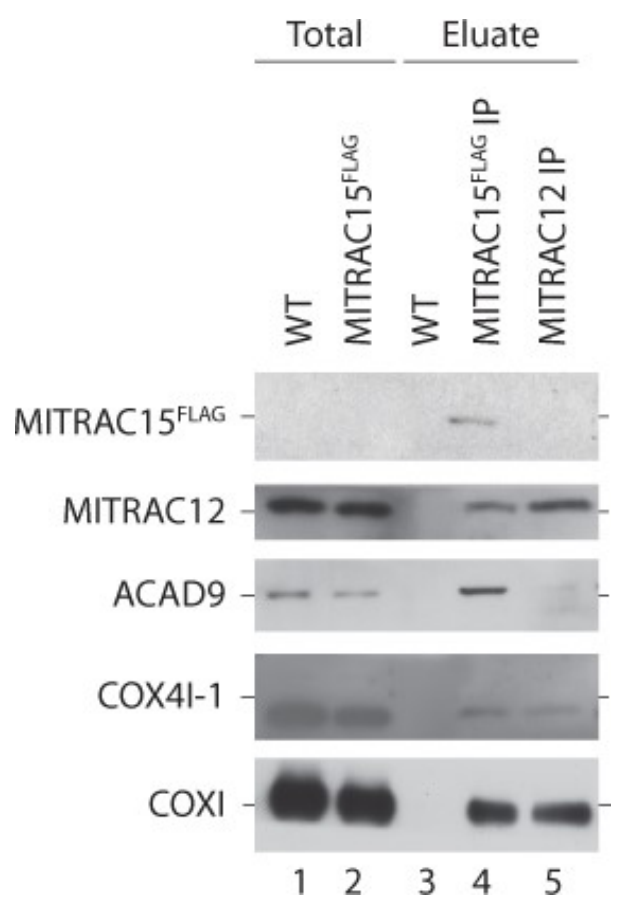

Figure 34MITRAC15 associated MITRAC12 and ACAD9 do not share the same complex. HEK293T-MITRAC15 $5^{\mathrm{FLAG}}$ mitochondria were solubilized in $1 \%$ digitonin containing buffer. FLAG immunoisolation was performed and proteins were eluted with FLAG peptide in their native state. A second immunoisolation was performed in the elution sample with endogenous MITRAC12 antibodies. $10 \%$ of the first elution (MITRAC $15^{\mathrm{FLAG}}$ IP), $100 \%$ of the second elution (MITRAC12 IP) and a wildtype control (WT) were seperated on a $10 \%$ to 18 $\%$ tricine gel and analyzed by Western blot.

As expected, the analysis of the FLAG-immunoisolation revealed that MITRAC15 ${ }^{\mathrm{FLAG}}$ copurifies both complex I and complex IV interaction partners. However, in the elution of the second immunoisolation with aMITRAC12, only MITRAC subunits (COX4I-1, COX1 and MITRAC12)were present. Interestingly, ACAD9 was not copurified with MITRAC12.Thus MITRAC12 and ACAD9 interact with MITRAC15, but not with each other in the presence of MITRAC15. These results indicate that MITRAC15 exists in different independent complexes.

Since ACAD9 and MITRAC12 interact with MITRAC15 in different complexes, we wanted to further characterize those complexes. Therefore, immunoisolations were performed with mitochondria isolated from either MITRAC12 ${ }^{\text {FLAG }}$ or ACAD9 ${ }^{\text {FLAG }}$ expressing HEK293T cells. To properly visualize the complexes, the elutions were first separated on a first dimension on a $4 \%$ to $13 \%$ BN-PAGE gel and then further separated, in a second dimension analysis, using a $10 \%$ to $18 \%$ tricine gel (Figure 35 ). 

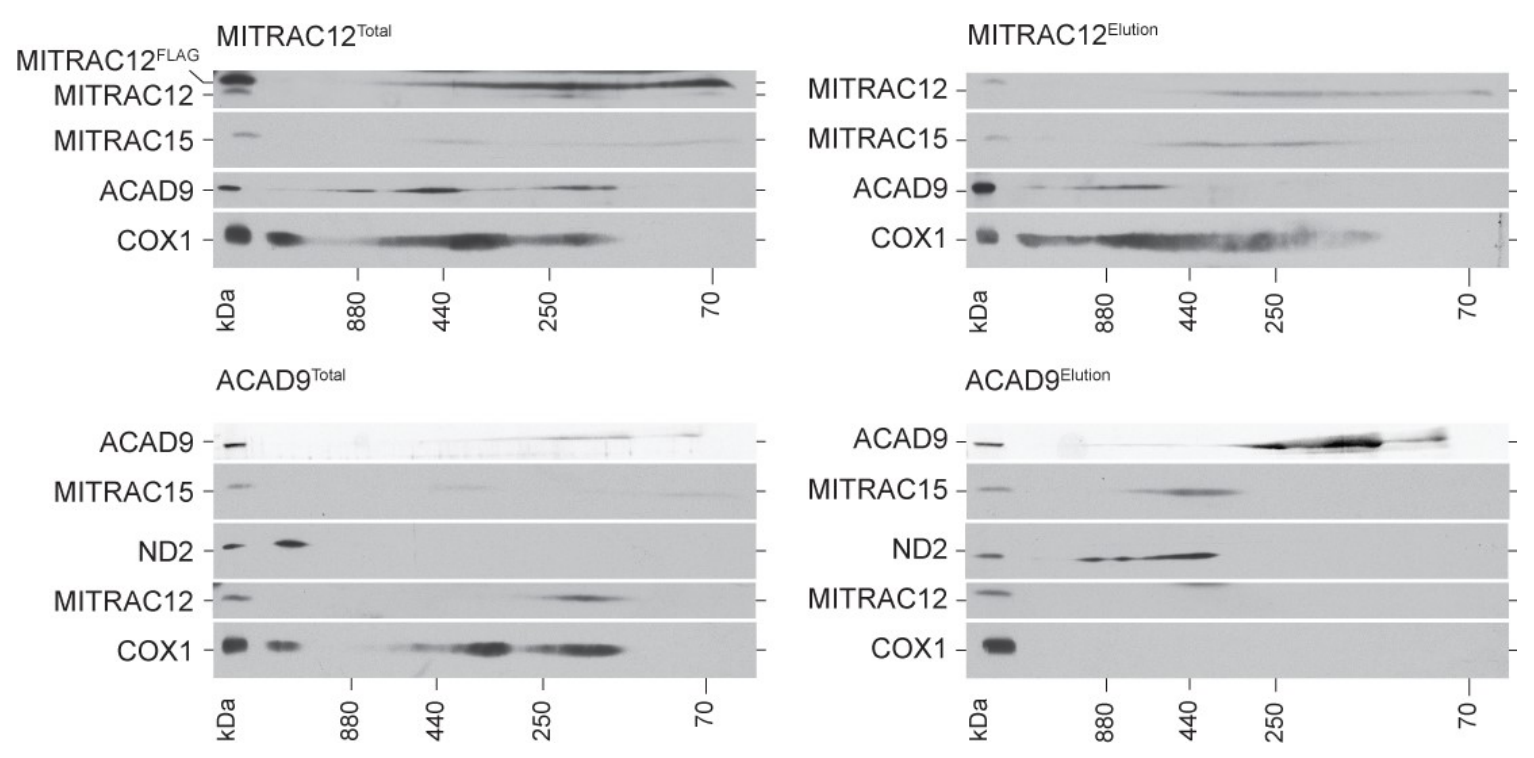

Figure 35MITRAC15 exist in different independent complexes. HEK293T-MITRAC12 $2^{\text {FLAG }}$ and HEK293T-ACAD9 ${ }^{\text {FLAG }}$ mitochondria were solubilized in $1 \%$ digitonin containing buffer. Immunoisolaiton was performed with anti-FLAG agarose beads and eluted under native conditions with FLAG peptide. $2.8 \%$ total and $100 \%$ elution weresubjected onto a $4 \%-13 \%$ blue native gel with a $10 \%$ to $18 \%$ tricine gel in the second dimension.

Analyzing the totals of both samples, it can be observed that MITRAC15 seems to be present in complexes above $250 \mathrm{kDa}$. However, MITRAC15 is only barely present in the MITRAC complex, which runs between $250 \mathrm{kDa}$ and $150 \mathrm{kDa}$. This is in accordance with the data provided by Ricarda RichterDennerlein (Richter-Dennerlein et al., 2016; unpublished data, Richarda Richter-Dennerlein), which shows an interaction of MITRAC15 with MITRAC components, as well as with ND2 and ACAD9. Additionally, there is a smaller complex of unknown composition, around $70 \mathrm{kDa}$, visible for MITRAC15.

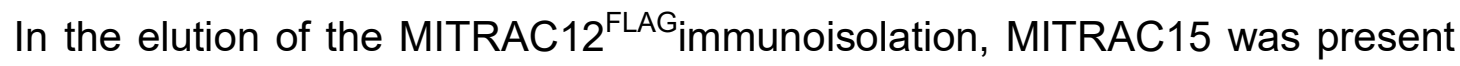
in a complex below $250 \mathrm{kDa}$ and interestingly, also in another complexaround $440 \mathrm{kDa}$. While the smaller complex fits the size of the MITRAC complex, the larger complex,around $440 \mathrm{kDa}$ would fit the size of the $\mathrm{P}_{\mathrm{P}}$ module assembly intermediate(Guerrero-Castillo et al., 2017).Considering that ACAD9 did not comigrate with MITRAC12 in this complex, and MITRAC12 was not reported to be part of an assembly intermediate of the $\mathrm{P}_{\mathrm{P}}$ module, the complex around $440 \mathrm{kDa}$ cannot be defined with the available data. Furthermore MITRAC12 didinteract with ACAD9 in a complex above $440 \mathrm{kDa}$, which is an area where MITRAC12 did not display any MITRAC15 interaction. The interaction between MITRAC12 and ACAD9 was also observed in the isolation of Ricarda Richter-Dennerlein (Richter-Dennerlein et al., 2016; unpublished 
data, Richarda Richter-Dennerlein).Therefore, MITRAC12 interacts with both ACAD9 and MITRAC15 in complexes independent from each other.

In the elution of the ACAD9 ${ }^{F L A G}$ immunoisolation, MITRAC15 only appeared in the complex around $440 \mathrm{kDa}$. ACAD9 also coisolated ND2, which comigrated in the same complex as MITRAC15. Hence, this complex where MITRAC15 and ND2 comigrate should represent an assembly intermediate of the $P_{\mathrm{P}}-\mathrm{b}$ module. This experiment clearly showed that MITRAC15 is associated with at least three different complexes; the putative $P_{P}$ module assembly intermediate (around440 kDa), the MITRAC complex (below $250 \mathrm{kDa}$ ) and another undefined complex at $70 \mathrm{kDa}$.

\subsubsection{MITRAC15 copurifies with newly synthesized mitochondrial proteins}

MITRAC15 is present in both an assembly intermediate for complex IV and in an assembly intermediate for complex I. Since mitochondrial OXPHOS assembly intermediates contain newly synthesized mitochondrial-encoded protein, it can be speculated that MITRAC15 co-isolates mitochondrialencoded core subunits of complex IV or complex I. To investigate this, HEK293T-MITRAC15 ${ }^{\text {FLAG }}$ cells were pulse labelled with $\left[{ }^{35} \mathrm{~S}\right]$ methionine. The assembly of complex I was described as a slow process, up to $24 \mathrm{~h}$ (Guerrero-Castillo et al., 2017) and so labelled cells were chased for $12 \mathrm{~h}$ in $\left[{ }^{35} \mathrm{~S}\right]$ methionine free media to analyze later assembly intermediates with a different protein composition. A FLAG immunoisolation was performed to isolate newly synthesized proteins with MITRAC15 ${ }^{\text {FLAG }}$ (Figure 36). 


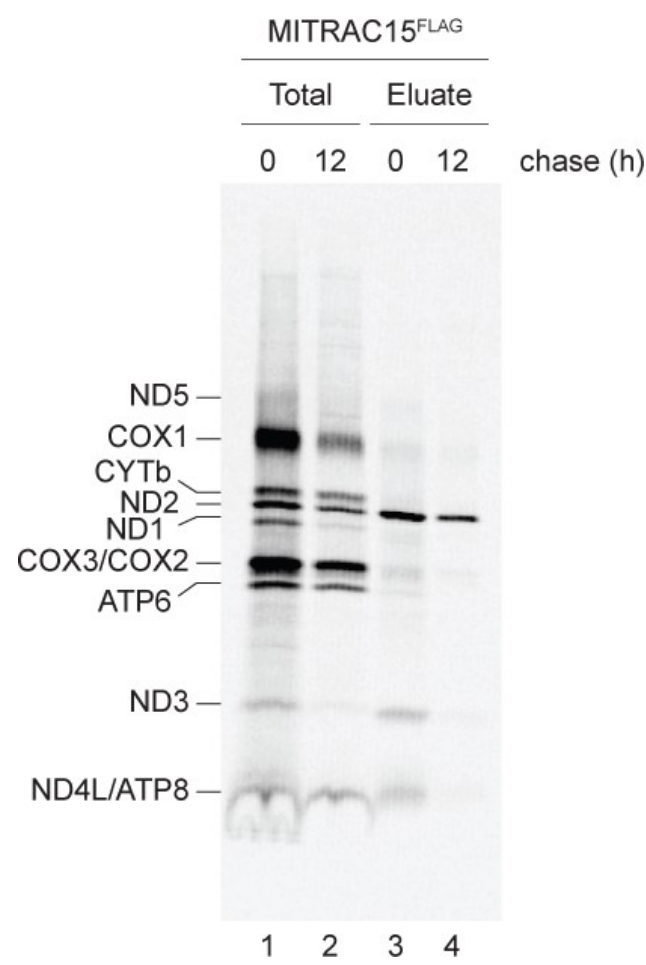

Figure 36MITRAC15 copurifies with newly synthesized mitochondrial proteins. HEK293T-MITRAC $15^{\mathrm{FLAG}}$ cells were labelled for $1 \mathrm{~h}$ with $\left[{ }^{35} \mathrm{~S}\right]$ methionine andeither harvested or further chased for $12 \mathrm{~h}$ in $\left[{ }^{35} \mathrm{~S}\right]$ methionine free media. Cells were solubilized in $1 \%$ digitonin containing buffer. Immunoisolaiton was performed withFLAG agarose beads. $1.1 \%$ total and $100 \%$ elution were subjected on a $10 \%$ to $18 \%$ tricine gel.

This experiment showed that MITRAC $15^{\text {FLAG }}$ is able to coisolate different newly synthesized mitochondrial proteins from complexes I and IV. However, ND2 was particularly enriched in the MITRAC1 $5^{\mathrm{FLAG}}$ eluate. This result is in agreement with published data (Guerrero-Castillo et al., 2017), showing that MITRAC15 is one of the first assembly factors that interacts with ND2. ND3 and ND4L, which are later subunits of the $\mathrm{P}_{\mathrm{P}}-\mathrm{b}$ module (Guerrero-Castillo et al., 2017), could also be coisolated with MITRAC15 $5^{\mathrm{FAG}}$, but to a lesser extent. As expected from previous results of Ricarda Richter-Dennerlein (RichterDennerlein et al., 2016; unpublished data, Ricarda Richter-Dennerlein), MITRAC15 shows only a weak interaction with complex IV subunits such as cOX1 and COX2/COX3.

\subsubsection{MITRAC15 coisolates with fragments of newly synthesized mitochondrial proteins}

MITRAC15 is one of the first interaction partners of newly synthesized ND2 (Guerrero-Castillo et al., 2017) and it interacts with the mitochondrial 
ribosome. It was of interest to determine whether MITRAC15 could copurify nascent chains of mitochondrial-synthesized proteins. To investigate this, $\left[{ }^{35} \mathrm{~S}\right]$ methionine labelling of newly synthesized mitochondrial proteins was performed in HEK293T expressing MITRAC15 $5^{\text {LAAG }}$. After interruption of mitochondrial translation by puromycin treatement, MITRAC $15^{\mathrm{FLAG}}$ was immunoisolated and the elution was seperated on a $10 \%$ to $18 \%$ tricine gel. Proteins were transferred by Western blot and analyzed by autoradiography. Since ACAD9 is a main interaction partner of MITRAC15 and also one of the first subunits in the $P_{\mathrm{P}}$-module assembly intermediate, the same experiment was performed with ACAD9 ${ }^{\text {FLAG }}$ expressing cells (Figure 37).

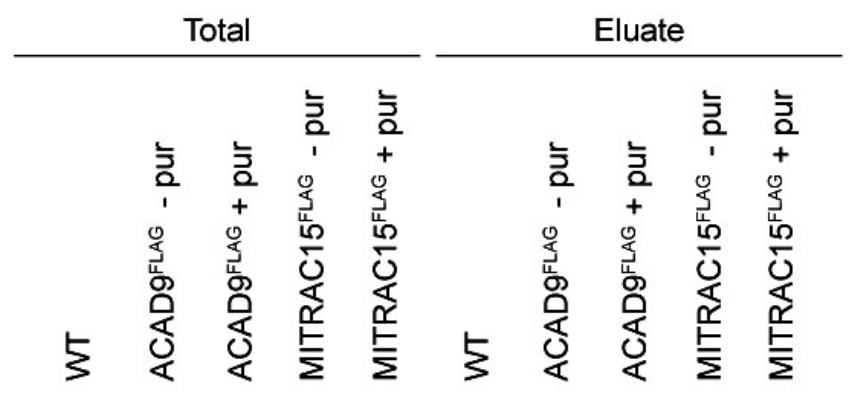

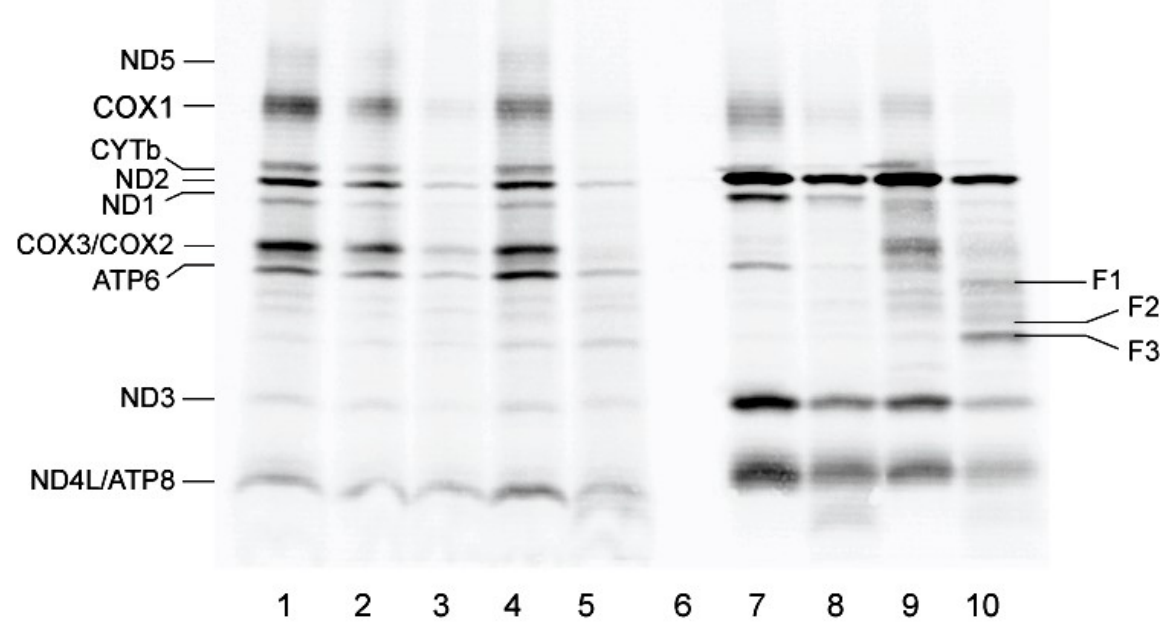

Figure 37 MITRAC15 and not ACAD9coisolates nascent chains of newly synthesized mitochondrial proteins. $\left[{ }^{35} \mathrm{~S}\right]$ methionine labelling of newly synthesized mitochondrial proteins was performed inACAD9 ${ }^{\mathrm{FLAG}}$ or MITRAC15 ${ }^{\mathrm{FLAG}}$ expressing HEK293T cells with and without puromycin treatment. $1.9 \%$ total and $100 \%$ elution were seperated on a $10 \%$ to $18 \%$ tricine gel.

Similar to the experiment performed with C12ORF62 ${ }^{\text {FLAG }}$ (Figure 18), puromycin treatment led to a general decrease in translation. This inference was based on the lower intensity of newly synthesized proteinsin the totals of 
the puromycin treated sample (Figure 37, lane 3,5). A corresponding difference was also observed in the elution, where the puromycin treated samples (Figure 37 , lane 8,10 ) showeda lower amount of coisolated newly synthesized mitochondrial proteins. Interestingly,in the isolation ofMITRAC15 $5^{\mathrm{FLAG}}$ after puromycin treatment(Figure 37, lane 10), three puromycin dependent bandsappeared (Figure 37F1, F2, F3). Thesee uncharacterized fragments may represent nascent chains of mitochondrial proteins stalled in synthesis. Considering that MITRAC15 is one of the first interaction partners of ND2, it is possible that those fragments are ND2 nascent chains.

Unlike MITRAC15, ACAD9 was not able to copurify these fragments (Figure $37 F 1, F 2, F 3)$. Puromycin treatment only led to a generallower amount of newly synthesized mitochondrial proteins that coisolate with ACAD9 (ND2, ND1, COX2/3, ND4L). If the nascent chain fragments belong to ND2, the interaction of MTIRAC15 with ND2 would precede the interaction of ACAD9 with ND2 and make MITRAC15 a candidate for a translation regulator of ND2.

\subsubsection{Knockdown of TIM21 causes accumulation of MITRAC15 at the MITRAC complex}

TIM21 was characterized by Mick at al. 2012 as a shuttle protein that transports complex IV subunits of cellular translational origin from the import machinery to the assembly intermediates. TIM21 could be stalled at the MITRAC complex, or the translocase, after inhibition of cellular or mitochondrial translation respectively. Additionally, TIM21 interacts with newly synthesized mitochondrial proteins of all OXPHOS complexes, suggesting that the TIM21 function extends beyond just complex IV assembly(Mick et al., 2012).

The interaction between MITRAC15 and TIM21 was similar regardless of whether TIM21 was shifted to the respiratory complexes or to the translocase (Mick et al., 2012). This raised the question if TIM21 can influence the shifting of MITRAC15 between its complexes. Therefore, to investigate the role of TIM21 in the complex association of MITRAC15, an siRNA mediated 
knockdown of TIM21 in HEK293T cells expressing MITRAC15 FLAG was performed with following isolation of MITRAC15 ${ }^{\mathrm{FLAG}}$. The eluates were analyzed by Western blotting (Figure 38A). The experiment was also performed with $\left[{ }^{35} \mathrm{~S}\right]$ methionine labelling,prior to FLAG immunoisolation, to adress the effect of a TIM21 knockdown on the interaction of MITRAC15 ${ }^{\text {FLAG }}$ with newly synthesized mitochondrial proteins (Figure 38B).

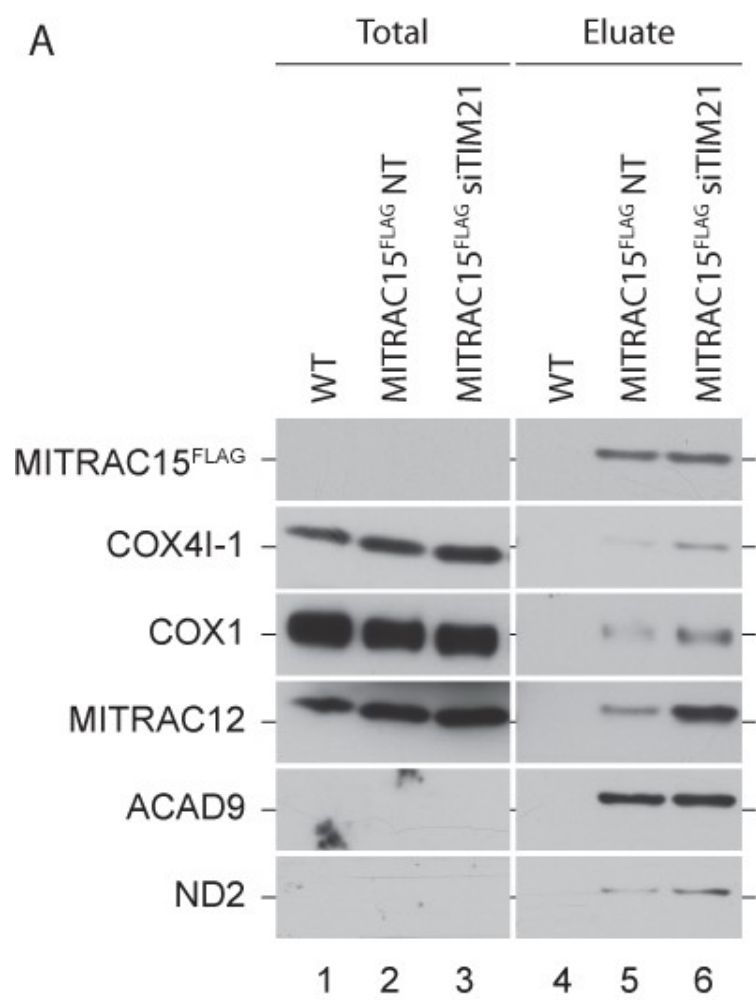

B

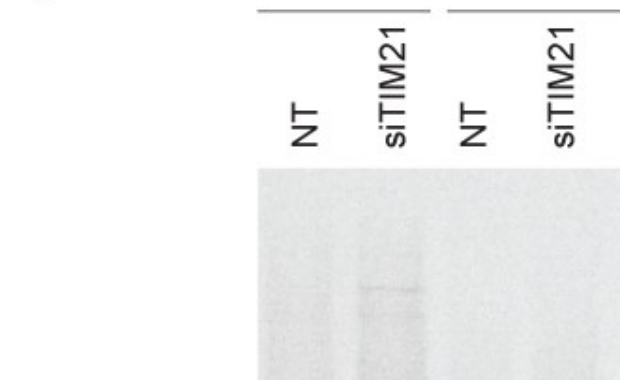

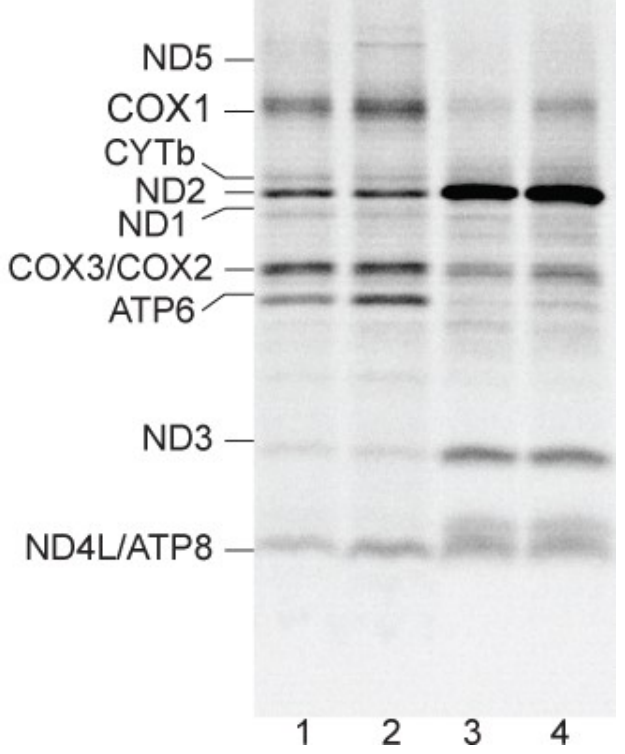

Figure 38Knockdown of TIM21 causes a higher association of MITRAC15 with the MITRAC complex. HEK293T cells expressing MITRAC $15^{\mathrm{FLAG}}$ were labelled $1 \mathrm{~h}$ with $\left.{ }^{35} \mathrm{~S}\right]$ methionine. Cells were solubilized in buffer containing $1 \%$ digitonin. Immunoisolation was performed with FLAG agarose beads. Totals and eluates were separated on a $10 \%$ to $18 \%$ tricine gels. A) Western blot analysis was performed usingthe above mentioned antibodies. $10 \mu \mathrm{g}$ total wereused. B) Analysis of newly synthesized mitochondrial proteins after $\left[{ }^{35} \mathrm{~S}\right]$ methionine labelling. $15 \mu \mathrm{g}$ total were used.

Interestingly, upon loss of TIM21, MITRAC15 displayedincreased association to the MITRAC complex. This could be observed in the eluates of the MITRAC15 ${ }^{\text {FLAG }}$ isolation in HEK293T cells (Figure 38A, lane 5,6) and after $\left[{ }^{35} \mathrm{~S}\right]$ methionine labelling of newly synthesized proteins (Figure 38B, lane 3 , 4). In the analysis of the non-radiolabeled sample, MITRAC15 isolated more MITRAC subunits (COX1, COX4I-1, MITRAC12) in the TIM21 knockdown 
sample, while its interaction with the $\mathrm{P}_{\mathrm{P}}$-module assembly intermediate subunits ND2 and ACAD9 was not changed (Figure 38A). Additionally, in the case of newly synthesized proteins, MITRAC15 specifically isolated more COX1 after TIM21 depletion, while the other interactions were not affected (Figure 38B). The observed accumulation of MITRAC15 seems to be specific for the MITRAC complex. Hence, TIM21 may function as a shuttle to disslocate MITRAC15 from the MITRAC complex.

\subsubsection{ACAD9 is needed for further assembly of the $\mathrm{P}_{\mathrm{P}}$-module}

ACAD9 is one of the first interaction partners of MITRAC15 in the $P_{P}-b$ module assembly and was shown to be required for complex I assembly (Nouws et al., 2010;Guerrero-Castillo et al., 2017).Hence, it can be proposed that a defect in the assembly of complex I would shift the interaction of MITRAC15 to the MITRAC complex. Toanalyze the interactions of MITRAC15 in cells with impaired complex I assembly, an siRNA mediated knockdown ofACAD9 in HEK293T cells expressing MITRAC15 $5^{\text {LAG }}$ was performed and MITRAC15 $5^{\mathrm{FLAG}}$ was isolated by immunoisolation. (Figure 39). 
A
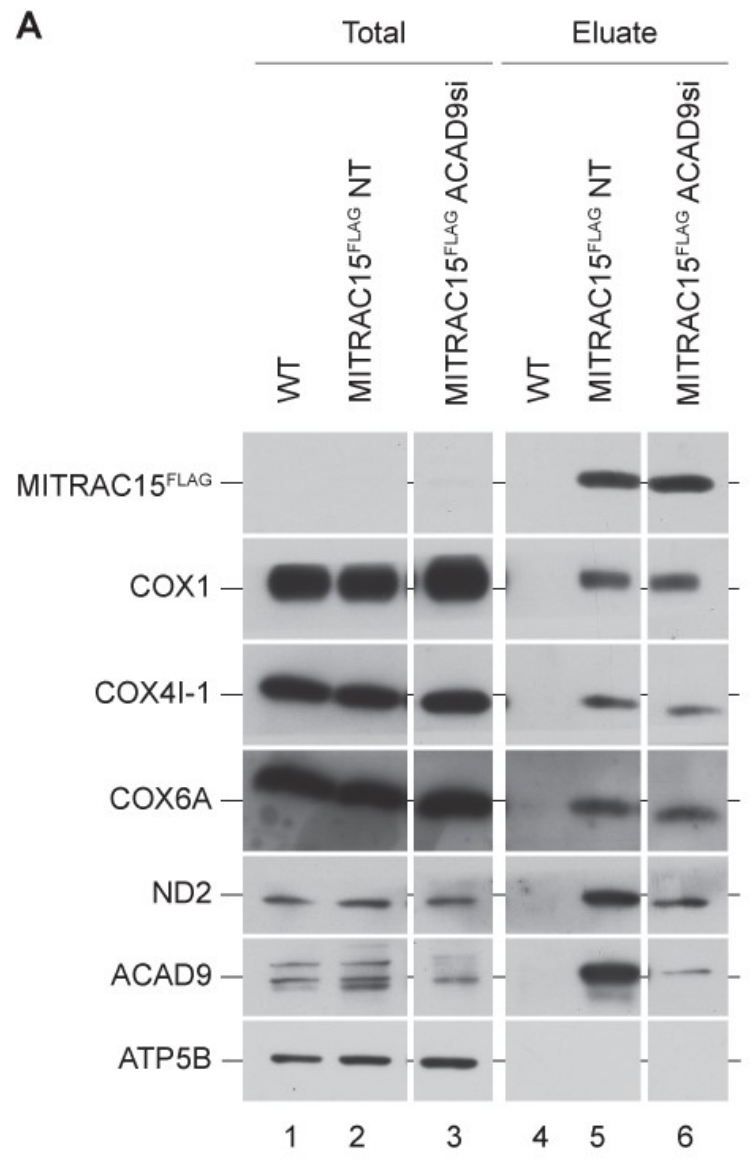

B
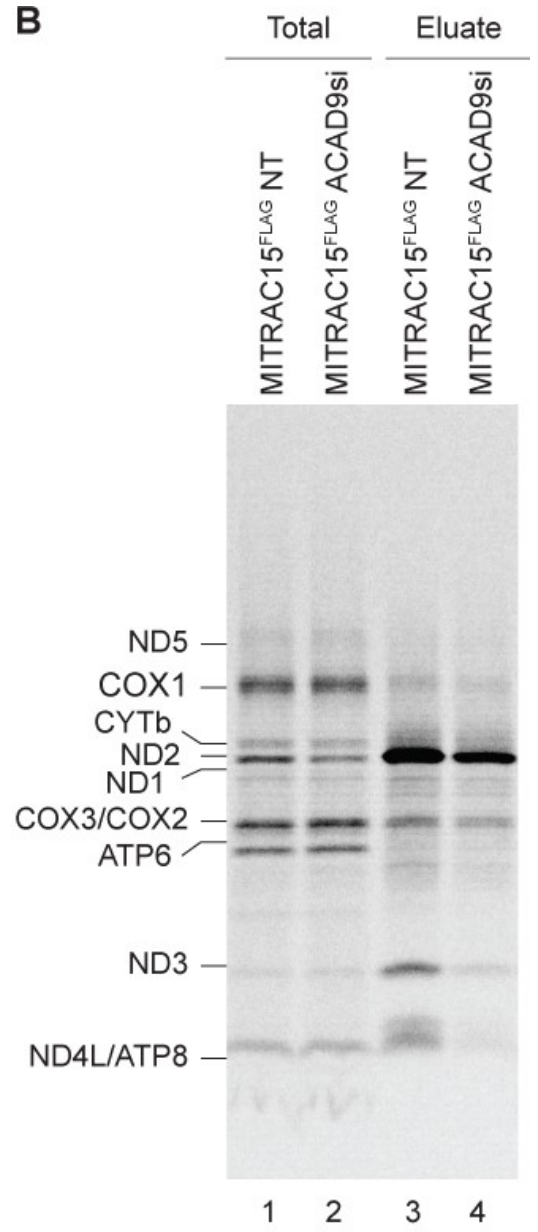

C

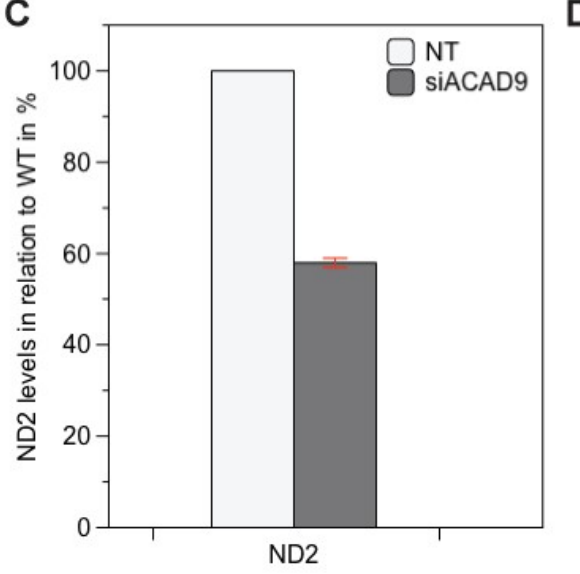

D

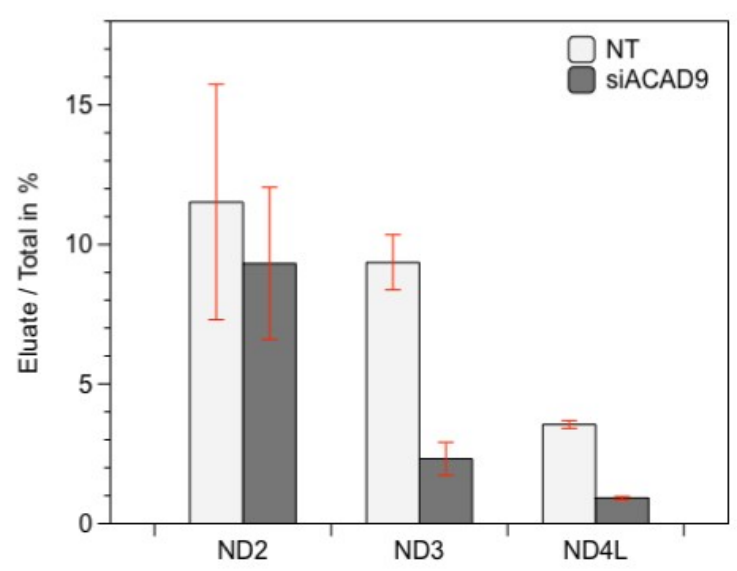

Figure 39ACAD9 depletion impairs assembly of the $P_{\mathrm{P}}$-module, but does not affect ND2 binding of MITRAC15. MITRAC15 ${ }^{F L A G}$ expressing HEK293Tcells were solubilized in buffer containing $1 \%$ digitonin. Immunoprecipitation was performed with FLAG agarose beads. A) Western blot analysis of complex I proteins (ND2, ACAD9) and complex IV proteins (COX1, COX4I-1, COX6A) by antibody probing. $1.4 \%$ total and $100 \%$ of elution. B) Analysis of newly synthesized mitochondrial proteins after ${ }^{35} \mathrm{~S}$ methionine labelling. $1.7 \%$ total and $100 \%$ of elution.C) Relative amount of newly synthesized ND2 standardized to ND2 levels in NT cells.SEM, $N=3$. Protein level in NT set to $100 \%$.D) Amount of $P_{P}$-module subunits in the eluate of $B$ ) in comparison to the total. Protein level in total set to $100 \%$.SEM, $n=3$. 
Unlike the knockdown of TIM21, the depletion of ACAD9 in cells did not cause the accumulation of MITRAC15 in the MITRAC complex. Even though MITRAC15 loses it's interaction with ND2, suggesting a lower association of MITRAC15 with the $P_{P}$ module assembly intermediate, it's interaction with complex IV proteins was not affected (Figure 39A). Hence, the function of MITRAC15 in complex I and IV seems to be independent from each other. Also,knockdown of ACAD9 did not lead to a reduction of total ND2 protein levelsin the cell (Figure 39A, lane 2,3), but only reduced the interaction of MITRAC15 with ND2 (Figure 39A, lane 5, 6). Therefore,ACAD9 might be needed for the interaction of MITRAC15 with ND2. However,this assumption is contradicted by the interaction of MITRAC15 with newly synthesized mitochondrial proteins(Figure 39B - D). Even though the effectiveamount of newly synthesized ND2 that coisolated with MITRAC1 $5^{\mathrm{FLAG}}$ was lower in the siACAD9 treated sample compared to the NT sample (Figure 39B, lane 3,4), the relative amount of newly synthesized ND2 was similar in both samples (Figure 39D). The lower effective association of MITRAC15 with newly synthesized ND2 in the siACAD9 treated sample compared to the NT sample is caused by a reduction in the synthesis of ND2 by $40 \%$ (Figure 39C).

In consideration, a more fitting explanation for the reduction of ND2 association with MITRAC15 is that the reduction of ACAD9 protein levels in cells impairs the further assembly of the $\mathrm{P}_{\mathrm{P}}-\mathrm{b}$ module. The impaired assembly causes the ND2 and MITRAC15 containing complex to disassemble. However, siRNA mediated depletion of ACAD9 also caused a reduction in the interaction of newly synthesized ND3 and ND4L with MITRAC15 ${ }^{\text {FLAG }}$ (Figure 39B, lane 3,4). This observation could also be confirmed in the quantification of the relative amount of coisolated ND3 and ND4L, which wasnormalized to the protein levels in the totals (Figure 39D). This might be explained by the fact that ND3 and ND4L join the $\mathrm{P}_{\mathrm{P}}-\mathrm{b}$ module at a later stage (GuerreroCastillo et al., 2017), which implies that ACAD9 is required for $\mathrm{P}_{\mathrm{P}}-\mathrm{b}$ module maturation at a later stage than MITRAC15. Therefore, the obstruction of the $\mathrm{P}_{\mathrm{P}-\mathrm{b}}$ module assembly, caused by depletion of ACAD9, may also leads to the disassembly of the MITRAC15 and ND2 containing complexpreventing the binding of ND3 and ND4L. 


\subsubsection{Generation and analysis of a MITRAC $15^{-/}$cell line}

To characterize the function of MITRAC15 in more detail, a knockout cell line (MITRAC15 ${ }^{-/}$) was generated using CRISPR/CAS9-mediated genome editing. The MITRAC15 gene consists of 5 exons, where the first exon only encodes the first 5 amino acids. Therefore, for the generation of a MITRAC15 $5^{-/}$cell line, both the second and the third exons were targeted. After single cell sorting and cell expansion, colonies were analyzed byWestern blotting. Clones where the signal for MITRAC15 was absent were further analyzed. To verify the knockout, PCR products of the MITRCA15 gene were sequenced. One clone had a homozygous stop codon insertion in exon 2. This mutant was chosen for further characterization (Figure 40). The other clones carried point mutations or frame shift mutations within the MITRAC15 gene.

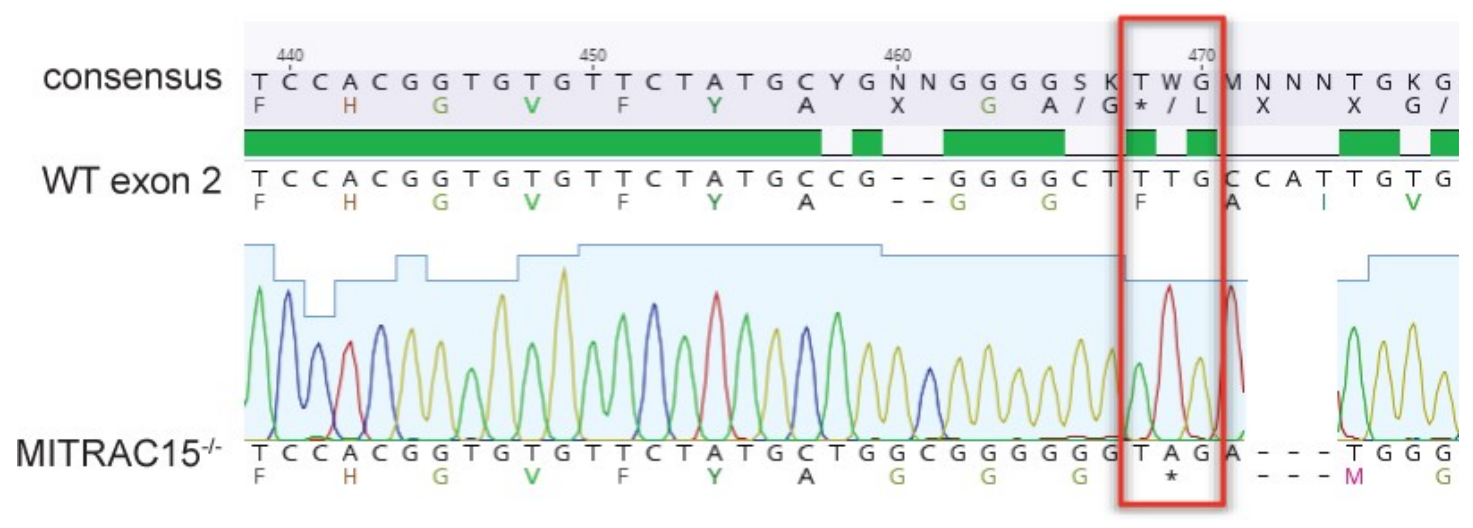

Figure 40 Nucleotide alignment of sequenced MITRAC15 ${ }^{-1-}$ clone and WT exon 2 sequence. Position of the stop codon is highlighted.

\subsubsection{MITRAC15 affects ND2 synthesis but not complex I and complex IV activity}

Since MITRAC15 is involved in the biogenesis of complex I and complex VI, It was assumed that a knockout of MITRAC15 would affect the stability or synthesis of mitochondrial-encoded subunits in complex I and IV. In particular, ND2 might be influenced by the knockout of MITRAC15, since MITRAC15 showed strong interaction with newly synthesized ND2 (Figure 36) and potential nascent chains of ND2 (Figure 37). Therefore, the effect of 
MITRAC15 knockout was investigated on complex IV and complex I subunits by different biochemical approaches (Figure 41).

A
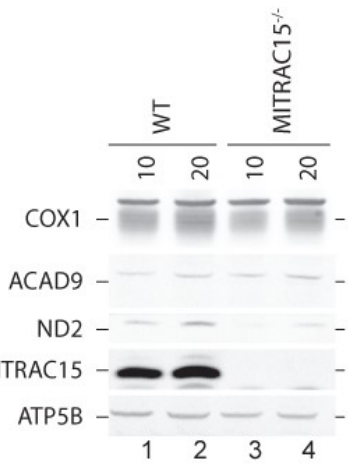

D

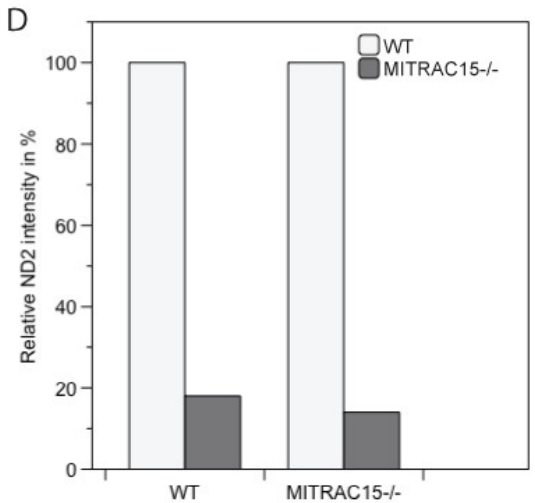

B

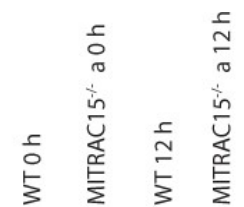

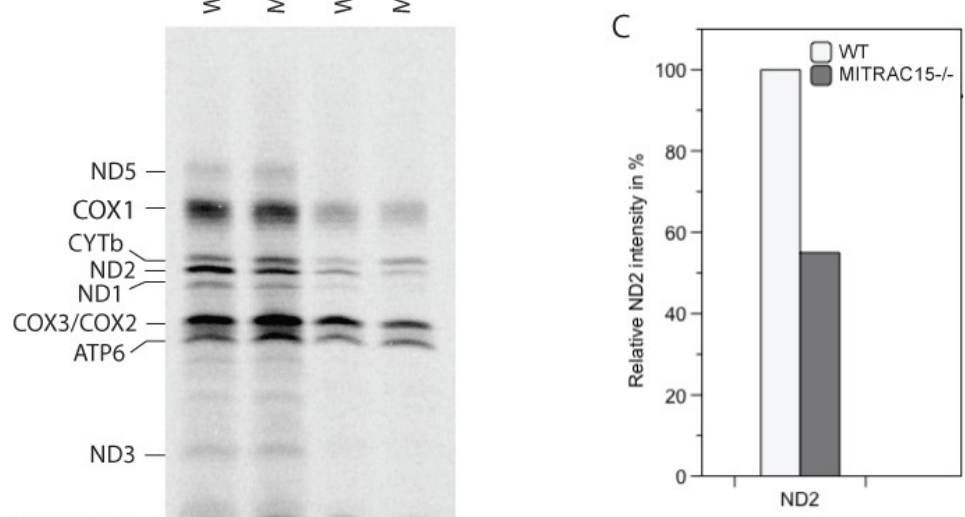

Figure 41 ND2 steady state levels and synthesis is decreased in MITRAC1 $5^{-/-}$cells. A) WT and MITRAC1 $5^{-1}$ HEK293Tmitochondria were solubilizedin buffer containing $1 \%$ digitonin. B) $\left[{ }^{35} \mathrm{~S}\right]$ methionine labelling of newly synthesized mitochondrial proteins were performed in WT and MITRAC1 $5^{-/}$HEK293Tcells with $1 \mathrm{~h}$ pulse and harvested directly or after $12 \mathrm{~h}$ chase in $\left.{ }^{35} \mathrm{~S}\right]$ methionine free medium. C) Quantification of ND2 signal intensityof pulse labelled samples in B) relative to the WT sample. Values normalized to ATP8 protein levels. $n$ = $1 \mathrm{D}$ ) Quantification of ND2 signal intensity of samples in B) relative to the pulse samples. Values normalized on ATP8 protein levels. $n=1$

Firstly, steady state protein levels from isolated mitochondria were analyzed (Figure 41A).A specific reduction in ND2 was observed as a result of knockout of MITRAC15. COX1, the core complex IV subunit and ACAD9, another assembly factor of the $P$ module, were not affected.Furthermore, newly mitochondrial-synthesized proteins were labelled with $\left[{ }^{35} S\right]$ methionine to investigate changes in mitochondrial protein synthesis (Figure 41B, lane 1, 2). Since a reduction in ND2 synthesis was already visible after the pulse 
labelling (Figure 41B, lane 1, 2), the relative ND2 signal intensity, ofthe pulse labelled samples was quantified for a more precise comparison (Figure 41C). The signal intensities were normalized to the intensity of the ATP8 signal. Furthermore, the normalized ND2 signal intensity of the WT sample was set to $100 \%$, while the normalized ND2signal intensity in the MITRAC $15^{-/}$sample was calculated relative to the WT sample. Quantification revealed a reduction of ND2 synthesis by $44 \%$ in MITRAC $15^{-1-}$ cells (Figure $41 \mathrm{C}$ ). To assess the stability of the radiolabeled mitochondrial-synthesized proteins, a second set of $\left[{ }^{35} \mathrm{~S}\right]$ methionine labelled cells were chased for $12 \mathrm{~h}$ with radioactive-free medium before harvesting (Figure $41 \mathrm{~B}$, lane 3,4 ). To assess the stability of ND2, the signals in all 4 samples (Figure 41B) were quantified and normalized to ATP8. The normalized ND2 signal in the pulse labelled samples were set to $100 \%$ and the normalized ND2 signal in the chased samples were calculated relative to the corresponding pulse labelled sample (Figure 41D). The quantification of the $12 \mathrm{~h}$ chase showed that the relative decrease of the radiolabeled ND2 signals were similar in WT (82\% reduction) and MITRAC15 ${ }^{-1-}$ (86.5\% reduction) cells, showing that a knockdown of MITRAC15 does not affect the turnover rate of ND2.Therefore, MITRAC15 has a role in ND2 synthesis, but no influence onit's stability.

ND2 is one of the structural core subunits of mitochondrial complex I and actively takes part in proton transfer. Hence, the reduction of ND2 protein levels in MITRAC $15^{- \text {- }}$ cells might also influence the net activity and stability of complex I. Complex I oligomerises with complex III and IV into supercomplexes to enhance the electron transfer between the OXHPOS complexes (Bianchi et al., 2004). Therefore, a defect in complex I might also affect the performance of complex III and IV. To investigate the validity of this assumption, an ELISA assay was performed to quantify complex I and IV activity in mitochondria (single complexes and different supercomplexes) (Figure 42).Mitochondria were solubilized with the buffer provided along with the kit and complexes, I or IV, were immobilized in wells of a 96 well plate with coupled antibodies. The measurements were performed in the wells. 


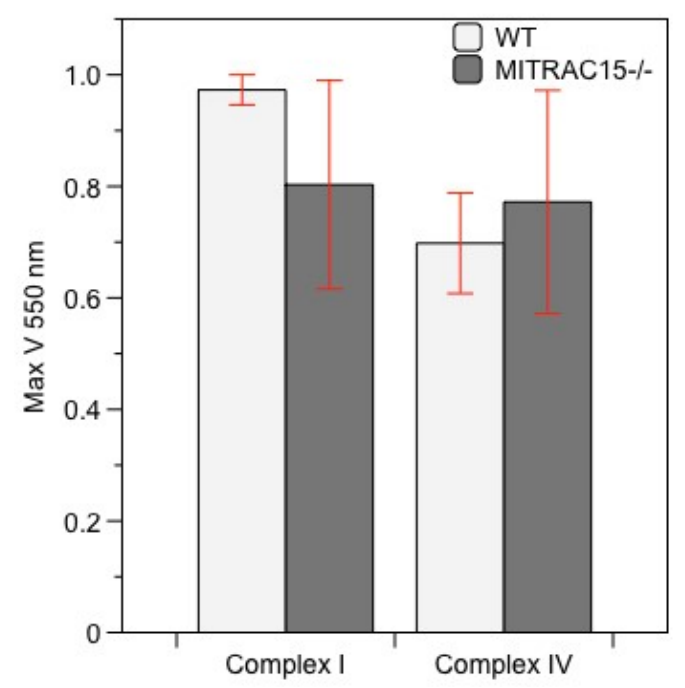

Figure 42 MITRAC15 ${ }^{-1-}$ cells display similar complex IV and complex I activities to WT cells. ELISA assay kits were used with WT and MITRAC1 $15^{-1}$ HEK293Tcells. SEM, $n=3$.

Despite the severe reduction of ND2 in MITRAC15 ${ }^{-/}$cells on steady state, the activities of complex IV and complex I were not significantly affected. This finding contradicts the reduced production of nascent ND2 as observed in Figure 41 . This can either be explained by the fact that the remaining ND2 serves to form assembled complex I similar to WT cells, or complex I retains its stability and catalytic activity even without ND2 being present.

\subsubsection{MITRAC15 ${ }^{--}$causes increase an in complex IV assembly}

Despite reducing ND2 synthesis by $45 \%$ (Figure 41), a knockout of MITRAC15 did not cause a significant reduction in the activity of the OXPHOS complexes I and IV (Figure 42). To further investigate the consequences of the MITRAC15 knockout, a more sensitive method was chosen to monitor the assembly of complex I and IV. Protein imports of $\left.{ }^{35} \mathrm{~S}\right]$ methionine-labelled subunits of complex I and complex IV into mitochondria were performed and their assembly into the mature complexes was monitoredby BN-PAGE. Since assembly defects are most obvious by the investigation of late complex constituents, the NDUFB8 and COX6A subunits were chosen for complex I and IV respectively (Figure 43). Both NDUFB8 and COX6A assemble at late stages into their corresponding complexes (Lazarou et al., 2009), (GuerreroCastillo et al., 2017). 


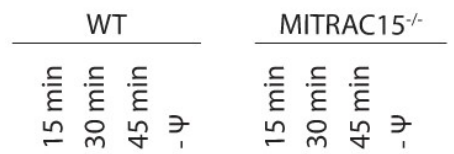

$\mathrm{kDa}$

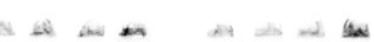

$250-$
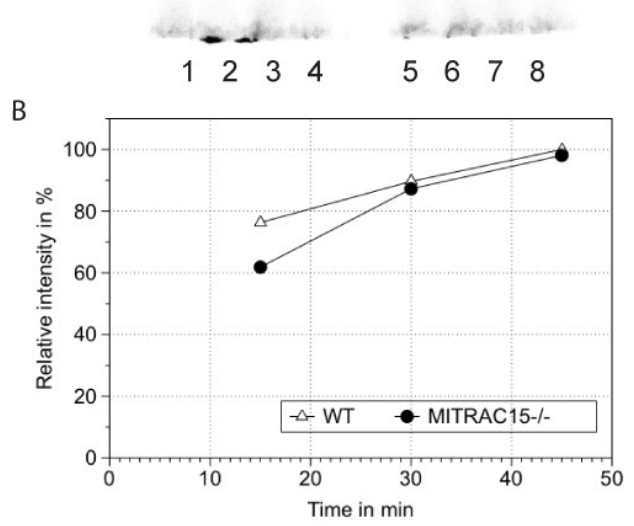

Import of COX6A

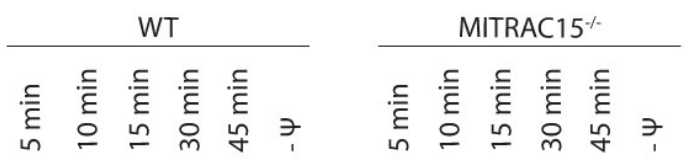

$\mathrm{kDa}$

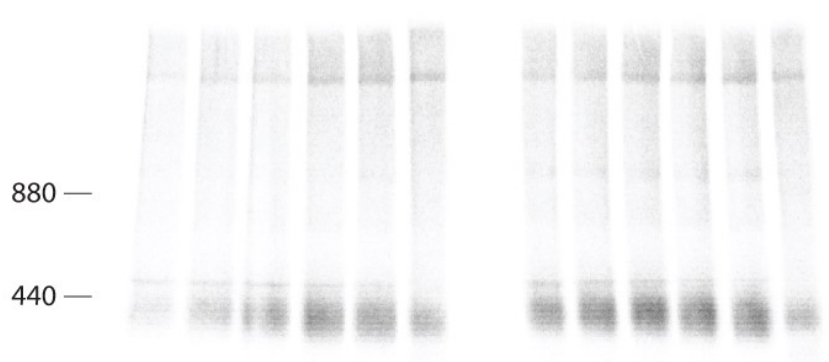

$250-$

$70-$
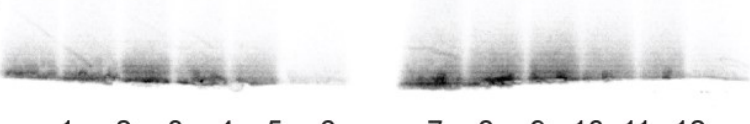

$\begin{array}{llllll}7 & 8 & 9 & 10 & 11 & 12\end{array}$

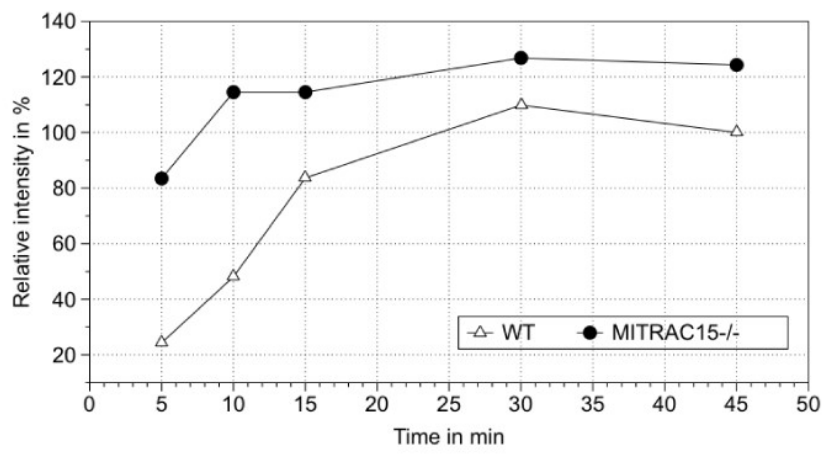

Figure 43 MITRAC $15^{-1}$ has no effect in the assembly of complex I but leads to increase in complex IV assembly.Radiolabeled NDUFB8 and COX6A were imported into HEK293TWT and HEK293T-MITRAC15 ${ }^{-/}$mitochondria. A) Mitochondria were solubilized in buffer containing $1 \%$ digitonin after import, $90 \mu \mathrm{g}$ solubilized mitochondria were subjected to $\mathrm{BN}$ PAGE (NDUFB8: $2.5-10 \%$; COX6A: $4-13 \%$ ) to observe assembly of the subunits. B) Quantification of the assembly of NDUFB8 and COX6A into their complexes in A). Values are relative intensities normalized on the intensity of the WT sample at the last time point.

The import and assembly of NDUFB8 occurred into a complex of around 440 $\mathrm{kDa}$, corresponding to the predicted size of the $\mathrm{P}_{\mathrm{D}}-\mathrm{b}$ module (GuerreroCastillo et al., 2017). The further assembly of this intermediate into the mature complex I, or later intermediate assembly stages,could not be seen in this experiment. However, knockout of MITRAC15 did not affect the assembly of NDUFB8 into the $P_{D}-b$ module.

Interestingly, a clear increase of assembled COX6A into complex IVcould be observed. However, the import efficiency of COX6A was similar between MITRAC15 ${ }^{-/-}$and WT cells. Hence, it is tempting to speculate that MITRAC15 
is involved in quality control during complex IV assembly steps.Furthermore, it has to be mentioned that even though the import of COX6A was largely membrane potential dependent, a fraction of the protein was still imported and assembled into the mature complex IV while membrane potential was absent (Figure 43 COX6A, lane 6, 12).Similar import behaviour has been reported for other small proteins located in the inner mitochondrial membrane (Wagner et al., 2009). 


\section{Discussion}

\subsection{Assessing the composition of the mitochondrial ribosome-associated MITRAC}

The regulation of mitochondrial translation has been characterized in detail forthe yeast Saccharomyces cerevisiae. A number of translation factors are known to act on either the mitochondrial mRNA, or to interact with the mitochondrial ribosome to promote the translation(Dennerlein et al., 2017). In humans, the regulation of mitochondrial translation is mostly enigmatic. Aside from TACO1, which is known to interact with the COX1 mRNA(Richman et al., 2016), only C12ORF62 and MITRAC 12 was shown to specifically affect the translation of an individual mitochondrial protein, COX1(Mick et al., 2012). Originally characterized for its role in COX1 translation (Weraarpachai et al., 2012),(Mick et al., 2012), Richter-Dennerlein et al. were able to show that C12ORF62 is present during the translation of COX1 and can copurify its nascent chains when the mitochondrial translation is stalled(RichterDennerlein et al., 2016). In this study, early stage MITRAC, which is associated with the mitochondrial ribosome $\left(\right.$ MITRAC $^{\text {ribo }}$ ), was isolated. This was used to generate a structural model using cryoEM.

\subsubsection{Early assembly of complex IV}

In the Western blot analyses of the purified MITRAC ${ }^{\text {ribo }}$, we were able to see not only mitochondrial ribosome subunits, but also the structural subunit COX4I-1 and the shuttle protein TIM21 (Figure 24, Figure 28). This indicates that the assembly of the cytochrome $c$ oxidase already begins during or shortly after COX1 translation, at which point the ribosome-association of MITRAC still prevails. Our findings contradict with the early assembly models proposed recently. Bourens et al. (Bourens and Barrientos, 2017a)characterized $\mathrm{CMC1}$ as an assembly factor of complex IV, which is present in MITRAC after it has dissociated from the mitochondrial 
ribosome(MITRAC ${ }^{\text {free }}$ ). CMC1 was shown to interact with MITRAC12, C12ORF62 and COX1 and it also comigrates with them in the MITRAC free complex. An interaction of CMC1 and COX4I-1 was not detected. Therefore, Bourens et al. proposed that $\mathrm{CMC} 1$ joins MITRAC free and disassembles from the complex prior to the assembly of COX4I-1. MITRAC ribo, which is associated with the COX1 translating mitochondrial ribosome, predates the formation of MITRAC ${ }^{\text {free }}$. The observation that COX4I-1 already associates with MITRAC ${ }^{\text {ribo }}$ (Figure 24, Figure 28) contradicts the claim by Bourens at al. that COX4I-1 assembles onto MITRAC free after the disassembly of CMC1.Vidoni et al. (Vidoni et al., 2017) proposed a similar assembly model of complex IV in a study about the newly identified assembly factor MR-1S. In a mass spectrometry of mitochondria solubilized with DDM and loaded onto a blue native gel, Vidoni et al. identified a small complex containing HIGD1A, COX4I-1 and COX5A. They propose that this complex represents a modular assembly intermediate, which assembles onto MITRAC ${ }^{\text {free }}$. Because of the use of DDM as a detergent, these results are arguable. Unlike digitonin, DDM is a rather harsh detergent, which disturbs the interaction of COX4I-1 with MITRAC (Figure 20). Therefore, it is questionable whether the HIGD1A, COX4I-1 and COX5A complex forms on its own in vivo, or disassembles from a complex IV assembly step. Nevertheless, it is necessary to verify the presence of COX5A with MITRAC ${ }^{\text {ribo }}$ to further clarify this observation. Taking the results ofVidoni et al. (Vidoni et al., 2017) and Bourens et al. (Bourens and Barrientos, 2017a) into account, it is possible that variations exist in the early assembly of complex IV, allowing cytosolic translated subunits to be added to MITRAC at different steps. Figure 44shows a putative early assembly model for the mitochondrial complex IV. 


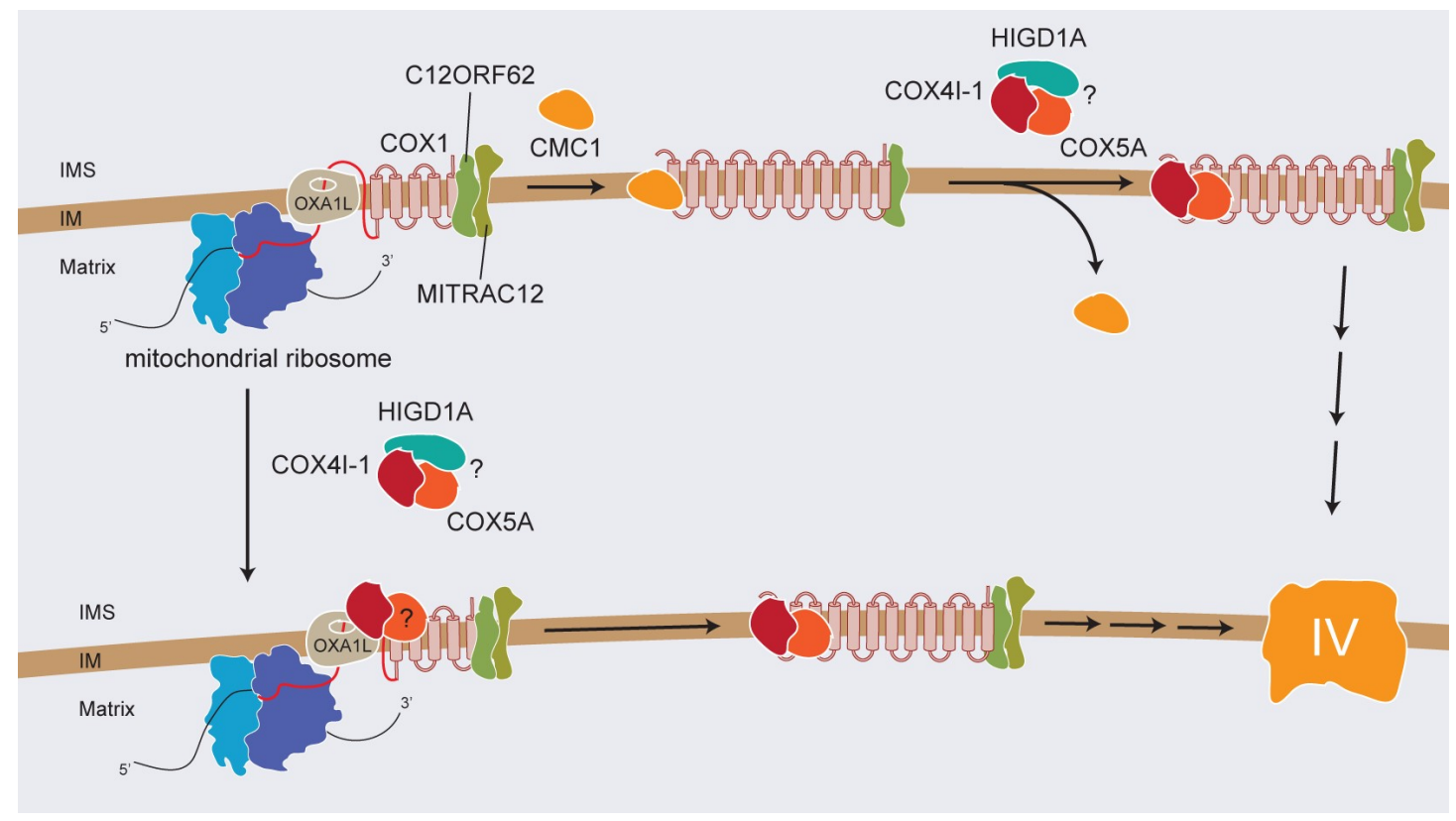

Figure 44 Putative early assembly of mitochondrial complex IV. Assembly of nuclear encoded subunits (COX4I-1, COX5A?) into MITRAC can occur during COX1 synthesis or after MITRAC disassociates from the mitochondrial ribosome. CMC1 participate in complex IV assembly if COX4I-1 is not assembled into MITRAC during COX1 translation.

\subsubsection{Structure of MITRAC ribo}

In recent years, several milestones were set in the study of mitochondrial ribosomes by the use of electron microscopy. CryoEM analyses yielded high resolution structural data for mitochondrial ribosomes in human (Amunts et al., 2015), yeast (Desai et al., 2017) and porcine (Greber et al., 2015b) and provided an insight into the mechanism of this complex machinery. Nevertheless, the process of translation initiation in human mitochondrial ribosomes has remained enigmatic. The MITRAC subunit,C12ORF62, is one of the few known human translation factors and was shown recently to associate with the COX1 translating ribosome (Richter-Dennerlein et al., 2016). By using cryoEM, we hoped to gain more insight into the interaction between MITRAC and the mitochondrial ribosome.

The traditional detergents used to isolate the mitochondrial ribosome and the cytochromec oxidase are Triton X-100 and digitonin respectively. While Triton X-100 provides high solubilisation capacities for mitochondrial proteins (Spremulli, 2007), digitonin provides mild solubilisation conditions for complex IV assembly intermediates (Mick et al., 2012). Still, both detergents were not 
suitable for our purpose, because Triton X-100 disturbs the interaction between MITRAC and the mitochondrial ribosome (Figure 20) and the solubilisation capacity of digitonin is insufficient (Figure 21). After assessing the stringency (Figure 20) and solubilisation capacity (Figure 21) of several detergents, PCC turned out to be the optimal detergent for this purpose. It belongs to a group of recently developed detergents for crystallography (Hovers et al., 2011) and provided both mild solubilisation conditions, to keep the complex intact and high solubilisation capacity to provide a concentrated sample for cryoEM analysis.

Even though a sample for cryoEM analysis could be generated, the sample quality was rather poor. A high amount $(72 \%)$ of the 1,132,000 picked particles had to be discarded as unusable. Furthermore, a large part of the isolated complexes are not the mitochondrial monosome, but consist of only the large subunit. Since the mitochondrial ribosomes isolated with MITRAC should be stalled in the process of COX1 translation, they should be present as monosomes and not single large subunits. The high amount of free large subunits and not usable particles indicates a decay of the isolated complexes during or after the isolation. Hence, optimizing buffer and isolation conditions might raise the yield of suitable complexes.

Nevertheless, the average resolution of the isolated large subunits could be refined to $4.7 \AA$, which is still a lowerresolution than the $3.4 \AA$ of the existing structure of the large mitochondrial ribosomal subunit (Brown et al., 2014), but sufficient to see structural details of proteins and the 16S rRNA. Still, this resolution is only present in the structure of the ribosomal subunit itself. The putative MITRAC complex can only be visualized at a lower resolution that does not provides information of its composition. The cause of the low resolution is most likely the high mobility of the complex, which is known for causing lower resolutions for cryoEM analysis in general (Subramaniam et al., 2016). The mobility of the putative MITRAC complex on the ribosome could be caused by the intrinsic flexibility of the ribosome-associated MITRAC, which can be influenced by the conformation changes during COX1 translation. An argument for the influence of the mitochondrial translation process on MITRAC flexibility is the subset of structures generated by a second classification step from the pool of 133,000 particles of the large 
ribosomal subunit (Figure 33). Among these three structures, the putative MITRAC complex shows the highest flexibility while the putative t-RNA density is not present in the active site. In contrast it's flexibility is the lowest when the putative "L" shaped tRNA density is oriented parallel to the central protuberance-exit tunnel axis. If the status of the putative t-RNA density represents different steps during translation, this suggests that changes in the flexibility of MITRAC dependent on the translation procedure.

The low-density structure of the putative MITRAC can provide an insight into the localization of the complex relative to the mitochondrial ribosome. Both the structure of the free large subunit and the monosome show that the putative MITRAC density interacts with the large subunit of the mitochondrial ribosome, while it is localized at the exit tunnel. Considering C12ORF62 can isolate nascent COX1 chains released from the mitochondrial ribosome by puromycin(Richter-Dennerlein et al., 2016), C12ORF62 should interact with the nascent COX1 chains directly. This is facilitated by the localization of MITRAC at the exit tunnel of the mitochondrial ribosome. Furthermore, the insertase OXA1L (Bonnefoy et al., 1994) could be coisolated with MITRAC ${ }^{\text {ribo, }}$, which suggests a role of OXA1L in the insertion of the nascent COX1 into the inner mitochondrial membrane, as has been shown in yeast (Bonnefoy et al., 2009). Even though OXA1L was shown to substitute its homologue Oxa1 in yeast (Bonnefoy et al., 1994), a shRNA knockdown of OXA1L only caused a decrease in steady state levels of complex I and V, whereascomplex III and IV were not affected by a knockdown of OXA1L (Stiburek et al., 2007). These data imply the possibility, that other uncharacterized proteins in human mitochondria can substitute the function of OXA1L.

To more thoroughly assess the composition and interaction of the MITRAC subunits relative to each other and also with the mitochondrial ribosome, more structural data is required. A way to improve structural data in general is to reduce the flexibility of the target. Chloramphenicol is an antibiotic that stalls translation in bacteria bybinding to the A site of the active center of the bacterial ribosome, thereby enhancing the stability of the monosome (Pathak et al., 2016;User, 2001).Specifically, chloramphenicol binds to the 23S rRNA of the $50 \mathrm{~S}$ large subunit in bacteria and interacts with 7 bases localized between the position 2061 and 2506 (User, 2001).The A site is 
largelyconserved between the human mitochondrial ribosome and its bacterial ancestors except for the missing A finger (position 827-942) and UL25m (Brown et al., 2014), both of which do not participate in the interaction with chloramphenicol. Hence, chloramphenicol might also be used to stall mitochondrial translation at a specific step. Nevertheless, the intrinsic flexibility of the ribosome-associated MITRAC still remains. A more promising way to improve the resolution of the present structural model is to perform

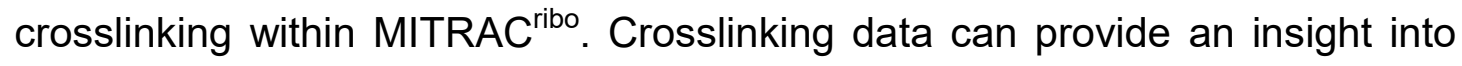
the interaction sites of MITRAC subunits, both with each other and with the mitochondrial ribosome, which together with the obtained structure, would improve the understanding of the structure and composition of MITRAC ribo.

\subsection{MITRAC15, acoordinator of complex I and IV assembly}

Initially characterized in a study by Mick et al. in 2012 (Mick et al., 2012), MITRAC15 was shown to associate with subunits of complex I and IV and was implicated in the assembly of both complexes. More recently, Guerrero Castillo et al. (Guerrero-Castillo et al., 2017) undertook a systematic approach to define assembly modules for complex I and showed that MITRAC15 comigrates in the earliest assessable assembly intermediate of the $P_{P}-b$ module. In this thesis, further insights into MITRAC15 function could be provided. The key findings that come from the work presented in this thesis are: 1) MITRAC15 is present in distinct assembly intermediates of complex I and IV 2) A knockdown of TIM21 increases the association of MITRAC15 with the MITRAC complex. 3) A MITRAC15 ${ }^{-/-}$cell line displays enhanced assembly of complex IV and decreased synthesis of ND2. 4) ACAD9 could be shown to be crucial in promoting the assembly of later subunits of complex I, like ND3 or ND4L, into the $\mathrm{P}_{\mathrm{P}}-\mathrm{b}$ module. 5) MITRAC15 shows strong association with newly synthesized ND2 and can coisolate nascent chains of mitochondrialsynthesized proteins after puromycin treatment.

By performing 2D PAGE analysis of isolated mitochondria, several complexes containing MITRAC15 were detected(Figure 35). It was observed that MITRAC15 associates with ACAD9 in a complex with an apparent migration 
around $440 \mathrm{kDa}$ on BN-PAGE, which would fit the early $\mathrm{P}_{\mathrm{P}}-\mathrm{b}$ module intermediate, which has a size around $400 \mathrm{kDa}$ (Guerrero-Castillo et al., 2017). MITRAC15 also associates with MITRAC12 in the MITRAC complex (Mick et al., 2012). Interestingly, MITRAC15 is present in an additional uncharacterized complex around $70 \mathrm{kDa}$, which neither contains MITRAC12, nor ACAD9. Considering that MITRAC15 fulfils functions in complex I and IV assembly, this low molecular weight complex might represent a resting pool of MITRAC15, which can be utilized either in complex I or in complex IV assembly, with other uncharacterized subunits.

Interestingly the presence of TIM21 restricted the interaction of MITRAC15, specifically with complex IV. When TIM21 was knocked down, an increase in MITRAC15 interaction with the MITRAC subunits was observed. However, there was no change in the interaction of MITRAC15 with complex I (Figure 38). This observation also argues in favour of a free pool of MITRAC15, because the increased association of MITRAC15 with complex IV did not diminish its interaction with complex I. A TIM21 knockdown can contribute to the accumulation of MITRAC15 in the MITRAC complex in two possible ways: a) TIM21 acts as a shuttle to transfer imported proteins to the MITRAC complex for further assembly (Mick et al., 2012). Therefore, a knockdown of TIM21 would lead to slower complex IV formation, thus accumulating MITRAC15 in the MITRAC complex (Figure 45A). b) TIM21 performs general shuttle functions and actively transports MITRAC15 from the MITRAC complex to the free pool of MITRAC15. This is supported by the observation of Mick et al. (Mick et al., 2012) that the interaction between TIM21 and MITRAC15 remains the same regardless of whether TIM21 is shifted to the translocase or to OXPHOS assembly intermediates (Figure 45B). 


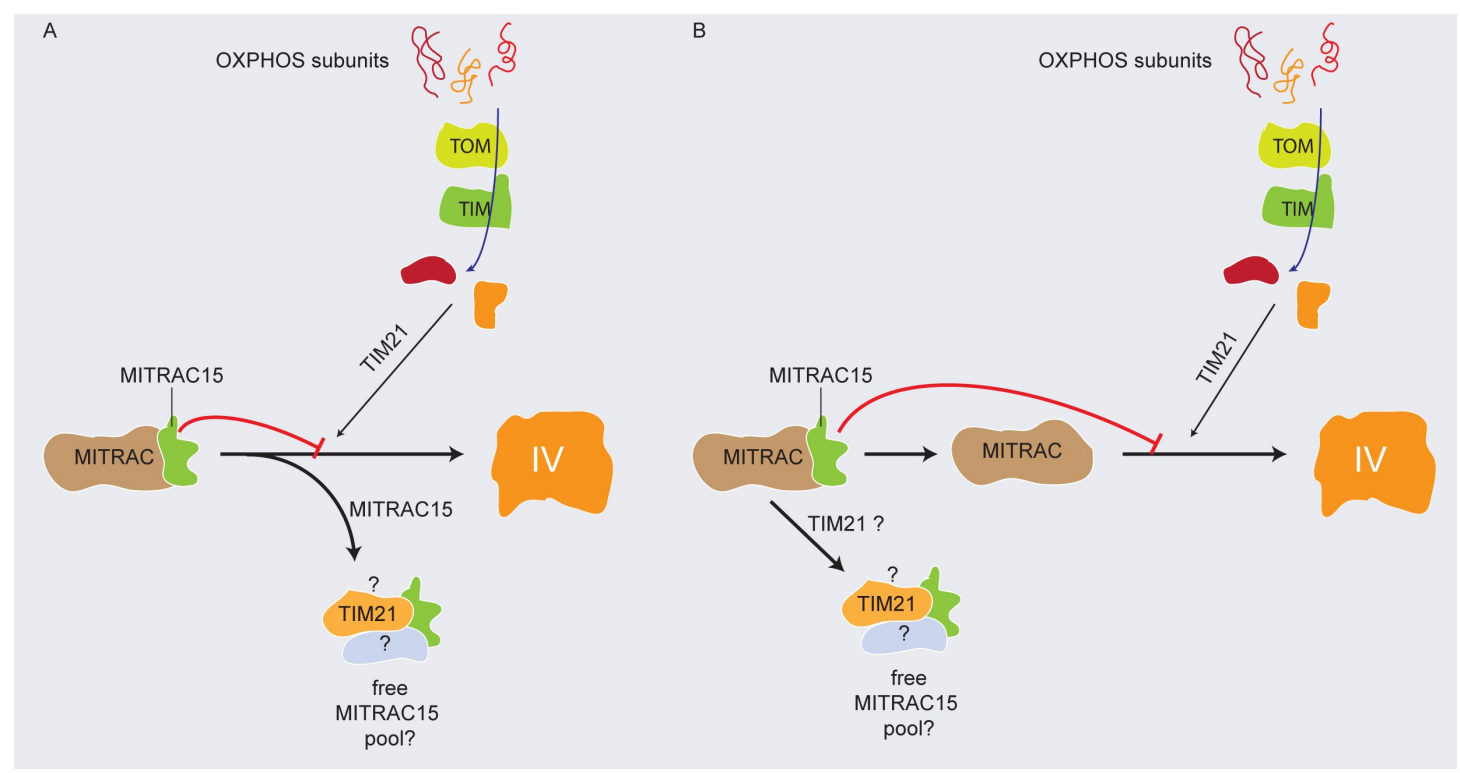

Figure 45 TIM21 promotes the disassembly of MITRAC15 from MITRAC. A) MITRAC15 disassembles from MITRAC during further complex IV assembly and TIM21 indirectly causes the disassembly of MITRAC15 by promoting complex IV assembly. B) TIM21 directly shuttles MITRAC15 away from MITRAC to an ambient pool.

A protein which is present in the early assembly intermediate of the $P_{\mathrm{P}}-\mathrm{b}$ module with MITRAC15 is ACAD9, making it one of the first interaction partners of MITRAC15 that is required for complex I assembly. The knockdown of ACAD9 impaired the assembly of the $P_{\mathrm{P}}-\mathrm{b}$ module and caused a lower association of MITRAC15 with ND2. The interaction of MITRAC15 with the MITRAC complex was not affectedunder these conditions (Figure 39). This result implies that MITRAC15 carries out its functions in complex I and IV independently and does not function in the crosstalk of both assemblies. It also suggests that MITRAC15 cannot be shifted directly between complex I and IV and hasto be returned to a free pool of MTIRAC15. The free pool of MITRAC15 is possibly represented by the low molecular weight MITRAC15containing complex at $70 \mathrm{kDa}$ on BN-PAGE. To clarify the function of this complex, MITRAC15 containing complexes could be isolated and separated by BN PAGE. A mass spectrometry analysis would then provide an insight into the composition of the complex and functional analysis of these components could be carried out. Figure 46 shows a model of MITRAC15 migration between its different complexes. 


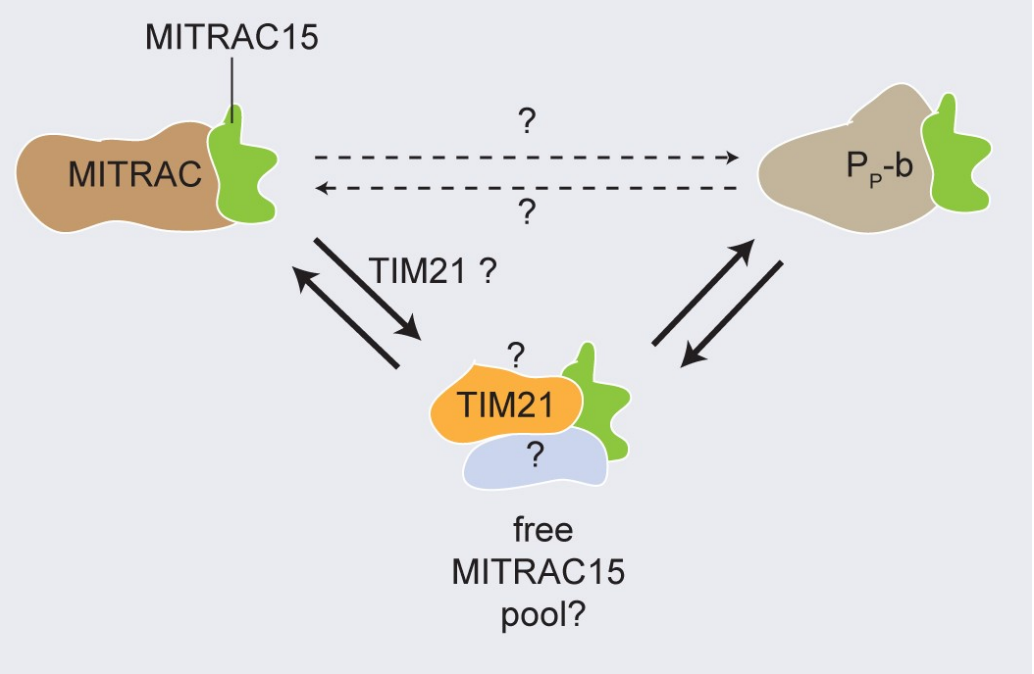

Figure 46Migration of MITRAC15 between complexes. MITRAC15 is likely to be transferred to an uncharacterized complex first after its function in complex I or IV assembly before participation in a new assembly event.

Interestingly, a knockout of MITRAC15 did not affect the activity of the cytochrome $c$ oxidase (Figure 42). On the contrary, the assembly of the late subunits of complex IV, such as COX6A, was even increased in MITRAC15 cells. Therefore, MITRAC15 might be involved in quality control of complex IV assembly. Since the activity of complex IV was not affected in MITRAC15 $15^{-1}$ cells, other aspects of complex IV functionality, such as the production of reactive oxygen species (ROS) have to be analysed. A possible reason to stall complex IV assembly is to define the fate of the complexes, whether they will remain as monomeric complex IV, or assemble into one of the super complexes. In this scenario, MITRAC15 might retain complex IV biogenesis until the other complexes can be provided for supercomplex formation. Supercomplex formation is known to enable a higher efficiency of electron transfer (Bianchi et al., 2004) and reduce ROS production (Lopez-Fabuel et al., 2016). These processes could be assessed in MITRAC15-/- cells to investigate the putative effect of complex IV association and disassembly processes. But it has to be considered that measurements of cellular ROS would not be able to discriminate the source of the ROS production. Nevertheless, MITRAC15 is the first characterized protein acting in human complex IV assembly, which is able to stall the process. 
Furthermore, considering its presence in an early $P_{P}-b$ module assembly intermediate the role of MITRAC15 was also assessed in the assembly of mitochondrial complex I.Since in MITRAC15 ${ }^{-/-}$cells ND2 synthesis is decreased by $44 \%$, it can be concluded that MITRAC15 is possibly involved in the synthesis of ND2 (Figure 41). Comparing this effect to a knockout of assembly factors such as C12ORF62, which abolish COX1 synthesis completely (Sven Dennerlein, unpublished data), MITRAC15 might only play an assisting or regulatory role in ND2 synthesis. Therefore, another translation factor may exist for the synthesis of ND2. Possible candidates would be other assembly factors present in the early assembly complex of the $P_{P}-b$ module (ECSIT, NDUFAF1). These have to be investigated with regard to ND2 synthesis. Even though a knockdown of ACAD9 also caused a decrease of ND2 synthesis (Figure 39), it more likely plays an indirect role in ND2 synthesis, considering that ACAD9 does not interact with nascent chains of mitochondrial synthesized proteins(Figure 37). Furthermore, ribosomeassociation of MITRAC15 was shown in an experiment performed by Ricarda Richter-Dennerlein(Richter-Dennerlein et al., 2016; unpublished data, Richarda Richter-Dennerlein). MITRAC15 shows strong interaction with newly synthesized ND2 and can copurify nascent chains of mitochondrialsynthesized proteins (Figure 37). The nascent chains most likely belong to ND2, but have to be further characterized. Therefore, MITRAC15 most likely associates with ND2 during its translation, while ACAD9 joins after dissociation from the mitochondrial ribosome. Despite showing strong interaction with newly synthesized ND2, ACAD9 does not copurify putative ND2 nascent chains. This suggests that ACAD9 acts downstream of MITRAC15, during the assembly of the $P_{P}-b$ module. Nevertheless, ACAD9 is crucial for the assembly of the $\mathrm{P}_{\mathrm{P}-\mathrm{b}}$ module, which is shown in Figure 39 and was also reported by Nouws et al. (Nouws et al., 2010). Unlike ACAD9, MITRAC15 is unlikely to play a role in $P_{P}-b$ module assembly. Despitecausing a decrease of ND2 synthesis by $44 \%$, the knockout of MITRAC15 did not lead to a decrease in complex I activity. Also it is debatable to which extent MITRAC15 stays associated with the assembling complex I. Guerrero Castillo et al. (Guerrero-Castillo et al., 2017) reported the presence of MITRAC15 and ACAD9 in late assembly intermediates up to $1 \mathrm{MDa}$. This could not be 
detected in the 2D analysis of isolated ACAD9 containing complexes presented in this thesis (Figure 35). Therefore, it is plausible that MITRAC15 may leave the assembling $\mathrm{P}_{\mathrm{P}}-\mathrm{b}$ module in its early stages. Furthermore, the stability of complex I activity, despite the $44 \%$ decrease of ND2 synthesis, is in itself an interesting phenomenon. Even though the electron transfer from $\mathrm{NADH}$ to ubiquinone, which was measured by the complex I activity assay (Figure 42), is performed by the $\mathrm{N}$ and $\mathrm{Q}$ module, Stroud et al. showed that the $\mathrm{P}_{\mathrm{P}}-\mathrm{b}$ module assembly is crucial for the stability of complex I. In the absence of the $\mathrm{P}_{\mathrm{P}-\mathrm{b}}$ module accessory subunits NDUFC1 or NDUFC2, complex I assembly cannot proceed(Stroud et al., 2016). Therefore, it is unlikely that complex I can assemble without the $\mathrm{P}_{\mathrm{P}}-\mathrm{b}$ module core subunit ND2. This suggests that $44 \%$ ND2 is enough to sustain WT levels of complex I activity.Figure 47 shows the revisited early assembly of the $\mathrm{P}_{\mathrm{P}}-\mathrm{b}$ module.

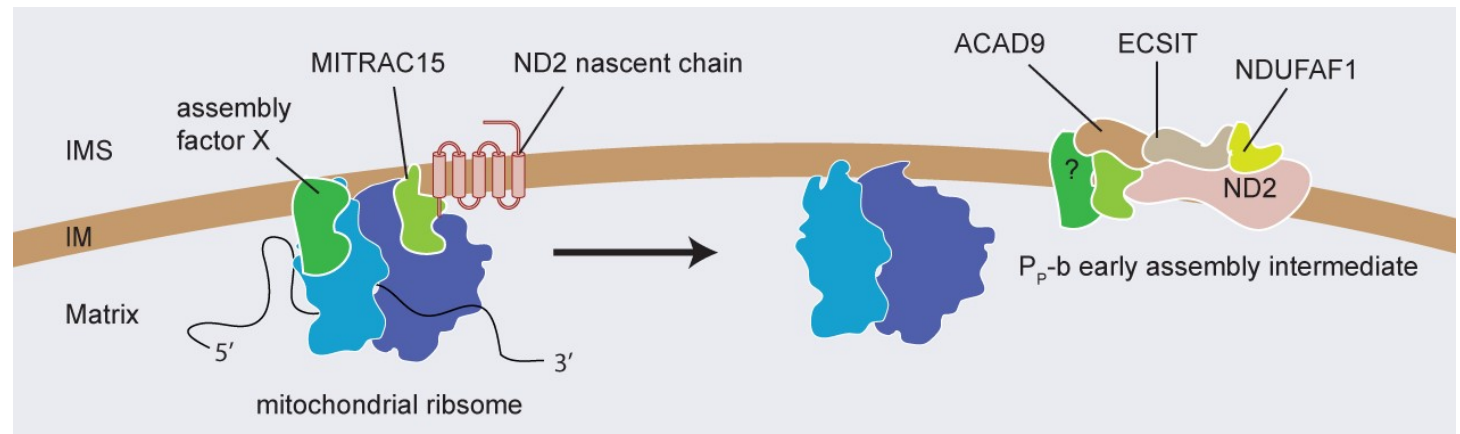

Figure 47 Early assembly of the $P_{\mathrm{p}}-\mathrm{b}$ module. MITRAC15 and possibly another assembly factor (assembly factor $X$ ) associates with the ND2 translating ribosome. After disassociation from the ribosome, ACAD9 joins other assembly factors promoting further assembly of the $\mathrm{P}_{\mathrm{P}}-\mathrm{b}$ module. Assembly factor $\mathrm{X}$ may or may not stay with the assembly intermediate.

Comparing OXPHOS assembly factors in yeast and human, some of the homologues still perform their conserved functions, like the heme $A$ insertases COX10 and COX15 (Soto et al., 2012;Antonicka, 2003;Antonicka et al., 2003). Other assembly factors, like Cox14/C12ORF62 and Coa3/MITRAC12, have diverged in their function from yeast to human, but still remain in the same assembly pathway (Mick et al., 2012; Richter-Dennerlein et al., 2016). In the case of MITRAC15, which shows a sequence similarity of $16.2 \%$ (determined with the Needleman-Wunsch algorithm (Needleman and Wunsch, 1970) with the BLOSUM62 matrix (Styczynski et al., 2008)) to its yeast counterpartCoa1, the changes are much more severe. Coa1 was 
characterized as an assembly factor which functions with Shy1, Mss51 and Cox14 in early to intermediate stages of complex IV assembly. Depletion of Coa1 causes strong defects in complex IV assembly and decreases the steady state levels of complex IV core subunits (Mick et al., 2007). MITRAC15 on the other hand, acts as a translation regulator and possibly also assembly factor in the assembly of complex I, a complex not existent in yeast. Furthermore, the function of MITRAC15 in complex IV assembly also changed from a crucial assembly factor to a factor stalling the complex IV assembly. Hence, MITRAC15 should not be characterized as a homologue of Coa1, but as a new human assembly factor, which is not present in yeast.

Nevertheless, the function and mechanisms of MITRAC15 need to be addressed in more detail to understand the complete role of this protein. 


\section{Summary and conclusion}

For an efficient assembly of those complexes, the translation in mitochondria and cytosol have to be coordinated. Mitochondrial translation regulation factors of cytosolic translational origin are an important component in the crosstalk between mitochondria and the cytosol. Despite many mitochondrial translation regulators are characterized in yeast, little is known about their role in human mitochondria. This work provided insight into the assembly of the early MITRAC complex during COX1 translation and established MITRAC15 as a translational regulator for the synthesis of the mitochondrial complex I core subunit ND2.

During this work, the mitochondrial ribosome associated form of the MITRAC complex (MITRAC ${ }^{\text {ribo }}$ ) could be isolated. MITRAC ${ }^{\text {ribo }}$ was analyzed by Western blot, mass spectrometry and cryoEM. The results obtained in the analysis show COX4I-1 as a subunit of MITRAC ribo. This implies that the early assembly of the cytochrome $c$ oxidase already starts during COX1 translation. Furthermore, MITRAC ${ }^{\text {ribo }}$ was could be localized near the exit tunnel of the mitochondrial ribosome and the insertase OXA1L could be identified as a subunit, which imply a role of MITRAC in coordinating the insertion of COX1 into the inner mitochondrial membrane. Crosslinking data has to be generated to provide insight into a more detailed composition of MITRAC ribo.

MITRAC15, a protein that act in both complex I and IV assembly, could be characterized more detailed in its function. MITRAC15 was shown to stall the assembly of the cytochrome $c$ oxidase, while it could be identified as a translation regulator important for the synthesis of ND2. Because MITRAC15 interacts with the mitochondrial ribosome, copurify nascent chains of mitochondrial synthesized proteins under puromycin treatment and show strong interaction with newly synthesized ND2, it is likely to associate with the ND2 translating mitochondrial ribosome. Finally, the complex I assembly factor ACAD9 could be shown to mediate the assembly of ND4L and ND3 into the $\mathrm{P}_{\mathrm{p}}-\mathrm{b}$ module. However, the mechanism how MITRAC15 functions still has to be investigated. 


\section{References}

Abe, Y., Shodai, T., Muto, T., Mihara, K., Torii, H., Nishikawa, S., Endo, T., and Kohda, D. (2000). Structural basis of presequence recognition by the mitochondrial protein import receptor Tom20. Cell 100, 551-560.

Abriata, L.A., Banci, L., Bertini, I., Ciofi-Baffoni, S., Gkazonis, P., Spyroulias, G.A., Vila, A.J., and Wang, S. (2008). Mechanism of $\mathrm{Cu}_{\mathrm{A}}$ assembly. Nat Chem Biol 4, 599-601.

Acin-Perez, R., Bayona-Bafaluy, M.P., Fernandez-Silva, P., MorenoLoshuertos, R., Pérez-Martos, A., Bruno, C., Moraes, C.T., and Enriquez, J.A. (2004). Respiratory complex III is required to maintain complex I in mammalian mitochondria. Molecular Cell 13, 805-815.

Agaronyan, K., Morozov, Y.I., Anikin, M., and Temiakov, D. (2015). Mitochondrial biology. Replication-transcription switch in human mitochondria. Science 347, 548-551.

Agip, A.-N.A., Blaza, J.N., Bridges, H.R., Viscomi, C., Rawson, S., Muench, S.P., and Hirst, J. (2018). Cryo-EM structures of complex I from mouse heart mitochondria in two biochemically defined states. Nature Publishing Group $1-$ 13.

Agris, P.F. (2008). Bringing order to translation: the contributions of transfer RNA anticodon-domain modifications. EMBO Rep 9, 629-635.

Aich, A., Wang, C., Chowdhury, A., Ronsör, C., Pacheu-Grau, D., RichterDennerlein, R., Dennerlein, S., and Rehling, P. (2018). COX16 promotes COX2 metallation and assembly during respiratory complex IV biogenesis. Elife 7, 34 .

Akabane, S., Ueda, T., Nierhaus, K.H., and Takeuchi, N. (2014). Ribosome Rescue and Translation Termination at Non-Standard Stop Codons by ICT1 in Mammalian Mitochondria. PLoS Genet 10, e1004616.

Altieri, D.C. (2012). Hsp90 regulation of mitochondrial protein folding: from organelle integrity to cellular homeostasis. Cellular and Molecular Life Sciences 70, 2463-2472.

Amunts, A., Brown, A., Bai, X.-C., Llácer, J.L., Hussain, T., Emsley, P., Long, F., Murshudov, G., Scheres, S.H.W., and Ramakrishnan, V. (2014). Structure of the yeast mitochondrial large ribosomal subunit. Science 343, 1485-1489.

Amunts, A., Brown, A., Toots, J., Scheres, S.H.W., and Ramakrishnan, V. (2015). Ribosome. The structure of the human mitochondrial ribosome. Science 348, 95-98.

Anderson, S., Bankier, A.T., Barrell, B.G., de Bruijn, M.H., Coulson, A.R., Drouin, J., Eperon, I.C., Nierlich, D.P., Roe, B.A., Sanger, F., et al. (1981). 
Sequence and organization of the human mitochondrial genome. Nature 290, 457-465.

Andrews, B., Carroll, J., Ding, S., Fearnley, I.M., and Walker, J.E. (2013). Assembly factors for the membrane arm of human complex I. Proc. Natl. Acad. Sci. U.S.a. 110, 18934-18939.

Angerer, H., Zwicker, K., Wumaier, Z., Sokolova, L., Heide, H., Steger, M., Kaiser, S., Nübel, E., Brutschy, B., Radermacher, M., et al. (2011). A scaffold of accessory subunits links the peripheral arm and the distal proton-pumping module of mitochondrial complex I. Biochem. J. 437, 279-288.

Ran, F.A., Hsu, P.D., Wright, J., Agarwala, V., Scott, D.A., and Zhang, F. (2013). Genome engineering using the CRISPR-Cas9 system. Nat Protoc 8, 2281-2308.

Antonicka, H. (2003). Mutations in COX10 result in a defect in mitochondrial heme $A$ biosynthesis and account for multiple, early-onset clinical phenotypes associated with isolated COX deficiency. Human Molecular Genetics 12, 2693-2702.

Antonicka, H., Mattman, A., Carlson, C.G., Glerum, D.M., Hoffbuhr, K.C., Leary, S.C., Kennaway, N.G., and Shoubridge, E.A. (2003). Mutations in COX15 produce a defect in the mitochondrial heme biosynthetic pathway, causing early-onset fatal hypertrophic cardiomyopathy. The American Journal of Human Genetics 72, 101-114.

Antonicka, H., Ostergaard, E., Sasarman, F., Weraarpachai, W., Wibrand, F., Pedersen, A.M.B., Rodenburg, R.J., van der Knaap, M.S., Smeitink, J.A.M., Chrzanowska-Lightowlers, Z.M., et al. (2010). REPOR T Mutations in C12orf65in Patients with Encephalomyopathy and a Mitochondrial Translation Defect. The American Journal of Human Genetics 87, 115-122.

Azzam, M.E., and Algranati, I.D. (1973). Mechanism of puromycin action: fate of ribosomes after release of nascent protein chains from polysomes. Proc Natl Acad Sci USA 70, 3866-3869.

Bai, X.-C., McMullan, G., and Scheres, S.H.W. (2015). How cryo-EM is revolutionizing structural biology. Trends in Biochemical Sciences 40, 49-57.

Banci, L., Bertini, I., Cantini, F., Ciofi-Baffoni, S., Gonnelli, L., and Mangani, S. (2004). Solution structure of Cox11, a novel type of beta-immunoglobulin-like fold involved in CuB site formation of cytochrome c oxidase. Journal of Biological Chemistry 279, 34833-34839.

Banci, L., Bertini, I., Ciofi-Baffoni, S., Gerothanassis, I.P., Leontari, I., Martinelli, M., and Wang, S. (2007a). A Structural Characterization of Human SCO2. Structure 15, 1132-1140.

Banci, L., Bertini, I., Ciofi-Baffoni, S., Hadjiloi, T., Martinelli, M., and Palumaa, P. (2008). Mitochondrial copper(I) transfer from Cox17 to Sco1 is coupled to electron transfer. Proc. Natl. Acad. Sci. U.S.a. 105, 6803-6808. 
Banci, L., Bertini, I., Ciofi-Baffoni, S., Leontari, I., Martinelli, M., Palumaa, P., Sillard, R., and Wang, S. (2007b). Human Sco1 functional studies and pathological implications of the P174L mutant. Proc Natl Acad Sci USA 104, 15-20.

Beers, J., Glerum, D.M., and Tzagoloff, A. (1997). Purification, characterization, and localization of yeast Cox17p, a mitochondrial copper shuttle. Journal of Biological Chemistry 272, 33191-33196.

Berk, A.J., and Clayton, D.A. (1974). Mechanism of mitochondrial DNA replication in mouse L-cells: asynchronous replication of strands, segregation of circular daughter molecules, aspects of topology and turnover of an initiation sequence. Journal of Molecular Biology 86, 801-824.

Berrisford, J.M., and Sazanov, L.A. (2009). Structural basis for the mechanism of respiratory complex I. J. Biol. Chem. 284, 29773-29783.

Berry, E.A., Guergova-Kuras, M., Huang, L.S., and Crofts, A.R. (2000). Structure and function of cytochrome bc complexes. Annu. Rev. Biochem. 69, 1005-1075.

Bhargava, K., Templeton, P., and Spremulli, L.L. (2004). Expression and characterization of isoform 1 of human mitochondrial elongation factor $\mathrm{G}$. Protein Expression and Purification 37, 368-376.

Bianchi, C., Genova, M.L., Parenti Castelli, G., and Lenaz, G. (2004). The mitochondrial respiratory chain is partially organized in a supercomplex assembly: kinetic evidence using flux control analysis. Journal of Biological Chemistry 279, 36562-36569.

Bode, M., Woellhaf, M.W., Bohnert, M., van der Laan, M., Sommer, F., Jung, M., Zimmermann, R., Schroda, M., and Herrmann, J.M. (2015). Redoxregulated dynamic interplay between Cox19 and the copper-binding protein Cox11 in the intermembrane space of mitochondria facilitates biogenesis of cytochrome c oxidase. Mol. Biol. Cell 26, 2385-2401.

Bogenhagen, D.F. (2012). Mitochondrial DNA nucleoid structure. BBA - Gene Regulatory Mechanisms 1819, 914-920.

Bonnefoy, N., Kermorgant, M., Groudinsky, O., Minet, M., Slonimski, P.P., and Dujardin, G. (1994). Cloning of a human gene involved in cytochrome oxidase assembly by functional complementation of an oxa1- mutation in Saccharomyces cerevisiae. Proc Natl Acad Sci USA 91, 11978-11982.

Bonnefoy, N., Fiumera, H.L., Dujardin, G., and Fox, T.D. (2009). Roles of Oxa1-related inner-membrane translocases in assembly of respiratory chain complexes. BBA - Molecular Cell Research 1793, 60-70.

Boos, F., Wollin, M., and Herrmann, J.M. (2016). Methionine on the rise: how mitochondria changed their codon usage. Embo J. 35, 2066-2067.

Borowski, L.S., Dziembowski, A., Hejnowicz, M.S., Stepien, P.P., and 
Szczesny, R.J. (2012). Human mitochondrial RNA decay mediated by PNPase-hSuv3 complex takes place in distinct foci. Nucleic Acids Research $41,1223-1240$.

Borst, P., and Grivell, L.A. (1971). Mitochondrial ribosomes. FEBS Letters 13, 73-88.

Botelho, S.E.C., sterberg, M.O., Reichert, A.S., Yamano, K., rkholm, P.B.O., Endo, T., Heijne, von, G., and Kim, H. (2011). TIM23-mediated insertion of transmembrane \&alpha;-helices into the mitochondrial inner membrane. Embo J. 30, 1003-1011.

Bourens, M., and Barrientos, A. (2017a). A CMC1-knockout reveals translation-independent control of human mitochondrial complex IV biogenesis. EMBO Rep 18, 477-494.

Bourens, M., and Barrientos, A. (2017b). Human mitochondrial cytochrome coxidase assembly factor COX18 acts transiently as a membrane insertase within the subunit 2 maturation module. Journal of Biological Chemistry 292, 7774-7783.

Bourens, M., Boulet, A., Leary, S.C., and Barrientos, A. (2014). Human COX20 cooperates with SCO1 and SCO2 to mature COX2 and promote the assembly of cytochrome c oxidase. Human Molecular Genetics 23, 29012913.

Bradford, M.M. (1976). A rapid and sensitive method for the quantitation of microgram quantities of protein utilizing the principle of protein-dye binding. Anal. Biochem. 72, 248-254.

Brandt, U. (2006). Energy converting NADH:quinone oxidoreductase (complex I). Annu. Rev. Biochem. 75, 69-92.

Brändén, M., Tomson, F., Gennis, R.B., and Brzezinski, P. (2002). The entry point of the K-proton-transfer pathway in cytochrome $c$ oxidase. Biochemistry 41, 10794-10798.

Brix, J., Ziegler, G.A., Dietmeier, K., Schneider-Mergener, J., Schulz, G.E., and Pfanner, N. (2000). The mitochondrial import receptor Tom70: identification of a $25 \mathrm{kda}$ core domain with a specific binding site for preproteins 1 1Edited by M. Yaniv. Journal of Molecular Biology 303, 479_ 488.

Brown, A., Amunts, A., Bai, X.-C., Sugimoto, Y., Edwards, P.C., Murshudov, G., Scheres, S.H.W., and Ramakrishnan, V. (2014). Structure of the large ribosomal subunit from human mitochondria. Science 346, 718-722.

Brzezniak, L.K., Bijata, M., Szczesny, R.J., and Stepien, P.P. (2014). Involvement of human ELAC2 gene product in 3' end processing of mitochondrial tRNAs. RNA Biology 8, 616-626.

Burger, G., Gray, M.W., and Franz Lang, B. (2003). Mitochondrial genomes: 
anything goes. Trends in Genetics 19, 709-716.

Bych, K., Netz, D.J.A., Vigani, G., Bill, E., Lill, R., Pierik, A.J., and Balk, J. (2008). The essential cytosolic iron-sulfur protein Nbp35 acts without Cfd1 partner in the green lineage. Journal of Biological Chemistry 283, 3579735804.

Callegari, S., Richter, F., Chojnacka, K., Jans, D.C., Lorenzi, I., Pacheu-Grau, D., Jakobs, S., Lenz, C., Urlaub, H., Dudek, J., et al. (2016). TIM29 is a subunit of the human carrier translocase required for protein transport. FEBS Letters 590, 4147-4158.

Cardenas-Rodriguez, M., Chatzi, A., and Tokatlidis, K. (2018). Iron-sulfur clusters: from metals through mitochondria biogenesis to disease. JBIC Journal of Biological Inorganic Chemistry 1-12.

Carilla-Latorre, S., Gallardo, M.E., Annesley, S.J., Calvo-Garrido, J., Graña, O., Accari, S.L., Smith, P.K., Valencia, A., Garesse, R., Fisher, P.R., et al. (2010). MidA is a putative methyltransferase that is required for mitochondrial complex I function. Journal of Cell Science 123, 1674-1683.

Carr, H.S., George, G.N., and Winge, D.R. (2002). Yeast Cox11, a protein essential for cytochrome $c$ oxidase assembly, is a $\mathrm{Cu}(\mathrm{I})$-binding protein. Journal of Biological Chemistry 277, 31237-31242.

Castresana, J., Lübben, M., Saraste, M., and Higgins, D.G. (1994). Evolution of cytochrome oxidase, an enzyme older than atmospheric oxygen. Embo J. 13, 2516-2525.

Cecchini, G. (2003). Function and Structure of Complex II of the Respiratory Chain. Annu. Rev. Biochem. 72, 77-109.

Chacinska, A., Lind, M., Frazier, A.E., Dudek, J., Meisinger, C., Geissler, A., Sickmann, A., Meyer, H.E., Truscott, K.N., Guiard, B., et al. (2005).

Mitochondrial Presequence Translocase: Switching between TOM Tethering and Motor Recruitment Involves Tim21 and Tim17. 120, 817-829.

Chae, P.S., Rasmussen, S.G.F., Rana, R.R., Gotfryd, K., Chandra, R., Goren, M.A., Kruse, A.C., Nurva, S., Loland, C.J., Pierre, Y., et al. (2010). Maltoseneopentyl glycol (MNG) amphiphiles for solubilization, stabilization and crystallization of membrane proteins. Nat Meth 7, 1003-1008.

Chewawiwat, N., Yano, M., Terada, K., Hoogenraad, N.J., and Mori, M. (1999). Characterization of the novel mitochondrial protein import component, Tom34, in mammalian cells. J. Biochem. 125, 721-727.

Christian, B.E., and Spremulli, L.L. (2009). Evidence for an Active Role of IF3 $m$ tin the Initiation of Translation in Mammalian Mitochondria $†$. Biochemistry 48, 3269-3278.

Christian, B.E., and Spremulli, L.L. (2012). Mechanism of protein biosynthesis in mammalian mitochondria. Biochimica Et Biophysica Acta (BBA) - Gene 
Regulatory Mechanisms 1819, 1035-1054.

Chujo, T., Ohira, T., Sakaguchi, Y., Goshima, N., Nomura, N., Nagao, A., and Suzuki, T. (2012). LRPPRC/SLIRP suppresses PNPase-mediated mRNA decay and promotes polyadenylation in human mitochondria. Nucleic Acids Research 40, 8033-8047.

Couvillion, M.T., Soto, I.C., Shipkovenska, G., and Churchman, L.S. (2016). Synchronized mitochondrial and cytosolic translation programs. Nature 533, 499-503.

Cowling, V.H. (2009). Regulation of mRNA cap methylation. Biochem. J. 425, 295-302.

Cummings, H.S., and Hershey, J.W. (1994). Translation initiation factor IF1 is essential for cell viability in Escherichia coli. J. Bacteriol. 176, 198-205.

Dame, R.T., Espéli, O., Grainger, D.C., and Wiggins, P.A. (2012).

Multidisciplinary perspectives on bacterial genome organization and dynamics. Molecular Microbiology 86, 1023-1030.

de Lima Santos H, and Ciancaglini, P. (2000). A practical approach to the choice of a suitable detergent and optimal conditions for solubilizing a membrane protein. Biochem. Educ. 28, 178-182.

Denkert, N., Schendzielorz, A.B., Barbot, M., Versemann, L., Richter, F., Rehling, P., and Meinecke, M. (2017). Cation selectivity of the presequence translocase channel Tim23 is crucial for efficient protein import. Elife 6, 439.

Dennerlein, S., Oeljeklaus, S., Jans, D., Hellwig, C., Bareth, B., Jakobs, S., Deckers, M., Warscheid, B., and Rehling, P. (2015). MITRAC7 Acts as a COX1-Specific Chaperone and Reveals a Checkpoint during Cytochrome $c$ Oxidase Assembly. Cell Rep 12, 1644-1655.

Dennerlein, S., Wang, C., and Rehling, P. (2017). Plasticity of Mitochondrial Translation. Trends in Cell Biology 27, 712-721.

Desai, N., Brown, A., Amunts, A., and Ramakrishnan, V. (2017). The structure of the yeast mitochondrial ribosome. Science 355, 528-531.

Diaz, F., Fukui, H., Garcia, S., and Moraes, C.T. (2006). Cytochrome c oxidase is required for the assembly/stability of respiratory complex I in mouse fibroblasts. Molecular and Cellular Biology 26, 4872-4881.

Dinman, J.D. (2005). 5S rRNA: Structure and Function from Head to Toe. Int J Biomed Sci 1, 2-7.

Dunning, C.J.R., McKenzie, M., Sugiana, C., Lazarou, M., Silke, J., Connelly, A., Fletcher, J.M., Kirby, D.M., Thorburn, D.R., and Ryan, M.T. (2007). Human CIA30 is involved in the early assembly of mitochondrial complex I and mutations in its gene cause disease. Embo J. 26, 3227-3237. 
Eaton, S., Bartlett, K., and Pourfarzam, M. (1996). Mammalian mitochondrial beta-oxidation. Biochem. J. 320 ( Pt 2), 345-357.

Eberly, S.L., Locklear, V., and Spremulli, L.L. (1985). Bovine mitochondrial ribosomes. Elongation factor specificity. Journal of Biological Chemistry 260, 8721-8725.

Englmeier, R., Pfeffer, S., and Förster, F. (2017). Structure of the Human Mitochondrial Ribosome Studied In Situ by Cryoelectron Tomography. Structure/Folding and Design 25, 1574-1581.e2.

Fearnley, I.M., and Walker, J.E. (2002). Initiation codons in mammalian mitochondria: differences in genetic code in the organelle. Biochemistry 26, 8247-8251.

Fiedorczuk, K., Letts, J.A., Degliesposti, G., Kaszuba, K., Skehel, M., and Sazanov, L.A. (2016). Atomic structure of the entire mammalian mitochondrial complex I. Nature 538, 406-410.

Formosa, L.E., Mimaki, M., Frazier, A.E., McKenzie, M., Stait, T.L., Thorburn, D.R., Stroud, D.A., and Ryan, M.T. (2015). Characterization of mitochondrial FOXRED1 in the assembly of respiratory chain complex I. Human Molecular Genetics 24, 2952-2965.

Frey, T.G., and Mannella, C.A. (2000). The internal structure of mitochondria. Trends in Biochemical Sciences 25, 319-324.

Freyer, C., Cree, L.M., Mourier, A., Stewart, J.B., Koolmeister, C., Milenkovic, D., Wai, T., Floros, V.I., Hagström, E., Chatzidaki, E.E., et al. (2012). Variation in germline mtDNA heteroplasmy is determined prenatally but modified during subsequent transmission. Nature Genetics 44, 1282-1285.

Fu, Q., Li, H., Moorjani, P., Jay, F., Slepchenko, S.M., Bondarev, A.A., Johnson, P.L.F., Aximu-Petri, A., Prüfer, K., de Filippo, C., et al. (2014). Genome sequence of a 45,000-year-old modern human from western Siberia. Nature 514, 445-449.

Galkin, A.S., Grivennikova, V.G., and Vinogradov, A.D. (1999). -->H+/2estoichiometry in NADH-quinone reductase reactions catalyzed by bovine heart submitochondrial particles. FEBS Letters 451, 157-161.

Gallagher, S., Winston tank transfer systems, S.E., Fuller tank transfer systems, S.A., and Hurrell tank transfer systems; rev, J.G.R. (2001). Immunoblotting and Immunodetection (Hoboken, NJ, USA: John Wiley \& Sons, Inc.).

Gerards, M., Sallevelt, S.C.E.H., and Smeets, H.J.M. (2016). Leigh syndrome: Resolving the clinical and genetic heterogeneity paves the way for treatment options. Molecular Genetics and Metabolism 117, 300-312.

Giachin, G., Bouverot, R., Acajjaoui, S., Pantalone, S., and Soler-López, M. (2016). Dynamics of Human Mitochondrial Complex I Assembly: Implications 
for Neurodegenerative Diseases. Front. Mol. Biosci. 3, 767.

Glerum, D.M., Shtanko, A., and Tzagoloff, A. (1996). Characterization of COX17, a yeast gene involved in copper metabolism and assembly of cytochrome oxidase. Journal of Biological Chemistry 271, 14504-14509.

Gorbikova, E.A., Belevich, I., Wikström, M., and Verkhovsky, M.I. (2008). The proton donor for $\mathrm{O}-\mathrm{O}$ bond scission by cytochrome $c$ oxidase. Proc. Natl. Acad. Sci. U.S.a. 105, 10733-10737.

Greber, B.J., and Ban, N. (2016). Structure and Function of the Mitochondrial Ribosome. Annu. Rev. Biochem. 85, 103-132.

Greber, B.J., Bieri, P., Leibundgut, M., Leitner, A., Aebersold, R., Boehringer, D., and Ban, N. (2015a). Ribosome. The complete structure of the 55S mammalian mitochondrial ribosome. Science 348, 303-308.

Greber, B.J., Boehringer, D., Leibundgut, M., Bieri, P., Leitner, A., Schmitz, N., Aebersold, R., and Ban, N. (2014). The complete structure of the large subunit of the mammalian mitochondrial ribosome. Nature.

Greber, B.J., Boehringer, D., Leibundgut, M., Bieri, P., Leitner, A., Schmitz, N., Aebersold, R., and Ban, N. (2015b). The complete structure of the large subunit of the mammalian mitochondrial ribosome. Nature 515, 283-286.

Green-Willms, N.S., Butler, C.A., Dunstan, H.M., and Fox, T.D. (2001). Pet111p, an Inner Membrane-bound Translational Activator That Limits Expression of the Saccharomyces cerevisiaeMitochondrial Gene COX2. Journal of Biological Chemistry 276, 6392-6397.

Gualerzi, C.O., and Pon, C.L. (2015). Initiation of mRNA translation in bacteria: structural and dynamic aspects. Cellular and Molecular Life Sciences 72, 4341-4367.

Guarani, V., Paulo, J., Zhai, B., Huttlin, E.L., Gygi, S.P., and Harper, J.W. (2014). TIMMDC1/C3orf1 functions as a membrane-embedded mitochondrial complex I assembly factor through association with the MCIA complex. Molecular and Cellular Biology 34, 847-861.

Guerrero-Castillo, S., Baertling, F., Kownatzki, D., Wessels, H.J., Arnold, S., Brandt, U., and Nijtmans, L. (2017). The Assembly Pathway of Mitochondrial Respiratory Chain Complex I. Cell Metab. 25, 128-139.

Gustafsson, C.M., Falkenberg, M., and Larsson, N.-G. (2016). Maintenance and Expression of Mammalian Mitochondrial DNA. Annu. Rev. Biochem. 85, 133-160.

Hahn, A., Parey, K., Bublitz, M., Mills, D.J., Zickermann, V., Vonck, J., Kühlbrandt, W., and Meier, T. (2016). Structure of a Complete ATP Synthase Dimer Reveals the Molecular Basis of Inner Mitochondrial Membrane Morphology. Molecular Cell 63, 445-456. 
Haltia, T., Saraste, M., and Wikström, M. (1991). Subunit III of cytochrome $c$ oxidase is not involved in proton translocation: a site-directed mutagenesis study. Embo J. 10, 2015-2021.

Hanahan, D. (1983). Studies on transformation of Escherichia coli with plasmids. Journal of Molecular Biology 166, 557-580.

Hauswirth, W.W., and Laipis, P.J. (1982). Mitochondrial DNA polymorphism in a maternal lineage of Holstein cows. Proc Natl Acad Sci USA 79, 4686-4690.

Hällberg, B.M., and Larsson, N.-G. (2014). Making proteins in the powerhouse. Cell Metab. 20, 226-240.

He, M., Rutledge, S.L., Kelly, D.R., Palmer, C.A., Murdoch, G., Majumder, N., Nicholls, R.D., Pei, Z., Watkins, P.A., and Vockley, J. (2007). A New Genetic Disorder in Mitochondrial Fatty Acid $\beta$-Oxidation: ACAD9 Deficiency. The American Journal of Human Genetics 81, 87-103.

Hedges, S.B., Blair, J.E., Venturi, M.L., and Shoe, J.L. (2004). A molecular timescale of eukaryote evolution and the rise of complex multicellular life. BMC Evol. Biol. 4, 2.

Heijne, von, G. (1986). Why mitochondria need a genome. FEBS Letters 198, $1-4$.

Hell, K., Neupert, W., and Stuart, R.A. (2001). Oxa1p acts as a general membrane insertion machinery for proteins encoded by mitochondrial DNA. Embo J. 20, 1281-1288.

Helm, M., Brulé, H., Friede, D., Giegé, R., Pütz, D., and Florentz, C. (2000). Search for characteristic structural features of mammalian mitochondrial tRNAs. Rna 6, 1356-1379.

Hiser, L., Di Valentin, M., Hamer, A.G., and Hosler, J.P. (2000). Cox11p is required for stable formation of the $\mathrm{Cu}(\mathrm{B})$ and magnesium centers of cytochrome $c$ oxidase. Journal of Biological Chemistry 275, 619-623.

Horst, M., Oppliger, W., Rospert, S., Schönfeld, H.J., Schatz, G., and Azem, A. (1997). Sequential action of two hsp70 complexes during protein import into mitochondria. Embo J. 16, 1842-1849.

Hovers, J., Potschies, M., Polidori, A., Pucci, B., Raynal, S., Bonneté, F., Serrano-Vega, M.J., Tate, C.G., Picot, D., Pierre, Y., et al. (2011). A class of mild surfactants that keep integral membrane proteins water-soluble for functional studies and crystallization. Molecular Membrane Biology 28, 171181.

Hsu, P.D., Scott, D.A., Weinstein, J.A., Ran, F.A., Konermann, S., Agarwala, V., Li, Y., Fine, E.J., Wu, X., Shalem, O., et al. (2013). DNA targeting specificity of RNA-guided Cas9 nucleases. Nature Biotechnology 31, 827832. 
Huynen, M.A., Duarte, I., Chrzanowska-Lightowlers, Z.M.A., and Nabuurs, S.B. (2012). Structure based hypothesis of a mitochondrial ribosome rescue mechanism. Biol. Direct 7, 14.

Hüttemann, M., Kadenbach, B., and Grossman, L.I. (2001). Mammalian subunit IV isoforms of cytochrome $c$ oxidase. Gene 267, 111-123.

leva, R., Schrempp, S.G., Opaliński, Ł., Wollweber, F., Höß, P., Heißwolf, A.K., Gebert, M., Zhang, Y., Guiard, B., Rospert, S., et al. (2014). Mgr2 Functions as Lateral Gatekeeper for Preprotein Sorting in the Mitochondrial Inner Membrane. Molecular Cell 56, 641-652.

Jan, P.S., Esser, K., Pratje, E., and Michaelis, G. (2000). Som1, a third component of the yeast mitochondrial inner membrane peptidase complex that contains Imp1 and Imp2. Mol. Gen. Genet. 263, 483-491.

Jeffreys, A.J., and Craig, I.W. (1976). Analysis of proteins synthesized in mitochondria of cultured mammalian cells. An assessment of current approaches and problems in interpretation. Eur. J. Biochem. 68, 301-311.

Jones, C.N., Wilkinson, K.A., Hung, K.T., Weeks, K.M., and Spremulli, L.L. (2008). Lack of secondary structure characterizes the 5' ends of mammalian mitochondrial mRNAs. Rna 14, 862-871.

Jores, T., Klinger, A., szlig, L.E.G., Kawano, S., Flinner, N., Duchardt-Ferner, E., hnert, J.W.O., Kalbacher, H., Endo, T., Schleiff, E., et al. (2016).

Characterization of the targeting signal in mitochondrial \&beta;-barrel proteins. Nature Communications 7, 1-16.

Jourdain, A.A., Koppen, M., Wydro, M., Rodley, C.D., Lightowlers, R.N., Chrzanowska-Lightowlers, Z.M., and Martinou, J.-C. (2013). GRSF1

Regulates RNA Processing in Mitochondrial RNA Granules. Cell Metab. 17, 399-410.

Kaila, V.R.I., Verkhovsky, M.I., and Wikström, M. (2010). Proton-Coupled Electron Transfer in Cytochrome Oxidase. Chem. Rev. 110, 7062-7081.

Kaneda, H., Hayashi, J., Takahama, S., Taya, C., Lindahl, K.F., and Yonekawa, H. (1995). Elimination of paternal mitochondrial DNA in intraspecific crosses during early mouse embryogenesis. Proc Natl Acad Sci USA 92, 4542-4546.

Kang, Y., Baker, M.J., Liem, M., Louber, J., McKenzie, M., Atukorala, I., Ang, C.-S., Keerthikumar, S., Mathivanan, S., and Stojanovski, D. (2016). Tim29 is a novel subunit of the human TIM22 translocase and is involved in complex assembly and stability. Elife 5,313 .

Kang, Y., Fielden, L.F., and Stojanovski, D. (2017). Mitochondrial protein transport in health and disease. Seminars in Cell and Developmental Biology $1-12$.

Kato, H., and Mihara, K. (2008). Identification of Tom5 and Tom6 in the 
preprotein translocase complex of human mitochondrial outer membrane. Biochemical and Biophysical Research Communications 369, 958-963.

Kevelam, S.H., Rodenburg, R.J., Wolf, N.I., Ferreira, P., Lunsing, R.J., Nijtmans, L.G., Mitchell, A., Arroyo, H.A., Rating, D., Vanderver, A., et al. (2013). NUBPL mutations in patients with complex I deficiency and a distinct MRI pattern. Neurology 80, 1577-1583.

Khalimonchuk, O., Kim, H., Watts, T., Perez-Martinez, X., and Winge, D.R. (2012). Oligomerization of heme o synthase in cytochrome oxidase biogenesis is mediated by cytochrome oxidase assembly factor Coa2. J. Biol. Chem. 287, 26715-26726.

Klein, A., Israel, L., Lackey, S.W.K., Nargang, F.E., Imhof, A., Baumeister, W., Neupert, W., and Thomas, D.R. (2012). Characterization of the insertase for $\beta$-barrel proteins of the outer mitochondrial membrane. The Journal of Cell Biology 199, 599-611.

Kmita, K., Wirth, C., Warnau, J., Guerrero-Castillo, S., Hunte, C., Hummer, G., Kaila, V.R.I., Zwicker, K., Brandt, U., and Zickermann, V. (2015). Accessory NUMM (NDUFS6) subunit harbors a Zn-binding site and is essential for biogenesis of mitochondrial complex I. Proc. Natl. Acad. Sci. U.S.a. 112, 5685-5690.

Koc, E.C., and Spremulli, L.L. (2002). Identification of mammalian mitochondrial translational initiation factor 3 and examination of its role in initiation complex formation with natural mRNAs. Journal of Biological Chemistry $277,35541-35549$.

Koch, M., Clementi, N., Rusca, N., Vögele, P., Erlacher, M., and Polacek, N. (2015). The integrity of the G2421-C2395 base pair in the ribosomal E-site is crucial for protein synthesis. RNA Biology 12, 70-81.

Kopp, E., Medzhitov, R., Carothers, J., Xiao, C., Douglas, I., Janeway, C.A., and Ghosh, S. (1999). ECSIT is an evolutionarily conserved intermediate in the Toll/IL-1 signal transduction pathway. Genes \& Development 13, 20592071.

Korkmaz, G., Holm, M., Wiens, T., and Sanyal, S. (2014). Comprehensive analysis of stop codon usage in bacteria and its correlation with release factor abundance. J. Biol. Chem. 289, 30334-30342.

Kukat, C., and Larsson, N.-G. (2013). mtDNA makes a U-turn for the mitochondrial nucleoid. Trends in Cell Biology 23, 457-463.

Kukat, C., Wurm, C.A., Spåhr, H., Falkenberg, M., Larsson, N.-G., and Jakobs, S. (2011). Super-resolution microscopy reveals that mammalian mitochondrial nucleoids have a uniform size and frequently contain a single copy of mtDNA. Proc. Natl. Acad. Sci. U.S.a. 108, 13534-13539.

Kurata, S., Ohtsuki, T., Wada, T., Kirino, Y., Takai, K., Saigo, K., Watanabe, K., and Suzuki, T. (2003). Decoding property of C5 uridine modification at the 
wobble position of tRNA anticodon. Nucleic Acids Res. Suppl. 245-246.

Laemmli, U.K. (1970). Cleavage of structural proteins during the assembly of the head of bacteriophage T4. Nature 227, 680-685.

Lazarou, M., McKenzie, M., Ohtake, A., Thorburn, D.R., and Ryan, M.T. (2007). Analysis of the assembly profiles for mitochondrial- and nuclear-DNAencoded subunits into complex I. Molecular and Cellular Biology 27, 42284237.

Lazarou, M., Smith, S.M., Thorburn, D.R., Ryan, M.T., and McKenzie, M. (2009). Assembly of nuclear DNA-encoded subunits into mitochondrial complex IV, and their preferential integration into supercomplex forms in patient mitochondria. FEBS Journal 276, 6701-6713.

Leary, S.C., Sasarman, F., Nishimura, T., and Shoubridge, E.A. (2009). Human SCO2 is required for the synthesis of $\mathrm{CO}$ II and as a thiol-disulphide oxidoreductase for SCO1. Human Molecular Genetics 18, 2230-2240.

Lee, C.C., Timms, K.M., Trotman, C.N., and Tate, W.P. (1987). Isolation of a rat mitochondrial release factor. Accommodation of the changed genetic code for termination. Journal of Biological Chemistry 262, 3548-3552.

Lerma, R.A., Tidwell, T.J., Cahill, J.L., Rasche, E.S., and Kuty Everett, G.F. (2015). Complete Genome Sequence of Caulobacter crescentus Podophage Percy. Genome Announc. 3, e01373-15.

Letts, J.A., and Sazanov, L.A. (2017). Clarifying the supercomplex: the higherorder organization of the mitochondrial electron transport chain. Nat Struct Mol Biol 24, 800-808.

Liao, H.X., and Spremulli, L.L. (1990). Identification and initial characterization of translational initiation factor 2 from bovine mitochondria. Journal of Biological Chemistry 265, 13618-13622.

Lind, C., Sund, J., and qvist, J.A. (2013). Codon-reading specificities of mitochondrial release factors and translation termination at non-standard stop codons. Nature Communications 4, 1-8.

Lopez-Fabuel, I., Le Douce, J., Logan, A., James, A.M., Bonvento, G., Murphy, M.P., Almeida, A., and Bolaños, J.P. (2016). Complex I assembly into supercomplexes determines differential mitochondrial ROS production in neurons and astrocytes. Proc. Natl. Acad. Sci. U.S.a. 113, 13063-13068.

Lorenzi, I., Oeljeklaus, S., Aich, A., Ronsör, C., Callegari, S., Dudek, J., Warscheid, B., Dennerlein, S., and Rehling, P. (2018). The mitochondrial TMEM177 associates with COX20 during COX2 biogenesis. BBA - Molecular Cell Research 1865, 323-333.

Lorenzi, I., Oeljeklaus, S., Ronsör, C., Bareth, B., Warscheid, B., Rehling, P., and Dennerlein, S. (2016). The ribosome-associated Mba1 escorts Cox2 from insertion machinery to maturing assembly intermediates. Molecular and 
Cellular Biology 36, 2782-2793.

Malarkey, C.S., and Churchill, M.E.A. (2012). The high mobility group box: the ultimate utility player of a cell. Trends in Biochemical Sciences 37, 553-562.

Marchler-Bauer, A., Derbyshire, M.K., Gonzales, N.R., Lu, S., Chitsaz, F., Geer, L.Y., Geer, R.C., He, J., Gwadz, M., Hurwitz, D.I., et al. (2014). CDD: NCBI's conserved domain database. Nucleic Acids Research 43, D222D226.

Martin, A.P. (1995). Metabolic rate and directional nucleotide substitution in animal mitochondrial DNA. Mol. Biol. Evol. 12, 1124-1131.

Matta, S.K., Pareek, G., Bankapalli, K., Oblesha, A., and D'Silva, P. (2017). Role of Tim17 Transmembrane Regions in Regulating the Architecture of Presequence Translocase and Mitochondrial DNA Stability. Molecular and Cellular Biology 37.

Meisinger, C., Ryan, M.T., Hill, K., Model, K., Lim, J.H., Sickmann, A., Muller, H., Meyer, H.E., Wagner, R., and Pfanner, N. (2001). Protein import channel of the outer mitochondrial membrane: a highly stable Tom40-Tom22 core structure differentially interacts with preproteins, small tom proteins, and import receptors. Molecular and Cellular Biology 21, 2337-2348.

Meisinger, C., Sickmann, A., and Pfanner, N. (2008). The Mitochondrial Proteome: From Inventory to Function. Cell 134, 22-24.

Metodiev, M.D., Spåhr, H., Loguercio Polosa, P., Meharg, C., Becker, C., Altmueller, J., Habermann, B., Larsson, N.-G., and Ruzzenente, B. (2014). NSUN4 Is a Dual Function Mitochondrial Protein Required for Both Methylation of $12 \mathrm{~S}$ rRNA and Coordination of Mitoribosomal Assembly. PLoS Genet 10, e1004110.

Michel, H., Behr, J., Harrenga, A., and Kannt, A. (1998). Cytochrome c oxidase: structure and spectroscopy. Annu Rev Biophys Biomol Struct 27, 329-356.

Mick, D.U., Dennerlein, S., Wiese, H., Reinhold, R., Pacheu-Grau, D., Lorenzi, I., Sasarman, F., Weraarpachai, W., Shoubridge, E.A., Warscheid, B., et al. (2012). MITRAC links mitochondrial protein translocation to respiratory-chain assembly and translational regulation. Cell 151, 1528-1541.

Mick, D.U., Vukotic, M., Piechura, H., Meyer, H.E., Warscheid, B., Deckers, M., and Rehling, P. (2010). Coa3 and Cox14 are essential for negative feedback regulation of COX1translation in mitochondria. The Journal of Cell Biology 191, 141-154.

Mick, D.U., Wagner, K., van der Laan, M., Frazier, A.E., Perschil, I., Pawlas, M., Meyer, H.E., Warscheid, B., and Rehling, P. (2007). Shy1 couples Cox1 translational regulation to cytochrome $c$ oxidase assembly. Embo J. 26, 43474358. 
Minczuk, M., He, J., Duch, A.M., Ettema, T.J., Chlebowski, A., Dzionek, K., Nijtmans, L.G.J., Huynen, M.A., and Holt, I.J. (2011). TEFM (c17orf42) is necessary for transcription of human mtDNA. Nucleic Acids Research 39, 4284-4299.

Mitchell, P., and Moyle, J. (1968). Proton translocation coupled to ATP hydrolysis in rat liver mitochondria. Eur. J. Biochem. 4, 530-539.

Morgenstern, M., Stiller, S.B., Lübbert, P., Peikert, C.D., Dannenmaier, S., Drepper, F., Weill, U., Höß, P., Feuerstein, R., Gebert, M., et al. (2017).

Definition of a High-Confidence Mitochondrial Proteome at Quantitative Scale. Cell Rep 19, 2836-2852.

Mori, M., Miura, S., Morita, T., Takiguchi, M., and Tatibana, M. (1982).

Ornithine transcarbamylase in liver mitochondria. Mol. Cell. Biochem. 49, 97111.

Muller, H.J. (1964). Therelationofrecombinationtomutationaladvance. Mutat. Res. 106, 2-9.

Nagaike, T., Suzuki, T., Katoh, T., and Ueda, T. (2005). Human mitochondrial mRNAs are stabilized with polyadenylation regulated by mitochondria-specific poly(A) polymerase and polynucleotide phosphorylase. Journal of Biological Chemistry 280, 19721-19727.

Nass, M.M. (1966). The circularity of mitochondrial DNA. Proc Natl Acad Sci USA 56, 1215-1222.

Needleman, S.B., and Wunsch, C.D. (1970). A general method applicable to the search for similarities in the amino acid sequence of two proteins. Journal of Molecular Biology 48, 443-453.

Nierlich, D.P. (1982). Fragmentary 5S rRNA gene in the human mitochondrial genome. Molecular and Cellular Biology 2, 207-209.

Nouws, J., Brinke, te, H., Nijtmans, L.G., and Houten, S.M. (2013). ACAD9, a complex I assembly factor with a moonlighting function in fatty acid oxidation deficiencies. Human Molecular Genetics 23, 1311-1319.

Nouws, J., Nijtmans, L., Houten, S.M., van den Brand, M., Huynen, M., Venselaar, H., Hoefs, S., Gloerich, J., Kronick, J., Hutchin, T., et al. (2010). Acyl-CoA Dehydrogenase 9 Is Required for the Biogenesis of Oxidative Phosphorylation Complex I. Cell Metab. 12, 283-294.

Ogilvie, I., Kennaway, N.G., and Shoubridge, E.A. (2005). A molecular chaperone for mitochondrial complex I assembly is mutated in a progressive encephalopathy. Journal of Clinical Investigation 115, 2784-2792.

Ojala, D., Montoya, J., and Attardi, G. (1981). tRNA punctuation model of RNA processing in human mitochondria. Nature 290, 470-474.

Ostergaard, E., Rodenburg, R.J., van den Brand, M., Thomsen, L.L., Duno, 
M., Batbayli, M., Wibrand, F., and Nijtmans, L. (2011). Respiratory chain complex I deficiency due to NDUFA12 mutations as a new cause of Leigh syndrome. J. Med. Genet. 48, 737-740.

Oswald, C., Krause-Buchholz, U., and Rödel, G. (2009). Knockdown of Human COX17 Affects Assembly and Supramolecular Organization of Cytochrome $c$ Oxidase. Journal of Molecular Biology 389, 470-479.

Ott, M., Amunts, A., and Brown, A. (2015). Organization and Regulation of Mitochondrial Protein Synthesis. Annu. Rev. Biochem. 85, annurev-biochem060815-014334.

Ott, M., Prestele, M., Bauerschmitt, H., Funes, S., Bonnefoy, N., and Herrmann, J.M. (2006). Mba1, a membrane-associated ribosome receptor in mitochondria. Embo J. 25, 1603-1610.

Pacheu-Grau, D., Bareth, B., Dudek, J., Juris, L., VOgtle, F.N., Wissel, M., Leary, S.C., Dennerlein, S., Rehling, P., and Deckers, M. (2015). Cooperation between $\mathrm{COA} 6$ and $\mathrm{SCO} 2$ in $\mathrm{COX} 2$ Maturation during Cytochrome $c$ Oxidase Assembly Links Two Mitochondrial Cardiomyopathies. Cell Metab. 21, 823833.

Palmfeldt, J., and Bross, P. (2017). Proteomics of human mitochondria. Mitochondrion 33, 2-14.

Panov, A. (2013). Practical Mitochondriology. Createspace Independent Pub.

Park, C.B., Asin-Cayuela, J., Cámara, Y., Shi, Y., Pellegrini, M., Gaspari, M., Wibom, R., Hultenby, K., Erdjument-Bromage, H., Tempst, P., et al. (2007). MTERF3 Is a Negative Regulator of Mammalian mtDNA Transcription. Cell 130, 273-285.

Park, K., Botelho, S.C., Hong, J., Österberg, M., and Kim, H. (2013). Dissecting stop transfer versus conservative sorting pathways for mitochondrial inner membrane proteins in vivo. J. Biol. Chem. 288, $1521-$ 1532.

Pathak, B.K., Mondal, S., and Barat, C. (2016). Inhibition of Escherichia coliribosome subunit dissociation by chloramphenicol and Blasticidin: a new mode of action of the antibiotics. Lett Appl Microbiol 64, 79-85.

Pellegrini, M., Asin-Cayuela, J., Erdjument-Bromage, H., Tempst, P., Larsson, N.-G., and Gustafsson, C.M. (2009). MTERF2 is a nucleoid component in mammalian mitochondria. BBA - Bioenergetics 1787, 296-302.

Perales-Clemente, E., Fernandez-Vizarra, E., Acin-Perez, R., Movilla, N., Bayona-Bafaluy, M.P., Moreno-Loshuertos, R., Pérez-Martos, A., FernandezSilva, P., and Enríquez, J.A. (2010). Five entry points of the mitochondrially encoded subunits in mammalian complex I assembly. Molecular and Cellular Biology 30, 3038-3047.

Peralta, S., Clemente, P., Sánchez-Martínez, Á., Calleja, M., Hernández- 
Sierra, R., Matsushima, Y., Adán, C., Ugalde, C., Fernández-Moreno, M.Á., Kaguni, L.S., et al. (2012). Coiled Coil Domain-containing Protein 56 (CCDC56) Is a Novel Mitochondrial Protein Essential for Cytochrome cOxidase Function. Journal of Biological Chemistry 287, 24174-24185.

Pfisterer, J., and Buetow, D.E. (1981). In vitro reconstruction of the mitochondrial translation system of yeast. Proc Natl Acad Sci USA 78, 49174921.

Pierrel, F., Bestwick, M.L., Cobine, P.A., Khalimonchuk, O., Cricco, J.A., and Winge, D.R. (2007). Coa1 links the Mss51 post-translational function to Cox1 cofactor insertion in cytochrome $c$ oxidase assembly. Embo J. 26, 4335-4346.

Posse, V., Hoberg, E., Dierckx, A., Shahzad, S., Koolmeister, C., Larsson, N.G., Wilhelmsson, L.M., Hällberg, B.M., and Gustafsson, C.M. (2014). The amino terminal extension of mammalian mitochondrial RNA polymerase ensures promoter specific transcription initiation. Nucleic Acids Research 42, 3638-3647.

Posse, V., Shahzad, S., Falkenberg, M., Hällberg, B.M., and Gustafsson, C.M. (2015). TEFM is a potent stimulator of mitochondrial transcription elongation in vitro. Nucleic Acids Research 43, 2615-2624.

Rainbolt, T.K., Atanassova, N., Genereux, J.C., and Wiseman, R.L. (2013). Stress-Regulated Translational Attenuation Adapts Mitochondrial Protein Import through Tim17A Degradation. Cell Metab. 18, 908-919.

Ray-Soni, A., Bellecourt, M.J., and Landick, R. (2016). Mechanisms of Bacterial Transcription Termination: All Good Things Must End. Annu. Rev. Biochem. 85, 319-347.

Rehling, P., Brandner, K., and Pfanner, N. (2004). Mitochondrial import and the twin-pore translocase. Nat Rev Mol Cell Biol 5, 519-530.

Reinhard, L., Sridhara, S., and Hällberg, B.M. (2017). The MRPP1/MRPP2 complex is a tRNA-maturation platform in human mitochondria. Nucleic Acids Research 45, 12469-12480.

Rhein, V.F., Carroll, J., Ding, S., Fearnley, I.M., and Walker, J.E. (2013). NDUFAF7 methylates arginine 85 in the NDUFS2 subunit of human complex I. J. Biol. Chem. 288, 33016-33026.

Rhein, V.F., Carroll, J., Ding, S., Fearnley, I.M., and Walker, J.E. (2016). NDUFAF5 Hydroxylates NDUFS7 at an Early Stage in the Assembly of Human Complex I. J. Biol. Chem. 291, 14851-14860.

Richman, T.R., hr, H.S.A., Ermer, J.A., Davies, S.M.K., Viola, H.M., Bates, K.A., Papadimitriou, J., Hool, L.C., Rodger, J., Larsson, N.-G.O.R., et al. (2016). Loss of the RNA-binding protein TACO1 causes late-onset mitochondrial dysfunction in mice. Nature Communications 7, 1-14.

Richter, R., Rorbach, J., Pajak, A., Smith, P.M., Wessels, H.J., Huynen, M.A., 
Smeitink, J.A., Lightowlers, R.N., and Chrzanowska-Lightowlers, Z.M. (2010). A functional peptidyl-tRNA hydrolase, ICT1, has been recruited into the human mitochondrial ribosome. Embo J. 29, 1116-1125.

Richter-Dennerlein, R., Oeljeklaus, S., Lorenzi, I., Ronsör, C., Bareth, B., Schendzielorz, A.B., Wang, C., Warscheid, B., Rehling, P., and Dennerlein, S. (2016). Mitochondrial Protein Synthesis Adapts to Influx of Nuclear-Encoded Protein. Cell 167, 471-483.e10.

Ringel, R., Sologub, M., Morozov, Y.I., Litonin, D., Cramer, P., and Temiakov, D. (2011). Structure of human mitochondrial RNA polymerase. Nature 478, 269-273.

Rizzuto, R., De Stefani, D., Raffaello, A., and Mammucari, C. (2012). Mitochondria as sensors and regulators of calcium signalling. Nat Rev Mol Cell Biol 13, 566-578.

Roise, D., Horvath, S.J., Tomich, J.M., Richards, J.H., and Schatz, G. (1986). A chemically synthesized pre-sequence of an imported mitochondrial protein can form an amphiphilic helix and perturb natural and artificial phospholipid bilayers. Embo J. 5, 1327-1334.

Rossmanith, J.H.P.F.E.L.K.L.B.C.G.W. (2008). RNase P without RNA: Identification and Functional Reconstitution of the Human Mitochondrial tRNA Processing Enzyme. Cell 135, 462-474.

Russell, S.A. (2000). Molecular Cloning: a laboratory manual 3rd Edition. (CSHL Press.).

Saada, A., Vogel, R.O., Hoefs, S.J., van den Brand, M.A., Wessels, H.J., Willems, P.H., Venselaar, H., Shaag, A., Barghuti, F., Reish, O., et al. (2009). AR TICLEMutations in NDUFAF3 (C3ORF60), Encoding an NDUFAF4 (C6ORF66)-Interacting Complex I Assembly Protein, Cause Fatal Neonatal Mitochondrial Disease. The American Journal of Human Genetics 84, 718727.

Sagan, L. (1967). On the origin of mitosing cells. J. Theor. Biol. 14, 255-274.

Saracco, S.A., and Fox, T.D. (2002). Cox18p is required for export of the mitochondrially encoded Saccharomyces cerevisiae Cox2p C-tail and interacts with Pnt1p and Mss2 $p$ in the inner membrane. Mol. Biol. Cell 13, 1122-1131.

Sazanov, L.A., Peak-Chew, S.Y., Fearnley, I.M., and Walker, J.E. (2000). Resolution of the Membrane Domain of Bovine Complex I into Subcomplexes: Implications for the Structural Organization of the Enzyme. Biochemistry 39, 7229-7235.

Sánchez-Caballero, L., Guerrero-Castillo, S., and Nijtmans, L. (2016). Unraveling the complexity of mitochondrial complex I assembly: A dynamic process. BBA - Bioenergetics 1857, 980-990. 
Schäfer, E., Seelert, H., Reifschneider, N.H., Krause, F., Dencher, N.A., and Vonck, J. (2006). Architecture of active mammalian respiratory chain supercomplexes. Journal of Biological Chemistry 281, 15370-15375.

Schägger, H., and Jagow, von, G. (1991). Blue native electrophoresis for isolation of membrane protein complexes in enzymatically active form. Anal. Biochem. 199, 223-231.

Schägger, H., and Pfeiffer, K. (2000). Supercomplexes in the respiratory chains of yeast and mammalian mitochondria. Embo J. 19, 1777-1783.

Schägger, H. (2002). Respiratory chain supercomplexes of mitochondria and bacteria. Biochim. Biophys. Acta 1555, 154-159.

Schägger, H. (2006). Tricine-SDS-PAGE. Nat Protoc 1, 16-22.

Schiff, M., Haberberger, B., Xia, C., Mohsen, A.-W., Goetzman, E.S., Wang, Y., Uppala, R., Zhang, Y., Karunanidhi, A., Prabhu, D., et al. (2015). Complex I assembly function and fatty acid oxidation enzyme activity of ACAD9 both contribute to disease severity in ACAD9 deficiency. Human Molecular Genetics 24, 3238-3247.

Schlehe, J.S., Journel, M.S.M., Taylor, K.P., Amodeo, K.D., and LaVoie, M.J. (2013). The mitochondrial disease associated protein Ndufaf2 is dispensable for Complex-1 assembly but critical for the regulation of oxidative stress. Neurobiology of Disease 58, 57-67.

Schmitt, H. (1969). Characterization of mitochondrial ribosomes from Saccharomyces cerevisiae. FEBS Letters 4, 234-238.

Schulz, C., Lytovchenko, O., Melin, J., Chacinska, A., Guiard, B., Neumann, P., Ficner, R., Jahn, O., Schmidt, B., and Rehling, P. (2011). Tim50's presequence receptor domain is essential for signal driven transport across the TIM23 complex. The Journal of Cell Biology 195, 643-656.

Schulz, C., Schendzielorz, A., and Rehling, P. (2015). Unlocking the presequence import pathway. Trends in Cell Biology 25, 265-275.

Schwartzbach, C.J., and Spremulli, L.L. (1989). Bovine mitochondrial protein synthesis elongation factors. Identification and initial characterization of an elongation factor Tu-elongation factor Ts complex. Journal of Biological Chemistry 264, 19125-19131.

Schwinghammer, K., Cheung, A.C.M., Morozov, Y.I., Agaronyan, K., Temiakov, D., and Cramer, P. (2013). Structure of human mitochondrial RNA polymerase elongation complex. Nature Publishing Group 20, 1298-1303.

Sharma, M.R., Koc, E.C., Datta, P.P., Booth, T.M., Spremulli, L.L., and Agrawal, R.K. (2003). Structure of the mammalian mitochondrial ribosome reveals an expanded functional role for its component proteins. Cell 115, 97108. 
Sheftel, A.D., Stehling, O., Pierik, A.J., Netz, D.J.A., Kerscher, S., Elsässer, H.-P., Wittig, I., Balk, J., Brandt, U., and Lill, R. (2009). Human ind1, an ironsulfur cluster assembly factor for respiratory complex I. Molecular and Cellular Biology 29, 6059-6073.

Shiota, T., Imai, K., Qiu, J., Hewitt, V.L., Tan, K., Shen, H.-H., Sakiyama, N., Fukasawa, Y., Hayat, S., Kamiya, M., et al. (2015). Molecular architecture of the active mitochondrial protein gate. Science 349, 1544-1548.

Siira, S.J., Spåhr, H., Shearwood, A.-M.J., Ruzzenente, B., Larsson, N.-G., Rackham, O., and Filipovska, A. (2017). LRPPRC-mediated folding of the mitochondrial transcriptome. Nature Communications 1-10.

Sinha, D., Srivastava, S., Krishna, L., and D'Silva, P. (2014). Unraveling the intricate organization of mammalian mitochondrial presequence translocases: existence of multiple translocases for maintenance of mitochondrial function. Molecular and Cellular Biology 34, 1757-1775.

Soto, I.C., Fontanesi, F., Liu, J., and Barrientos, A. (2012). Biogenesis and assembly of eukaryotic cytochrome $c$ oxidase catalytic core. BBA Bioenergetics 1817, 883-897.

Spåhr, H., Rozanska, A., Li, X., Atanassov, I., Lightowlers, R.N., Chrzanowska-Lightowlers, Z.M.A., Rackham, O., and Larsson, N.-G. (2016). SLIRP stabilizes LRPPRC via an RRM-PPR protein interface. Nucleic Acids Research 44, 6868-6882.

Spremulli, L.L. (2007). Large-scale isolation of mitochondrial ribosomes from mammalian tissues. Methods Mol. Biol. 372, 265-275.

Stewart, J.B., Freyer, C., Elson, J.L., Wredenberg, A., Cansu, Z., Trifunovic, A., and Larsson, N.-G. (2008). Strong Purifying Selection in Transmission of Mammalian Mitochondrial DNA. PLoS Biol 6, e10.

Stiburek, L., Fornuskova, D., Wenchich, L., Pejznochova, M., Hansikova, H., and Zeman, J. (2007). Knockdown of Human Oxa1I Impairs the Biogenesis of F1Fo-ATP Synthase and NADH:Ubiquinone Oxidoreductase. Journal of Molecular Biology 374, 506-516.

Stojanovski, D., Bragoszewski, P., and Chacinska, A. (2012). The MIA pathway: a tight bond between protein transport and oxidative folding in mitochondria. Biochim. Biophys. Acta 1823, 1142-1150.

Stoldt, S., Wenzel, D., Kehrein, K., Riedel, D., Ott, M., and Jakobs, S. (2018). Spatial orchestration of mitochondrial translation and OXPHOS complex assembly. Nature Cell Biology 1-11.

Stroud, D.A., Surgenor, E.E., Formosa, L.E., Reljic, B., Frazier, A.E., Dibley, M.G., Osellame, L.D., Stait, T., Beilharz, T.H., Thorburn, D.R., et al. (2016). Accessory subunits are integral for assembly and function of human mitochondrial complex I. Nature 538, 123-126. 
Styczynski, M.P., Jensen, K.L., Rigoutsos, I., and Stephanopoulos, G. (2008). BLOSUM62 miscalculations improve search performance. Nature Biotechnology 26, 274-275.

Subramaniam, S., Earl, L.A., Falconieri, V., Milne, J.L., and Egelman, E.H. (2016). Resolution advances in cryo-EM enable application to drug discovery. Current Opinion in Structural Biology 41, 194-202.

Sugiana, C., Pagliarini, D.J., McKenzie, M., Kirby, D.M., Salemi, R., AbuAmero, K.K., Dahl, H.-H.M., Hutchison, W.M., Vascotto, K.A., Smith, S.M., et al. (2008). Mutation of C20orf7 Disrupts Complex I Assembly and Causes Lethal Neonatal Mitochondrial Disease. The American Journal of Human Genetics 83, 468-478.

Swenson, S., Cannon, A., Harris, N.J., Taylor, N.G., Fox, J.L., and Khalimonchuk, O. (2016). Analysis of Oligomerization Properties of Heme a Synthase Provides Insights into Its Function in Eukaryotes. J. Biol. Chem. 291, 10411-10425.

Tait, S.W.G., and Green, D.R. (2012). Mitochondria and cell signalling. Journal of Cell Science 125, 807-815.

Takemoto, C., Spremulli, L.L., Benkowski, L.A., Ueda, T., Yokogawa, T., and Watanabe, K. (2009). Unconventional decoding of the AUA codon as methionine by mitochondrial tRNA Met with the anticodon $\mathrm{f} 5 \mathrm{CAU}$ as revealed with a mitochondrial in vitro translation system. Nucleic Acids Research 37, 1616-1627.

Taylor, N.G., Swenson, S., Harris, N.J., Germany, E.M., Fox, J.L., and Khalimonchuk, O. (2017). The Assembly Factor Pet117 Couples Heme a Synthase Activity to Cytochrome Oxidase Assembly. J. Biol. Chem. 292, 1815-1825.

Temperley, R.J., Wydro, M., Lightowlers, R.N., and Chrzanowska-Lightowlers, Z.M. (2010). Human mitochondrial mRNAs-like members of all families, similar but different. BBA - Bioenergetics 1797, 1081-1085.

Thompson, A.K., Smith, D., Gray, J., Carr, H.S., Liu, A., Winge, D.R., and Hosler, J.P. (2010). Mutagenic Analysis of Cox11 of Rhodobacter sphaeroides: Insights into the Assembly of $\mathrm{Cu}_{B}$ of Cytochrome cOxidase. Biochemistry 49, 5651-5661.

Timón-Gómez, A., Nývltová, E., Abriata, L.A., Vila, A.J., Hosler, J., and Barrientos, A. (2018). Mitochondrial cytochrome $c$ oxidase biogenesis: Recent developments. Seminars in Cell and Developmental Biology 76, 163-178.

Tsuboi, M., Morita, H., Nozaki, Y., Akama, K., Ueda, T., Ito, K., Nierhaus, K.H., and Takeuchi, N. (2009). EF-G2mt Is an Exclusive Recycling Factor in Mammalian Mitochondrial Protein Synthesis. Molecular Cell 35, 502-510.

User, N. (2001). Structural basis for the interactionof antibiotics with the peptidyltransferase centre in eubacteria. 1-8. 
van der Bliek, A.M., Sedensky, M.M., and Morgan, P.G. (2017). Cell Biology of the Mitochondrion. Genetics 207, 843-871.

van der Laan, M., Meinecke, M., Dudek, J., Hutu, D.P., Lind, M., Perschil, I., Guiard, B., Wagner, R., Pfanner, N., and Rehling, P. (2007). Motor-free mitochondrial presequence translocase drives membrane integration of preproteins. Nature Cell Biology 9, 1152-1159.

van der Laan, M., Wiedemann, N., Mick, D.U., Guiard, B., Rehling, P., and Pfanner, N. (2006). A Role for Tim21 in Membrane-Potential-Dependent Preprotein Sorting in Mitochondria. Current Biology 16, 2271-2276.

van der Sluis, E.O., Bauerschmitt, H., Becker, T., Mielke, T., Frauenfeld, J., Berninghausen, O., Neupert, W., Herrmann, J.M., and Beckmann, R. (2015). Parallel Structural Evolution of Mitochondrial Ribosomes and OXPHOS Complexes. Genome Biology and Evolution 7, 1235-1251.

van Dijk, A.D.J., Ciofi-Baffoni, S., Banci, L., Bertini, I., Boelens, R., and Bonvin, A.M.J.J. (2007). Modeling Protein-Protein Complexes Involved in the Cytochrome cOxidase Copper-Delivery Pathway. J. Proteome Res. 6, 15301539.

Vartak, R.S., Semwal, M.K., and Bai, Y. (2014). An update on complex I assembly: the assembly of players. J Bioenerg Biomembr 46, 323-328.

Vidoni, S., Harbour, M.E., Guerrero-Castillo, S., Signes, A., Ding, S., Fearnley, I.M., Taylor, R.W., Tiranti, V., Arnold, S., Fernandez-Vizarra, E., et al. (2017). MR-1S Interacts with PET100 and PET117 in Module- Based Assembly of Human Cytochrome c Oxidase. Cell Rep 18, 1727-1738.

Vilardo, E., Nachbagauer, C., Buzet, A., Taschner, A., Holzmann, J., and Rossmanith, W. (2012). A subcomplex of human mitochondrial RNase $P$ is a bifunctional methyltransferase-extensive moonlighting in mitochondrial tRNA biogenesis. Nucleic Acids Research 40, 11583-11593.

Vinothkumar, K.R., Zhu, J., and Hirst, J. (2014). Architecture of mammalian respiratory complex I. Nature 515, 80-84.

Vogel, R.O., Dieteren, C.E.J., van den Heuvel, L.P.W.J., Willems, P.H.G.M., Smeitink, J.A.M., Koopman, W.J.H., and Nijtmans, L.G.J. (2007a). Identification of mitochondrial complex I assembly intermediates by tracing tagged NDUFS3 demonstrates the entry point of mitochondrial subunits. Journal of Biological Chemistry 282, 7582-7590.

Vogel, R.O., Janssen, R.J.R.J., Ugalde, C., Grovenstein, M., Huijbens, R.J., Visch, H.-J., van den Heuvel, L.P., Willems, P.H., Zeviani, M., Smeitink, J.A.M., et al. (2005). Human mitochondrial complex I assembly is mediated by NDUFAF1. FEBS Journal 272, 5317-5326.

Vogel, R.O., Janssen, R.J.R.J., van den Brand, M.A.M., Dieteren, C.E.J., Verkaart, S., Koopman, W.J.H., Willems, P.H.G.M., Pluk, W., van den Heuvel, L.P.W.J., Smeitink, J.A.M., et al. (2007b). Cytosolic signaling protein Ecsit 
also localizes to mitochondria where it interacts with chaperone NDUFAF1 and functions in complex I assembly. Genes \& Development 21, 615-624.

Vogel, R.O., Smeitink, J.A.M., and Nijtmans, L.G.J. (2007c). Human mitochondrial complex I assembly: A dynamic and versatile process. Biochimica Et Biophysica Acta (BBA) - Bioenergetics 1767, 1215-1227.

Vogel, R.O., van den Brand, M.A.M., Rodenburg, R.J., van den Heuvel, L.P.W.J., Tsuneoka, M., Smeitink, J.A.M., and Nijtmans, L.G.J. (2007d). Investigation of the complex I assembly chaperones B17.2L and NDUFAF1 in a cohort of $\mathrm{Cl}$ deficient patients. Molecular Genetics and Metabolism 91, 176182.

Vögtle, F.N., Wortelkamp, S., Zahedi, R.P., Becker, D., Leidhold, C., Gevaert, K., Kellermann, J., Voos, W., Sickmann, A., Pfanner, N., et al. (2009). Global analysis of the mitochondrial $\mathrm{N}$-proteome identifies a processing peptidase critical for protein stability. Cell 139, 428-439.

Voorhees, R.M., Weixlbaumer, A., Loakes, D., Kelley, A.C., and Ramakrishnan, V. (2009). Insights into substrate stabilization from snapshots of the peptidyl transferase center of the intact 70 S ribosome. Nat Struct Mol Biol 16, 528-533.

Wagner, K., Rehling, P., Szklarz, L.K.S., Taylor, R.D., Pfanner, N., and van der Laan, M. (2009). Mitochondrial F1Fo-ATP Synthase: The Small Subunits e and $g$ Associate with Monomeric Complexes to Trigger Dimerization. Journal of Molecular Biology 392, 855-861.

Wang, D.D.-H., Guo, X.E., Modrek, A.S., Chen, C.-F., Chen, P.-L., and Lee, W.-H. (2014). Helicase SUV3, polynucleotide phosphorylase, and mitochondrial polyadenylation polymerase form a transient complex to modulate mitochondrial mRNA polyadenylated tail lengths in response to energetic changes. J. Biol. Chem. 289, 16727-16735.

Weidberg, H., and Amon, A. (2018). MitoCPR-A surveillance pathway that protects mitochondria in response to protein import stress. Science 360, eaan4146.

Weraarpachai, W., Antonicka, H., Sasarman, F., Seeger, J., Schrank, B., Kolesar, J.E., Lochmüller, H., Chevrette, M., Kaufman, B.A., Horvath, R., et al. (2009). Mutation in TACO1, encoding a translational activator of COX I, results in cytochrome $c$ oxidase deficiency and late-onset Leigh syndrome. Nature Genetics 41, 833-837.

Weraarpachai, W., Sasarman, F., Nishimura, T., Antonicka, H., Auré, K., Rötig, A., Lombès, A., and Shoubridge, E.A. (2012). Mutations in C12orf62, a factor that couples COX I synthesis with cytochrome $c$ oxidase assembly, cause fatal neonatal lactic acidosis. Am. J. Hum. Genet. 90, 142-151.

Wiedemann, N., and Pfanner, N. (2017). Mitochondrial Machineries for Protein Import and Assembly. Annu. Rev. Biochem. 86, 685-714. 
Wiedemann, N., van der Laan, M., Hutu, D.P., Rehling, P., and Pfanner, N. (2007). Sorting switch of mitochondrial presequence translocase involves coupling of motor module to respiratory chain. The Journal of Cell Biology $179,1115-1122$.

Wielburski, A., and Nelson, B.D. (1983). Evidence for the sequential assembly of cytochrome oxidase subunits in rat liver mitochondria. Biochem. J. 212, 829-834.

Wikström, M. (1984). Two protons are pumped from the mitochondrial matrix per electron transferred between NADH and ubiquinone. FEBS Letters 169, 300-304.

Wikström, M., and Sharma, V. (2018). Proton pumping by cytochrome $c$ oxidase - A 40 year anniversary. BBA - Bioenergetics 1-0.

Wikström, M., Krab, K., and Sharma, V. (2017). Oxygen Activation and Energy Conservation by Cytochrome cOxidase. Chem. Rev. 118, 2469-2490.

Winklhofer, K.F., and Haass, C. (2010). Mitochondrial dysfunction in Parkinson's disease. BBA - Molecular Basis of Disease 1802, 29-44.

Wirth, C., Brandt, U., Hunte, C., and Zickermann, V. (2016). Structure and function of mitochondrial complex I. BBA - Bioenergetics 1857, 902-914.

Yaguchi, T., Aida, S., Kaul, S.C., and Wadhwa, R. (2007). Involvement of Mortalin in Cellular Senescence from the Perspective of its Mitochondrial Import, Chaperone, and Oxidative Stress Management Functions. Annals of the New York Academy of Sciences 1100, 306-311.

Yakubovskaya, E., Guja, K.E., Eng, E.T., Choi, W.S., Mejia, E., Beglov, D., Lukin, M., Kozakov, D., and Garcia-Diaz, M. (2014). Organization of the human mitochondrial transcription initiation complex. Nucleic Acids Research 42, 4100-4112.

Yakubovskaya, E., Mejia, E., Byrnes, J., Hambardjieva, E., and Garcia-Diaz, M. (2010). Helix Unwinding and Base Flipping Enable Human MTERF1 to Terminate Mitochondrial Transcription. Cell 141, 982-993.

Yamano, K., Yatsukawa, Y.-I., Esaki, M., Hobbs, A.E.A., Jensen, R.E., and Endo, T. (2008). Tom20 and Tom22 share the common signal recognition pathway in mitochondrial protein import. Journal of Biological Chemistry 283, 3799-3807.

Yang, D., Oyaizu, Y., Oyaizu, H., Olsen, G.J., and Woese, C.R. (1985). Mitochondrial origins. Proc Natl Acad Sci USA 82, 4443-4447.

Zara, V., Ferramosca, A., Robitaille-Foucher, P., Palmieri, F., and Young, J.C. (2009). Mitochondrial carrier protein biogenesis: role of the chaperones Hsc70 and Hsp90. Biochem. J. 419, 369-375.

Zhang, X., Gao, X., Coots, R.A., Conn, C.S., Liu, B., and Qian, S.-B. (2015). 
Translational control of the cytosolic stress response by mitochondrial ribosomal protein L18. Nat Struct Mol Biol 22, 404-410.

Zurita Rendon, O., and Shoubridge, E.A. (2012). Early complex I assembly defects result in rapid turnover of the ND1 subunit. Human Molecular Genetics 21, 3815-3824.

Zurita Rendón, O., Silva Neiva, L., Sasarman, F., and Shoubridge, E.A. (2014). The arginine methyltransferase NDUFAF7 is essential for complex I assembly and early vertebrate embryogenesis. Human Molecular Genetics 23, 5159-5170.

Žárský, V., and Doležal, P. (2016). Evolution of the Tim17 protein family. Biol. Direct 1-13. 


\section{Acknowledgements}

First and foremost, I would like to thank Prof. Dr. Peter Rehling for the opportunity to do my $\mathrm{PhD}$ in his lab and for giving me the chance to work on this interesting project. I would like to express my gratitude for his full support, patience and guidance throughout these past four years.

I would like to thank my thesis committee members Prof. Dr. Markus Bohnsack and Prof. Dr. Henning Urlaub for their constructive criticisms, their suggestions and helpful discussions during the meetings.

I am especially grateful to Dr. Sven Dennerlein for his supervision, inspiring discussions and guidance for my projects and all his help both in lab and private issues.

I would like to thankProf. Dr. Holger Stark and Dr. Prakash Dube for analysis of my sample via electron microscopy, was well as for useful advices for my project.I also have to thank Dr. Bernhard Schmidt and Olaf Bernhard as well asProf. Dr. Henning Urlaub and Andreas Lindenfor the mass spectrometry analysis of my samples.

I would like to thank also my current and former lab members for creating a stimulating environment. I am especially grateful to my wonderful friend Dr. Isotta Lorenzi, who never stressed me once during all these years.

Thanks to Dr. Abhishek Aich and Dr. Isotta Lorenzi for useful advices in daily lab work and answering all my questions and Frank Richter and Dr. Alexander Schendzielorz for help with mitochondrial import. I also want to thank Dr. Ricarda Richter-Dennerlein, Elisa Kolander and Ridhima Gomkale for insightful discussions and advices on handling the mitochondrial ribosome and protein purification.

I would like to thank Dr. Sven Dennerlein,Dr. Abhishek Aich and especially Dr. Sylvie Callegari for proofreading my thesis and Dr. David Pacheu Grau to provide me information of mitochondrial complex I.

I would like to thank GGNB and the Molecular Biology of cells programm for their commitment to higher education and Petra Engelmann for administrative support.

A special thank to all my friends for joyful moments in past and future. 
Finally I am grateful to my family who has always supported me and helped me grow and become the person I am today. 